\title{
2021 ACMT Annual Scientific Meeting Abstracts-Virtual
}

\begin{abstract}
These are the abstracts of the 2021 American College of Medical Toxicology (ACMT) Annual Scientific Meeting. Included here are 178 abstracts that will be presented in April 2021, including research studies from around the globe and the ToxIC collaboration, clinically significant case reports describing toxicologic phenomena, and encore research presentations from other scientific meetings.
\end{abstract}

Keywords: Abstracts - Annual Scientific Meeting - Toxicology Investigators Consortium - Medical Toxicology Foundation

Correspondence: American College of Medical Toxicology (ACMT) 10645 N. Tatum Blvd Phoenix, AZ; info@acmt.net

Introduction: The American College of Medical Toxicology (ACMT) received 188 eligible research abstracts for consideration for presentation at the 2021 Annual Scientific Meeting (ASM), including 85 research studies and 103 case reports. Each abstract was reviewed in a blinded fashion by at least three Research Committee members. Each abstract was independently scored based on the clinical question, data source, analytic method, results/conclusion, and clarity of presentation. A total of 178 abstracts were accepted. This work would not be possible without the hard work and diligence of our abstract reviewers: Justin Arnold, Katie Boyle, Jeffrey Brent, Keith Burkhart, Jennifer Carey, Stephanie Carreiro, James Chenowth, Neeraj Chhabra, Jon Cole, Nick Connors, Fiona Garlich, Spender Greene, Rob Hendrickson, Michelle Hieger, David Juurlink, Louise Kao, Kenneth Katz, Russ Kerns, Andrew King, Kathy Kopec, JoAn Laes, Eric Lavonas, Michael Levine, David Liss, Kevin Maskell, William Meggs, Andrew Monte, Elissa Moore, Babak Mostafazadeh, Mark Mycyk, Mark Neavyn, Travis Olives, Patricia Rosen, Evan Schwarz, Samuel Stellpflug, Mark Su, Manoj Tyagi, Shawn Varney, Steven Walsh, Richard Wang, Sage Wiener, Brandon Wills, and Luke Yip. We would also like to thank the Fellows who participated in the inaugural review mentorship program: Vincent Calleo, Richard Chen, Joseph Kennedy, Jacob Lebin, and Christopher Meaden. Equally significant is the contribution of the ACMT staff (Adrienne Dunavin and Sandra Kirilovic) who led the process. Congratulations to all the contributors whose work will be presented in April.

We look forward to seeing you there.

Christine Murphy, MD, FACMT, Abstract Review Chair; Peter Chai, MD, FACMT, Abstract Review Co-Chair; Maryann Amirshahi, PharmD, MD, MPH, PhD, FACMT, Chair, ACMT Research Committee

\section{ACMT Annual Scientific Meeting Abstracts_-Virtual}

Day 1: Platforms, Abstracts 001-004
001. Gender-Based Production of N-Acetyl-P-Benzoquinone Imine Protein Adduct Formation With Therapeutic Acetaminophen Administration

Brandon J Sonn ${ }^{1}$, Kennon J Heard ${ }^{1,2}$, Susan M Heard ${ }^{2}$, Angelo $\mathrm{D}^{\prime}$ Alessandro ${ }^{1}$, Kate M Reynolds ${ }^{2}$, Richard C Dart ${ }^{2}$, Barry H Rumack $^{1,2}$, Andrew A Monte ${ }^{1,2}$

${ }^{1}$ University of Colorado Denver, Aurora, Colorado, USA. ${ }^{2}$ Rocky Mountain Poison and Drug Center, Denver, Colorado, USA

Background: Acetaminophen (APAP)-associated transaminase elevation, induced by N-acetyl-p-benzoquinone imine (NAPQI) protein adduction, remains a global concern. Distinct from known genetic, physiologic, and dosage associations dictating severity of hepatic injury, no known factors predict an absence of NAPQI protein adduct formation at therapeutic APAP dosing. Hypothesis: Gender-based physiologies are predictive of APAP-induced protein adduct formation at therapeutic doses.

Methods: This retrospective study is interrogating serum samples collected for a prior study investigating fluctuations of alanine aminotransferase (ALT) over time with 4 grams daily APAP dosing for $\geq 16$ days in subjects from Denver, Colorado. Subjects were grouped by NAPQI adduct formation $(n=184)$ vs no adducts $(n=20)$. Samples were run on ultra-high performance liquid chromatography mass spectrometry from days $0,7,16$, and 31. Significant metabolite expressions were identified using $t$-tests with false discovery rate correction (FDR), partial least squares discriminant, and ANOVA simultaneous comparison analyses. Demographic and clinical data were explored using $t$-tests with FDR (age, weight, BMI, ALT) and chi-square (gender, ethnicity, race) analyses.

Results: In pre-treatment samples, relative quantitation caprylic acid was expressed nine-fold higher and 6-carboxyhexanoate was expressed threefold lower in subjects who did not develop adducts. Lactate had greater expression in the no adducts group ( $p=\mathrm{NS}$ ). Using absolute quantitation, glutathione was expressed 2.6-fold greater among no adduct subjects. Odds of men developing NAPQI protein adducts at therapeutic APAP dosing are 5.91 times lower than women $(95 \% \mathrm{CI}=2.3-14.9 ; p=0.0001)$. Conclusion: Multiple metabolites were significantly expressed within the no adduct group with known greater expression in males, such as lactate. Additionally, metabolites were identified unique to adduct development independent of gender such as caprylic acid. At therapeutic APAP dosing, males are less likely to develop NAPQI protein adducts. Further research into lipid biosynthesis and metabolism may provide further insight into physiology associated with NAPQI production.

002. Platelet Mitochondrial Dysfunction in Acute Acetaminophen Toxicity and with 4-Methylpyrazole Treatment

Carolyn Fox, Michael Ekaney, Michael Runyon, Iain H McKillop, Christine Murphy

Atrium Health's Carolinas Medical Center, Charlotte, NC, USA 
Background: Clinical and experimental data suggest administration of 4methylpyrazole (4-MP) and N-acetylcysteine in severe acetaminophen (APAP) overdoses may improve morbidity and mortality. In the clinical setting, rapidly determining the degree of hepatic damage following APAP overdose remains challenging.

Hypothesis: Acetaminophen toxicity is ameliorated by 4-MP in an experimental model of APAP-toxicity in vivo, effects that are detectable by changes in circulating platelet mitochondrial function assessed by platelet-oxygen consumption (POC).

Methods: Male C57Bl/6 mice (8-12 weeks old) were randomized to receive APAP $(500$ or $300 \mathrm{mg} / \mathrm{kg}$ ) or vehicle $(0.9 \%[w / v]$ sodium chloride) intraperitoneally (ip) followed by either 4-MP treatment $(50 \mathrm{mg} / \mathrm{kg}$ ) or vehicle ip injection 90 minutes after APAP administration. Mice were euthanized 0 (control), 12, or 24 hours after APAP administration, and platelets from cardiac blood were isolated and counted. Using a closed system to measure POC, mitochondrial function was determined as a ratio of total oxygen consumption to platelet number. Differences between groups at each time point were evaluated by analysis of variance.
Results: Injection of $500 \mathrm{mg} / \mathrm{kg}$ APAP led to significantly changed POC within 12 hours versus both control and $300 \mathrm{mg} / \mathrm{kg}$ APAP $\left(-2.19 \pm 0.45 \times 10^{-7} \mu \mathrm{g} \mathrm{O} / \mathrm{min} /\right.$ platelet [500 $\mathrm{mg}$-APAP] versus $-5.52 \pm 1.73 \times 10^{-8} \mu \mathrm{g} \mathrm{O}_{2} / \mathrm{min} /$ platelet [Control] and $-7.22 \pm 0.70$ $\times 10^{-8} \mu \mathrm{g} \mathrm{O}_{2} / \mathrm{min} /$ platelet [300 mg-APAP] $P<0.05,500 \mathrm{mg}$-APAP versus Control and $300 \mathrm{mg}$-APAP), effects that were maintained at 24 hours. Administration of 4-MP did not significantly affect POC in control or $300 \mathrm{mg} / \mathrm{kg}$-APAP mice. Conversely, in mice receiving 500 $\mathrm{mg} / \mathrm{kg}-\mathrm{APAP}+4-\mathrm{MP}, \mathrm{POC}$ changed at both 12 hours $(-2.19 \pm 0.45 \times$ $10^{-7} \mathrm{\mu g} \mathrm{O}_{2} / \mathrm{min} /$ platelet [500 mg-APAP] versus $-1.10 \pm 0.15 \times 10^{-7}$ $\mu \mathrm{g} \mathrm{O}_{2} / \mathrm{min} / \mathrm{platelet}$ [500 mg-APAP+4-MP]) and 24 hours $(-2.22$ $\pm 0.49 \times 10^{-7} \mu \mathrm{g} \mathrm{O}_{2} / \mathrm{min} /$ platelet [500 $\mathrm{mg}$-APAP] versus $-0.81 \pm$ $0.12 \times 10^{-7} \mu \mathrm{g} \mathrm{O}_{2} / \mathrm{min} /$ platelet [500 mg-APAP+4-MP]). POC of mice treated with $500 \mathrm{mg} / \mathrm{kg}$-APAP+4-MP were not significantly different from time-matched vehicle and vehicle+4MP groups.

Conclusion: Platelet mitochondrial function can be used as an early biomarker of acute APAP toxicity, and 4-MP treatment may mitigate the harmful effects of APAP on platelet mitochondrial function.

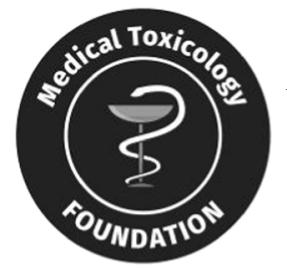

\section{This research was supported by a 2020 Medical Toxicology Foundation Innovative Research and Teaching Award.}

003. Late Hemotoxicity following North American Rattlesnake Envenomation Treated with Crotalidae Immune F(ab')2 (Equine) Antivenom (Anavip $\left.{ }^{\circledR}\right)$

Meghan B Spyres ${ }^{1,2}$, Gabriel Padilla ${ }^{3}$, Richard D Gerkin ${ }^{1,2}$, AnneMichelle Ruha ${ }^{1,2}$, On Behalf of the ToxIC Investigators Consortium (ToxIC)

${ }^{1}$ Banner University Medical Center Phoenix, Phoenix, AZ, USA. ${ }^{2}$ University of Arizona College of Medicine Phoenix, Phoenix, AZ, USA. ${ }^{3}$ University of Southern California Keck Medical School, Los Angeles, CA, USA

Background: Late hemotoxicity is common following rattlesnake envenomation treated with $\mathrm{Fab}$ antivenom $(\mathrm{CroFab} \otimes)$. Clinical trials showed Anavip ${ }^{\circledR}$ to be superior to $\mathrm{Crofab}{ }^{\circledR}$ in preventing late hemotoxicity, but this effect has not been demonstrated in broader populations.

Hypothesis: Patients with rattlesnake envenomation treated with Anavip ${ }^{\circledR}$ will have lower incidence of late hemotoxicity than those treated with Crofab®.

Methods: This is a multicenter analysis of prospectively collected data from patients with snakebite reported to the ToxIC NASBR spanning 2019. Inclusion criteria included administration of anti- venom for rattlesnake envenomation. Cases were excluded for incomplete data and if less than one set of follow-up laboratory values was obtained. Data collected included demographics, envenomation characteristics, laboratory values, and treatment administered. Statistics: $t$-test and Fisher's exact test. Hemotoxicity was defined as platelets $<120 \mathrm{~K} / \mathrm{mm}^{3}$ or fibrinogen $<170 \mathrm{mg} / \mathrm{dL}$; hemotoxicity was considered late on follow-up if values met threshold and were decreased from discharge value.

Results: One hundred thirteen rattlesnake bites receiving antivenom were reported to the NASBR in 2019, 111 were included. Twenty-seven received Anavip ${ }^{\circledR}$ alone, 50 received $\mathrm{Crofab}{ }^{\circledR}$ alone, and 34 received both antivenoms. Baseline patient and envenomation characteristics were similar between the groups. For single antivenom cases, Anavip ${ }^{\circledR}$ cases received more vials than Crofab ${ }^{\circledR}$ cases $(17$ vs. $11 ; p<0.001)$, but discrete dosing occurrences were similar ( 2.6 vs. 2.9 events; $p=0.11$ ). Late hemotoxicity occurred in 13 cases in the Crofab ${ }^{\circledR}$ group $(35.1 \%)$ and in 0 cases in the Anavip ${ }^{\circledR}$ group $(0.0 \%, 95 \%$ CI $0.00-0.16 ; p=0.001) ; 2$ (5.4\%) Crofab® cases required readmission and retreatment; no Anavip ${ }^{\circledR}$ cases were readmitted or retreated for hemotoxicity. One case (3.2\%) of late hemotoxicity was reported in patients receiving both Anavip ${ }^{\circledR}$ and Crofab®. Conclusion: Late hemotoxicity was not detected in NASBR Registry patients treated with Anavip ${ }^{\circledR}$ alone after rattlesnake envenomation; Crofab®-associated late hemotoxicity was similar to previous years.

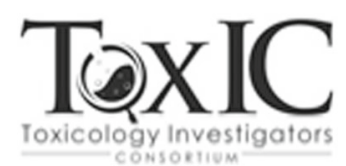

This research was performed in collaboration with the ACMT Toxicology Investigators Consortium. 
004. Linkage to Treatment and Tele-buprenorphine Post-emergency Department (ED) Opioid Overdose

Elizabeth A Samuels ${ }^{1}$, Michelle McKenzie ${ }^{2}$, Lee Ann Jordison Keeler ${ }^{3}$, Julia Uber $^{3}$, Neha Reddy ${ }^{1}$, Rachel S Wightman ${ }^{1}$

${ }^{I}$ The Warren Alpert Medical School of Brown University, Providence, RI, USA. ${ }^{2}$ The Miriam Hospital, Providence, RI, USA. ${ }^{3}$ Brown Emergency Medicine, Providence, RI, USA

Background: During this period of social distancing and isolation, people with opioid use disorder (OUD) are at increased risk of overdose death due to opioid withdrawal, loss of tolerance, using alone, and resumed use among people in recovery. In response to the COVID-19 pandemic, the Substance Abuse Mental Health Services Administration, the US Drug Enforcement Administration, and the US Department of Health and Human Services has allowed for use of telehealth for buprenorphine induction without requiring an in-person evaluation or video interface.

Research Question: What is the feasibility and utility of a follow-up telephone call to patients post-ED visit for opioid overdose to assess interest and, if appropriate, to initiate buprenorphine for OUD via a real-time telehealth encounter?

Methods: Trained research assistants attempted to contact all patients with working phone numbers who presented to the ED for an opioid overdose during a six-month period. Patients who were reached over the phone were queried on interest to engage in a real-time telehealth encounter to initiate buprenorphine. Eligible and interested patients were connected with an $\mathrm{x}-$ waivered provider for a telephone only telehealth encounter and, if clinically appropriate, provided a prescription and instructions for buprenorphine unobserved initiation and linkage to maintenance treatment.

Results: Three hundred and sixty-one patients presented to the ED for opioid overdose during the study period. Research assistants were able to connect with $127(35 \%)$ patients on the phone (41 accepted community substance use resources; 86 refused information). Nineteen patients expressed interest to speak to a buprenorphine prescriber and of those patients 11 engaged in a real-time telehealth consultation. Of the 127 patients reached via phone, six (5\%) were initiated on buprenorphine.

Conclusion: A post-ED telephone call protocol is a promising opportunity to improve treatment engagement and access to buprenorphine for patients at high risk for opioid overdose and death.

\section{Day 1: Moderated Posters, Abstracts 005-014}

\section{Cardiovascular Adverse Drug Reactions (ADRs) Associated with 4-Aminoquinolone (4-AQ) Drug Therapy During the COVID- 19 Pandemic}

Clare Gunn ${ }^{1}$, Mark Kastner ${ }^{1}$, Ann-Jeannette Geib ${ }^{1}$, Christine Murphy ${ }^{1}$, Satish Misra ${ }^{1,2}$, William Kerns ${ }^{1}$, Kathryn T Kopec ${ }^{1}$

${ }^{1}$ Carolinas Medical Center, Atrium Health, Charlotte, NC, USA. ${ }^{2}$ Sanger

Heart and Vascular Institute, Atrium Health, Charlotte, NC, USA

Background: During the COVID-19 pandemic, the FDA granted 4aminoquinolone (4-AQ) drugs (chloroquine, hydroxychloroquine) emergency use approval (EUA) from 3/28/2020 to 6/15/2020. 4-AQ drugs have potential adverse effects including hypotension, dysrhythmias, and prolonged QTc. Their benefit-risk ratio as a treatment for COVID-19 is unknown, especially in conjunction with additional QTc-prolonging medications.

Hypothesis: We assessed the demographics, dose, duration, and cardiovascular adverse drug reactions (ADRs) of 4-AQ in the treatment of COVID-19 patients within our health system.
Methods: This IRB-approved retrospective chart review used a standardized data abstraction form and included patients greater than 18 years old who received 4-AQ therapy for confirmed COVID-19 from 3/1/2020 to $6 / 15 / 2020$. Patients were identified through pharmacy distribution records. Patients were excluded if they received 4-AQ for other indications. Descriptive analysis and chi-square were performed.

Results: A total of 154 COVID-19 patients received at least one hydroxychloroquine dose; none received chloroquine. Eighty-two $(53 \%)$ were male and median age was 60 years. One hundred and two patients $(66 \%)$ received additional QTc prolonging drugs. Ninety patients (58\%) completed the 10-dose course, while 64 patients (42\%) did not. There were $67 / 154$ patients $(44 \%)$ who experienced cardiovascular events: hypotension 62 (93\%), dysrhythmias 23 (34\%). Of these 67 patients, 57 (85\%) were co-prescribed a QTc prolonging medication and 19 $(33 \%)$ developed dysrhythmias. The proportion of patients with dysrhythmias was similar between 4-AQ alone and 4-AQ plus other QTc prolonging drugs (chi-square $p=\mathrm{NS}$ ). Among patients co-prescribed a QTc prolonging medication, 65 (64\%) of 102 patients completed therapy. In this subset, cardiovascular ADRs occurred in 37 patients $(57 \%)$, including hypotension in 36/37 (97\%) and dysrhythmias in 8/37 (22\%). Prolonged QTc resulted in 4-AQ cessation in 7/64 patients with incomplete treatment course.

Conclusion: Cardiovascular ADRs were prevalent in patients receiving 4-AQ drug therapy when used as COVID-19 treatment.

\section{Public Interest in COVID Remedies Measured by Google Queries}

Wells Brambl, Stephanie L Widmer, Josh Nogar Northwell Health, Queens, NY, USA

Background: During the Covid-19 pandemic, unproven remedies were disseminated in the popular news media, possibly inspiring some to seek out these treatments. Several instances of toxicity and exposures necessitating medical evaluation have been documented.

Methods: Keywords Everywhere plugin tool was installed on Google Chrome. A global API from Google was obtained. Keywords queried were: hydroxychloroquine, chloroquine, bleach, azithromycin, covid vaccine, dexamethasone and Covid-19. The keywords were queried for relative search volume, utilizing "Trend volume data in bulk". Relative search volumes were tabulated by week during the past year leading up to November 10, 2020. Weekly relative search volumes inputted into Graph Pad Prism 9 for correlation Pearson coefficient and using Covid19 as a control. 2-tail analysis with a 95\% interval was selected.

Results: Search volumes per week for chloroquine, hydroxychloroquine, bleach, covid vaccine, and azithromycin all correlated statistically significant with searches for Covid-19 $(p<0.01)$. Of these, bleach had the highest R-coefficient of 0.9414. Searches for dexamethasone and Regeneron had inverse, but not statistically significant, correlation with searches for Covid-19. $(p>0.05)$. Furthermore, chloroquine search volume per week peaked at 827,500 during March, but dropped to 12,000 . Azithromycin had the highest peak at 1,452,400 during March, but dropped to 276,000 . Bleach peaked at 743,900 in May, but slowly dropped to 305,000 . Interestingly, total searches for Covid-19 have decreased by approximately $87 \%$. Complete data and graphs to be included. Conclusion: Despite the worsening pandemic, keyword searches for popularized Covid-19 treatments are decreasing. Although this is a market analysis, it may serve as a surrogate for public interest, implying that the public is less likely to be exposed to these xenobiotics. Limitations to the study include that it is performed using a proprietary keyword data extractor, with relative values based on projected data. Actual Google keyword search volumes are only available internally. 
007. The Role of High-Dose Folic Acid in Methanol Toxicity.

Alexander Sidlak, Ryan Marino, Matthew P Scanlon, Joshua A Shulman University of Pittsburgh Medical Center, Pittsburgh, PA, USA

Background: Folic acid has been proposed as an adjunct to alcohol dehydrogenase inhibitors and hemodialysis (HD) in the treatment of methanol toxicity. Though data regarding its efficacy are lacking, the risks of folic acid supplementation are minimal and animal models have shown benefit in expediting the clearance of formate.

Hypothesis: Folic acid administration leads to fewer adverse effects and shorter duration of intoxication for patients poisoned with methanol.

Methods: This is a retrospective chart review from 2012 to 2018 of patients with methanol concentrations $>10 \mathrm{mg} / \mathrm{dL}$. Patients receiving scheduled dosing of $50 \mathrm{mg}$ of folic acid or leucovorin (FG) were compared to those either receiving no adjunctive therapy or sub-therapeutic dosing of folic acid (NFG). Patients not requiring hospitalization, those with alternative causes of acidosis, and those who died prior to therapy were excluded. The primary outcome measures were worsening metabolic acidosis, length of stay (LOS), and half-life of methanol.

Result: Out of 27 patients identified with a positive methanol level, 14 (nine treated with folic acid, five not treated) met inclusion criteria. Initial $\mathrm{pH}$ and methanol levels were similar between the FG and NFG groups (7.29 vs. 7.26 and 118 vs. $120 \mathrm{mg} / \mathrm{dL}$ ). No difference in LOS was detected (3.3 d [IQR: $1.9-7.2]$ v $3.0 \mathrm{~d}$ [2.8-3.9]). No patients had worsening metabolic acidosis during therapy. In patients treated without and with $\mathrm{HD}$, the median half-life of methanol in FG vs. NFG was similar (2748 m [range: 1428-2997] vs. $2368 \mathrm{~m}$ [1945-2791]; $216 \mathrm{~m}$ [159-879 m] vs. $191 \mathrm{~m}$ [186-506]).

Conclusion: Folic acid treatment provided no additional benefit to standard therapy for methanol toxicity. We cannot rule out the benefit of an initial dose of folic acid while formate levels are elevated.

008. Fomepizole as an Adjunctive Therapy for Acetaminophen Poisoning: Cases Reported to the Toxicology Investigators Consortium (ToxIC) Database 2015-2020
Ari B Filip ${ }^{1}$, Sarah E Berg ${ }^{2}$, Michael E Mullins ${ }^{1}$, Evan S Schwarz ${ }^{1}$, On Behalf of the ToxIC Investigators Consortium (ToxIC)

${ }^{1}$ Washington University School of Medicine, St. Louis, MO, USA. ${ }^{2}$ Barnes Jewish Hospital, St. Louis, MO, USA ${ }^{3}$

Background: Fomepizole inhibits the formation of toxic acetaminophen (APAP) metabolites and may prevent or reverse mitochondrial toxicity. Given these mechanisms, it may be beneficial in select patients with toxicity from APAP.

Research Question: How have medical toxicologists used fomepizole in the treatment of APAP toxicity over the previous five years?

Methods: This is a retrospective analysis of patients enrolled in the Toxicology Investigators Consortium (ToxIC) database from January 2015 to July 2020. We queried cases in which APAP was listed as an ingested agent and fomepizole was also administered as an antidote. We excluded cases in which APAP was not the primary agent, NAC was not administered, or fomepizole was explicitly administered for an indication other than for APAP toxicity (e.g., toxic alcohol exposure). Additionally, we sent a survey to each ToxIC site that administered fomepizole for APAP toxicity to better understand when, why, and how they are using it for this indication.

Results: A total of 38 cases of fomepizole administration following an APAP ingestion were reported, with 25 cases meeting our inclusion criteria. There were one to four cases per year between 2015 and 2019 and eight cases in 2020. Seventeen of $25(68 \%)$ of cases were for known acute ingestion. Eighteen of $25(72 \%)$ of patients developed hepatotoxicity (AST $>1000$ ) and 10 of 25 (40\%) developed coagulopathy (PT $>15)$. This was an ill patient population, with 18 of $25(72 \%)$ developing metabolic acidosis $(\mathrm{pH}<7.20), 12$ of $25(48 \%)$ requiring intubation, 9 of 25 $(36 \%)$ receiving vasopressors, and 6 of 25 (24\%) requiring continuous renal replacement therapy. Overall, mortality was $24 \%$.

Conclusion: Fomepizole is increasing in frequency in a small subset of critically ill and acutely APAP-poisoned patients.

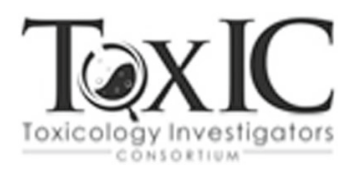

\section{This research was performed in collaboration with the ACMT Toxicology Investigators Consortium.}

\section{Acute Cariprazine Overdose in Adults: a 5-Year Retrospective Review from the California Poison Control System}

Paul F Ehlers ${ }^{1}$, Jacob A Lebin ${ }^{1,2}$, Anita K Mudan ${ }^{1,2}$, Daniel J Repplinger, ${ }^{1,2}$

${ }^{1}$ University of California, San Francisco, San Francisco, CA, USA.

${ }^{2}$ California Poison Control System, San Francisco, California, USA

Background: Cariprazine is a third-generation antipsychotic, first FDA approved in 2015 for treatment of schizophrenia and bipolar 1 disorder. It has a novel mechanism, acting as a $D_{3}$-preferring, $D_{3} / D_{2}$ partial receptor agonist. There are no published reports on the clinical effects of cariprazine after acute overdose. Therefore, we sought to characterize cariprazine exposures reported to the California Poison Control System (CPCS).

Methods: We performed a 5-year retrospective review of acute cariprazine exposures in adult patients reported to the California Poison Control System from 2015 to 2020. Patient characteristics, including demographics and ingestion type, as well as clinical course (symptoms, treatment, outcomes) were extracted from the CPCS database. Descriptive statistics were performed on the abstracted data, and categorical variables were compared by Fisher exact test.

Results: A total of 56 cases of cariprazine exposure were identified. The majority of cases were amongst women $(31 / 56,55 \%)$, intentional $(39 / 56$, $70 \%)$, and in combination with other ingestants $(41 / 56,73 \%)$. The median age was 32 years (range 19-82 years). Among single substance ingestions, exposure resulted in predominantly minor $(9 / 15,60 \%)$ or no $(5 / 15$, $33 \%$ ) effects. Among polysubstance ingestions, exposures resulted in major effects in five cases $(5 / 41,12 \%)$ or moderate effects in 10 cases $(10 / 41,24 \%)$, and effects were more commonly major or moderate in the polysubstance group than in in cariprazine single ingestions ( $p=0.005$ by Fisher exact test). There were no deaths in either cohort. Amongst all exposures, clinical manifestations were most commonly drowsiness (21\%), tachycardia (20\%), and dizziness (18\%). In patients with single substance exposure, there were no reports of seizure, QTc or QRS prolongation, respiratory depression requiring intubation, or hypotension 
requiring vasopressors. No specific therapy was administered in $73 \%$ of single substance ingestions.

Conclusion: Single substance cases of cariprazine exposure were generally self-limited and required no specific therapy.

\section{Perceptions on the Opioid Epidemic: a Qualitative Study}

Ariana McFarland, Brittany P Chapman, Rachael Davis-Martin, Kavita M Babu, Stephanie P Carreiro

University of Massachusetts Medical School, Worcester, MA, USA

Background: Interactions between patients with OUD and their healthcare providers can be challenging, particularly in emergency care settings. Negative healthcare experiences by OUD patients has been associated with worse clinical outcomes. Perceptual differences between patients and providers may provide clues to inform solutions.

Hypothesis: Patients with OUD and emergency providers will demonstrate discordant themes in perceptions on interactions with one another and OUD treatment approaches.

Methods: This was a qualitative study of patients and emergency providers (including paramedics, registered nurses, and physicians). Providers from a tertiary care academic medical center completed an online survey regarding perceptions on (1) interactions with patients with OUD, (2) medications for opioid use disorder (MOUD), and (3) bystander naloxone. Patients with OUD and a subset of providers completed semi-structured interviews covering the same topics. Interviews were conducted until thematic saturation was reached. Thematic analysis was conducted using a deductive coding structure from interview questions, followed by inductive coding after transcript review.

Results: Providers $(N=65)$ and patients $(N=22)$ identified as predominantly White, and approximately half were male. Emergent themes surrounding difficult interactions and perceptions on bystander naloxone differed between providers and patients, while themes surrounding MOUD use were similar. Regarding difficult interactions, providers focused on patient combativeness and inability to adequately treat patents' pain, while patients focused on feeling disrespected and lower priority than other patients. Regarding naloxone, providers did not perceive increased use of opioids due to availability but thought it could be a "safety net." Patients generally reported a strong aversion to receiving naloxone and denied personally using more opioids solely because naloxone was available. Both groups were similarly split on MOUD, with approximately half describing MOUD as a positive option for recovery, and half viewing MOUD as substituting "one addiction for another."

Conclusion: Emergency healthcare providers and patients with OUD identified perceptions that influence care. With further exploration, these can be used to inform interventions, decrease stigma, and improve healthcare quality for individuals with OUD.

011. Gender Differences in Nonmedical Use of Prescription Opioids, Tranquilizers and Stimulant Medications Using Machine Learning and Social Media

Yuan-Chi Yang $^{1}$, Mohammed Ali Al-Garadi ${ }^{1}$, Jennifer S. Love ${ }^{2}$, Jeanmarie Perrone ${ }^{3}$, Abeed Sarker ${ }^{1,4}$

${ }^{1}$ School of Medicine, Emory University, Atlanta, GA, USA. ${ }^{2}$ School of

Medicine, Oregon Health \& Science University, Portland, OR, USA.

${ }^{3}$ Perelman School of Medicine, University of Pennsylvania, Philadelphia,

PA, USA. ${ }^{4}$ Georgia Institute of Technology, Atlanta, GA, USA

Background: Medication misuse and overdose deaths are not evenly distributed across genders. For example, opioid overdose deaths have increased at a significantly greater rate in women compared to men since
2007. Understanding gender differences in medication misuse and overdose death may require integration of reports from federal agencies and social media as a complementary resource.

Hypothesis: Can we study gender differences in medication misuse from social media (Twitter) big data using machine learning?

Methods: This is an observational cohort study of self-reported nonmedical users of opioid, tranquilizer and stimulant prescription medications. Users were detected automatically via a supervised machine learning classifier. Users' publicly available data were aggregated and genders were inferred via a supervised machine learning classifier based on their public data (accuracy: 94.4\%). The gender distributions inferred from Twitter were compared with 2018 NSDUH reported gender distributions of nonmedical prescription medication usage and CDC Wonder database reported gender proportion of opioid overdose deaths.

Results: The Twitter cohort consisted of 176,683 users. Self-report among users found 38,299 misused opioids, 62,471 misused tranquilizers and 93,588 misused stimulants. Gender proportions for opioid misuse differed from NSDUH but were similar for opioid overdose death (Twitter: (male/female) $0.621 / 0.379$ vs. NSDUH: $0.518 / 0.482$ vs. CDC wonder: $0.685 / 0.315)$. Gender proportions of tranquilizer and stimulant misusers were similar for social media and NSDUH (Twitter: $0.499 / 0.501$ vs. NSDUH: $0.499 / 0.501$ for tranquilizers; $0.503 / 0.497$ vs. $0.551 / 0.449$ for stimulants).

Conclusion: We found associations between gender proportions of selfreported prescription medication misuse on Twitter and those of misuses or overdose death reported in government sources, supporting the usage of social media in understanding medication misuse. With its real-time platform, social media may serve as an important complementary data source for future evaluation. Our study can be extended to cover illicit substances.

012. Gender Differences in Emergency Department Substance Use Presentations and Substance Use Screens

Nikash Shankar ${ }^{1}$, Maryann Mazer-Amirshahi ${ }^{2}$, Kira Chandran ${ }^{1}$, Sameer Desale $^{3}$, Jeanmarie Perrone ${ }^{4}$, Mihriye Mete ${ }^{3}$

${ }^{I}$ Georgetown University School of Medicine, Washington, DC, USA. ${ }^{2}$ MedStar Washington Hospital Center, Washington, DC, USA. ${ }^{3}$ MedStar Health, Columbia, MD, USA. ${ }^{4}$ Perelman School of Medicine, Philadelphia, PA, USA

Background: Substance use disorder affects both men and women; however, there may be differences in the prevalence of substance use disorder and substances used based on gender.

Research Question: Are there gender differences in self-reported substance use when patients are screened during emergency department (ED) evaluation or with acute substance presentations?

Methods: Retrospective chart review using data from nine hospitals within a health system in the DC/Baltimore area of ED visits between 4/1/ 2019-6/30/2020. The health system utilizes a validated screening system for Screening, Brief Intervention, and Referral to Treatment (SBIRT) for ED patients who are clinically stable and willing to participate. We evaluated self-reported substance use during ED screening or acute intoxication/overdose presentations for men and women based on selfreported gender identity. Associations between type of substance used and gender were examined using chi-square tests.

Results: There were 319,130 screens performed during the study period. The study population was composed of Blacks $(60.2 \%)$, Whites $(32.1 \%)$, and other races $(7.7 \%)$. Women comprised $56.2 \%$ of screens. Problematic alcohol use (AUDIT C score $\geq 7$ ) or intoxication was more common among men $(7.2 \%)$ vs. women $(1.9 \%, p<0.001)$. Prescription opioid misuse was more prevalent among men vs. women $(1.6 \%$ vs. $0.6 \%, p<$ $0.001)$. Heroin use was reported by $2.4 \%$ of men vs. $1.0 \%$ of women $(p<$ $0.001)$. Men reported higher rates of cocaine use $(2.0 \%$ vs. $1.0 \% p<$ $0.001)$, prescription benzodiazepine use $(0.2 \%$ vs. $0.01 \%, p<0.001)$, 
and cannabis use compared to women $(7.5 \%$ vs. $4.4 \% p<0.001)$. Prevalence of use of other drugs was $10 \%$ for men and $5.8 \%$ for women $(p<0.001)$.

Conclusion: There were gender differences in self-reported substance use on ED screening and substance exposures, with men reporting significantly higher rates of use of all substances. While there may be limitations in reporting, this data can help inform ED interventions and referral to care

\section{Racial Differences in Emergency Department Substance Abuse} Presentations and Substance Abuse Screens

Nikash Shankar ${ }^{1}$, Maryann Mazer-Amirshahi ${ }^{2}$, Kira Chandran ${ }^{1}$, Sameer Desale $^{3}$, Lewis S Nelson ${ }^{4}$, Mihriye Mete ${ }^{5}$

${ }^{1}$ Georgetown University School of Medicine, Washington, DC, USA.

${ }^{2}$ MedStar Washington Hospital Center, Washington, DC, USA.

${ }^{3}$ MedStar Health, Columbia, MD, USA. ${ }^{4}$ Rutgers University, Newark,

NJ, USA. ${ }^{5}$ MedStar Washington Hospital Center, Columbia, MD, USA

Background: Substance use disorder occurs in individuals of all races; however, there may be differences in substances used based on race and other demographic and social factors.

Research Question: Are there racial differences in self-reported substance use when patients are screened during emergency department (ED) evaluation?

Methods: Retrospective chart review using data from nine hospitals within a health system in the DC/Baltimore area of ED visits between 4/1/ 2019 and 6/30/2020. The health system utilizes a validated screening system for Screening, Brief Intervention, and Referral to Treatment (SBIRT) for ED patients who are clinically stable and willing to complete screening. We evaluated self-reported substance use during ED screening based on self-identified race. Associations between type of substance used and race were examined using chi-square tests.

Results: There were 319,130 screens performed during the study period. The study population was composed of African Americans $(60.2 \%)$, Whites $(32.1 \%)$, and other races $(7.7 \%)$. Females comprised $56.2 \%$ of screens. Problematic alcohol use, defined as an AUDIT $\mathrm{C}$ score $\geq 7$, was more commonly reported by Whites vs. African Americans (5.2\% vs. 3.6\% of screens (other 5\%), $p<$ $0.001)$. Prescription opioid misuse was more commonly reported among White vs. African Americans (1.5\% vs $0.9 \%$ (other $0.4 \%$ ), $p<0.001)$, as was heroin ( $2.2 \%$ vs. $1.4 \%$ (other $0.3 \%), p<0.001$ ). Whites also reported higher rates of cocaine use $(1.7 \%$ vs. $1.4 \%$ (other $0.5 \%), p<0.001$ ) and prescription benzodiazepine abuse $(0.3 \%$ vs. $0.1 \%$ (other $0.1 \%$ ), $p<0.001$ ). African American patients reported higher rates of cannabis use compared to Whites $(7.3 \%$ vs. $3.7 \%$ (other $2.5 \%$ ), $p<0.001$ ).

Conclusion: There were racial differences in self-reported substance use on ED screening, with African Americans reporting higher rates of cannabis use and Whites with higher rates of use of all other substances. While there may be limitations in reporting, this data can help inform ED interventions and referral to care.

\section{Misuse of Pain Relievers by Age Group and Race Between 2015 and 2019: a National Analysis}

Christine Ramdin, Lewis Nelson

Rutgers New Jersey Medical School, Newark, NJ, USA

Background: There have been few recent reports comparing trends of misuse of pain relievers in those races and age groups that are traditionally as associated with opioid misuse. Due to the diversity of efforts in place to mitigate opioid misuse and manage addiction, we have undertaken this initiative to identify changes and trends.
Methods: We conducted a retrospective review of data collected from the National Survey of Drug Use and Health between 2015 and 2019 regarding pain reliever misuse among all identified races and age groups. We abstracted the percent of patients that reported misuse of pain relievers for Whites, African Americans, American Indian or Alaska Native, Asians (AA), Hispanics/Latinos, and Multiracial (two or more races) for all age groups. We reported means and used Pearson's correlation or Spearman's rho, to test trends for significance.

Results: Multiracial respondents had the largest rate of misuse compared to other races (mean: 6.08\%), particularly those aged 18-25 (mean: 9\% across years). A mean of $1.9 \%$ of Asians reported misuse and there were no changes within specific age groups. On average, 5.1\% of AA respondents reported misuse with no significant trends and $4.6 \%$ of White respondents reported misuse across years, with decreases for all age groups $(12+: p=0.003,12-17: p=0.002,18+: p=0.005,18-25: p=0.008,26+:$ $p=0.029)$. A mean of $3.9 \%$ of Black or African Americans and $4.3 \%$ of Hispanics and Latino respondents reported misuse between 2015 and 2019 and there were decreases for those $12+(p=0.009, p=0.0038)$, $12-17(p=0.018, p=0.02)$, and $18+(p=0.029, p=0.045)$.

Conclusion: There are decreases in reported opioid analgesic misuse for most age groups for Whites, Black/African Americans, and Hispanic/ Latinos. Multiracial respondents had the largest percent of current misusers. Future research should study strategies to sustain decreases and create targeted interventions to address nonresponding age and racial groups.

\section{Day 2: Platforms, Abstracts 015-018}

015. Succimer/CaNa $\mathbf{C}_{2}$ EDTA Combination Therapy for Nonencephalopathic Children with Blood Lead Concentration > 100mcg/dL: A Report of Five Cases

Kevin C Osterhoudt ${ }^{1}$, Reynaldo Hernandez ${ }^{2}$, Robert A Bassett ${ }^{1}$

${ }^{1}$ The Poison Control Center at Children's Hospital of Philadelphia, Philadelphia, PA, USA. ${ }^{2}$ Penn State-Hershey Medical Center, Hershey, PA, USA

Background: Varied chelation strategies for elevated blood concentrations (BLLs) exist; some recommend adding dimercaprol (BAL) for children with BLLs $>70 \mathrm{mcg} / \mathrm{dL}$ who are deemed "at risk for encephalopathy." As familiarity with the water-soluble BAL analog meso-2,3dimercaptosuccinic acid (succimer) has grown, and in light of challenges with BAL therapy, we hypothesize that combination therapy with succimer and edetate calcium disodium $\left(\mathrm{CaNa}_{2} \mathrm{EDTA}\right)$ may be appropriate for selected children with high BLLs.

Methods: Descriptive cohort of non-encephalopathic children with venous BLLs between 100 and $149 \mathrm{mcg} / \mathrm{dL}$, treated with combination therapy of succimer with $\mathrm{CaNa}_{2}$ EDTA, without BAL, between June 13, 2019, and November 12, 2020. Cases were obtained from records of our regional poison control center, and data was abstracted by a blinded investigator. This methodology with pre-existing data was exempt from Institutional Review Board review.

Results: Eight children with BLLs between 100 and $149 \mathrm{mcg} / \mathrm{dL}$ were identified; three treated with BAL (one who developed minor encephalopathic symptoms) were not included. Five were hospitalized (minimum five days) and treated with $\mathrm{CaNa}_{2}$ EDTA (five days) plus succimer (19 days). Age range was 14 months to 10 years; $4 / 5$ were male. The venous BLL at start of chelation ranged from 103 to $145 \mathrm{mcg} / \mathrm{dL}$. After five days of succimer plus EDTA, BLLs were reduced by a mean of $56 \%$ from the beginning value (range 46-64\%). For three subjects with complete data, at the end of 19 days of succimer, BLLs had declined by $86 \%, 75 \%$, and $63 \%$. None developed encephalopathic signs.

Conclusion: A previous cohort found comparable BLL reduction with succimer vs. BAL, combined with EDTA, in children with BLLs up to 90 $\mathrm{mcg} / \mathrm{d}$; we provide data on higher BLLs. Encephalopathy is a rare event; safety of succimer compared to BAL cannot be assessed by this study. 
016. A Look Back: E-Cigarette, or Vaping, Product Use Associated Lung Injury (EVALI) Before and After the COVID-19 Pandemic

Cherie Obilom ${ }^{1}$, Joshua McFalls ${ }^{1}$, Lindsey Claire Epperson ${ }^{1}$, Kerollos Shaker ${ }^{1}$, Jeffrey Brent ${ }^{2}$, Paul Wax ${ }^{1,3}$, Kim Aldy ${ }^{1,3}$, On Behalf of the ToxIC Investigators Consortium (ToxIC)

${ }^{1}$ University of Texas Southwestern Medical Center, Dallas, TX, USA.

${ }^{2}$ University of Colorado School of Medicine, Denver, CO, USA.

${ }^{3}$ American College of Medical Toxicology, Phoenix, AZ, USA

Background: Since the rise of the COVID-19 crisis in February 2020, attention has fallen away from the EVALI pandemic. However, EVALI cases continue to present to hospitals around the country.

Research Question: Has there been a change in demographics and vaping use patterns of EVALI before and after the COVID-19 pandemic? Methods: This is a retrospective review of vaping cases entered into the Toxicology Investigators Consortium (ToxIC) Registry from September 2019 to October 2020. Cases were analyzed in two groups, those reported before February 2020 and those reported after this date. Descriptive sta- tistics are used to compare demographics and vaping use patterns before and after the COVID-19 pandemic.

Results: A total of 74 vaping cases were identified, with 20 cases entered after the COVID-19 pandemic started. No cases entered AC reported COVID-19 positive status. The average age before COVID-19 (BC) was 22.5, and after COVID-19 (AC) was 19.4. The majority of patients BC were in the 19-65 age group (63\%), but AC most patients were 13-18 age group (80\%). Sex BC and AC comprised mostly of males $(66.7 \%$ and $80 \%$ respectively). THC only use $\mathrm{BC}$ and $\mathrm{AC}$ decreased from $46.3 \%$ vs $35 \%$, respectively, while nicotine only use nearly doubled from $5.6 \%$ vs. $10 \%$. Reported use of both THC and nicotine did not change significantly, increasing slightly from $33.3 \% \mathrm{BC}$ to $35 \% \mathrm{AC}$. Two deaths were reported after vaping exposure $\mathrm{BC}$, but no deaths have been reported to date AC.

Conclusion: EVALI has persisted through the COVID-19 pandemic, with the majority of vaping cases reported AC comprising of adolescents. This data suggests vaping has remained popular with adolescents despite the negative national attention and decreased opportunity for social interaction.

\section{ToxIC}

This research was performed in collaboration with the ACMT Toxicology Investigators Consortium.

017. Characteristic of Extracorporeal Membrane Oxygenation (ECMO) Use for Acute Pediatric Poisoning in the U.S.

Nicholas E Walter ${ }^{1}$, Matteo Di Nardo ${ }^{2}$, Danilo Alunnifegatelli ${ }^{3}$, Marco Marano $^{2}$, Reem Al Falasi ${ }^{1}$, Daniel L Herr ${ }^{4}$, Hong K Kim ${ }^{5}$

${ }^{1}$ Department of Emergency Medicine, University of Maryland Medical Center, Baltimore, MD, USA. ${ }^{2}$ Pediatric Intensive Care Unit, Bambino Gesu Children's Hospital, IRCCS, Rome, Rome, Italy. ${ }^{3}$ Department of Public Health and Infectious Disease, Sapienza University of Rome, Rome, Rome, Italy. ${ }^{4}$ Department of Internal Medicine, University of Maryland School of Medicine, Baltimore, MD, USA. ${ }^{5}$ Department of Emergency Medicine, University of Maryland School of Medicine, Baltimore, MD, USA

Background: Extracorporeal membrane oxygenation (ECMO) is not routinely utilized in the management of acutely poisoned pediatric patients.

Hypothesis: To describe the clinical and demographic characteristics of pediatric poisoning that required ECMO support.

Methods: We conducted a retrospective study using the Extracorporeal Life Support Organization's ECMO registry. Pediatric ECMO cases (age $<19$ years) were identified between January 1, 2003, to November 30, 2019, using International Classification of Diseases (ICD) codes for poisoning (ICD-9: 960-989 \& ICD-10: T36-T65). Each case's ICD codes were reviewed by study authors to identify acute poisoning related ECMO cases. Descriptive analysis was performed to assess demographic and clinical characteristics, including survival rate. Subgroup analysis was also performed, stratified by pre-ECMO vs. 24-hours post-ECMO, and survivors vs. non-survivors.

Results: One hundred sixty-nine cases were identified; 52 cases were excluded (39 non-poisoning and 13 non-acute poisoning cases), leaving 117 for final analysis. The median age was 14 years and $53.5 \%$ were female. Gas/fume was the most commonly involved exposure $(n=18)$ followed by hydrocarbon $(n=16)$. The intention of exposure was unknown in the majority of cases $(61.5 \%)$ followed by suicide $(20.5 \%)$. ECMO was initiated for pulmonary support in $46.2 \%$; 91 cases $(77.8 \%)$ received venoarterial-ECMO. The survival rate was $69.2 \%$ (venoarterial-
ECMO: $68.1 \%$ and venovenous-ECMO: $73.1 \%$ ). Comparison of pre and 24-hours post-ECMO showed improvement in $\mathrm{pH}$ (7.18 vs. $7.38 ; p<$ $0.001), \mathrm{HCO}_{3}(19.8$ vs. $25 \mathrm{mmol} / \mathrm{L} ; p<0.001)$, lactate ( 7.7 vs. 2.1 $\mathrm{mmol} / \mathrm{L} ; p<0.001)$, and mean blood pressure (58 vs. $72 \mathrm{mmHg} ; p<$ $0.001)$. Persistent acidosis and low systolic blood pressure $(<85 \mathrm{mmHg})$ were associated with in hospital mortality. There was no significant difference in adverse event rate or time from admission to ECMOcannulation between survivors and non-survivors.

Conclusion: ECMO was used in a wide range of pediatric poisoning in the US. Persistent metabolic acidosis and low systolic blood pressure were associated with mortality.

018. Reimbursement Parameters of Poisoned Patients Admitted for Observation Compared to Chest Pain Patients Admitted for Observation

Antonia Nemanich ${ }^{1}$, Theresa Kim ${ }^{2}$, Jerrold Leikin ${ }^{1,3}$

${ }^{1}$ Toxikon Consortium, Chicago, IL, USA. ${ }^{2}$ NorthShore University HealthSystem, Glenview, IL, USA. ${ }^{3}$ Illinois Poison Center, Chicago, IL, USA

Background: Poisoned patients who are well-appearing but at risk for potential decompensation often require admission for observation. Observation units often tailor to diagnoses such as chest pain. To date, little has been published on the financial reimbursement patterns of poisoned patients and whether this population would be a source for increased revenue.

Research Question: Is an observation unit for low-risk poisoned patients a source of significantly increased revenue?

Methods: The study period spanned October 2019-April 2020 at a network of four suburban hospitals in the Chicago area. A retrospective chart review of poisoned patients admitted for observation $\leq 3$ days was performed. Data on the number of patients admitted, length of stay, total costs, and collection rate was gathered. This was compared to patients during the same period observed for chest pain.

Results: Of the 52,668 total ED visits during the study period, 70 patients were admitted for observation with a diagnosis of poisoning. The 
majority (45, or $64.3 \%$ ) were observed for < 24 hours, 19 (27.1\%) were observed for one to two days, and only six patients $(8.6 \%)$ were admitted for two to three days. The gross collection rate (GCR) from these visits was $24.8 \%$ of the billed charges, averaging $\$ 3,728$ per patient. For comparison, data from chest pain admissions were reviewed. There were 444 patients admitted for observation of chest pain during the same period. Most (385, or $86.7 \%)$ were observed for $<24$ hours, $49(11.0 \%)$ were observed for one to two days, and only $10(2.3 \%)$ were admitted for two to three days. The GCR of chest pain admission was $25.0 \%$ of billed charges, averaging $\$ 4,521$ per patient.

Conclusion: GCR percentage for poisoned patients was comparable to that of observing chest pain patients $(\sim 25 \%)$, but the overall total collection rate was $\$ 793$ less per patient compared to chest pain admissions.

\section{Day 2: Moderated Posters, Abstracts 019-028}

\section{After Hours Poisoning and Overdose Presentations to Emergency Departments}

Pelayia H Soto ${ }^{1,2}$, Peter M Mullins ${ }^{3}$, Lewis S Nelson ${ }^{4}$, Maryann MazerAmirshahi $^{5}$

${ }^{1}$ National Capital Poison Center, Washington, DC, USA. ${ }^{2}$ George Washington University School of Medicine, Department of Emergency Medicine, Washington, DC, USA. ${ }^{3}$ Brigham and Women's Hospital, Department of Emergency Medicine, Boston, MA, USA. ${ }^{4}$ Rutgers University School of Medicine, Department of Emergency Medicine, Newark, NJ, USA. ${ }^{5}$ MedStar Washington Hospital Center, Department of Emergency Medicine, Washington, DC, USA

Background: Emergency departments (EDs) manage the majority of acute presentations for poisoning and overdose. Knowledge of when these patients present can help inform use of ED psychiatry and substance use resources.

Research Question: This study examines the role of EDs in managing visits for poisoning and overdose outside of regular business hours.

Methods: We analyzed the National Hospital Ambulatory Medical Care Survey (NHAMCS), an annual national survey of ED visits, from 2015 to 2017. Because NHAMCS is publicly accessible and deidentified, this study was not subject to IRB review. We examined all ED visits related to poisoning and overdose. Business hours were defined as 0800-1700 Monday-Friday, with all other times defined as after hours. We compared demographics, hospital factors, resource utilization, and care intensity across time of visit using Pearson chi-square tests.

Results: From 2015 to 2017, there were 1.4 million ED visits for poisoning/overdose during business hours and 4.2 million visits after hours $(25.2 \%$ vs. $74.8 \% ; p<0.001)$. For all ED visits, after hours visits accounted for $65 \%$ of total visits. There were no differences in age, gender, race, or insurance status based on the time of presentation. Medicaid was the most common insurance for poisoning/overdose visits regardless of time of presentation at $42.2 \%$. Emergency severity index was the same for both time groups. Rates of diagnostic imaging, laboratory testing, number of medications administered, and procedures performed were not different between time groups. Length of stay and rates of admission were also the same, regardless of time of presentation.

Conclusion: Patients presenting to the ED with acute poisoning or overdose were more common after hours than ED visits overall. While visit characteristics and resource utilization were similar, this data can be used to inform psychiatry, social work, and recovery staffing to improve access to care for this vulnerable population.

020. Evaluation of Toxicodendron Inhalation Exposures Reported to National Poison Data System (NPDS)
Faisal Syed Minhaj ${ }^{1}$, Chaya Lachman ${ }^{1}$, James B Leonard ${ }^{1}$, Joshua D King ${ }^{1,2}$, Bruce Anderson ${ }^{1}$

${ }^{1}$ University of Maryland School of Pharmacy, Baltimore, MD, USA.

${ }^{2}$ University of Maryland School of Medicine, Baltimore, MD, USA

Background: Found across most of North America, toxicodendron plants such as poison ivy, oak, and sumac are notorious for inducing contact dermatitis upon exposure to urushiol resins. While there is a plethora of information on dermal exposures, the only published literature on the toxicity of toxicodendron inhalations is a brief case report published in 1995.

Research Question: What symptoms are seen in patients with toxicodendron inhalation exposures, and what treatments are commonly required?

Methods: This is a retrospective observational study of all toxicodendron inhalations reported to NPDS from January 2000 to March 2020. Unrelated and confirmed non-exposure cases were excluded. The IRB determined this study to be exempt.

Results: A total of 1469 cases were included in the analysis with 519 having concurrent dermal exposures. Median age was 33 (IQR 24-45) with $870(59.2 \%)$ being male. Most patients were treated at home (1136, $77.3 \%)$ with $291(19.8 \%)$ referred or seen in a healthcare facility. The majority of cases were not followed $(1039,70.7 \%)$. A total of three $(0.2 \%)$ developed major effects, $82(5.6 \%)$ moderate, and $175(11.9 \%)$ minor effects. Of those seen in a HCF $(n=243)$, most were treated in the emergency room $(113,38.8 \%)$ with four $(1.6 \%)$ requiring admission, and two $(0.8 \%)$ admitted to a critical care unit. The most common symptoms were rash $(220,15.0 \%)$, throat irritation $(132,9.0 \%)$, pruritus $(126$, $8.6 \%)$, and coughing or choking $(87,6.0 \%)$. The most common pharmacologic treatments administered were antihistamines (135), steroids (90), bronchodilators (22), and antibiotics (14).

Conclusion: Most patients with inhalation of toxicodendrons can be successfully managed at home and have minor symptoms including rash, throat irritation, pruritus, and coughing or choking. Pharmacologic treatments commonly used for these patients include antihistamines, steroids, bronchodilators, and antibiotics.

021. Clinical Presentations, Treatments, and Outcomes of Exotic Snake Envenomations in the United States

Jack E Basse ${ }^{1}$, Spencer Greene ${ }^{2}$, Anne-Michelle Ruha ${ }^{3}$, Kevin Baumgartner ${ }^{1}$, Michael E Mullins ${ }^{1}$, Paul Wax ${ }^{4}$, Jeffrey Brent ${ }^{4}$, Sharan Campleman ${ }^{4}$, Evan S Schwarz ${ }^{1}$, On Behalf of the ToxIC Investigators Consortium (ToxIC)

${ }^{1}$ Washington University School of Medicine, St. Louis, MO, USA. ${ }^{2}$ University of Houston College of Medicine, Houston, TX, USA. ${ }^{3}$ Banner University Medical Center Phoenix, Phoenix, AZ, USA. ${ }^{4}$ American College of Medical Toxicology, Phoenix, AZ, USA

Background: Exotic snake envenomations in the United States are uncommon, and antivenoms for non-native snakebites are not typically available in hospital pharmacies.

Research Question: This study described the clinical presentations, treatments, and outcomes of exotic snake envenomation cases reported to the Toxicology Investigators Consortium's (ToxIC) North American Snakebite Registry (NASBR).

Methods: This is a descriptive study that reviewed all non-native envenomations reported to the NASBR from 2013 to 2019. Data abstracted included snake species, patient history, clinical signs, diagnostics, treatment (including antivenom usage), follow-up, and final outcome.

Results: Eighteen non-native snakebites were reviewed, resulting from encounters with eleven different species, eight of which belonged to the Viperidae family. The most common presenting symptoms were edema (17 patients), ecchymosis (10 patients), and necrosis (four patients). Systemic effects and hematologic abnormalities were less common. The 
most commonly employed hospital treatments were noninvasive, but four patients did require mechanical ventilation. Eleven patients were treated with antivenom. Four victims of encounters with snakes for whom antivenoms exist received no antivenom. No patients died as a result of an encounter with an exotic snake; two experienced digit mobility loss, one experienced hand mobility loss, and one experienced permanent tissue loss of a small area on their finger.
Conclusion: While limited, the results of this study suggest that victims of exotic snake encounters in the US frequently present with soft tissue effects, and less frequently with systemic or hematologic effects. Antivenom is often used, although several patients for which a specific antivenom was indicated did not receive any. Long-term sequelae of such encounters are rare and usually consist of local mobility deficits.

\section{ToxIC}

This research was performed in collaboration with the ACMT Toxicology Investigators Consortium.

\section{Characteristics and Trends of Coral Snake Bites Reported to NPDS from 2006 to 2018}

\author{
Stephen L Thornton ${ }^{1}$, Micheal Darracq ${ }^{2}$ \\ ${ }^{1}$ Kansas Poison Control Center, Kansas City, KS, USA. ${ }^{2}$ University of \\ California-San Francisco-Fresno Department of Emergency Medicine, \\ Fresno, CA, USA
}

Background: Bites from North American coral snakes have the potential to cause significant morbidity and mortality due to their neurotoxic venom. However, coral snakes are rare and geographically limited resulting in limited medical literature on their bites.

Research Question: We sought to describe the characteristics and trends of coral snake bites reported to the National Poison Data System (NPDS) from 2006 to 2018 .

Methods: This was a retrospective review of coral snake cases reported to NPDS between January 1, 2006, and December 31, 2018 , that were followed to a known outcome. All data in the NPDS was analyzed using IBM SPSS Statistics for Mac, Version 25.0 (Armonk, NY).

Results: 856 cases were identified: 677 were adult (average age 40.7 years) and 179 were pediatric cases (average age 12.7 years). $79 \%(n=533)$ of adult and $74 \%(n=179)$ of pediatric cases were male. Cases were reported in every month with the most in April ( $n$ $=123$ ). The highest number of cases were reported in 2006 and 2008 (both 78 cases) and the lowest in 2016 (53 cases). Florida had the single year most cases with 45 in 2006. Eighteen states reported coral snake bites with Florida $(n=416)$ and Texas $(n=$ $359)$ accounting for $91 \%$ of all cases. Nine non-endemic states reported coral snake bites. Minor outcomes occurred in $57 \%(n=384)$ of adult and $56 \%(n=100)$ of pediatric cases. $44 \%(n=300)$ of adult and $46 \%(n=83)$ of pediatric cases were admitted to an ICU. Mechanical ventilation was reported in 16 adult and one pediatric case. Thirty four percent of adult $(n=228)$ and 33\% $(n=60)$ of pediatric cases received antivenom. No deaths were reported.

Conclusion: Coral snake bites were most often associated with minor outcomes and did not receive antivenom. More than $40 \%$ were admitted to an ICU but mechanical ventilation was rare and no deaths were reported.

023. Characteristics and Trends of Cottonmouth Bites Reported to NPDS from 2006 to 2018

Stephen L Thornton ${ }^{1}$, Michael Darracq ${ }^{2}$

${ }^{1}$ Kansas Poison Control Center, Kansas City, KS, USA. ${ }^{2}$ University of California-San Francisco-Fresno, Fresno, CA, USA
Background: Bites from North American crotalids have the potential to cause significant morbidity and mortality. The cottonmouth (Agkistrodon piscivorus) is often described as an aggressive snake with potential to cause significant envenomation. However, robust published literature on cottonmouth bites is limited.

Research Question: We sought to describe the clinical characteristics and trends in cottonmouth bites reported to the National Poison Data System (NPDS) from 2006 to 2018.

Methods: This was a retrospective study of all cottonmouth bites reported to the NPDS between January 1, 2006, and December 31, 2018, that were followed to a known outcome. All data in the NPDS was analyzed using IBM SPSS Statistics for Mac, Version 25.0 (Armonk, NY).

Results: Two thousand four hundred and seventy one cases were identified of which 1958 were adult (average age 40.2 years) and 513 were pediatric cases (average age 12.5 years). $79 \%(n=1546)$ of adult and $76 \%$ $(n=388)$ of pediatric cases were male. Cases were reported all months with the most in July $(n=368)$. The highest number of cases was reported in 2013 (242) and the lowest in 2007 and 2008 (both with 146 cases). Texas had the single year most cases with 77 in 2018. 34 states and the District of Columbia reported cottonmouth bites with Florida $(n=723)$, Texas $(n=459)$, and Louisiana $(n=338)$ reporting the most overall cases. Several cottonmouth non-endemic states (Utah, California, Arizona) reported cottonmouth bites. Moderate or major outcomes were documented in $55 \%(n=1073)$ of adult and $57 \%(n=295)$ of pediatric cases. Thirty percent $(n=580)$ of adult and 27\% $(n=140)$ of pediatric cases were admitted to an ICU. Antivenom was given in $49 \%$ of adult $(n=959)$ and $54 \%$ (276) of pediatric cases. One adult death was reported.

Conclusion: Cottonmouth bites were often associated with significant medical outcomes but rare mortality. Antivenom therapy was given in approximately $50 \%$ of cases.

024. New Psychoactive Substances Identified in 2020 via the Drug Enforcement Administration's Toxicology Program

Jordan Trecki ${ }^{1}$, Roy R Gerona ${ }^{2}$

${ }^{1}$ Drug Enforcement Administration, Washington, DC, USA. ${ }^{2}$ University of California at San Francisco, San Francisco, CA, USA

Background: Since emerging as a public health threat in the late 2000s, new psychoactive substances (NPS), also referred to as synthetic drugs, have continued to evolve in an effort to avoid prosecution and drug testing. Abuse of synthetic cannabinoids (spice/K2), synthetic cathinones (bath salts), synthetic opioids, fentanyl-related substances, novel benzodiazepines, LSD-type hallucinogens and substances from other drug classes have been shown to cause serious adverse reactions including death to the user following their ingestion. Physicians are required to treat the 
symptoms of synthetic drug overdoses and in most cases do not know if or what synthetic drugs may have been responsible for the adverse effects. Hypothesis: Without advanced analysis via a commercial or academic toxicology laboratory, routine clinical toxicology screens will fail to detect NPS due to lack of standards and analytical equipment.

Methods: Via an awarded contract with the University of California at San Francisco, the Drug Enforcement Administration has actively sought leftover blood samples from overdose patients throughout the United States when a synthetic drug overdose was suspected. Upon receipt, samples are analyzed by means of liquid chromatography-quadrupole timeof-flight mass spectrometry. Results of the analysis are then transmitted to the originating physician within three weeks.

Results: Between January and September 2020, 42 cases were accepted from across the US. In 33 out of the 42 cases, the program identified synthetic drugs that the submitting facility was not able to detect. Substances detected included 5F-MDMB-PICA, 5F-ADB, and MMBFUBINACA (synthetic cannabinoids), 2,5-DMA [2,5-dimethoxy-amphetamine] (empathogen/entactogen), p-chlorophenylpiperazine [pcPP] (stimulant), acetyl fentanyl and beta-hydroxy fentanyl (fentanyl-related substances), 5-hydroxynordeschloroketamine and 2-fluoro deschloroketamine (dissociative anesthetics), flualprazolam (novel benzodiazepine), and mitragynine and 7-OH mitragynine (kratom alkaloids), amongst others.

Conclusion: As NPS/synthetic drugs continue to evolve, neither the user nor the treating physician can be sure of what was ingested without advanced toxicological testing.

\section{Fentanyl and Fentanyl Analogues in ED Patients with Suspected Heroin Overdose in the Pre-COVID Era}

Alex F Manini ${ }^{1,2}$, Alex J Krotulski ${ }^{3}$, Lisa Allen ${ }^{1}$, Jeffery Brent ${ }^{4}$, Valeria Samame $^{1}$, Kavey Vidal ${ }^{1}$, Paul Wax ${ }^{5}$, Barry Logan ${ }^{3,6}$

${ }^{1}$ Icahn School of Medicine at Mount Sinai, New York, NY, USA. ${ }^{2}$ Elmhurst Hospital Center, New York, NY, USA. ${ }^{3}$ Center for Forensic Science Research \& Education; Fredric Rieders Family Foundation, Willow Grove, PA, USA. ${ }^{4}$ University of Colorado, School of Medicine, Aurora, CO, USA. ${ }^{5}$ UT Southwestern Medical Center, Dallas, TX, USA. ${ }^{6}$ NMS Labs, Horsham, PA, USA

Background: There were $>81,000$ drug overdose deaths in the USA last year, the highest ever. Global drug commerce introduced synthetic opioid fentanyl analogues (fentalogs) into the drug supply in the Pre-COVID Era. Research Question: We describe prevalence of fentalog OD in ED patients with suspected heroin OD, and associations with treatment requirements.

Methods: This was an observational cohort in the pre-COVID five-year period (2015-2020) at two urban teaching hospitals. Adult (> 18 years) ED patients with suspected heroin OD were included if waste serum was available; exclusion criteria were prisoners, non-opioid $\mathrm{OD}$, and alternate diagnoses. De-identified data was abstracted form the medical record. Toxicological confirmation was performed via liquid chromatography/ quadrupole time-of-flight mass spectrometry in a panel of $>800$ drugs including novel substances. Treatment requirements were defined as (a) naloxone administration (total dose, infusion, repeat bolus), and (b) ED disposition (ICU, medicine, discharge).

Results: Of 94 patients, there were 14 fentalogs (14.9\%, CI 8.4-23.7), 57 fentanyl $(60.6 \%$, CI 50-70.6), 60 either fentanyl or fentalogs $(63.8 \%$, CI 53.2-73.5), 50 heroin (53.2\%, CI 42.6-63.6), 15 neither fentalog nor heroin $(15.9 \%$, CI $9.2-25)$, and 4 no opioid (4.3\%, CI $1.1-10.5)$. Excluding methadone co-exposures $(N=28)$, mean naloxone total dose $(\mathrm{mg})$ was similar between groups (1.75 fentalog, 1.39 fentanyl, 2.02 heroin, $p=\mathrm{NS}$ ); naloxone continuous infusion rates were similar ( $11.1 \%$ fentalog, $5.5 \%$ fentanyl, $6.1 \%$ heroin, $p=\mathrm{NS}$ ), while repeat naloxone boluses were significantly lower in the fentanyl group $(44.4 \%$ fentalog, $21.1 \%$ fentanyl, $42.9 \%$ heroin, $p<0.05)$. ICU admission rates were similar (14.3\% fentalog, $12.9 \%$ fentanyl, $18.8 \%$ heroin, $p=$ NS), but hospitalization rates were significantly higher for heroin ( $28.5 \%$ fentalog, $38.9 \%$ fentanyl, $45.8 \%$ heroin, $p<0.05$ ).

Conclusion: Most ED patients with suspected heroin OD had confirmed fentanyl/fentalog OD. Treatment needs of fentalog OD were comparable to heroin OD. Post COVID-era studies are ongoing.

\section{Clinical Characteristics of Kratom Exposures Reported to} Georgia Poison Center: a Retrospective Review

Pakhawadee Palungwachira ${ }^{1}$, Michael $\mathrm{Yeh}^{1}$, Ziad Kazzi ${ }^{1}$, William Rushton $^{2}$, Brian Whitworth ${ }^{2}$

${ }^{I}$ Emory university, Atlanta, Georgia, USA. ${ }^{2}$ Alabama poison center, Birmingham, Alabama, USA

Background: Kratom, or Mitragyna speciosa, is a medicinal plant indigenous to Southeast Asia. It has gained attention in the United States for its increased use as a stimulant with opioid-like effects, as well as for concerns about its safety. We describe the clinical effects of kratom exposure reported to a statewide poison center.

Methods: This is a retrospective analysis of kratom exposures reported to the statewide poison center from January 2017 to June 2020. All cases coded as containing kratom were reviewed for demographic data, clinical effects, and product characteristics. Subjects who reported overdoses or coingestion with non-pharmaceutical xenobiotics were excluded. Cases were stratified into three groups according to self-reported dose of kratom ingested within the last $24 \mathrm{hrs}$. Data were then examined for subsets of patients who ingested kratom alone, versus those with reporting therapeutic use of other pharmaceutical products.

Results: One hundred and sixteen cases of kratom exposure were identified. Kratom dosage was reported as low dose (1-5 g, $45.3 \%)$, moderate dose $(5-15 \mathrm{~g}, 18.1 \%)$ and high dose (>15 g, $13.8 \%)$. Most individuals were adults $(91.4 \%)$ who were likely to intentionally $(95.3 \%)$ abuse $(70.3 \%)$ commercial kratom products $(99.1 \%)$. The two most common clinical effects after kratom exposure were central nervous system excitation (single-substance; $25.7 \%$, multiple-substance; $47.8 \%$ ) and tachycardia (single-substance; $47.7 \%$, multiple-substance; 39.5\%). Multiple-substance exposures accounted for $39.7 \%$ of cases and were positively associated with admission to a hospital floor $(21.7 \%$ vs. $12.9 \%)$, intensive care unit $(30.4 \%$ vs. $10.0 \%)$, and serious medical outcomes $(30.4 \%$ vs. $10.0 \%)$ compared to single-substance exposures ( $p$ value $<0.05$ ). There was no association between kratom dose on clinical effects or outcomes.

Conclusion: Kratom exposure is associated with a variety of neurologic and cardiovascular symptoms. Individuals should be educated about its potential risks associated with more serious medical outcome associated with its use in combination with other substances.

\section{Respiratory Failure and Naloxone Use in Confirmed Synthetic Cannabinoid Overdose}

Alex F Manini ${ }^{1,2}$, Alex J Krotulski ${ }^{3}$, Jonathan Schimmel ${ }^{4}$, Lisa Allen ${ }^{1}$, Yasmin L Hurd ${ }^{1}$, Lynne D Richardson ${ }^{1}$, Kavey Vidal ${ }^{1}$, Barry K Logan ${ }^{3,5}$ ${ }^{I}$ Icahn School of Medicine at Mount Sinai, New York, NY, USA. ${ }^{2}$ Elmhurst Hospital Center, New York, NY, USA. ${ }^{3}$ Center for Forensic Science Research \& Education; Fredric Rieders Family Foundation, Willow Grove, PA, USA. ${ }^{4}$ Department of Emergency Medicine; Icahn School of Medicine at Mount Sinai, New York, NY, USA. ${ }^{5}$ NMS Labs, Horsham, PA, USA

Background: The endocannabinoid and endogenous opioid systems are synergistic and agonists targeting both possess shared pharmacological effects including sedation and hypoventilation. Acute respiratory failure 
(ARF) from synthetic cannabinoid (SC) overdose is described but poorly characterized, limited to uncontrolled small case series lacking analytical confirmation.

Research Question: The objective is to describe ARF incidence in ED patients with confirmed SC exposure, and to investigate associations with ARF compared to non-SC overdose.

Methods: This was a convenience cohort of ED patients $\geq 18$ years with suspected SC overdose between 2015 and 2020 at two tertiary-care hospitals. All overdoses were screened, and were excluded if waste serum was unavailable or SC drugs were not suspected based on chart review. Serum was analyzed via liquid chromatography/quadrupole time-offlight mass spectrometry, for $>800$ drugs including novel psychoactive substances. Trained abstractors performed chart review. The primary outcome was in-hospital ARF, defined as (A) intubation, (B) non-invasive ventilation, or (C) naloxone administration. Secondary outcomes included adverse cardiovascular events, ED disposition, and in-hospital mortality.

Results: Of 84 ED patients with suspected SC overdose, there were 29 confirmed SC exposures (12 sedative co-exposures, 6 opioid co-exposures) and 56 confirmed non-SC overdoses. Confirmed SC drugs were: 5F-MDMB-PICA (18), its metabolite 5OH-MDMB-PICA (16), ADBFUBINACA (4), AB-CHIMINACA (4), AB-FUBINACA (1), ABPINACA (1), MDMB-4en-PINACA (1), 4F-MDMB-BINACA (1). ED disposition was $8.3 \%$ ICU; $27.4 \%$ hospitalization; $72.6 \%$ discharge. ARF incidence was $30.9 \%$ (95\% CI 21-41), cardiovascular events in $2.3 \%$ (95\% CI 0.3-8.3), and no deaths. Excluding those with opioid/sedative co-exposure, SC overdose was significantly associated with ARF $(25.0 \%$ $\mathrm{SC}$ vs. $4.2 \%$ non-SC, $p=0.05)$. Naloxone was administered to seven patients with SC exposure, only two of whom had opioid co-exposure.

Conclusion: Confirmed SC overdose was significantly associated with ARF. Use of naloxone for SC respiratory depression has been previously reported, and if confirmed in larger cohorts would warrant further study.

\section{Heroin Overdoses in the Teenage Population}

Saumitra Rege, Heather Borek, Avery Michienzi, Christopher Holstege University of Virginia, Charlottesville, VA, USA

Background: Heroin use in the United States has increased significantly with over 15,000 deaths in the year 2018 . We sought to characterize the heroin exposures among the teenage population reported to the U.S. poison centers (PCs).

Methods: The National Poison Data System (NPDS) was queried for all teenage exposures to heroin reported to the U.S. PCs between 2008 and 2018. Cases that resulted in fatalities or major medical outcomes were classified as serious adverse events (SAEs). We descriptively assessed the demographic and clinical characteristics. Trends were analyzed using Poisson regression. Independent predictors of SAEs were studied using multivariable logistic regression with adjusted odds ratios (AOR) reported.

Results: There were 4,619 teenage heroin exposure calls made to the PCs from 2008 to 2018 , with the proportion of annual exposures with SAEs increasing from $17.5 \%$ to $28.1 \%$. Single substance exposures accounted for $57.8 \%$ of heroin exposures with SAEs. Among the SAE exposures, $62 \%$ were male; intentional abuse $(82.1 \%)$ and suspected suicides $(7.8 \%)$ were commonly observed reasons for exposure. The most frequently co-occurring substance was benzodiazepines $(12.9 \%)$. During the study period, the rate of heroin exposures with SAEs increased from 28.5 to 53.8 (per 100,000 teenage exposures) $(p<0.001)$. Males (Ref: females) (AOR: $1.18,95 \%$ CI: $1.01-1.41$ ) were at a significantly higher risk of SAE. Other factors that increased the odds of SAEs were multisubstance exposures (Ref: single substance exposures) (AOR: 1.26, 95\% CI: 1.01-1.57), parenteral routes of exposure (Ref: ingestion)
(AOR: 2.43, 95\% CI: 1.85-3.20), and intentional abuse (Ref: unintentional exposures) (AOR: 1.60, 95\% CI: 1.19-2.16).

Conclusion: There was a significant increase in the reports of teenage heroin exposures with SAEs during the study. Several key characteristics, including reasons for exposure and presence of multiple substances in exposure, significantly increased the risk of SAE.

\section{Day 3: Lightning Orals, Abstracts 029-035}

029. A Prospective Observational Study of Intramuscular Medications to Treat Agitation due to Methamphetamine Intoxication in the Emergency Department

Jon B Cole ${ }^{1,2}$, Paige A De Vries ${ }^{1}$, Jamie L Stang ${ }^{1,3}$, Ann M Arens ${ }^{1,2}$, Travis D Olives ${ }^{1,2}$, Marc L Martel ${ }^{1}$, James R Miner ${ }^{1,3}$, Brian E Driver ${ }^{1}$ ${ }^{1}$ Hennepin Healthcare, Minneapolis, MN, USA. ${ }^{2}$ Minnesota Poison Control System, Minneapolis, MN, USA. ${ }^{3}$ University of Minnesota Medical School, Minneapolis, MN, USA

Background: Emergency department (ED) visits for acute methamphetamine-associated agitation are increasing; many cases require parenteral medications. Medical toxicologists typically recommend benzodiazepines; however, randomized trials of intravenous medications suggest antipsychotics are also effective. Prospective data on intramuscular medications are lacking.

Hypothesis: Both intramuscular antipsychotics and benzodiazepines will be effective for treating methamphetamine-associated agitation in the ED. Methods: This was a prospective observational study conducted from June 2019 to March 2020. Medication and dose were at the discretion of the treating physician. Data acquisition volunteers were trained in the Altered Mental Status Scale (AMSS), an ordinal scale of agitation from -4 (coma) to 0 (normal) to +4 (most agitated). They recorded time to adequate sedation (TAS), defined as $\mathrm{AMSS} \leq 0$, and other clinical data. TAS was analyzed using differences in medians with $95 \%$ confidence intervals (CIs) as well as a Cox proportional hazards model. Secondary outcomes included use of additional (rescue) medications and adverse events.

Results: We enrolled 122 patients with similar baseline characteristics; 37 received droperidol (median dose $5 \mathrm{mg}$, TAS $=16$ minutes, IQR 10 30), 44 received olanzapine (median dose $10 \mathrm{mg}, \mathrm{TAS}=16$ minutes, IQR 11-29), 15 received midazolam (median dose $5 \mathrm{mg}$, TAS $=13$ minutes, IQR 6-19), and 26 received lorazepam (median dose $2 \mathrm{mg}$, TAS $=29$ minutes, IQR 12-49). Proportional hazards analysis showed lorazepam was associated with slower TAS $(p<0.001)$. The proportion of patients adequately sedated at 15 minutes was $43 \%$ for droperidol, $45 \%$ for olanzapine, $60 \%$ for midazolam, and $32 \%$ for lorazepam. The proportion of patients needing rescue sedation was $16 \%$ for droperidol, $20 \%$ for olanzapine, $13 \%$ for midazolam, and $46 \%$ for lorazepam. We found no difference in adverse events.

Conclusion: Droperidol, olanzapine, and midazolam resulted in similar times to adequate sedation when treating methamphetamine-associated agitation; all three medications provided more rapid sedation than lorazepam.

030. National Poison Data System (NPDS) Review of Intentional Sodium Nitrite Ingestions 2009-2019: A Concerning Trend

Anita Mudan ${ }^{1,2}$, Jacob A Lebin ${ }^{1,2}$, Craig G Smollin ${ }^{1,2}$

${ }^{1}$ Department of Emergency Medicine, University of California San Francisco, San Francisco, CA, USA. ${ }^{2}$ California Poison Control System - San Francisco, San Francisco, CA, USA

Background: Sodium nitrite has many uses, including rubber manufacturing, food preservation, and as a treatment for cyanide toxicity. 
Recently, ingesting sodium nitrite has appeared to gain popularity as a method of committing suicide, spurred by online suicide blogs and the ease of obtaining the product. However, the exact nature of this trend has not been studied.

Hypothesis: We hypothesize that intentional sodium nitrite ingestions will have significantly increased over recent years

Methods: We conducted a 10-year retrospective review of all intentional sodium nitrite ingestion cases reported to the National Poison Data System from January 1, 2009, through December 31, 2019. All cases coded as "nitrite or nitrate," regardless of the exact product, intent, or type of exposure, were included in the data request. Full case records were requested to confirm case details.

Results: There were 390 individual "nitrite or nitrate" exposures reported to the NPDS during the time period, 114 of which were intentional, and 42 were intentional-suspected suicide or unknown. Full case records were received for $35 / 42$ cases, and 17 cases met final study inclusion criteria. The average age was 23.2 years old, and the cohort was evenly split between male and female patients. The majority of ingestions were more than 15 grams. A majority of patients $(13 / 17,76.4 \%)$ presented with visible cyanosis with an average oxygen saturation of $85 \%$. Methylene blue was administered in $82 \%(14 /$ 17) of cases with $47.1 \%$ (8/17) patients requiring advanced cardiac life support. In eight cases, it was confirmed that patients had researched this specific suicide method online. The overall mortality rate in the cohort was $41.2 \%$ (7/17). All cases occurred in the final two years of the study period.

Conclusion: Intentional sodium nitrite ingestion represents a novel and growing trend in attempted suicide methodology and confers a high mortality rate among young adults.

\section{Anti-Epileptic Drug Exposures Among Children and Adolescents, 2000-2020}

\section{Michael S Toce, Florence Bourgeois \\ Boston Children's Hospital, Boston, MA, USA}

Background: Children and adolescents are increasingly prescribed antiepileptic drugs (AEDs) for psychiatric indications, exposing many children to potentially harmful medications, including adolescents at highrisk for self-harm.

Research Question: What is the epidemiology of pediatric AED poisoning and which are associated with severe clinical outcomes?

Methods: This is a retrospective cross-sectional analysis of AED exposures in patients $<20$ years reported to the National Poison Database System (NPDS), 2000-2020. We used descriptive statistics to characterize the study population, negative binomial regression to assess exposure trends, and logistic regression to test the association between patient-level characteristics and serious clinical outcomes.

Results: There were 220,229 AED exposures during the study period, with the yearly case rate increasing by $38.4 \%$ (95\% CI 26.5 $51.6 \%$ ). Cases peaked in 2009 with 14.6 exposures per 100,000 persons then leveled off. The most commonly reported exposures were clonazepam (3.03 exposures per 100,000 persons), valproic acid (1.47), gabapentin (1.44), lamotrigine (1.34), and carbamazepine (1.17). Twenty-five thousand $(11 \%)$ patients were admitted to the hospital, including $6 \%$ to a critical care unit. Twelve hundred (1\%) patients seized, $1,535(1 \%)$ were intubated, and 18 died. Compared to all other AEDs, carbamazepine was associated with greatest risk for hospital admission (OR 3.4, 95\% CI 3.3-3.5) or intubation (OR 8.3, 95\% CI 7.5-9.3). Tiagabine was associated with the greatest risk for seizure $(13.8,95 \%$ CI 10.3-18.6). Adolescents 15-19 years were more likely to be admitted (OR 3.4, 95\% CI 3.33.5 ), intubated (OR 4.1, 95\% CI 3.7-4.6), or seized (OR 3.2, 95\% CI 2.8-3.7) compared to children <five years, likely reflecting larger, intentional ingestions as opposed to exploratory ingestions.
Conclusion: Pediatric AED poisonings are increasing, with carbamazepine and tiagabine associated with more severe outcomes. While results need to be interpreted within known reporting biases of NPDS data, our findings highlight important risks of AEDs particularly among adolescents.

\section{Referral Sources for Substance Use Treatment Admissions among Pregnant Women in the United States}

Laurie S Halmo ${ }^{1,2}$, Karilynn Rockhill ${ }^{2}$, Malin Rapp Olsson ${ }^{2}$, George S Wang $^{1,2}$, Janetta Iwanicki ${ }^{2}$

${ }^{1}$ University of Colorado School of Medicine, Aurora, Colorado, USA.

${ }^{2}$ Rocky Mountain Poison and Drug Safety, Denver, Colorado, USA

Background: Substance use during pregnancy legally constitutes child abuse in 23 states and the District of Columbia. Qualitative studies have suggested these laws may deter pregnant women from seeking prenatal and substance-use care.

Research Question: Is the proportion of pregnant women referred by healthcare providers to substance use treatment facilities smaller in states where substance use during pregnancy constitutes child abuse?

Methods: This was a retrospective review of admissions of pregnant women aged 15-49 years from 2012 to 2017 in the Treatment Episode Data Set - Admissions (TEDS-A), a database of admissions to all publicly funded substance use treatment facilities across the United States. Referral sources for women living in participating states where substance use during pregnancy constitutes child abuse were compared to those for women living in all other states. States were categorized as having or not having such laws according to the Guttmacher Institute's published lists for each year. Differences in proportions of referral source by state laws were calculated with chi-square tests.

Results: In states where substance use during pregnancy constitutes child abuse compared to states without these laws, a smaller proportion of pregnant women entering substance use treatment facilities was referred by a healthcare provider $(14.6 \%$ vs $19.1 \%, p<0.001)$, and self-referred (31.6\% vs. $36.8 \%, p<0.001)$, respectively. In contrast, a larger proportion of pregnant women in states with these laws was referred via the criminal justice system $(26.9 \%$ vs. $24.4 \%, p<0.001)$ and through schools, employers, and community referrals (such as shelters or religious organizations; $24.5 \%$ vs. $17.3 \%, p<0.001$ ) compared to states without such laws.

Conclusion: A smaller proportion of pregnant women requiring substance use treatment was referred for admission by a healthcare provider in states where substance use during pregnancy constitutes child abuse, suggesting a need for novel approaches to connect substance-using pregnant women in these states to medical care.

033. Blood Lead Levels and Risk of Deaths From Cardiovascular Disease: An 18-Year Follow-Up of a National Cohort

Madison K Cook ${ }^{1}$, Yudan Wei ${ }^{2}$, Jian Zhang ${ }^{3}$

${ }^{1}$ Mercer University School of Medicine, MD program, Macon, GA, USA.

${ }^{2}$ Mercer University School of Medicine, Department of Community Medicine, Macon, GA, USA. ${ }^{3}$ Georgia Southern University, Department of Epidemiology, Statesboro, GA, USA

Background: Environmental exposure could be an important modifiable risk factor but is often overlooked in terms of cardiovascular disease (CVD) prevention. Multiple studies have shown a correlation between heavy metal exposures, specifically lead, and CVD risk.

Research Question: In this study, we further investigated the relationship between lead exposure in the general population, measured as blood lead levels (BLL), and mortality from CVD in a national cohort. 
Methods: A total of 15,036 adults aged 19 or older who participated in the National Health and Nutrition Examination Survey (1988-1994) as the baseline examination were followed up through December 31, 2010. Adjusted hazard ratios (HRs) derived from Cox proportional hazard regression models were used to estimate the risk of dying from all CVD and CVD specific disease in association with BLLs.

Results: The median survival time for CVD was significantly shorter in adults in the high blood lead quartile (15.41 years) than in the low lead quartile (16.72 years). The cardiovascular mortality was $3.76,8.11$, and $14.77 / 1,000$ person-years for the individuals in low, intermediate, and high lead quartiles, respectively. The adjusted HRs of all CVD deaths in adults with intermediate and high lead quartiles were $1.10(0.93,1.31)$ and 1.35 $(1.13,1.61)$, respectively, as compared to the low lead quartile. The adjusted HRs of deaths from heart disease in adults with intermediate and high lead quartiles were $1.25(1.03,1.51)$ and $1.51(1.23,1.84)$, respectively. The estimated association with all CVD and heart disease deaths remained significant when analyzing BLLs as continuous variable (log-transformed).

Conclusion: The study demonstrates that increasing BLLs were associated with an increased risk of cardiovascular deaths, especially from heart disease. This further supports the possible cardiovascular effects that lead poses on individuals and the importance of monitoring lead levels and reducing lead exposure in the general population.

\section{Does Patiromer Bind Lithium? An In-vitro Study}

David B Liss, Stephen Roper, Dennis Dietzen, Michael E Mullins Washington University, Saint Louis, Missouri, USA

Background: The AAPCC NPDS reported 7055 lithium carbonate exposures and three deaths in 2018. Lithium toxicity may warrant hemodialysis, which may not be immediately available in some settings. Patiromer is an oral potassium binding agent used for hyperkalemia. It is unknown whether patiromer binds lithium $\left(\mathrm{Li}^{+}\right)$.

Hypothesis: To determine whether patiromer will bind $\mathrm{Li}^{+}$ions in-vitro at a concentration of clinical interest $(>/=2.5 \mathrm{mmol} / \mathrm{L})$.

Methods: We added normal saline (NS) to $6 \mathrm{~mL}$ green-top lithium-heparin $(\mathrm{LiH}) \mathrm{BD}$ Vacutainer ${ }^{\circledR}$ (Franklin Lakes, NJ) tubes to simulate serum. We tested different volumes of NS to identify target volumes producing an apparent $\left[\mathrm{Li}^{+}\right]$of $>/=2.5 \mathrm{mmol} / \mathrm{L}$. Addition of $0.50,0.75$ and $1.0 \mathrm{~mL}$ of NS to $\mathrm{LiH}$ tubes produced apparent $\left[\mathrm{Li}^{+}\right]$of $4.20-4.90,3.04-3.94$, and $2.99-3.21$ $\mathrm{mmol} / \mathrm{L}$, respectively. We added $250 \mathrm{mg}$ of patiromer to $50 \mathrm{~mL}$ of NS in a conical vial and suspended by vortex mixing. We added aliquots of $0.4,0.8$, $2.0,4.0$, and $20 \mathrm{~mL}$ of this stock suspension to conical vials and volume balanced with NS to a total of $20 \mathrm{~mL}$, achieving concentrations of $0.1,0.2$, $0.5,1.0$, and $5.0 \mathrm{mg} / \mathrm{mL}$ of patiromer in NS. We vortex mixed the patiromer suspensions, and pipetted aliquots of $0.5 \mathrm{~mL}, 0.75 \mathrm{~mL}$, and $1 \mathrm{~mL}$ of each suspension into the $\mathrm{LiH}$ Vacutainer ${ }^{\circledR}$ tubes (two tubes for each concentration). We placed the tubes on a rocker panel for 10 minutes, and centrifuged for five min at $4400 \mathrm{rpm}$. We pipetted 350 microliters of the supernatant from each into $1.5 \mathrm{~mL}$ microcentrifuge tubes and measured $\mathrm{Li}^{+}$and sodium $\left(\mathrm{Na}^{+}\right)$ using a Roche® 9180 Electrolyte Analyzer with an ion selective electrode.

Results: Increasing concentrations from 0 to $5.0 \mathrm{mg} / \mathrm{mL}$ of patiromer had no effect on $\mathrm{Li}^{+}$concentrations.

Conclusion: Patiromer does not reduce the lithium concentrations in this in-vitro model.

035. Analysis of Smoke-Related Calls to the Oregon Poison Center During the September 2020 Wildfires

Matthew S Correia ${ }^{1,2}$, B Zane Horowitz ${ }^{3,4}$

${ }^{1}$ Oregon Health and Science University, Portland, Oregon, USA. ${ }^{2}$ Oregon Poison Center, Portland, Oregon, USA. ${ }^{3}$ Oregon Health and Science University, Portland, OR, USA. ${ }^{4}$ Oregon Poison Center, Portland, OR, USA
Background: Uncharacteristically high winds and an unusual dry summer facilitated explosive growth of numerous fires smoldering throughout Oregon during early September 2020. Unfortunately, the winds which led to their rapid growth quickly abated and low-level air flow stagnated for nearly two weeks as a cut-off low pressure system remained off the Pacific Coast. Dense, smoke-laden air inundated much of the western half of the state and led Portland to experience the worst air quality of all large cities in the entire world, measured by PM 2.5, for nearly two weeks. Air quality for much of the state west of the Cascades fell within the environmental protection agency's rating of 'hazardous' as noted by an AQI > 300. Many localities suffered AQI's > 500 for many days.

Methods: We conducted a retrospective chart review of Wildfire related Oregon Poison Center calls for September 2020. Search Terms included 'Smoke', 'Fire', and 'Carbon'. Records were evaluated on a case-by-case basis. Cases coded as 'Carbon Monoxide' were screened based on contextual data to assess whether they were smoke-related or attributable to another etiology (i.e. indoor use of campfire stoves). Extracted data included call date, patient age, sex, symptoms and caller zip-code to enable comparative geomapping.

Results: There were 58 wildfire-smoke related calls all clustered over a 12-day period between September 6-18 where PM 2.5 maps noted much of the state was experiencing very poor air quality. Only four calls originated from a healthcare facility. Calls were evenly split among sex. Many calls were informational in nature specifically concerning the risk of carbon monoxide exposure.

Conclusion: There was a clear temporal relationship between poor outdoor air quality and poison center calls. Future education should focus on conveying the low risk of carbon monoxide toxicity unless in very close proximity to smoldering fire.

\section{Day 3: Moderated Posters, Abstracts 036-040}

\section{Efficacy of a Substance Abuse Screening Tool in the Emergency Department}

Ryan Kramer ${ }^{1}$, Scott T Richardson $\mathrm{Jr}^{1}$, Diane T Dawley ${ }^{1}$, Clayton Cook ${ }^{1}$, Allison L Mainhart ${ }^{1}$, Lawrence Greenblatt ${ }^{2}$, Jennifer L Parker Cote ${ }^{1}$ ${ }^{1}$ East Carolina University-Brody School of Medicine, Greenville, NC, USA. ${ }^{2}$ Duke University School of Medicine, Durham, NC, USA

Background: There are mixed results in regard to the effectiveness of substance abuse screening and counseling in the emergency department (ED) setting. The Tobacco, Alcohol, and Prescription Medication, and Other Substance use (TAPS) tool was initially developed for substance abuse screening in the adult primary care setting. The aim of this pilot study was to assess the efficacy of TAPS tool in the emergency department by screening adult patients in the emergency department, providing outpatient resources, and assessing the number of patients who followedup with an addiction resource.

Methods: A convenience sample of adult patients in the ED were screened for substance abuse over the course of two weeks with the TAPS tool. The TAPS tool consists of two sections. If a patient was positive on TAPS-1, they then completed TAPS- 2 screening. Patients who score $2+$ were provided a list of outpatient resources for substance abuse treatment. Approximately two to four weeks after screening patients were followed up via phone survey and asked if they contacted (call, email, or visit) an addiction resource.

Results: A total of 117 patients were screened. 62 patients screened positive on TAPS-2 and were provided with outpatient addiction resources. Twenty-three patients did not complete the follow-up survey: screeners were unable to contact 16 patients after three attempts, and seven patients refused follow-up after screening. Screeners contacted 39 patients for follow-up. Twelve (30.8\%) of the 39 patients contacted addiction resources. 
Twenty-four $(61.5 \%)$ did not contact an addiction resource. Three preferred not to answer the follow-up question when called.

Conclusion: In this pilot study, 53\% of patients screened positive for substance abuse in the ED. Of those screening positive, $30.8 \%$ contacted an addiction resource after being provided resources. This pilot study suggests screening for substance abuse and providing addiction resources may be beneficial in the ED.

037. Bounce Back Rates in Discharged Emergency Department Patients Treated with Phenobarbital Versus Benzodiazepines for Alcohol Withdrawal

Jacob A Lebin, Anita Mudan, Charles E Murphy IV, Ralph Wang, Craig G Smollin

Department of Emergency Medicine, University of California, San Francisco, CA, USA

Background: Alcohol withdrawal is a common presentation to the emergency department (ED). Phenobarbital has been successfully used as a primary medication to manage alcohol withdrawal, but the drug's long half-life may result in delayed respiratory depression. Few studies have examined clinical outcomes in discharged ED patients who receive phenobarbital for alcohol withdrawal.

Hypothesis: We hypothesized that discharged ED patients who received phenobarbital $+/-$ benzodiazepines for alcohol withdrawal would have lower ED bounce back rates compared to patients who received benzodiazepines alone.

Methods: This was a single center, retrospective cohort study of consecutive patients discharged from the ED with a diagnosis of alcohol withdrawal after receiving either phenobarbital $+/-$ benzodiazepines or benzodiazepines alone. The primary outcome was ED bounce back within three or seven days from the index ED visit. Secondary outcomes included short-term survival beyond or death within three days from the index ED visit as confirmed by chart review or the county medical examiner.

Results: There were 470 patient encounters with 235 (50\%) receiving phenobarbital. The median phenobarbital dose was $390 \mathrm{mg}$ (IQR: 260 $520 \mathrm{mg}$ ). Patients who received phenobarbital were less likely to bounce back to the ED within three days compared to patients who received benzodiazepines $(11.4 \%$ vs. $24.6 \%, 95 \% \mathrm{CI}=6.3 \%$ to $20.0 \%, p=0$ $.0002)$, but this difference normalized for bounce backs between three and seven days ( $7.2 \%$ vs. $7.6 \%, p=\mathrm{NS})$. Patients treated with phenobarbital were less likely to bounce back for alcohol withdrawal (3.8\% vs. $9.8 \%, 95 \% \mathrm{CI}=1.4 \%$ to $10.8 \%, p=0.01)$. Documented short-term survival was high in both cohorts ( $95 \%$ vs. $94 \%, p=$ NS) and there were no deaths.

Conclusion: Patients who received phenobarbital for alcohol withdrawal were less likely to bounce back within three days of ED discharge. Despite the long half-life, the outpatient safety of phenobarbital appears similar to benzodiazepines.

038. Influence of Pennsylvania Liquor Store Closures during the COVID-19 Pandemic on Features of Alcohol Withdrawal Syndrome Consultations

Alexandra M Amaducci, Ali R Yazdanyar, Derek J Fikse, Jasdip Kaur, Andrew L Koons, Gillian A Beauchamp, Robert D Cannon, Ryan M Surmaitis, Matthew D Cook, Kenneth D Katz

Lehigh Valley Health Network/USF Morsani College of Medicine, Allentown, PA, USA

Background: Alcohol withdrawal syndrome (AWS) is a serious consequence of alcohol use disorder. Due to the current COVID-19 pandemic, many businesses were shuttered-including a statewide closure of Pennsylvania liquor stores on March 17, 2020.
Hypothesis: As a result of statewide liquor store closures, an increase in AWS consultations to medical toxicology would occur.

Methods: This is a retrospective, observational study of AWS patients presenting to a tertiary care hospital. We calculated descriptive statistics for continuous and categorical variables and compared AWS consults placed to the medical toxicology service for five months preceding liquor store closure to those placed between March 17, 2020, and August 31, 2020. We evaluated Emergency Department (ED) length of stay (LOS), weekly and monthly consultation rate, and rate of admission and ED recidivism both pre- and post-liquor store closure.

Results: A total of 324 AWS consults were placed during the 10month period. One hundred and forty-two consults $(43.8 \%)$ were pre- and $182(56.2 \%)$ were post-liquor store closure. There was no significant difference in patient age, gender, or race, nor weekly or monthly consultation rate when comparing pre- and post-liquor store periods. The median ED LOS was seven hours, 95\% Confidence Interval (CI) $[5,10]$, and did not significantly differ between the pre- and post-liquor store periods $(p=0.78) .92 .9 \%$ of the patients presenting with AWS required an admission with no significant difference between the pre- and post-liquor store closure periods $(94.4 \%$ vs. $91.8 \%, p=0.36)$. Following liquor store closure, there was a significant increase in the number of AWS patients requiring a return ED visit: Odds Ratio 2.49; 95\% CI [1.38, 4.49].

Conclusion: There was nearly two and a half fold greater odds of ED recidivism among post-liquor store closure patients with AWS as compared with the pre-closure patients.

039. Impact of the COVID-19 Pandemic on Emergency Department Substance Use Presentations and Substance Use Screens

Kira Chandran ${ }^{1}$, Maryann Mazer-Amirshahi ${ }^{2}$, Nikash Shankar ${ }^{1}$, Sameer Desale $^{3}$, Lewis S Nelson ${ }^{4}$, Mihriye Mete ${ }^{3}$

${ }^{1}$ Georgetown University School of Medicine, Washington, DC, USA. ${ }^{2}$ MedStar Washington Hospital Center, Washington, DC, USA. ${ }^{3}$ MedStar Health, Colombia, MD, USA. ${ }^{4}$ Rutgers University, Newark, NJ, USA

Background: The COVID-19 pandemic has adversely affected vulnerable patient populations and can exacerbate substance use disorder. There are limited data regarding how this has impacted emergency department (ED) patient presentations.

Hypothesis: There would be an increase in the proportion of patients presenting with acute alcohol or other substance intoxication and patients presenting with a positive screen for substance use during the COVID-19 pandemic compared to the months prior.

Methods: Retrospective chart review using data from nine hospitals within one health system in the DC/Baltimore area of ED visits between 11/1/2019 and 6/30/2020. The health system utilizes a validated screening system for Screening, Brief Intervention, and Referral to Treatment (SBIRT) for ED patients who are clinically stable and willing to complete screening. We evaluated trends in patients with positive SBIRT screens and those presenting with acute alcohol or substance intoxication/overdose before (11/1/19-2/28/2020) and during the peak of the COVID pandemic (3/1/2020-6/30/2020). Data were described using summary statistics. Bivariate analyses were conducted using chi-square test.

Results: There were 163,462 screens performed during the study period (93,253 pre-COVID, 70,209 during). The population was primarily blAck $(60.7 \%)$ and $55.7 \%$ were female. Positive SBIRT screens increased from $11.5 \%$ pre-COVID to $14.4 \%$ during COVID $(p<0.001)$. Alcohol intoxication presentations increased as a proportion of positive screens from $1.3 \%$ to $1.9 \%,(p<0.001)$. Substance intoxications/ overdoses increased as a percentage of positive screens during the pandemic $(1.0 \%$ to $1.6 \%, p<0.001)$. The proportion of opioid $(60.2 \%)$ vs. 
non-opioid (39.8\%) overdoses remained unchanged. A higher percentage of screened patients reported problem drinking (AUDIT-C score $\geq 7$ ) during the pandemic $(2.7 \%$ vs. $3.4 \%, p<0.001)$.

Conclusion: There was an increase in the proportion of positive SBIRT screens and visits for acute overdoses and intoxication during the COVID-19 pandemic. Additional research should focus on mitigation strategies to address substance use during this vulnerable time.

040. Low-Threshold 24/7 Buprenorphine Telehealth Treatment: Innovation to Improve Access During COVID-19

Rachel S Wightman ${ }^{1}$, Caroline Wunsch ${ }^{1}$, Seth Clark $^{1}$, Lee Ann Jordison Keeler $^{2}$, Laureen Berkowitz ${ }^{3}$, Justin Berk ${ }^{1}$, Elizabeth A Samuels ${ }^{1}$ ${ }^{1}$ Alpert Medical School of Brown University, Providence, RI, USA. ${ }^{2}$ Brown Emergency Medicine, Providence, RI, USA. ${ }^{3}$ Remedy 180 Center, Pawtucket, RI, USA

Background: Covid-19 and long-standing barriers to treatment heighten the risk for opioid overdose. In response to the COVID-19 pandemic, the Substance Abuse Mental Health Services Administration (SAMHSA), the US Drug Enforcement Administration (DEA), and the US Department of Health and Human Services changed federal guidance to allow for use of telehealth for buprenorphine initiation without requiring an in-person evaluation or video interface. These regulatory changes created a unique opportunity to trial a low-threshold, audio-only buprenorphine telehealth treatment model.

Methods: In collaboration with the Rhode Island Department of Health and the Rhode Island Department of Behavioral Health, Developmental Disabilities, and Hospitals, we established the Rhode Island Buprenorphine Hotline, a statewide on-demand buprenorphine telephone treatment line. This hotline functions as a 24-hour tele-bridge clinic where people with moderate to severe opioid use disorder are connected with a DATA 2000 waivered provider for an initial assessment, and, if appropriate, obtain a prescription for unobserved buprenorphine initiation with linkage to outpatient maintenance treatment, naloxone, and other harm reduction resources.

Results: From April to September 2020, 87 patients with OUD were evaluated for buprenorphine treatment initiation via the hotline. Thirtyfive out of $63(55 \%)$ were in opioid withdrawal at the time of the call. Forty out of $63(63 \%)$ had previously taken buprenorphine without a prescription. Fifty-eight out of $63(92 \%)$ of patients were not enrolled in SUD treatment at the time of call and many expressed difficulties accessing treatment. Sixty-nine patients were started on buprenorphine via an unobserved treatment protocol and linked to ongoing maintenance treatment.

Conclusion: Although further evaluation is needed, this hotline is one common-sense strategy to address a complex treatment access problem and provides valuable lessons for improving access to care. Future evaluations are needed to evaluate acceptability and comparative efficacy to in-person encounters.

\section{All Posters, Abstracts 041-178}

041. pH Miracle pHour Salt Ingestion Leading to Acute Renal Failure, Caustic Injury and Metabolic Alkalosis

Lauren M Porter, $\mathrm{DO}^{1,2}$, Marlea A Miano, $\mathrm{MD}^{1,2}$

${ }^{1}$ University Hospitals Center for Emergency Medicine, Cleveland, $\mathrm{OH}$, USA. ${ }^{2}$ Case Western Reserve University, Cleveland, OH, USA

Background: Alkaline supplements including pH Miracle pHour Salts are popular due to false claims of homeopathic remedies despite lack of clinical evidence. They can be found through various Internet sources.
Methods: This is a single case report. A 38-year-old male with a history of chronic pancreatitis and alcohol use presented for abdominal pain and was diagnosed with pancreatitis and discharged. He returned three days later due after ingesting 2 doses of pHour Salts and was found to have acute hemorrhagic and necrotizing pancreatitis with an associated highgrade bowel obstruction, possible duodenal perforation with a surrounding hematoma. His labs had worsened with a lipase of $7958 \mathrm{U} / \mathrm{L}$ from 1795. His VBG was 7.62/51/42/52.4 and renal function had declined with a BUN/Cr 42/3.04 from 9/0.81 and electrolytes were $\mathrm{Na} \mathrm{139,} \mathrm{K} \mathrm{2.9,} \mathrm{Cl}$ $70, \mathrm{HCO} 353$, phosphorus 5.0 and $\mathrm{Mg} 3.7$. The patient was evaluated by surgery, gastroenterology and toxicology. He received fluids and an NG tube. EGD showed a large ulcer in the duodenal bulb with surrounding hematoma. Omeprazole was started and he was discharged on HD 7. Repeat endoscopy showed improved gastritis.

Conclusion: pHour Miracle Salts Powder consists of $32 \mathrm{mg} \mathrm{Ca}, 20 \mathrm{mg}$ $\mathrm{Mg}, 620 \mathrm{mg} \mathrm{Na}$ and $814 \mathrm{mg} \mathrm{K}$ in a $5 \mathrm{~g}$ serving. It is marketed to improve the health of various organ systems. Its use led to organ failure and caustic injury. These products should be used with significant caution due to lack of scientific backing of their clinical benefits.

\section{Atypical Case of Severe Potassium Permanganate Poisoning}

Khin Moe Sam ${ }^{1,2}$, Andis Graudins ${ }^{1,2}$

${ }^{1}$ Monash Health, Melbourne, Victoria, Australia. ${ }^{2}$ Monash University, Melbourne, Victoria, Australia

Background: Potassium permanganate is a dermal disinfectant, available over the counter in Australia. We report a case of severe potassium permanganate poisoning.

Research Question: Can severe potassium permanganate poisoning occur in a patient with minimal clinical and metabolic features

Method: This is a single-patient case review. A 55-year-old lady presented to our Metropolitan Hospital $2.5 \mathrm{hr}$ post-ingestion of $25 \mathrm{~g}$ potassium permanganate crystals, sertraline two grams and olanzapine $17.5 \mathrm{mg}$. On arrival, she had mouth, chest and abdominal pain. Vital signs were normal. She had purplish black oral and pharyngeal staining. No pharyngeal swelling or mucosal ulceration, stridor or respiratory distress were evident. There was no acidaemia or kidney injury evident. Mild leucocytosis $\left(12.3 \times 10^{9} / \mathrm{L}\right)$ was the only biochemical abnormality. She was observed overnight with the plan for gastroscopy the next day. Vital signs remained normal and there was no abdominal tenderness.

Results: A gastroscopy performed $26 \mathrm{hr}$ post-ingestion revealed an oedematous oesophagus and black stomach mucosa suggestive of necrosis with antral erythema. Abdominal-CT showed free peritoneal fluid suggesting perforation. Laparotomy revealed discoloured peritoneal fluid, a haemorrhagic oedematous stomach and transverse mesocolon, but no perforation. Direct laryngoscopy revealed vocal cord oedema and the intubation grade was $2 \mathrm{~b}$. Patient was extubated after eight days due to significant airway oedema. She was on Total Parenteral Nutrition for 12 days.

Conclusion: Previous cases of potassium permanganate ingestion describe early onset of clinical and metabolic abnormalities tachycardia, hypotension, airway injury, oral mucosal ulceration, abdominal tenderness metabolic acidaemia, heralding intestinal perforation and kidney failure. In this patient, the clinical and metabolic features were not typical of the reported pattern despite massive ingestion and the subsequent intestinal injury seen on endoscopy and laparotomy. Staining by potassium permanganate may make it difficult to assess mucosal viability and abdominal CT may be a better modality to assess GI injury.

\section{Baclofen Overdose Complicated by Malignant Catatonia Requiring Electroconvulsive Therapy}

Rachael C Westover, Andrew F Micciche, Carin K Malley, Matthew P Scanlon, Joseph H Yanta 
University of Pittsburgh School of Medicine, Pittsburgh, PA, USA

Background: As a GABA-b agonist, baclofen can cause neuroinhibitory and neuroexcitatory toxidromes in both the overdose and withdrawal states. Catatonia is thought to be secondary to reduced GABA-a activity, but increased activity at the GABA-b receptor may worsen catatonia. Hypothesis: Baclofen overdose or withdrawal may contribute to the development of catatonia in susceptible individuals.

Methods: This is a single-patient case report. A 27-year-old man with a history of anxiety, chronic back pain, opioid use disorder, and attentiondeficit disorder was transferred to a tertiary care center for evaluation of encephalopathy after intentional baclofen ingestion requiring ketamine and benzodiazepines for symptomatic control. The patient required high-dose ketamine infusion in addition to as needed phenobarbital and benzodiazepines for agitation management in the ICU. Two weeks later, the patient demonstrated persistent encephalopathy despite negative neurologic, metabolic, rheumatologic, and infectious workups.

Results: The patient had received multiple doses of sedative and antipsychotic agents in addition to physical restraints to control his agitation. His baclofen was restarted with minimal improvement. Psychiatry diagnosed the patient with catatonia secondary to baclofen withdrawal given his clinical course and worsening of symptoms with antipsychotic use; the patient showed initial improvement with high-dose lorazepam but then developed features of malignant catatonia. The patient received one dose of electroconvulsive therapy (ECT) on hospital day 19 with dramatic improvement of his mutism. He continued to improve with ECT treatments three times a week. He was transferred to inpatient psychiatry for further management on hospital day 32 . He is still undergoing ECT one year later.

Conclusion: Baclofen overdose or withdrawal may contribute to the development of catatonia, and prolonged, persistent encephalopathy after overdose should prompt psychiatric evaluation for this disease. Electroconvulsive therapy is the therapeutic modality of choice and is associated with rapid, pronounced improvement in catatonic features.

044. Overdose-induced Vasoplegic Syndrome Treated with Highdose Hydroxocobalamin and Extracorporeal Membrane Oxygenation (ECMO)

Rachel Belcher ${ }^{1}$, Marie Rapier ${ }^{1}$, Alyrene Dorey $^{2}$, Daniel Gutteridge ${ }^{3}$ ${ }^{1}$ Intermountain Medical Center, Department of Pharmacy, Murray, UT, USA. ${ }^{2}$ University of Utah Department of Surgery, Division of Emergency Medicine, Salt Lake City, UT, USA. ${ }^{3}$ Intermountain Medical Center, Division of Pulmonary and Critical Care Medicine, Murray, UT, USA

Background: Management of refractory shock in the setting of overdose can be challenging. Methylene blue and hydroxocobalamin are adjunctive therapies used for vasoplegia after cardiac surgery, which are believed to act on nitric oxide pathways. We describe a case of severe vasodilatory and cardiogenic shock after bupropion and citalopram overdose where vasopressors and high-dose hydroxocobalamin were used successfully to alleviate vasoplegia, allowing placement on VenousArterial Extracorporeal Membrane Oxygenation (VA-ECMO).

Methods: A 23-year-old male was transported to the Emergency Department (ED) after reported overdose of his prescription medications, citalopram and bupropion. Upon arrival to the ED, he sustained a convulsion after which he developed shock, acidemia ( $\mathrm{pH} 7.3$ ) and widecomplex tachydysrhythmias with cardiac arrest. He received sodium bicarbonate, norepinephrine, epinephrine, calcium and return of spontaneous circulation (ROSC) was obtained. Refractory shock post-ROSC persisted despite maximum doses of three vasopressors. Lipid emulsion bolus and drip were delivered with minimal improvement. Subsequently, attempts were made to cannulate for VA-ECMO; however, vascular access was unobtainable due to vascular collapse. Echocardiogram demonstrated an ejection fraction of $15 \%$. Hydroxocobalamin $5 \mathrm{~g}$ was administered and vascular tone rapidly improved, mean arterial pressure increased by $30 \mathrm{mmHg}$, and vascular access was able to be obtained.

Results: The patient was maintained on VA-ECMO for three days. Markedly, elevated serum levels of citalopram $(3400 \mathrm{ng} / \mathrm{mL}$, normal 9$200 \mathrm{ng} / \mathrm{mL})$ and bupropion $(597 \mathrm{ng} / \mathrm{mL}$, normal 50-100) were noted. The patient was discharged to home 28 days after admission.

Conclusion: This unique case demonstrates that high-dose hydroxocobalamin can be effective in refractory vasoplegia and reducing vasopressor requirements in overdose patients needing advanced life saving measures. It is also suggestive that high-dose hydroxocobalamin can be used as an alternative in patients with vasoplegia for whom the use of methylene blue may be contraindicated.

045. Risperidone Induced Antimuscarinic Toxicity Successfully Treated with Physostigmine

Joshua R McFalls ${ }^{1,2}$, Kerollos A Shaker ${ }^{1,2}$, Todd M Phillips ${ }^{2}$

${ }^{1}$ University of Texas Southwestern Medical Center, Dallas, TX, USA.

${ }^{2}$ Parkland Memorial Hospital, Dalllas, TX, USA

Background: Risperidone is a common antipsychotic agent known to have effects on multiple different receptors, but it has not been reported to have significant antimuscarinic effects in known literature. No reports of risperidone-induced antimuscarinic delirium have been reversed by physostigmine.

Hypothesis: Risperidone can cause clinically significant antimuscarinic delirium, and its effects can be reversed using physostigmine.

Methods: This is a case report. A 14-year-old female with history of depression, cutting behavior, and anxiety presented to the ED with altered mental status, combative behavior, tachycardia, and hypotension. Ingestion of unknown amount of unknown medication was suspected when talking with parents. Labs were largely unremarkable and EKG showed narrow complex rhythm and sinus tachycardia in the $150 \mathrm{~s}$. Urine drug screen was negative and comprehensive urine drug testing was sent off. Due to severe hypotension $(60 \mathrm{~s} / 40$ s), she was started on an epinephrine drip and transferred to the PICU. The toxicology team was consulted and bedside evaluation revealed dry mucus membranes, decreased bowel sounds, normal pupil size, and urinary retention. Patient was still altered and required physical restraints with bed ties and multiple nurses holding her down. Decision was made to administer a diagnostic trial of physostigmine.

Results: One mg of physostigmine was given over two minutes. Approximately 2-3 minutes later, she woke up and started asking the care team where she was, back to near neurological baseline. Hypotension and tachycardia gradually improved and epinephrine was weaned. She had recurrence of altered mentation about three hours after physostigmine trial, but did not require chemical intervention overnight. She continued to improve over a three-day hospital course. Comprehensive urine drug testing was positive only for risperidone and metabolites. Patient had a family member with a prescription for risperidone.

Conclusion: Risperidone can cause antimuscarinic delirium that can be successfully treated with physostigmine.

\section{Lurasidone Mono-Ingestion Overdoses: a Case Series with} Minimal Toxicity

Madeleine M Jona ${ }^{1}$, Andis Graudins ${ }^{1,2}$

${ }^{1}$ Monash Health, Melbourne, Victoria, Australia. ${ }^{2}$ Monash University, Clayton, Victoria, Australia

Background: Lurasidone is a second-generation antipsychotic agent. In therapeutic doses, it has few reported side effects, and minimal effect on 
the electrocardiogram (ECG). We describe clinical and ECG features of a series of lurasidone mono-ingestion overdoses.

Method: Retrospective case-series of self-reported lurasidone monoingestion overdoses treated by the Monash Health toxicology unit between 2016 and 2019. Data collected: age, gender, dose of ingested ( $\mathrm{mg}$ and defined daily doses-DDD), vital signs and conscious state, time to lowest GCS, duration of sedation, complications (eg; hypotension, seizures) and ECG abnormalities (rhythm, QRS, QT-heart rate pairs, QTc-Fredericia).

Results: Six patients with mono-ingestion overdose of lurasidone were identified. Sixty-six percent $(n=4)$ were female, median age: 31 years (range: 16-44 years), median reported dose $820 \mathrm{mg}$ (range: $300-2000$ mg), 5-DDDs (range: 1.5-11). Median lowest GCS 14, occurred 2.6 hours (GCS range: 12 to 15 ; time range: 2-9.5 hours) post-ingestion. Hypotension was observed in two patients (median SBP $95 \mathrm{mmHg}$, responsive to fluid). Median heart rate for all patients was $75 \mathrm{bpm}$ (range: 50-112). No patients displayed anticholinergic signs. A total of 13 ECGs were recorded from 1.1 to 15 hours post-ingestion. Median number of ECGs per patient was two. Median QTc(Fredericia) was $425 \mathrm{msec}$ (range: $360-475 \mathrm{msec}$ ). When measured QT-heart rate pairs were plotted on the QT-nomogram, none crossed the nomogram line. Pre-overdose ECGs were available for three patients. QT intervals were similar to postoverdose ECGs. There was no evidence of any other conduction or rhythm disturbances. All patients were admitted to the toxicology observation unit. Median hospital length of stay was 14.4 hours (range: 7.4-22 hours). There were no deaths.

Conclusion: In this small cohort of isolated lurasidone overdoses, mild sedation and minimal ECG interval effects were seen. There was no anticholinergic toxicity, seen after overdose with other atypical antipsychotics, such as quetiapine and olanzapine. Larger case series are required to further confirm features of toxicity.

\section{Duloxetine Poisoning: A Unique Case of Cardiotoxicity}

Derek J Fikse ${ }^{1,2}$, Alexandra M Amaducci ${ }^{1,2}$, Robert D Cannon ${ }^{1,2}$, Kenneth D Katz ${ }^{1,2}$

${ }^{1}$ Lehigh Valley Health Network, Department of Emergency and Hospital Medicine, Division of Toxicology, Allentown, PA, USA. ${ }^{2}$ USF Morsani School of Medicine, Allentown, PA, USA

Background: Duloxetine is a SNRI similar to venlafaxine. While cardiotoxicity from venlafaxine poisoning has been described, it has not previously been reported with duloxetine.

Method: A 15-year-old boy with PMH of depression and anxiety prescribed fluoxetine $20 \mathrm{mg}$ and hydroxyzine $10 \mathrm{mg}$ intentionally ingested $3,060 \mathrm{mg}$ of his mother's duloxetine pills. Notable VS were BP 145/88 and HR 127. On exam, his mental status was normal. He was noted to have six beat ankle clonus and hyperreflexia. CBC, CMP, VBG, salicylate, acetaminophen, and ethanol were unremarkable. Initial EKG demonstrated sinus tachycardia 109 bpm, QRS $103 \mathrm{~ms}$, and QTc $462 \mathrm{~ms}$. One hundred $\mathrm{ml}$ of $8.4 \%$ sodium bicarbonate was administered intravenously without any change in the QRS duration. Serial EKGs demonstrated gradual prolongation of QRS and QTc reaching a maximum of $134 \mathrm{~ms}$ and $543 \mathrm{~ms}$, respectively. Sodium bicarbonate IV was re-administered, again without improvement in the QRS duration. Although his tachycardia and serotonergic signs resolved within 12 hours post ingestion, the QRS widening persisted with gradual resolution over 84 hours to $100 \mathrm{~ms}$. He developed neither hemodynamic instability nor dysrhythmias. Serum LC/MS detected duloxetine, fluoxetine, hydroxyzine, cannabinoids, and caffeine. The patient was admitted to inpatient psychiatry on hospital day five.

Results: The patient was prescribed fluoxetine, a potent CYP450 2D6 inhibitor, which may have inhibited the metabolism of duloxetine, also metabolized by CYP450 2D6. Although in vitro evidence exists demonstrating duloxetine's ability to block cardiac sodium channels, QRS widening or other clinically significant cardiotoxicity has not been described. It is also noteworthy that this patient's QRS interval did not improve after sodium bicarbonate administration. Neither fluoxetine nor hydroxyzine is expected to cause QRS widening.

Conclusion: Physicians should be aware of the potential for duloxetineinduced cardiotoxicity.

\section{QT Prolongation Associated with Co-ingestion of Aripiprazole and CYP2D6 Inhibitor}

Gregory A Makar, Michelle Geyer Thompson, Meghan B Spyres Banner University Medical Center-Phoenix, Phoenix, AZ, USA

Background: Aripiprazole has minimal QT prolonging effects. Fluoxetine, an inhibitor of CYP2D6, impairs metabolism of aripirazole when co-ingested. Cases of aripiprazole associated QT prolongation in the presence of a CYP2D6 inhibitor are not well described.

Hypothesis: Co-ingestion of aripiprazole and CYP2D6 inhibitors can predispose to prolongation of the QT interval.

Methods: Single-patient chart review. A 40-year-old woman presented to an emergency department after intentional ingestion of aripiprazole, fluoxetine and propranolol. Activated charcoal $100 \mathrm{~g}$ was administered. ECG two hours after ingestion showed normal sinus rhythm with QTcF $511 \mathrm{msec}$, corresponding blood pressure $124 / 81 \mathrm{mmHg}$ and heart rate 70 bpm. Seven hours after ingestion, hypotension and bradycardia developed. Norepinephrine, epinephrine and glucagon were initiated, and hypotension improved. Eighteen hours after ingestion, an ECG showed sinus bradycardia with QTcF $616 \mathrm{msec}$; corresponding blood pressure $148 / 81 \mathrm{mmHg}$ and heart rate $46 \mathrm{bpm}$. Isoproterenol was added. At that time, serum aripiprazole level measured $700 \mathrm{ng} / \mathrm{mL}$ (steady-state range $320-585 \mathrm{ng} / \mathrm{mL}$ ). Serum fluoxetine and norfluoxetine levels measured $440 \mathrm{ng} / \mathrm{mL}$ (steady state range $91-302 \mathrm{ng} / \mathrm{mL}$ ) and $300 \mathrm{ng} / \mathrm{mL}$ (steadystate range $72-258 \mathrm{ng} / \mathrm{mL}$ ), respectively. Serum potassium was 4.7 $\mathrm{mmol} / \mathrm{L}$, serum magnesium was $2.5 \mathrm{mg} / \mathrm{dL}$, and serum calcium was 8.0 $\mathrm{mg} / \mathrm{dL}$ (albumin $3.8 \mathrm{~g} / \mathrm{dL}$ ). Over the next 24 hours, the QTcF increased to $670 \mathrm{msec}$ and bradycardia improved (Figure 1); corresponding blood pressure 116/75 $\mathrm{mmHg}$ and heart rate $70 \mathrm{bpm}$. Urine GC/MS confirmed fluoxetine, norfluoxetine, propranolol, caffeine and methamphetamine. TTE demonstrated mildly reduced ejection fraction (45\%) but no structural abnormalities. ECG at the time of discharge revealed normal sinus rhythm with QTcF 472 msec.

Results: Pharmacogenomic testing showed normal function of CYP2D6 and CYP3A4. QT prolongation was greater than expected following aripiprazole ingestion. There was no evidence of structural heart abnormalities, deranged electrolytes, or family history of prolonged QT syndrome or sudden cardiac death. Genetic channelopathy testing is pending. Conclusion: In overdose, co-ingestion of aripiprazole and a CYP2D6 inhibitor may produce significant QT prolongation.

\section{Massive Nortriptyline Poisoning Treated with Extracorporeal Membrane Oxygenation (ECMO)}

Sam T Ontiveros, Alicia B Minns

University of California San Diego, San Diego, California, USA

Background: New indications for tricyclic antidepressants (TCAs) have led to increased prescribing, and overdose remains relevant.

Hypothesis: ECMO may be employed to support patients with massive tricyclic antidepressant poisoning that fail to respond to conventional treatment.

Methods: A 65-year-old female presented to the emergency department after an intentional overdose of nortriptyline. Pre-hospital, the patient was given 70 meq of sodium bicarbonate for a wide complex tachycardia. The patient arrived obtunded with a temperature $90.3 \mathrm{~F}$ and normal 
hemodynamics. She became hypotensive and was given an additional $250 \mathrm{meq}$ of sodium bicarbonate and $200 \mathrm{mcg}$ of phenylephrine prior to intubation. She then had a generalized seizure followed by PEA arrest. Return of spontaneous circulation was achieved but she remained bradycardic and hypotensive with an immeasurably wide QRS interval. A total of 450 meq of sodium bicarbonate, two grams of magnesium, and $89 \mathrm{mg}$ of calcium were administered with an initial improvement in blood pressure and QRS to $172 \mathrm{~ms}$. The ECMO team was consulted, but elected not to intervene due to a brief improvement in the patient's blood pressure on maximal dose of norepinephrine, epinephrine, and vasopressin. The patient's hemodynamics and QRS duration worsened despite additional bicarbonate administration. Echocardiogram showed good biventricular contraction. Transcutaneous and transvenous pacing was attempted unsuccessfully. Methylene blue, Intralipid, and multiple doses of lidocaine were administered. The patient then had a second more prolonged PEA arrest. ROSC was obtained and the ECMO team cannulated the patient with normalization in hemodynamics.

Results: The patient was weaned off ECMO on hospital day three. EEG was without evidence of seizures. MRI showed focal areas of anoxic injury. The patient was discharged to a long-term care facility with minimal neurologic improvement.

Conclusion: ECMO may be a valuable tool in managing severe cyclic antidepressant overdose if available and considered early.

\section{Lithium Neurotoxicity: Silent but Deadly}

\author{
Raleigh D Ems ${ }^{1,2}$, Ryan T Marino ${ }^{1,2}$ \\ ${ }^{1}$ Unviersity Hospitals Cleveland Medical Center, Cleveland, Ohio, USA. \\ ${ }^{2}$ Case Western Reserve University School of Medicine, Cleveland, Ohio, \\ USA
}

Background: The Syndrome of Lithium-Effectuated Neurotoxicity (SILENT) is a rare, devastating complication of lithium therapy causing prolonged or permanent neurotoxicity and has been reported in patients without elevations in serum lithium concentration. SILENT can result in brainstem dysfunction, dementia and even death.

Hypothesis: SILENT may go unrecognized in patients with comorbid psychiatric illness and normal serum lithium levels.

Methods: This is a single-patient case report. A 70-year-old woman with a history of chronic kidney disease stage III, hypertension treated with lisinopril, and schizoaffective disorder treated with lithium, haloperidol, and quetiapine, presented to an outside hospital for two weeks of progressive confusion and lethargy after she reportedly self-discontinued her home medications.

Results: On initial examination, the patient was disheveled and speaking incoherently. Lithium level at presentation was $1.20 \mathrm{mmol} / \mathrm{L}$ (reference range $0.60-1.20$ ). Urine drug screen was negative for drugs of abuse. Her presentation was thought to be decompensated psychiatric illness and she was admitted psychiatrically with ziprasidone initiated in place of haloperidol and quetiapine and lithium continued for three days. Encephalopathy worsened and she was transferred to our tertiary care center on hospital day 12 . All medications were stopped. Serum lithium was undetectable at the time of toxicology consult on hospital day 14. Exam revealed inextinguishable ankle clonus persisting greater than 20 days after the last administration of a serotonergic agent, as well as ataxia, titubation, nystagmus, and upper extremity tremor. Extensive neurologic workup including serum, cerebral spinal fluid, brain magnetic resonance imaging, and electroencephalogram studies were negative for alternative causes of encephalopathy. Comprehensive inpatient results suggest diagnosis of SILENT. Despite supportive therapy, 24 days after presentation the patient became unresponsive, hypotensive, bradycardic, and died. Conclusion: Despite its high morbidity and mortality, SILENT may go unrecognized in the setting of comorbid psychiatric illness and can result in permanent neurologic dysfunction and death.

\section{Mortality from Lithium-Induced Cardiac Dysrhythmia}

\author{
Suresh K Pavuluri ${ }^{1,2}$, Rachel Shively ${ }^{1,2}$ \\ ${ }^{1}$ Elmhurst Hospital, NYC, NY, USA. ${ }^{2}$ The Mount Sinai Hospital, NYC, \\ $N Y$, USA
}

Background: Although lithium-induced cardiac dysrhythmias have been reported, there are few reports of mortality from lithium-induced ECG changes and ECG monitoring is not commonplace.

Methods: We present a case of mortality secondary to cardiac dysrhythmia in the setting of lithium toxicity.

Results: A 45-year-old male with bipolar disease on lithium, hypertension on lisinopril and furosemide, and diabetes presented to the ED with altered mental status progressive over three days. He had just started metformin and developed significant diarrhea. On arrival, he was unresponsive. He had ROSC after 10 minutes of ACLS including administration of calcium chloride and sodium bicarbonate. He was found to have acute renal failure (creatinine $9.21 \mathrm{mg} / \mathrm{dL}$ ), acidosis (bicarbonate 13 $\mathrm{mmol} / \mathrm{L}$ ), and hyperkalemia (potassium $6.5 \mathrm{mEq} / \mathrm{L}$ ), with sinoatrial bradycardia at $46 \mathrm{bpm}$ and prolonged QRS $(123 \mathrm{~ms})$ and QTc $(517 \mathrm{~ms})$. After treatment, his potassium and bicarbonate normalized $(3.6 \mathrm{mEq} / \mathrm{L}$ and $24 \mathrm{mmol} / \mathrm{L}$, respectively) but lithium was elevated at $3.31 \mathrm{mmol} / \mathrm{L}$. Despite this, his QRS prolonged to $191 \mathrm{~ms}$ and QTc to $619 \mathrm{~ms}$. He then developed a pulseless wide complex tachycardia. He received ACLS including amiodarone administration achieving ROSC and began sustained low-efficiency dialysis (SLED). Unfortunately, he soon developed pulseless wide complex bradycardia and could not be resuscitated. He had no recent ECG monitoring, with the last ECG taken four years prior showing sinus bradycardia with QRS $115 \mathrm{~ms}$.

Conclusion: Although this patient had hyperkalemia and acidosis in the setting of renal failure, his conduction abnormalities leading to fatal arrhythmia persisted after these were treated. Additionally, he showed prior evidence of conduction abnormality while on lithium but had no further ECG monitoring. Providers should consider ECG monitoring for patients on lithium, and lithium-induced conduction abnormalities when these patients present with arrhythmia. Finally, QTc prolonging agents such as amiodarone, should be used with caution.

\section{Severe Ibuprofen Toxicity Treated With Single-Pass Albumin Dialysis}

Sierra Foster, Kim Aldy

University of Texas Southwestern Medical Center, Dallas, Texas, USA

Background: Ibuprofen ingestion accounts for a large majority of intentional ingestions, especially in adolescents. Previous studies have shown that ibuprofen is $>90 \%$ protein bound and nondialyzable by conventional means.

Hypothesis: Does single-pass albumin dialysis (SPAD) increase clearance of ibuprofen from the blood and provide clinical improvement.

Methods: A 17-year-old girl presented an hour after reported ingestion of $2000 \mathrm{mg} / \mathrm{kg}$ of ibuprofen and two shots of vodka in a suicide attempt. Within four hours, she developed acidosis and exhibited lethargy. She required intubation for seizure-like activity and CNS depression. Metabolic acidosis, acute kidney injury, and electrolyte abnormalities worsened on hospital day (HD) one despite administration of sodium bicarbonate and CVVH initiation. She developed hypotension and was placed on infusions of norepinephrine, epinephrine, and vasopressin. SPAD containing $25 \%$ albumin was initiated on HD two and was performed daily for four days. Time matched quantitative ibuprofen levels from both serum and dialysate were obtained daily after SPAD. Serum ibuprofen concentration was $250 \mathrm{mcg} / \mathrm{mL}$ at 37 hours post ingestion. After the first SPAD session at 60 hours post ingestion, serum and dialysate ibuprofen concentrations were $21 \mathrm{mcg} / \mathrm{mL}$ and $22 \mathrm{mcg} / \mathrm{ml}$ respectively. Hypotension resolved after the first SPAD session and she was 
able to wean off all pressors. The patient's renal failure resolved and her mental status improved by HD five.

Results: Severe ibuprofen toxicity is rare, but large ingestions can lead to renal failure, acidosis, and death. Prior studies of ibuprofen clearance with hemodialysis have shown minimal to no dialysate recovery, likely due to high protein binding. Albumin dialysate allows for elimination of highly protein bound toxins. This patient ingested a fatal dose of ibuprofen and had multi-organ failure and shock that improved soon after SPAD was initiated.

Conclusion: SPAD may be a useful treatment in patients with massive ibuprofen overdose.

\section{Acidosis Managed with Hemodialysis Following Massive Ibuprofen Ingestion}

Sam T Ontiveros, Alicia B Minns, Priya Srihari, Richard F Clark University of California San Diego, San Diego, California, USA

Background: Ibuprofen ingestions typically cause minor gastrointestinal upset, but larger ingestions may cause renal injury, acidosis, and coma. Treatment with hemodialysis (HD) is not typically utilized due to ibuprofen's high protein binding.

Hypothesis: Hemodialysis may be utilized to treat the acidemia in severe ibuprofen poisonings.

Methods: This is a single-patient chart review. An 11-year-old 36.7-kg male presented with vomiting and obtundation five hours after ingesting four hundred $200 \mathrm{mg}$ tablets of ibuprofen. Labs showed a pH 7.23, pCO2 $43 \mathrm{mmHg}$, bicarbonate $15 \mathrm{mmol} / \mathrm{L}$, lactate $3.4 \mathrm{mmol} / \mathrm{L}$, and creatinine of $0.5 \mathrm{mg} / \mathrm{dL}$. Acetaminophen and salicylate levels were undetectable. The patient was intubated and flown to our children's hospital. No gastrointestinal decontamination was done. Repeat labs eight hours post-ingestion included $\mathrm{pH} 7.3$, and bicarbonate $21 \mathrm{mmol} / \mathrm{L}$. Intravenous hydration was continued overnight and the patient was extubated the following morning. However, his mental status later worsened and his serum $\mathrm{pH}$ declined to 6.9 , bicarbonate $10 \mathrm{mmol} / \mathrm{L}$, lactate $19 \mathrm{mmol} / \mathrm{L}$. The patient was reintubated, started on a bicarbonate infusion, and required norepinephrine and epinephrine infusions for hypotension. Hemodialysis was begun with a rapid improvement in hemodynamics. Vasopressors were discontinued, $\mathrm{pH}$ and bicarbonate normalized, and lactate downtrended. His renal function remained normal throughout his hospital stay. Serum ibuprofen level was $>360 \mathrm{mg} / \mathrm{L}$ (nl $10-50 \mathrm{mg} / \mathrm{L}$ ) prior to $\mathrm{HD}$ and declined to $213.5 \mathrm{mg} / \mathrm{L}$ after HD.

Results: The patient was extubated 24 hours later, but required high flow oxygen due to pulmonary edema and aspiration pneumonitis. He continued to clinically improve until discharge on hospital day 10 to an inpatient psychiatric unit.

Conclusion: Massive ibuprofen ingestion can cause coma, acidosis, pulmonary edema, and serum lactate elevation. Hemodialysis is an important supportive therapy, but efficacy of enhanced elimination remains unproven.

\section{A Critical Appraisal of CRRT Efficacy in Metformin Associated Lactic Acidosis}

Matthew S Correia ${ }^{1,2}$, B. Zane Horowitz ${ }^{3,4}$

${ }^{1}$ Oregon Health and Science University, Portland, OR, USA. ${ }^{2}$ Oregon Poison Center, Portland, OR, USA. ${ }^{3}$ Oregon Health and Science University, Portland, CA, USA. ${ }^{4}$ Oregon Poison Center, Portland, CA, USA

Background: Metformin Associated Lactic Acidosis (MALA) is a rare but potentially fatal condition related to the impairment of aerobic metabolism. Toxicity generally results from either the acute massive ingestion or from chronic toxicity related to impaired renal clearance. Although hemodialysis is the recommended extracorporeal treatment modality, many patients are too hemodynamically unstable to tolerate large fluid shifts. Continuous renal replacement therapy (CRRT) is considered an alternative modality but high-quality pharmacokinetic data is not available.

Methods: We present a case of a 57-year-old male with underlying cirrhosis, ongoing alcohol abuse, and non-insulin dependent diabetes mellitus who experienced MALA and profound hemodynamic collapse in the setting of acute renal failure after initiation of metformin for hyperglycemic control one month prior. On presentation: $\mathrm{pH}$ was 6.80 , bicarbonate $5 \mathrm{mmol} / \mathrm{L}$, anion gap was 40, lactic acid $32 \mathrm{mmol} / \mathrm{L}$, Cr $1.6 \mathrm{mg} /$ $\mathrm{dL}$, beta hydroxybutyrate $11.7 \mathrm{mg} / \mathrm{dL}$, and osmolar gap $58 \mathrm{mOsm} / \mathrm{kg}$. Fomepizole was administered due to concern for toxic alcohol ingestion; however, methanol and ethylene glycol returned undetectable. He was started on intravenous bicarbonate and CRRT was initiated at a rate of 30 $\mathrm{ml} / \mathrm{kg} /$ hour. He weighed $71.2 \mathrm{~kg}$. Inflow and outflow metformin concentrations were $5.3 \mathrm{mg} / \mathrm{dL}$ and $4.3 \mathrm{mg} / \mathrm{dL}$ (ref $1-2 \mathrm{mg} / \mathrm{dL}$ ), respectively, resulting in an instantaneous extraction ratio of $18 \%$ and extracorporeal clearance rate of $6.72 \mathrm{~mL} / \mathrm{min}$. Despite aggressive resuscitative efforts, he remained persistently acidotic and was transitioned to comfort measures the following morning.

Conclusion: In our patient with profound MALA with hemodynamic instability, CRRT had limited efficacy in the extracorporeal clearance of metformin. Intermittent hemodialysis should be pursued as the modality of choice pending further CRRT pharmacokinetic clearance data.

055. Severe Lactic Acidosis and Hemodynamic Instability in Combined Metformin and Amlodipine Overdose Treated With Continuous Renal Replacement Therapy (CRRT)

Michael Yeh ${ }^{1,2}$, Jared Cavanaugh ${ }^{3}$, Matthew D Eisenstat ${ }^{1,2}$, Colin Therriault $^{1,2}$, Brent W Morgan ${ }^{1,2}$

${ }^{1}$ Emory University, Atlanta, GA, USA. ${ }^{2}$ Georgia Poison Center, Atlanta, GA, USA. ${ }^{3}$ California Northstate University, Sacramento, CA, USA

Background: Metformin, a biguanide used to treat diabetes mellitus, is associated with profound metabolic acidosis with elevated lactate in overdose. Amlodipine, a dihydropyridine calcium channel antagonist, lowers blood pressure by acting on vascular smooth muscle to decrease peripheral vascular resistance. Combined, these agents could result in severe metabolic acidosis, hypothermia, hypoglycemia, and hemodynamic instability.

Methods: A 15-year-old female presented comatose, bradycardic, and hypotensive after an unknown overdose. She had access to metformin, amlodipine, aspirin, meloxicam, and naproxen. Vitals showed heart rate 50, temperature $32.7^{\circ}$ Celsius, blood pressure $109 / 76$, respiratory rate 15 . Results: The patient's condition worsened, with heart rate of $40 \mathrm{~s}$, and blood pressure of 90/30. Labs showed pH 6.5, pCO2 47, bicarbonate $<5$, glucose 37, anion gap 42.9, lactate $>20 \mathrm{mmol} / \mathrm{L}$, creatinine 2.24 , INR 4.3, and undetectable acetaminophen, salicylates, and ethanol. She received atropine and epinephrine with transient improvement. She had three generalized seizures and received dextrose and lorazepam. She required intubation and pressor support with epinephrine and vasopressin. Additional therapies included glucagon, calcium gluconate, and a sodium bicarbonate infusion. Due to persistent acidemia, elevated lactate, and concern for metformin ingestion, CRRT was instituted. Her hemodynamics, renal function, and coagulopathy stabilized. She was extubated on hospital day four. Pressors were discontinued on hospital day five and mental status returned to baseline. Initial serum samples revealed a metformin level of $45 \mathrm{mcg} / \mathrm{mL}$ (therapeutic range $1-2 \mathrm{mcg} / \mathrm{mL}$ ) and amlodipine level of $97 \mathrm{ng} / \mathrm{mL}$ (therapeutic range $2-25 \mathrm{ng} / \mathrm{mL}$ ).

Conclusion: Metformin overdose may result in elevated lactate, severe metabolic acidosis, and shock. Amlodipine co-ingestion likely exacerbated this patient's peripheral vasodilation and shock. NSAIDs may have also contributed to acidosis and hypotension. Correction of acidemia with 
CRRT potentially improved responsiveness to catecholamines, resulting in restored vascular tone and resolution of vasodilatory shock.

\section{Sustained Release Potassium Overdose Requiring Prolonged Hemodialysis}

Lesley C Pepin ${ }^{1}$, Nik Matsler ${ }^{1}$, Alexa Camarena-Michel ${ }^{2}$, Matthew Zuckerman $^{3,2}$

${ }^{1}$ Denver Health Emergency Medicine Residency, Denver, CO, USA.

${ }^{2}$ Rocky Mountain Poison and Drug Safety, Denver, CO, USA.

${ }^{3}$ University of Colorado Hospital Department of Emergency Medicine, Aurora, CO, USA

Background: Life-threatening hyperkalemia from oral potassium overdose is rare. Massive overdose of sustained-release products can overwhelm intrinsic potassium excretion necessitating invasive management. Hypothesis: Massive extended release potassium overdose may require hemodialysis despite normal renal function.

Methods: This is a single-patient chart review. A 72-year-old woman with a history of hypokalemia presented with an intentional ingestion of $1800 \mathrm{mEq}$ of sustained release potassium chloride tablets. The patient arrived hemodynamically stable one hour after ingestion. Initial workup revealed: normal electrocardiogram, potassium $5.7 \mathrm{mmol} / \mathrm{L}$, creatinine $0.65 \mathrm{mg} / \mathrm{dL}$, ethanol $158 \mathrm{mg} / \mathrm{dL}$, negative acetaminophen and salicylate concentrations, and negative urine drug screen. Abdominal radiograph did not demonstrate gastric radiopaque material. The patient was given calcium gluconate, regular insulin, albuterol, furosemide, and calcium polystyrene. Whole bowel irrigation was not performed because of pillinduced ileus.

Results: Potassium peaked at $9.6 \mathrm{mmol} / \mathrm{L}$ seven hours post-ingestion (creatinine $0.75 \mathrm{mg} / \mathrm{dL}$ ). The patient received hemodialysis for four hours for refractory hyperkalemia. Post-dialysis potassium rose from 6.2 to 9.2 $\mathrm{mmol} / \mathrm{L}$ over five hours despite normal creatinine $(0.67 \mathrm{mg} / \mathrm{dL})$. Hemodynamic instability with a sinusoidal wave pattern on electrocardiogram evolved. The patient required calcium chloride, regular insulin, sodium bicarbonate, and albuterol to restore hemodynamic stability. The patient underwent six additional hours of hemodialysis. Due to persistent post-dialysis hyperkalemia $(5.1-6.1 \mathrm{mmol} / \mathrm{L})$, continuous renal replacement therapy was pursued for eight additional hours with normalization of potassium to $4 \mathrm{mmol} / \mathrm{L}$. The patient was medically cleared four days after ingestion.

Conclusion: Massive overdoses of sustained-release potassium may not respond to pharmacologic interventions, even in cases of normal renal function. Ileus formation may prolong absorption of potassium, overwhelming intrinsic potassium excretion. Early, prolonged hemodialysis may be indicated for medication-induced hyperkalemia.

\section{Intentional Caffeine Overdose Requiring Hemodialysis}

Lawrence Liao, Bryan Judge, Brad Riley, Jeffrey S Jones

Spectrum Health - Michigan State University Emergency Medicine Residency Program, Grand Rapids, MI, USA

Background: Caffeine overdose can cause profound hypokalemia, lactic acidosis, seizures, hemodynamic instability, cardiac dysrhythmias, and rhabdomyolysis. Hemodialysis should be considered in cases of severe toxicity due to caffeine's small volume of distribution, low molecular size, and minimal protein binding.

Methods: Single-patient chart review. We present the case of a young man who presented after intentional large ingestion of caffeine pills and subsequently needing hemodialysis. A 35-year-old male presents to an outlying hospital with altered mental status and supraventricular tachycardia (SVT) after intentional caffeine ingestion of ninety caffeine pills for a total of $18 \mathrm{gm}$ of caffeine (lethal dose approximately $15 \mathrm{gm}$ ). He was given activated charcoal and transferred to the intensive care unit at a tertiary care facility. On arrival, patient had marked tachycardia, diaphoresis, agitation but otherwise hemodynamically stable. He was intubated shortly after arrival secondary to hypoxia from aspiration of activated charcoal during transport. Laboratory studies were remarkable for lactic acid $4.1 \mathrm{mEq} / \mathrm{L}$, hypokalemia of $2.5 \mathrm{mEq} / \mathrm{L}, \mathrm{pH} 7.18$, and bicarbonate $15.8 \mathrm{mEq} / \mathrm{L}$. Nephrology recommended intermittent hemodialysis for 12 hours. CPK reached a peak of $1,895 \mathrm{U} / \mathrm{L}$. SVT resolved after hemodialysis and no episodes of seizures were documented while hospitalized. Patient extubated and discharged to inpatient psychiatric hospital. Conclusion: Due to the low number of reported cases, there is no consensus on the standard of care for treatment of severe caffeine overdose. Toxic levels can be reached quickly after ingestion and last for prolonged periods of time secondary to caffein's 3- to 10-hour half-life. This case details an adult male who presented to the emergency department after ingesting a potentially lethal caffeine. After treatment with intermittent hemodialysis, the patient's caffeine level decreased dramatically with resolution of tachyarrhythmias and metabolic derangements.

\section{Unintentional Caffeine Overdose with Severe Toxicity}

Joshua R Codding, Hugo Hanson III, Laura J Fil

Vassar Brothers Medical Center, Poughkeepsie, NY, USA

Background: Caffeine is the most widely used and socially acceptable stimulant drug in the United States. The public is generally unaware of the potential dangers of caffeine overuse as warnings about overuse are not widespread.

Method: This is a single-patient chart review. A 21-year-old male with a past medical history of bipolar disorder and substance abuse presented non-responsive, status-post seizure in respiratory distress. He was emergently intubated. Initial vital signs were: BP: 186/82 mmHg; HR: 98/min; RR: 20/min; O 2 sat: $85 \%$ RA; T: 98.0 o F. The patient's exam was significant for: a non-responsive patient with sonorous respirations and extreme diaphoresis. Pupils were dilated and reactive. Lung and heart exam were normal. History obtained from the family revealed the patient has been using a pre-workout powder called "Woke AF" purchased at a national retail store. They reported the patient would often use multiple scoops in one drink. The caffeine dose of one scoop is $333 \mathrm{mg}$. A typical cup of coffee is 40-130 mg.

Results: Labs were significant for: an anion gap metabolic acidosis and $\mathrm{pH}$ of 6.85. Lactate level of 21.5. Head CT was normal. Chest CT showed evidence of aspiration, bilateral small pneumothorax, and pneumomediastinum. Caffeine level was drawn 10 hours after arrival resulting in $1.2 \mathrm{mg} / \mathrm{L}$. Caffeine is a methylxanthine like theophylline; in fact, $5 \%$ of caffeine metabolites become theophylline endogenously. Seizures are likely caused by GABA antagonism and lowering seizure threshold with A1 antagonism and release of excitatory neurotransmitters. There is no antidote, making the standard of care supportive.

Conclusion: Severe caffeine toxicity can be a dangerous and non-specific presentation in the emergency room and can occur from FDA-approved products, highlighting the need for stricter regulation or increased public education and warning labels focusing on the dangers of excessive caffeine use.

\section{Survey of Poison Center Approaches to Bupropion Ingestion}

Ron Kirschner ${ }^{1,2}$, Karen Smith ${ }^{2}$, Marcia Rasmussen ${ }^{2}$

${ }^{I}$ University of Nebraska Medical Center, Omaha, NE, USA. ${ }^{2}$ Nebraska Regional Poison Center, Omaha, NE, USA

Background: Bupropion ingestion is associated with higher rates of seizure and greater morbidity compared to other antidepressants. With 
widespread use of extended release formulations, delayed seizures are a concern; optimal observation time for asymptomatic patients is uncertain. Methods: This is an IRB-approved survey study of poison center medical, managing, and associate directors to assess variability of recommendations following bupropion ingestion. An electronic survey link was sent through the American Association of Poison Control Centers' director listserv $(n=232)$. The survey asked about threshold amounts for health care facility (HCF) referral following pediatric exploratory ingestion (PEI) and adult unintentional ingestion (AUI), recommendations for activated charcoal (AC), and observation time for asymptomatic patients. Results: There were 33 responses (14.2\% response rate). Regarding PEI, a majority $(57.6 \%)$ used a $>10 \mathrm{mg} / \mathrm{kg}$ threshold for HCF referral with immediate release (IR) formulations. For sustained/extended release (SR/ $\mathrm{XL}$ ) formulations, $42.4 \%$ used $>10 \mathrm{mg} / \mathrm{kg}$ for $\mathrm{HCF}$ referral and $27.3 \%$ would refer any amount. Most common referral thresholds for AUI were $450 \mathrm{mg}(33.3 \%)$ and $600 \mathrm{mg}(30 \%)$. Most (87.9\%) indicated they would recommend $\mathrm{AC}$ for adult intentional $\mathrm{SR} / \mathrm{XL}$ ingestions; fewer would do so in PEI $(75.8 \%$ and $54.6 \%$ for unknown amounts of SR/XL and IR formulations, respectively). A minority (6.1\%) do not recommend AC for bupropion overdose. Regarding observation periods after PEI, most recommend 6-12 hours for IR (82.8\%) and 16-24 hours for SR/XL (84.4\%). For AUI, $74.1 \%$ recommend $4-12$ hours observation. For intentional overdose, $75.9 \%$ recommend $4-12$ hours observation with IR formulations; $93.8 \%$ recommend 16-24 hours with SR/XL. Some commented resolution of symptoms was a key determinant of disposition.

Conclusion: The study was limited by low response rate. Delayed seizures following bupropion ingestion are well recognized. Recommendations of poison center toxicologists regarding observation times and use of AC remain variable.

\section{Acute Encephalopathy in the Setting of Toxic Clobazam (Onfi) Metabolite Levels and the Unassuming Antidote}

Breanna K Bates ${ }^{1}$, John L Fletcher ${ }^{2}$, Trager Hintze ${ }^{3}$, Shaheyar J Hafeez ${ }^{4}$, Ali Seifi ${ }^{4}$, Colleen Barthol, $\mathrm{P}^{3}$, Shawn M Varney ${ }^{1,5}$, Han T Gao ${ }^{1,5}$

${ }^{1}$ UT Health Department of Emergency Medicine, San Antonio, Texas, USA. ${ }^{2}$ Division of Trauma and Critical Care, Brooke Army Medical Center, San Antonio, Texas, USA. ${ }^{3}$ Department of Pharmacotherapy \& Pharmacy Services, Neurocritical Care, San Antonio, Texas, USA. ${ }^{4}$ Department of Neurosurgery, Division of Neurocritical Care, San Antonio, Texas, USA. ${ }^{5}$ South Texas Poison Center, San Antonio, Texas, USA

Background: Clobazam is a 1,5-benzodiazepine anticonvulsant. We describe a patient with undifferentiated acute encephalopathy who had an unmeasurably high $\mathrm{N}$-desmethylclobazam level reversed by a flumazenil infusion.

Methods: This is a single-patient chart review. A 48-year-old female with history of West Nile encephalitis-induced epilepsy and refractory epilepsy presented to an outside emergency department with altered mental status (AMS) which developed over two days and hyponatremia. Her medications included oxcarbazepine, levetiracetam, topiramate, and clobazam. Her AMS persisted despite improved serum sodium levels. EEG showed bihemispheric epileptogenicity associated with cortical dysfunction. When transferred to our ICU, she was somnolent with a Glasgow Coma Scale (GCS) score of 8 (eyes-2, verbal-2, motor-4), had spastic quadriparesis with sustained bilateral lower extremity clonus, opthalamoplegia, hypotension, and decreased respirations. Multiple tests were ordered including autoimmune, bacterial/fungal cultures, cerebral spinal fluid, meningitis/encephalitis polymerase chain reaction, autoimmune encephalitis panel, and antiepileptic drug levels. Labs were remarkable only for CRP $222 \mathrm{mg} / \mathrm{L}$, serum albumin $1.2 \mathrm{~g} / \mathrm{dL}$, normal clobazam level, and N-desmethylclobazam >10,000 ng/mL (normal 300-3,000 ng/ $\mathrm{mL}$ ). Clobazam was discontinued and albumin was given. After five days of unchanged AMS, she was given a flumazenil bolus and infusion. One hour later her GCS improved from 2T4 to 4T6, and the following day she was extubated. One day later, her flumazenil infusion was inadvertently stopped for four hours. Her mental status deteriorated but improved once flumazenil was restarted. After five days of flumazenil infusion, she had a GCS of 14, oriented to self. We expect the patient will require flumazenil infusion for another week given the 7-9-day half-life of $\mathrm{N}$ desmethylclobazam and the last level still measuring $>10,000 \mathrm{ng} / \mathrm{mL}$. Continuous EEG did not demonstrate seizure recurrence on flumazenil. Conclusion: As a benzodiazepine antidote, flumazenil can reverse the effects of clobazam metabolite toxicity resulting in improved encephalopathy without rebound seizures.

\section{Use of Meropenem for Enhanced Elimination in the Setting of} Valproic Acid Overdose: a Case Report

Jaclyn M Stoffel ${ }^{1}$, Kelsey Kohman ${ }^{1}$, Joshua R McFalls ${ }^{2,3}$, Dazhe James $\mathrm{Cao}^{3}$

${ }^{1}$ Baylor University Medical Center, Dallas, TX, USA. ${ }^{2}$ Parkland Memorial Hospital, Dallas, TX, USA. ${ }^{3}$ University of Texas Southwestern Medical Center, Dallas, TX, USA

Background: Valproic acid (VPA) is an anti-epileptic medication that has many known drug-drug interactions. Meropenem and other carbapenems have been proposed to increase the elimination rate of VPA in patients who overdose.

Hypothesis: Meropenem can be administered to patients as an adjunctive treatment to increase VPA elimination in overdose presentation.

Methods: This is a single case chart review. A 20-year-old patient with no past medical history arrived at the emergency department 8.5 hours after an intentional ingestion of 30 tablets of valproic acid that was not prescribed to her. On initial presentation, she had stable vitals and no evidence of altered mental status. Laboratory workup showed an initial VPA level of $>300 \mathrm{mcg} / \mathrm{mL}$. She had normal renal and hepatic function. She was admitted to the telemetry for observation and trending of VPA levels. Overnight, repeat VPA levels were noted to be $>300 \mathrm{mcg} / \mathrm{mL}$. Fourteen hours after reported ingestion, the patient was started on a carbapenem with a goal of enhancing the elimination of VPA.

Results: The patient was started on $500 \mathrm{mg}$ of IV meropenem every 8 hours. Two hours after the first dose of meropenem, repeat VPA level was $239.4 \mathrm{mcg} / \mathrm{mL}$. VPA levels continued to down trend with the next labs showing $90.2 \mathrm{mcg} / \mathrm{mL}, 63.2 \mathrm{mcg} / \mathrm{mL}$, and $27.3 \mathrm{mcg} / \mathrm{mL}$ at 22,24 , and 28 hours post ingestion respectfully. This represents an apparent half-life of 3-4 hours. This apparent half-life is much lower than the reported VPA half-life of 9-16 hours in the VPA package insert.

Conclusion: Although this drug interaction is well described, the intentional use of meropenem to enhance VPA excretion is not well described in the literature. This case represents the successful use of meropenem to enhance the elimination of VPA.

062. An Uncommon Cause of High-Anion Gap Metabolic Acidosis After Repeated Supratherapeutic Paracetamol Ingestion

Hwee Min D Lee ${ }^{1}$, Yit Hung Leang ${ }^{2}$, Andis Graudins ${ }^{1}$

${ }^{1}$ Monash Health, Melbourne, Victoria, Australia. ${ }^{2}$ Austin Health, Melbourne, Victoria, Australia

Background: High-anion gap metabolic acidosis (HAGMA) commonly results from excess urea, lactate, ketones, and toxin exposure (e.g., salicylate, toxic alcohols). Accumulation of pyroglutamic acid (PGA) is a rare cause of HAGMA. We describe a case of confirmed PGA accumulation after excessive paracetamol use.

Methods: A 32-year-old female presented with shingles pain despite having taken $96 \times 665 \mathrm{mg}$ modified-release paracetamol over the preceding two days. Two years prior, excessive ibuprofen use for dysmenorrhea 
caused a gastric ulcer perforation. She subsequently substituted ibuprofen with four paracetamol every 3-4 hours regularly. On presentation, vital signs were normal except respiratory rate $24 / \mathrm{min}$. Venous blood gas: $\mathrm{pH}$ 7.04, bicarbonate $5.0 \mathrm{mmol} / \mathrm{L}, \mathrm{pCO} 219 \mathrm{mmHg}$, lactate $1.4 \mathrm{mmol} / \mathrm{L}$, sodium $150 \mathrm{mmol} / \mathrm{L}$, chloride $117 \mathrm{mmol} / \mathrm{L}$ and anion gap 28 (HAGMA). Serum ketones $2.8 \mathrm{mmol} / \mathrm{L}$, salicylate undetected and normal osmolar gap. Serum paracetamol was $312 \mathrm{micromol} / \mathrm{L}, 2.5$ hours after the last dose. Liver function, INR and creatinine were normal. PGA accumulation from chronic paracetamol misuse and glutathione depletion was suspected. Urine was sent for PGA assay. Treatment included 20 hours of acetylcysteine infusion to replenish glutathione, intravenous crystalloids and sodium bicarbonate infusion. HAGMA resolved over 36 hours. Serum ALT and INR remained normal. Qualitative urine assay was positive for PGA.

Conclusion: The exact incidence of HAGMA caused by PGA accumulation is unknown. PGA is processed by the glutamate-glutathione cycle. The commonest cause of PGA accumulation is glutathione depletion. This causes the loss of negative feedback on gamma-glutamylcysteine (PGA precursor) production. In our case, chronic excessive paracetamol use and a staggered overdose caused glutathione depletion. Other causes include malnutrition, alcoholism, and liver failure. Though rare, deficiencies of enzymes in the glutamate-glutathione cycle can also cause PGA accumulation. Treatment includes ceasing paracetamol and glutathione replenishment with acetylcysteine. PGA accumulation should be considered in the differential diagnosis of unexplained HAGMA, especially with a history of excessive supratherapeutic paracetamol use.

\section{Rare Case of Massive Acetaminophen Poisoning Resulting in Methemoglobinemia}

Marielle Brenner ${ }^{1,2}$, Robert Avera ${ }^{1,2}$, Jennifer Acciani ${ }^{1,2}$

${ }^{1}$ Indiana University School of Medicine, Indianapolis, IN, USA. ${ }^{2}$ Indiana

Poison Center, Indianapolis, IN, USA

Background: Methemoglobinemia was a common adverse effect of the antipyretics phenacetin and acetanilide. Their metabolite, acetaminophen, replaced them in 1950, as it was believed to not cause methemoglobinemia. Since then, there have been only a handful of cases of methemoglobinemia associated with acetaminophen ingestions, all of which have been treated with methylene blue.

Methods: This is a single-patient chart review. 38-year-old man admitted to the hospital two days after a reported single ingestion of $100 \mathrm{~g}$ of acetaminophen in a suicide attempt. He had no other medications. Presenting vital signs included oxygen saturation $79 \%$ on room air that was unresponsive to supplemental oxygen and respiratory rate 30 breaths per minute. On exam, he was tachypneic but not cyanotic. ECG demonstrated normal sinus rhythm with prolonged QTc $517 \mathrm{msec}$. Chest X-ray was unremarkable and he tested negative for COVID-19. His 56-hour acetaminophen concentration was $25.3 \mathrm{mcg} / \mathrm{ml}$. Presenting laboratory values included AST $6580 \mathrm{U} / \mathrm{L}$, ALT $8100 \mathrm{U} / \mathrm{L}$, INR 1.93, hemoglobin $13.4 \mathrm{~g} / \mathrm{dL}$, methemoglobin concentration of $12.4 \%$, and sulfhemoglobin concentration $0.6 \%$. Toxicology was consulted and recommended Nacetylcysteine (NAC) therapy and did not recommend methylene blue as the patient denied any dyspnea or cyanosis.

Results: The patient received ascorbic acid $1.5 \mathrm{~g}$ every six hours for four doses for the methemoglobinemia. Testing for glucose-6-Pdehydrogenase deficiency was negative. Further methemoglobin concentrations could not be obtained due to turbidity in repeat samples. He improved clinically on hospital day three when his oxygen saturation improved to $95 \%$ on room air with respiratory rate 22 breaths per minute. NAC was discontinued on hospital day four. He was discharged on hospital day 15 with normal transaminase concentrations and INR.

Conclusion: While rare, methemoglobinemia can occur with isolated acetaminophen poisoning but does not necessitate methylene blue treatment if typical criteria for methemoglobin treatment have not been met.
064. Profound Hypertriglyceridemia Due to Intravenous Lipid Emulsion Administration Error Treated with Lipopheresis

Kevin Baumgartner, Michael Mullins, Evan Schwarz

Washington University School of Medicine, Saint Louis, MO, USA

Background: Intravenous lipid emulsion (ILE) is used in the treatment of life-threatening poisoning but excess amounts, including from supratherapeutic dosing, are hazardous.

Methods: This is a case report. A 65-year-old woman presented to a community hospital after ingesting an unknown amount of verapamil, atenolol, and olmesartan. She developed bradycardia and shock and was treated with crystalloids, glucagon, calcium, epinephrine, norepinephrine, and vasopressin. Her condition worsened and the state Poison Control Center (PCC) recommended hyperinsulinemic-euglycemic therapy (HIET), which was initiated. Hypotension persisted and the PCC recommended treatment with ILE. A $1.5 \mathrm{~mL} / \mathrm{kg}$ bolus of ILE was administered, followed by an infusion of $0.25 \mathrm{~mL} / \mathrm{kg} / \mathrm{min}$. The patient was then transferred for consideration of veno-arterial extracorporeal membrane oxygenation (VA ECMO). The ILE infusion was inadvertently continued at a rate of $0.25 \mathrm{~mL} / \mathrm{kg} / \mathrm{min}$ for approximately seven hours, until her arrival at the receiving hospital. She received a total of approximately 7.7 liters of ILE.

Results: The ILE infusion was discontinued and she was cannulated for VA ECMO. Her blood was noted to be grossly lipemic with a triglyceride concentration of $9,494 \mathrm{mg} / \mathrm{dL}$. The medical team including Toxicology elected to proceed with lipopheresis; this was performed in order to attempt to prevent clogging of the ECMO circuit, ARDS, and interference with critical laboratory diagnostics. A single session of lipopheresis was performed; one plasma volume was exchanged, with albumin and plasma as the replacement fluid. Triglycerides fell to $186 \mathrm{mg} / \mathrm{dL}$. Unfortunately, although the patient's cardiac function recovered, she had a prolonged ICU course complicated by ARDS and septic shock, and care was withdrawn on hospital day 14 .

Conclusion: Administration errors are dangerous adverse drug events. Early lipopheresis after ILE overdose can correct gross lipemia and normalize triglycerides but may not avoid associated morbidity and mortality.

065. Fatal Overdose Involving Intravenous Lipid Emulsion Supplement: a Case Report

George P Warpinski

Department of Medical Toxicology, Banner University Medical Center, Phoenix, Arizona, USA

Background: To meet their full nutritional requirements, patients receiving parenteral nutrition require lipid supplementation. Cases of intravenous lipid emulsion (IVLE) overdose treated with exchange transfusion have been described; however, cases of fatal IVLE overdose involving delay to exchange transfusion have not been.

Hypothesis: Rapid administration of IVLE is associated with metabolic acidosis, DIC, ARDS, with delay to exchange transfusion associated with worse outcomes.

Methods: This is a single-patient chart review. A nine-month-old infant dependent on parenteral nutrition presented after developing respiratory distress, epistaxis and a single episode of hematemesis at home. Several hours earlier at home the patient's $250 \mathrm{~mL}$ lipid supplement had been administered over the course of approximately four hours instead of 20 hours due to an administration error involving the infusion pump.

Results: The patient was admitted to the pediatric ICU. Significant lab findings on admission included: venous $\mathrm{pH} 7.15$, lactic acid $7.2 \mathrm{mEq} / \mathrm{L}$, PT $32.0 \mathrm{sec}$, aPTT $76.3 \mathrm{sec}$, fibrinogen $157 \mathrm{mg} / \mathrm{dL}$, platelets $51 \mathrm{~K} / \mathrm{uL}$, triglycerides $>1100 \mathrm{mg} / \mathrm{dL}$ (normal $30-150 \mathrm{mg} / \mathrm{dL}$ ). During their hospital course, the patient developed worsening lactic acidosis to $\mathrm{pH}$ of 6.92 , 
lactic acid to $17 \mathrm{mEq} / \mathrm{L}$, leading to asystole. After ROSC was achieved, exchange transfusion was performed. Although the patient had improvement of their triglyceride level to $390 \mathrm{mg} / \mathrm{dL}$ following exchange transfusion with $975 \mathrm{~mL}$ removed and $900 \mathrm{~mL}$ added the child developed hypotension refractory to vasopressors. Towards the end of the first hospital day, the patient developed ARDS and bleeding around the endotracheal tube. The patient demonstrated poor neurologic function post cardiac arrest by the end of the first day. The decision was made to withdraw care after discussion with the patient's caregiver. Death was pronounced shortly after.

Conclusion: Overdoses involving intravenous lipid emulsion can be fatal, especially if exchange transfusion is delayed.

\section{EMLA Toxicity in Preparation for Pulsed Dye Laser Therapy}

Jonathan R Eisenberg ${ }^{1}$, Natalie J Tedford ${ }^{1}$, Sarah A Becker ${ }^{1}$, Nicholas Weaver ${ }^{1}$, Michael J Moss ${ }^{1,2}$

${ }^{1}$ University of Utah, Salt Lake City, UT, USA. ${ }^{2}$ Utah Poison Control Center, Salt Lake City, UT, USA

Background: EMLA is a eutectic mixture of lidocaine and prilocaine used as a topical anesthetic for painful superficial procedures. Toxicity is uncommonly reported with its use.

Method: This is a single-patient chart review. A 5-month-old female with a segmental port wine stain presented 1.5 hours post application of EMLA (lidocaine/prilocaine) prior to scheduled pulse dye laser therapy with subsequent altered mental status, disordered breathing, and abnormal movements. On presentation to the ED, the patient appeared to be in status epilepticus that was refractory to benzodiazepine and levetiracetam therapy. Administration of propofol and rocuronium with intubation was able to control seizures. EKG did not demonstrate cardiotoxicity. Approximately two hours after EMLA application, lidocaine level was $5.2 \mathrm{mcg} / \mathrm{mL}$ and methemoglobinemia level was $5.7 \%$

Results: Recommended maximum total dose for age 3-12 months is up to two $\mathrm{g}$ with no more than $20 \mathrm{~cm}^{2}$ for application area. In our patient, $15 \mathrm{~g}$ of $2.5 \%$ lidocaine/prilocaine was $375 \mathrm{mg}$ of each drug. Her initial lidocaine level was $5.2 \mathrm{mcg} / \mathrm{mL}$ or roughly 43 times the peak serum concentration advised or two times the toxic level of $2.4 \mathrm{mcg} / \mathrm{mL}$. Assuming peak concentration of $5.2 \mathrm{mcg} / \mathrm{ml}$ and volume of distribution of $1.5 \mathrm{~L} / \mathrm{kg}$, weight of $7.92 \mathrm{~kg}$, she may have absorbed $61 \mathrm{mg}$ of drug or $16 \%$ of the applied dose. If her port wine stain represents $27 \%$ of her 0.38 $\mathrm{m}^{2} \mathrm{BSA}$ (arm and torso), this is an area of about $1000 \mathrm{~cm}^{2}$ or 50 times the recommended maximum application area. She was extubated nine hours after administration of EMLA. EEG showed no discrete epileptiform activity but absence of age expected background rhythms.

Conclusion: Topical anesthetic application in infants should be carefully measured and monitored to avoid adverse outcomes. Neurotoxicity with seizures can be controlled with conventional abortive methods but may require multiple lines of therapy, airway management, and lipid emulsion therapy if refractory.

\section{Recurrent Sinus Arrest After Local Intraoral Bupivacaine Injection}

Priya Srihari, Alicia B Minns, Bryan Corbett UCSD, San Diego, CA, USA

Background: Systemic toxicity from local anesthetics after dental procedures is rare, and can manifest as cardiac and/or neurologic events. Recurrent episodes that resolve without intervention are not typically reported.

Hypothesis: Recurrent intermittent sinus arrest can occur after a large intraoral injection of bupivacaine, possibly due to local toxicity in close proximity to the vagus nerve.
Methods: A 15-year-old male underwent routine tooth extraction. He received $14 \mathrm{~mL}$ of $0.25 \%$ bupivacaine with epinephrine and $10 \mathrm{~mL}$ of $1.3 \%$ liposomal bupivacaine intraorally. One hour after bupivacaine administration, he experienced an episode of bradycardia followed by 10 seconds of asystole. This occurred an additional five times over the next hour. During each episode, the patient became asystolic without a palpable pulse, unresponsive, each lasting approximately 10 to 20 seconds. The patient would become briefly hypertensive with mydriasis and would return to his baseline mental status between episodes. Echocardiogram was performed the subsequent day and was normal. An outpatient seven-day cardiac monitor demonstrated episodes of sinus bradycardia and sinus pauses lasting less than 10 seconds each; however, the patient remained asymptomatic.

Results: Venous blood gas obtained two and a half hours after the initial event showed a $\mathrm{pH}$ of 7.314 and a pCO2 of $50.7 \mathrm{~mm} \mathrm{Hg}$. A lactic acid level was $3.0 \mathrm{mmol} / \mathrm{L}$ (normal $<2.1 \mathrm{mmol} / \mathrm{L}$ ). Troponin, total creatine kinase, brain natriuretic peptide, and serum electrolytes were all within normal limits. Electrocardiogram showed normal sinus rhythm with sinus arrhythmia. Echocardiogram was unremarkable with an ejection fraction of $71 \%$. Bupivacaine level obtained 6 hours after the intraoral injection was $0.20 \mathrm{mcg} / \mathrm{mL}$.

Conclusion: Bupivacaine levels were not significantly elevated, suggesting that systemic toxicity was not likely the cause of patient's recurrent episodes. The recurrent nature of his episodes may have been a result of local toxicity near the vagus nerve.

\section{Second Degree Burns and Systemic Toxicity After Topical Cantharidin Use}

Vincent Calleo, Jeanna Marraffa, Christine Stork SUNY Upstate Medical University, Syracuse, NY, USA

Background: Topical cantharidin is used by many dermatologists to treat molluscum contagiosum. Significant systemic toxicity is reported after ingestion; however, topical adverse effects are limited to dermal irritation and blistering at the application site. We hypothesize systemic cantharidin toxicity can occur after topical cantharidin use.

Methods: This is a chart review from a single-patient encounter. A healthy two-year-old female with molluscum contagiosum on her torso and upper extremities was treated using $0.7 \%$ cantharidin topical cream by her dermatologist. No coverings were placed on the application sites, and the patient was fully dressed in long sleeves after application. Shortly later, the patient became irritable. Two hours after application, the child was bathed. The following morning, blisters formed at the application sites and the patient had visible blood in her urine. A urine specimen was obtained by her pediatrician which showed gross hematuria and the patient was referred to the emergency department for further care. Physical examination revealed a $12 \%$ body surface area second degree burns on her torso and upper extremities. Laboratory testing, including a complete blood count, comprehensive metabolic panel, and creatine kinase, was within normal limits. The patient was hospitalized and required multiple wound debridements. Her hematuria resolved three days after exposure and a repeat basic metabolic panel five days after exposure was normal. Results: There is a paucity of literature illustrating systemic involvement after topical cantharidin application. This case is unique in that hematuria developed after dermal use. Despite developing hematuria and significant second degree burns, the patient did well with supportive management.

Conclusion: Though uncommon, topical cantharidin use can cause systemic involvement as well as severe local injury. 
069. Pediatric Patient Treated with Outpatient Uridine Triacetate After Capecitabine Exposure

\author{
Marielle Brenner ${ }^{1,2}$, Mary Wermuth ${ }^{1,2}$ \\ ${ }^{1}$ Indiana University School of Medicine, Indianapolis, IN, USA. ${ }^{2}$ Indiana \\ Poison Center, Indianapolis, IN, USA
}

Background: Uridine triacetate was approved by the United States Food and Drug Administration (FDA) in 2015 as an antidote for 5-fluorouracil and capecitabine poisoning. Previous studies on efficacy and safety of uridine triacetate treatment are limited to the hospital setting.

Methods: This is a single-patient chart review. A two-year-old boy was admitted to the hospital after an exposure to capecitabine. He was found with an empty bottle of his grandmother's capecitabine $150 \mathrm{mg}$. He presented asymptomatic with normal vital signs for age. Physical exam was unremarkable. His initial laboratory values included white blood cell count $7.9 \mathrm{k} / \mathrm{cumm}$, hemoglobin $11.9 \mathrm{~g} / \mathrm{dL}$, and platelet count 282 $\mathrm{k} /$ cumm. As capecitabine toxicity has not been studied in children, it was decided that he should receive uridine triacetate per FDA guidelines which required transfer to a tertiary children's hospital that had a limited supply of the antidote. He remained asymptomatic on hospital day two with laboratory values of white blood cell count $12.7 \mathrm{k} / \mathrm{cumm}$, hemoglobin $13.0 \mathrm{~g} / \mathrm{dL}$, and platelet count $328 \mathrm{k} / \mathrm{cumm}$. After discussion with the hospital pharmacy, an outpatient prescription for the remaining doses of uridine triacetate was approved and the patient was discharged with primary pediatrician follow-up.

Results: After discussions with several laboratories, there is no confirmatory testing available for capecitabine exposure, just for genetic predisposition for toxicity. Discharged with a plan for outpatient follow-up and laboratory testing at day nine, 14 , and 21 , the first of which is scheduled for the date of abstract submission. No additional health care visits have been reported to the poison center.

Conclusion: In patients with capecitabine exposures who present asymptomatic with normal complete blood counts, outpatient treatment with uridine triacetate may be an effective treatment method that can decrease overall health care costs.

070. Neurologic Immune Related Adverse Effects (irAE) in Patient Treated with Immune Checkpoint Inhibitors (ICIs)

\author{
Abia Abia ${ }^{1}$, Rubén E Olmedo ${ }^{2}$ \\ ${ }^{1}$ Mount Sinai Hospital, Icahn School of Medicine at Mount Sinai, New \\ York City, NY, USA. ${ }^{2}$ New York City, NY, USA
}

Background: ICIs, such as nivolumab, ipilimumab, and pembrolizumab, are immunotherapies for multiple malignancies. By binding IC proteins (PD-1, CTLA-4, and PD-L1) expressed in cancer cells, these immunomodulators upregulate host antitumor immunity. Frequently occurring irAE of these therapies are pneumonitis, colitis, and rashes. Neuromuscular irAE, such as myasthenia gravis (MG) and myositis, are rare but have notable consequences that can mimic neurological thromboembolic emergencies. Unlike MG or myositis that arise idiopathically, these ICI-mediated sequelae progress quickly and have lower prevalence of sero-markers commonly associated with MG or myositis. Treatments for these irAE include high-dose steroids, IVIg, plasmapheresis, and supportive treatment.

Results: A 56-year-old male with a past medical history of metastatic melanoma on nivolumab and ipilimumab for a month, presented to the ED after complaining of bilateral ptosis, difficulty with handling oral secretions, hoarseness, and weakness of the lower legs progressing over two weeks. The patient denied any infectious symptoms, sensory deficits, dizziness, or ataxia. Physical exam was notable for bilateral ptosis, hoarseness, and slurred speech. His pupils were equal and reactive and his eyelids drooped on upward gaze. Sensation and strength were intact and gait was normal. Laboratory tests revealed mild transaminase elevations and a CPK of $1044 \mathrm{U} / \mathrm{L}$. MRI of the brain and spine showed no evidence of masses, metastatic disease, hemorrhage, stroke, or infarct. Sero-markers for the acetylcholine receptor and muscle specific kinase antibodies were negative. In the ED, the patient's ICIs were stopped and high-dose steroids were started. Within a few days of hospitalization, his neurological symptoms resolved and he was discharged on a prolonged prednisone taper.

Conclusion: MG and myositis are potential irAE of ICI therapy. As these medications are becoming more commonplace, it is important for emergency physicians and toxicologists to recognize neurological complications early in patients on ICIs for appropriate treatment initiation.

\section{Severe Encephalopathy Secondary to Intrathecal Ziconotide} Toxicity

Andrew F Micciche, Rachael C Westover, Carin K Malley, Matthew P Scanlon, Harmony B Yourish, Karen E Olson, Lee J Varelas, Ron M Vidri, John W Evankovich, Joshua A Shulman

University of Pittsburgh Medical Center, Pittsburgh, PA, USA

Background: Ziconotide is a non-opioid analgesic that is a synthetic version of $\omega$-conotoxin M VII A, a neurotoxic peptide found in the venom of Conus cone snail species. Ziconotide has been utilized more frequently for the management of chronic pain.

Hypothesis: Ziconotide toxicity, especially when given intrathecally, may cause protracted, severe encephalopathy.

Methods: This is a single-patient case report. A 35-year-old male with a history of paraplegia and chronic pressure ulcers was admitted for fevers and encephalopathy after months of worsening neuropsychiatric symptoms with initial concern for right hip septic arthritis. His chronic pain had been managed with an intrathecal pump consisting of a combined ziconotide-baclofen solution. Following admission, the patient became increasingly agitated and ultimately required transfer to the ICU with subsequent intubation. Despite an extensive infectious workup and a prolonged empiric course of broad-spectrum antibiotics, no infectious source was identified. The intrathecal pump was interrogated and found to be functioning appropriately. Attempts to wean sedation proved difficult.

Results: After an extensive workup without another clear etiology for his symptoms, ziconotide toxicity was considered. On hospital day 16 , the ziconotide-baclofen solution was removed from the intrathecal pump and replaced with a baclofen-only solution. Encephalopathy improved over the following days, and the patient was extubated on hospital day 19. This clinical improvement is consistent with expected clearance of ziconotide from cerebrospinal fluid. While this case cannot definitively determine causation, resolution of the patient's encephalopathy following removal of ziconotide from the intrathecal pump supports the diagnosis of ziconotide toxicity.

Conclusion: While uncommon, ziconotide toxicity should be considered in patients with intrathecal exposure to ziconotide who develop otherwise unexplained neuropsychiatric symptoms.

\section{Liver Failure from Homeopathic Medication Obtained via Amazon}

Sam T Ontiveros, Bryan Corbett

University of California San Diego, San Diego, California, USA

Background: There is growing interest in ayurvedic, homeopathic, and "natural" medications. Most are readily available and not subject to regulation by the Food and Drug Administration. Many contain unlisted ingredients or concentrations other than advertised.

Hypothesis: Taking commercially available naturopathic and homeopathic remedies risks potentially serious adverse events. 
Methods: A 23-year-old female with a history of polycystic ovary syndrome presented in acute liver failure with grade IV hepatic encephalopathy. The patient had no prescribed medications but had taken homeopathic medications prepared by her Indian physician for two years. Due to COVID-19 restrictions, her supply had run out for 2.5 months. She obtained Ova Tosta $30 \mathrm{C}$ via Amazon 1.5 months prior to presentation. Twenty days later she noticed very orange urine and discontinued use. She developed nausea and malaise and presented to the hospital with an alkaline phosphatase $171 \mathrm{U} / \mathrm{L}$, AST 1,489 U/L, ALT 1,896 U/L, total bilirubin $9.2 \mathrm{mg} / \mathrm{dL}$, albumin $3.6 \mathrm{~g} / \mathrm{dL}$, protein $7.1 \mathrm{~g} / \mathrm{dL}$, and GFR of $>60$ $\mathrm{ml} / \mathrm{min}$. Acetaminophen was undetectable. Ultrasound showed a hypoechoic liver. The patient was seen one week later in gastroenterology clinic and was referred to the hospital in acute liver failure with normal mental status. Two days later she arrived at our center encephalopathic with an AST $213 \mathrm{U} / \mathrm{L}$, ALT $475 \mathrm{U} / \mathrm{L}$, INR of 6.4, ammonia $149 \mathrm{uMol} / \mathrm{L}$, bilirubin $27 \mathrm{mg} / \mathrm{dL}$, creatinine $0.5 \mathrm{mg} / \mathrm{dL}$, and GFR > 60. Acetaminophen was undetectable and iron was normal. Infectious workup was unrevealing and the patient was listed for transplant.

Results: After orthotopic liver transplant the patient has recovered well. She confirms no high risk behaviors, no toxic exposures, and Ova Tosta as her only new medication.

Conclusion: Non-prescription naturopathic and homeopathic medications are increasingly popular and may pose a risk of drug-induced liver injury.

\section{Drug Induced Psychosis Due to Combination of Additive Corticosteroid Use and Selinexor}

\section{Marielle A Brenner ${ }^{1,2}$, Mary E Wermuth ${ }^{1,2}$ \\ ${ }^{1}$ Indiana University School of Medicine, Indianapolis, IN, USA. ${ }^{2}$ Indiana Poison Center, Indianapolis, IN, USA}

Background: Steroid-induced psychosis has been well described from oral corticosteroids and it has been increasingly reported from intramuscular (IM) administration. A newly developed medication for multiple myeloma, an exportin one inhibitor, selinexor, has been recently approved for use in combination with dexamethasone and has been associated with up to a $30 \%$ incidence of altered mental status.

Methods: 48-year-old female with relapsed multiple myeloma was admitted to the hospital with one day of altered mental status. Symptoms included pressured speech, labile emotions, confusion, and insomnia. Vitals were normal and her exam was otherwise unremarkable. She had started a twice weekly regimen of oral selinexordexamethasone $80 \mathrm{mg}-20 \mathrm{mg}$ in the past month. Her two most recent doses coincided with administration of IM triamcinolone acetonide $80 \mathrm{mg}$ for treatment of a pruritic rash at two outpatient visits that occurred two and four days prior to presentation. Laboratory values were unchanged from baseline, including negative acetaminophen and salicylate levels. ECG showed sinus bradycardia and normal intervals. Toxicology and psychiatry were consulted. After evaluation and review of home medications, it was concluded that her presentation was consistent with steroid-induced psychosis. She was started on lorazepam and olanzapine. Her mental status improved on hospital day five and she was discharged on the next day on a short course of olanzapine. Selinexor-dexamethasone therapy was held for four weeks.

Conclusion: This case details steroid-induced psychosis after two recent high-dose intramuscular corticosteroid injections in a patient who recently started a new oral corticosteroid regimen, demonstrating the risk of steroid induced psychosis with additive use of steroids for different indications, which has not been previously reported. While not previously explored to our knowledge, we question whether the high incidence of altered mental status experienced with selinexor is due to its concurrent use with dexamethasone.
074. Inadvertent Intrathecal Digoxin Administration Resulting in Prolonged Neurological Sequelae

Rachael C Westover, Andrew F Micciche, Carin K Malley, Matthew P Scanlon, Joshua A Shulman, Michael G Abesamis

University of Pittsburgh School of Medicine, Pittsburgh, PA, USA

Background: Cardiotoxicity secondary to digoxin is well-documented with predictable effects and available treatment modalities; however, evidence regarding intrathecal digoxin administration is limited to a few case reports.

Hypothesis: Digoxin may cause encephalopathy, seizures, and stroke when administered intrathecally.

Methods: This is a single-patient case study reported to the local poison center. A 36-year-old female underwent routine caesarean section using spinal anesthesia. During the procedure, she received dexmedetomidine and diphenhydramine for sedation in addition to $450 \mathrm{mcg}$ of digoxin intrathecally. Immediately afterward, she endorsed lower extremity weakness. Initial total digoxin level was $0.5 \mathrm{mcg} / \mathrm{L}$. She was given intravenous dexamethasone, ketorolac, and digoxin-specific Fab fragments. Patient was admitted to the ICU and developed progressively worsening upper extremity weakness and respiratory failure requiring intubation. Vital signs remained stable.

Results: The patient was given potassium and magnesium due to mild hypokalemia and QTc prolongation. No dysrhythmia developed. Repeat total digoxin level that night was $1.2 \mathrm{mcg} / \mathrm{L}$; free digoxin level the following day was $<0.3 \mathrm{mcg} / \mathrm{L}$. Patient had a seizure on day two requiring levetiracetam and lorazepam. MRI performed three days after admission revealed a right middle cerebral artery infarct. She was extubated on day six and continued to demonstrate confusion and agitation with speech difficulties. Patient was to be discharged to inpatient rehabilitation but had a seizure and was reintubated; lumbar puncture at that time showed findings concerning for meningitis. Upon ultimate hospital discharge, the patient could speak in full sentences, follow basic commands, and write but continued to have left visual field cuts and neglect in addition to swallowing dysfunction. She did not recognize her child.

Conclusion: Intrathecal digoxin may result in seizure, stroke, and prolonged encephalopathy after inadvertent administration. Treatment is supportive with gradual recovery; digoxin-specific Fab fragments and cerebrospinal fluid lavage are unlikely to be beneficial.

\section{Hypoglycemia After Exposure to GLP-1 Receptor Agonists in Cases Reported to Poison Control Centers}

Faye Girardi ${ }^{1}$, Amber Lin ${ }^{1}$, Robert Hendrickson ${ }^{1,2}$

${ }^{1}$ Oregon Health and Science University, Portland, OR, USA. ${ }^{2}$ Oregon Poison Center, Portland, OR, USA

Background: Glucagon-like peptide-1 receptor agonists (GLP-1RA) are medications primarily used to treat patients with diabetes mellitus. GLP1RA have multiple effects which include increasing insulin secretion. Hypoglycemia has been reported with routine use of these medications. Poison centers consulted with exposures to GLP-1RA have to determine risk of adverse effects in diverse patient populations.

Hypothesis: What factors are associated with hypoglycemia after exposure to GLP-1RA?

Methods: This is a case control study of patients reported to U.S. Poison Centers (NPDS database) between 2005 and 2018 with exposure to GLP1RA. Cases were those with hypoglycemia and controls those without. Categorical data was compared using chi-square or Fisher exact test and continuous data using $t$-test. Inclusion criteria included single-agent exposures with an associated product and FDA approved formulation. Cases were excluded if essential coding was missing and if routes were not parenteral. 
Results: A total of 3171 cases were identified and 2527 were included for analysis. In total, 138 cases of hypoglycemia were identified. $63.5 \%$ (87) involved patients $18-59$ years old and $36.5 \%$ (50) were $\geq 60$ years. A 10 fold dosing error was more common in patients with hypoglycemia than it was in patients without hypoglycemia $(2.9 \%$ versus $0.5 \%, p<0.01)$. Exenatide immediate release was most commonly associated with hypoglycemia $65.9 \%$ (91/138). 64.5\% (89) received food/snack and $23.9 \%$ (33) glucose. A total of 114 cases included data on duration of clinical effect. $24.6 \%$ had duration $\leq 2 \mathrm{~h}, 42.1 \% 2$ to $\leq 8 \mathrm{~h}, 28.9 \% 8$ to $\leq 24 \mathrm{~h}$, and $4.4 \% 24 \mathrm{~h}$ to $\leq 1$ week.

Conclusion: Hypoglycemia occurred in $5.5 \%$ of cases; however, all cases of hypoglycemia occurred in adult patients who may be on other hypoglycemic medications. The proportion of patients who became hypoglycemic was approximately equal between age groups $18-59(5.7 \%)$ and $60+(5.6 \%)$. None of the exposures in pediatric patients (76) resulted in hypoglycemia.

076. Gabapentin Adverse Events: a Descriptive Analysis of the Food and Drug Administration Adverse Events Reporting System (FAERS)

Paiboon Jungsuwadee

Fairleigh Dickinson University School of Pharmacy \& Health Sciences, Florham Park, NJ, USA

Background: Prescriptions of gabapentin have increased exponentially over the years. According to The Food and Drug Administration Adverse Event Reporting System (FAERS) database, reports of gabapentinassociated adverse events continue to increase overtime since its initial approval in 1993. This study describes the nature and extent of gabapentin adverse events as well as potential risk factors utilizing the FAERS database.

Methods: List of adverse event cases associated with gabapentin was downloaded from the FAERS database (data as of September 30, 2020). Raw data were systematically manipulated and organized to exclude missing or "Not specified" reported values. The data set were subjected to analyses using the RStudio package.

Results: The total entries subjected to analyses were 9665 cases. These reports covered gabapentin adverse events occurring in 48 countries, which increased overtime. Seventy-two percent were adverse events that occurred in the US. Two-third of cases were female (64.9\%), and twothird were reported as "serious" (66.6\%). The reported patient outcomes were classified into seven categories, namely, "congenital anomaly", "died", "disabled", "life threatening", "non-serious", "other serious outcomes" and "required intervention". The percentages of cases for each category were $0.1 \%, 4.5 \%, 4.9 \%, 3.8 \%, 33.4 \%, 33.5 \%$ and $19.8 \%$, respectively. The analyses showed that adverse events occurred most frequently in patients' weight 51-100 kilograms, and age 41-60 years. Of this same age bracket, $26.9 \%$ female patients versus $14.8 \%$ male patients died as the outcome associated with the use of gabapentin.

Conclusion: As the use of gabapentin continues to grow each year, so does its adverse events. Of 9665 cases analyzed, 66.6\% of adverse event outcomes are serious, which may be affected by certain factors such as patients age, weight and sex. The report in the time frame analyzed prompts the need for further investigation to examine risk factors that are better predicting a seriousness of gabapentin adverse events.

\section{Characteristics of Unintentional Gabapentinoid Exposures}

Elizabeth M Silver ${ }^{1}$, Michael Darracq ${ }^{2}$, Stephen L Thornton ${ }^{1}$ ${ }^{1}$ Kansas Poison Control Center at The University of Kansas Health System, Kansas City, KS, USA. ${ }^{2}$ University of California San Francisco-Fresno Department of Emergency Medicine, Fresno, California, USA
Background: Gabapentin and pregabalin are antiepileptics with structural similarity to gamma-aminobutyric acid used for a variety of conditions. Despite a steady increase in prescriptions, there remains a paucity of information regarding pediatric exposures, with no comparative studies between them. This study aims to report the characteristics of unintentional pediatric poisonings involving gabapentin and pregabalin to the National Poison Data System (NPDS) over 10 years.

Methods: The NPDS was queried for all single substance unintentional pediatric (ages 19 and under) exposures to gabapentin or pregabalin from January 1,2009 , to December 31, 2019. All data was analyzed using IBM Statistics for Mac (v. 25, IBM Corporation, Armonik, NY).

Results: A total of 12485 cases were identified. Ninety-seven percent $(n$ $=12066)$ of cases involved gabapentin. Fifty-two percent $(n=6546)$ of all cases were followed to a known outcome. Gabapentin cases followed to a known outcome increased steadily from 449 in 2009 to 710 in 2019 , while pregabalin did not (20 exposures in 2009 and 14 in 2019). Ingestion was the most common route. Ages 1-2 years made up the largest age group exposed to gabapentin $(n=4128)$ and pregabalin $(n=$ 148). For all ingestions, "Not followed-minimal clinical effects possible", "Not followed-judged as non-toxic exposure", and "No effect" made up $86 \%$ of gabapentin outcomes compared to $80 \%$ of pregabalin outcomes. The most common symptom experienced for both exposures was drowsiness/lethargy. Most ingestions were managed onsite for gabapentin and pregabalin ( $70 \%$ vs. $57 \%$, respectfully). Patients were referred into a healthcare facility in $12 \%$ of pregabalin ingestions compared to $9.4 \%$ of gabapentin ingestions. A higher percent of pregabalin cases were admitted to critical care units (1.5\% vs. $1.6 \%)$. No deaths were reported.

Conclusion: Gabapentin exposures are more commonly encountered compared to pregabalin. Both exposures were similar regarding demographics, clinical effects, outcomes and level of care needed for management.

\section{Acute Pulmonary Embolism Associated with Chronic Nitrous Oxide Use}

Samuel J Wagner, Zhanna Livshits

University of California San Francisco, San Francisco, CA, USA

Background: Nitrous oxide is commonly misused for its euphoric effects and is readily accessible in the form of "whippets." In chronic use, nitrous oxide induces megaloblastic anemia and posterior column sensorimotor polyneuropathy via inactivation of vitamin B12, a necessary cofactor for methionine synthase, leading to accumulation of substrates homocysteine and methylmalonic acid. Hyperhomocysteinemia, either genetic or acquired, is associated with venous thromboembolism.

Methods: This is a single-patient case report. A 66-year-old male presented to the emergency department for syncope and was found to have a submassive pulmonary embolism. He endorsed using hundreds of whippet canisters per day for many years. He exhibited neuropsychiatric symptoms of weakness, ataxia, paresthesias, agitation, and memory loss; his bedside exam was consistent with subacute combined degeneration of the spinal cord. He was mildly anemic with a hemoglobin of $13.2 \mathrm{~g} / \mathrm{dL}$ (MCV of $98 \mathrm{fL}$ ). The patient's homocysteine concentration was 67 $\mu \mathrm{mol} / \mathrm{L}(4-14 \mu \mathrm{mol} / \mathrm{L})$ and methylmalonic acid concentration was 1.25 $\mu \mathrm{mol} / \mathrm{L}(0.00-0.40 \mu \mathrm{mol} / \mathrm{L})$, while vitamin B12 was $1540 \mathrm{ng} / \mathrm{L}(301-$ $816 \mathrm{ng} / \mathrm{L})$ and folate $18.4(>4 \mathrm{ng} / \mathrm{mL})$. He had negative testing for common causes of hypercoagulability including undetectable antibodies to beta-2-glycoprotein, anti-cardiolipin antibody, and lupus anticoagulant, as well as COVID-19. Antithrombin III activity was normal as well as concentrations of free protein $\mathrm{C}$ and $\mathrm{S}$. The patient was discharged on rivaroxaban.

Results: Chronic nitrous oxide use may predispose to a hypercoagulable state with a plausible mechanism being the inactivation of B12 leading to acquired hyperhomocysteinemia. To our knowledge, this is one of two 
reported cases of chronic nitrous oxide use associated with pulmonary embolism where other causes of hypercoagulability were excluded including COVID-19.

Conclusion: We report a case of pulmonary embolism associated with chronic nitrous oxide use.

079. Nitrous Oxide Abuse Induced Subacute Combined Spinal Cord Degeneration Despite Patient Initiated Oral B12 Supplementation in Three Patients

Courtney Temple, John Thompson, Matthew Correia, Annette Lopez, Robert Hendrickson, B Zane Horowitz

Oregon Health \& Science University, Portland, OR, USA

Background: Nitrous oxide, a compressed gas, is a commonly used inhalation anesthetic. It is legal to purchase as a food additive and is popular as a recreational drug given its euphoric effects. Abuse can cause functional B12 deficiency, leading to clinical features and MRI findings consistent with subacute combined spinal cord degeneration (SCD). We present three patients with progressive neurotoxicity in the setting of nitrous oxide cartridge or "whippet" abuse and self-reported B12 supplementation.

Methods: The Oregon Poison Center (OPC) medical records and imaging from these patients were reviewed. Patient 1: A 37-year-old male with years of whippet abuse presented with severe extremity paresthesias over two weeks of increased use. Patient 2: A 33-year-old male with daily whippet abuse (up to 50 cartridges) presented with several days of progressive lower extremity sensory changes. Patient 3: A 31-year-old female with a three month history of heavy whippet abuse presented with several days of severe lower extremity numbness, and inability to ambulate.

Results: All patients reported increased use of whippets during COVID quarantine and began supplementing Vitamin B12 at the recommendation of online forums after development of symptoms. All patients were found to have elevated B12 though exhibited elevated concentrations of both homocysteine and methylmalonic acid. Patients 1 and 3 had MRI findings of T2 hyperintensity in the cervical and thoracic dorsal columns consistent with SCD. MRI in patient 2 was limited by motion artifact. No patients demonstrated a folate deficiency or significant macrocytic anemia; however, two had an elevated RDW.

Conclusion: Nitrous oxide abuse has been documented to cause functional B12 deficiency and subsequent subacute combined spinal cord degeneration. While oral B12 supplementation may prevent or treat anemia, it does not seem to mitigate neurotoxicity based on this case series.

\section{Pediatric Cannabis Product Ingestions: Ranges of Exposure,} Effects, and Outcomes

Eric E Kaczor, Bonnie L Mathews, Kara L LaBarge, Brittany P Chapman, Stephanie P Carreiro

University of Massachusetts Medical School, Worcester, Massachusetts, USA

Background: Pediatric edible cannabis exposures have been increasing in recent years, and are associated with respiratory depression and the need for hospital admission. The incidence of these outcomes and their relationship to exposure/patient characteristics have not been fully described.

Research Question: In pediatric patients exposed to edible cannabis, how do patient and ingestion characteristics affect the need for respiratory support and hospital admission?

Methods: This is a retrospective chart review conducted at a single, tertiary care academic medical center over a 28 -month period. Inclusion criteria were: evaluation in the ED, age less than 18 years, and physician documented exposure to edible cannabis. Patients with known or suspected co-ingestion of other substances were excluded. Cases were screened and independently evaluated by two investigators using a standardized data abstraction tool. Descriptive statistics were calculated for predictor and outcome variables using Student's $t$-test and Wilcoxon Ranks Sum for continuous variables and the chi-square test for categorical variables.

Results: Thirty-two cases meeting criteria were identified, 53\% female with a median age of 12.3 years. The incidence of need for respiratory support was $16 \%(N=5)$ and the incidence of hospital admission was $34 \%(N=11)$. Age less than 10 years was associated with bradypnea, hypertension, hospital admission, and need for respiratory support. The need for respiratory support was also significantly associated with female sex, seizure, lethargy/somnolence, and hypertension. Overall admission was associated with lower weight, younger age, lethargy/somnolence, unintentional ingestions, and hypertension. There was a five-fold increase in the number of pediatric edible cannabis exposures after recreational cannabis dispensaries opened in Massachusetts.

Conclusion: Younger age was associated with respiratory support and hospital admission. Overall, there was a low incidence of seizures and need for respiratory support, but hospital admission was common. Pediatric edible cannabis exposures occurred with increasing frequency over the study timeframe.

\section{Comparative Analysis of Cannabis Toxicity by Route of Patient Exposure}

Brian Lewis, Tiffany Fleeger, Bryan Judge, Brad Riley, Jeffrey S Jones Spectrum Health - Michigan State University Emergency Medicine Residency Program, Grand Rapids, MI, USA

Background: The state law to legalize recreational use of marijuana in Michigan went into effect in December 2018.

Hypothesis: The clinical effects and toxicity associated with marijuana edibles differ from inhaled marijuana in our emergency department (ED) population during this early legalization period.

Methods: This was a retrospective comparative analysis of consecutive patients diagnosed with toxicity related to inhaled versus edible cannabis (food products, beverages). Patients were seen at seven EDs over a 20month study period. Affiliated institutions included three rural medical centers, three university hospitals and a children's tertiary care facility. Data collected included demographics, pre-hospital care, product ingested, dose form, coingestants, and clinical findings. Chi-square and $t$-tests were used to compare the two study groups across key demographic and clinical variables.

Results: During the study period, 909 patients were evaluated for THC toxicity; 155 visits $(17.1 \%)$ were attributable to edible cannabis. Although patients with visits attributed to edible cannabis were older (30.5 versus 27.9 years, $p=0.022$ ), the age range was broader. Sixteen $(10.3 \%)$ were $>64$ years and nine $(5.8 \%)$ were children $<12$ years of age. The most common ingestions were brownies and cookies, followed by candy and beverages. Visits attributable to inhaled cannabis were more likely to be for cannabinoid hyperemesis syndrome $(33.8 \%)$ or intoxication $(28.8 \%)$. In contrast, visits due to edible cannabis had a wide range of psychiatric $(27.1 \%)$, cardiovascular $(21.9 \%)$, and neurologic $(14.8 \%)$ complaints. Patients with visits attributable to inhaled cannabis had fewer comorbidities but were more likely to have a history of polysubstance use $(22.4 \%$ versus $7.7 \%, p<0.0001$ ).

Conclusion: ED visits attributable to cannabis toxicity have increased following legalization of marijuana in our community. Edible cannabis accounted for over $17 \%$ of these visits and had a different demographic profile and presentation compared to inhaled cannabis. 
082. Marijuana Exposure Trends in Canadian Children in the Pre, Peri and Post- Legalization Era

Neta Cohen ${ }^{1,2}$, Laura Galvis Blanco ${ }^{1,2}$, Adrienne Davis ${ }^{1,2}$, Alyssa $\mathrm{Kahane}^{3}$, Mathew Mathew ${ }^{1,2}$, Inbal Kestenbom ${ }^{1,2}$, Gidon Test ${ }^{1,2}$, Yehonatan Pasternak $^{1,2}$, Maggie Rumantir ${ }^{1,2}$, Yaron Finkelstein ${ }^{1,2}$

${ }^{1}$ The Hospital for Sick Children, Toronto, Ontario, Canada. ${ }^{2}$ University of Toronto, Toronto, Ontario, Canada. ${ }^{3}$ University of Ottawa, Ottawa, Ontario, Canada

Background: In November 2017, the legislation of recreational cannabis use was passed by the Canadian House of Commons (peri legalization period). On October 17, 2018, Canada became the second country in the world to formally legalize recreational use of cannabis.

Hypothesis: The number of marijuana exposure cases and the clinical severity (i.e., intensive care unit [ICU] admissions) among Canadian children will be increased in the peri-post legalization period, compared to the pre legalization period.

Methods: A retrospective, single center cohort study, investigating patients aged $0-18$ years with accidental or intentional marijuana exposure, who presented to the emergency department of The Hospital for Sick Children, Toronto, from January 1, 2008, to December 31, 2019, and whose presenting symptoms were attributed to marijuana use. Two-time frames were defined: Pre legalization period - from January 2008 to October 2017 and Peri-post legalization period - from November 2017 to December 2019. We collected data regarding the exposure circumstances, clinical manifestations, management, disposition, and outcomes. Results: Two hundred ninety-eight patients were included. The number of children presenting with marijuana exposure did not differ between the pre vs. peri-post legalization period (2.04/month vs. $2.07 /$ month; $p=\mathrm{NS}$ ). More patients were admitted to the ICU in the peri-post legalization period, compared to the pre-legalization period $(15.0 \%$ [8/53] vs. $4.9 \%$ [12/241], respectively; $p=0.02$ ). Ingestion as mode of exposure was more common in the peri-post period, compared to the pre legalization period $(18.8 \%$ [10/53] vs. $6.6 \%$ [16/241], $p=0.01)$.

Conclusion: Marijuana exposure severity in children presenting for emergency care has significantly increased in association with its nationwide recreational use legalization.

083. Unintentional Pediatric Tetrahydrocannabinol Ingestions in Massachusetts Following Establishment of Recreational-Use Dispensaries: a Poison Center Study

Alexander F Barbuto ${ }^{1,2}$, Michele M Burns ${ }^{1,3}$

${ }^{1}$ Harvard Medical Toxicology Program, Boston, MA, USA. ${ }^{2}$ Carl R. Darnall Army Medical Center, Ft. Hood, TX, USA. ${ }^{3}$ Boston Children's Hospital, Boston, MA, USA

Background: Several states have reported increased rates of pediatric unintentional exposures to marijuana following legalization of recreational use. Edible tetrahydrocannabinol (THC) products may pose unique risks. Voters legalized recreational marijuana in the Commonwealth of Massachusetts in November 2016, and the Cannabis Control Commission drafted regulations prior to the first dispensaries for adultuse marijuana opening in November 2018; the effects on unintentional ingestions from legislation in the Commonwealth have not been described.

Research Question: How has the incidence of pediatric unintentional edible THC exposures changed after the first dispensaries opened in Massachusetts?

Methods: This is a retrospective review of Poison Control Center cases. We reviewed records from the Regional Center for Poison Control and Prevention Serving Massachusetts and Rhode Island (PCC) from the 18month periods before and after the first adult-use dispensaries opened
(November 20,2018). We included poison center cases involving children less than 13 years old with edible THC exposure.

Results: From November 20, 2018, through May 20, 2020, our PCC received 79 reported cases within Massachusetts for children under 13 years old who ingested edible-THC products, compared with 46 cases in the 18-month period prior to November 20, 2018 (71\% increase). Over the same periods, the total number of calls to the PCC for this age group rose from 27,286 to 31,997 (17\% increase). These do not include ingestion of THC vaping liquids or plant material. Of these most recent 79 cases, 11 were admitted to an intensive care setting and an additional 20 were admitted to a pediatric ward. The remaining were evaluated and observed in an emergency department. Eighteen children required transfer from community hospitals to pediatric hospitals.

Conclusion: Despite thoughtful regulation by the Massachusetts Cannabis Control Commission, unintentional pediatric exposures to edible THC products rose after the first dispensaries opened in the Commonwealth.

084. Dynamics of Cannabis Exposure after Legalization of Marijuana for Recreational Usage

Michael Chary ${ }^{1,2}$, Daniel Overbeek ${ }^{3}$, Michele M Burns ${ }^{2}$

${ }^{1}$ Weill Cornell, New York, New York, USA. ${ }^{2}$ Boston Children's Hospital, Boston, MA, USA. ${ }^{3}$ Boston Children's Hospital, Boston, MA, USA

Background: There is a concern that an increase in the availability of cannabis-containing products will lead to increased exposure and toxicity. Studies of calls to Poison Control Centers in Massachusetts and Colorado noted an increase in pediatric exposures to products containing cannabis after the legalization of marijuana for medical purposes.

Methods: Surveys of $8^{\text {th }}-12^{\text {th }}$ graders found minimal to no change in adolescent marijuana use after the legalization of marijuana for recreational use. We obtained 355, 957 unique calls to MARI PCC from August 29,2012 , to October 12, 2019. We excluded 35,008 calls in which no substance was recorded and an additional 9,757 calls in which no age was recorded. We identified 1,426 calls that involved a cannabis-containing product, of which 853 involved only one cannabis-containing substance. The remaining 573 calls involved at least two substances, at least one of which contained cannabis.

Results: The annualized rate of calls about cannabis-related products increased from 120 calls per year before the legalization of marijuana for recreational use to 352 calls per year after the first dispensary opened. All age groups noted an increase in exposures, although the magnitude of the increase was statistically significant for only those $0-6$ and 13-19 years of age, even after correcting for simultaneous multiple comparisons $(p<0.001$, - test, Bonferroni correction factor 6).

Conclusion: The major finding of this study is that reported ingestions of cannabis-containing products increased after stores selling marijuana for recreational purposes opened. The increase was driven by unintentional exposures in children between 0 and 19 and a less substantial but still significant increase in those older than 50 .

\section{Kratom Withdrawal Treated with Buprenorphine}

Daniel R Lasoff, Garret Winkler

University of California, San Diego, San Diego, CA, USA

Background: Kratom is a drug with increasing popularity for recreational use and self-treatment for opioid use disorder and opioid dependence. Kratom withdrawal is a potential complication of discontinuation after long-term use of Kratom.

Hypothesis: Buprenorphine is a possible treatment modality for Kratom withdrawal. 
Methods: This is a case report of two patients treated with buprenorphine for Kratom withdrawal. Case 1: a 37-year-old female who has been using Kratom for two years for restless legs presents to the ED noting dysphoria, visual hallucinations, and suicidal ideation. She notes over the past six months, her Kratom has not been working as well, so she has increased daily use to several times per day. She presents with signs of opioid withdrawal as well as vivid visual hallucinations. Her initial COWS score is 14. A urine drug screen was negative, and her urine was positive for mitragynine. Case 2: a 35-year-old female with a history of opioid use disorder 10 years previously, using Kratom for one year to stop opioids, presents after discontinuing Kratom four days ago. She completed a five day chlordiazepoxide taper and presented to the ED with withdrawal symptoms that included abdominal pain, myalgias, vomiting, and diarrhea. On arrival her COWS score was 16.

Results: Case \#1 was treated with $16 \mathrm{mg} / 2 \mathrm{mg}$ of buprenorphine/ naloxone with improvement of her COWS score to three. Her hallucinations and dysphoria also resolved. Case \#2 was treated with two separate doses of $4 \mathrm{mg} / 0.5 \mathrm{mg}$ buprenorphine/naloxone with improvement of her COWS score to two. Both patients were discharge home and doing well in follow up by phone call two days later.

Conclusion: Buprenorphine should be considered as a treatment for Kratom withdrawal and use disorder.

086. Re-Routing: Buprenorphine Microinduction Using Intravenous Formulation Sublingually

Andrew M King ${ }^{1,2}$, Asim Kagzi ${ }^{1}$, Mohammed Elbashir ${ }^{1}$, Bram A Dolcourt $^{1,2}$, Varun Vohra ${ }^{2}$, Elizabeth Jacobs ${ }^{3,2}$, Krista Wahby ${ }^{3,1}$

${ }^{1}$ Wayne State University, Detroit, MI, USA. ${ }^{2}$ Michigan Poison Center, Detroit, MI, USA. ${ }^{3}$ Detroit Medical Center, Detroit, MI, USA

Background: Algorithms exist to transition patients with opioid use disorder (OUD) to buprenorphine, termed "microinduction". We report a case of microinduction, using intravenous (IV) formulation buprenorphine administered sublingually (SL), from methadone that was well-tolerated without withdrawal.

Hypothesis: IV formulation of buprenorphine, administered SL, can be used for successful microinduction for patients on methadone.

Methods: We report a case of a 45-year-old man with severe OUD and IV drug use who was transitioned from hospital-initiated methadone to buprenorphine over eight days per patient preference. Patient was initially admitted to the hospital with MRSA pneumonia warranting mechanical ventilation and requiring a tube thoracostomy for parapneumonic effusion drainage. Methadone was started in the ICU 13 days prior to microinduction (highest dose $60 \mathrm{mg}$ four days prior to induction) due to difficulty weaning sedation. After extubation, the patient requested transition to buprenorphine. Methadone weaned to $30 \mathrm{mg}$ daily over four days in anticipation of microinduction. Once he reached $30 \mathrm{mg}$ methadone daily, $0.21 \mathrm{mg}$ IV buprenorphine $(0.3 \mathrm{mg} / \mathrm{mL})$ was administered SL via an oral syringe. Dose was doubled each day over four days to $1.6 \mathrm{mg}$ total daily dose. The patient transitioned to SL buprenorphine tablets on day five $(2 \mathrm{mg})$ and dose doubled until reaching a divided daily dose of 16 $\mathrm{mg} / \mathrm{d}$. We continued methadone wean at $5 \mathrm{mg}$ daily to $15 \mathrm{mg}$, which continued for three days during buprenorphine induction, and stopped day eight of buprenorphine induction. The patient used $10 \mathrm{mg}$ oxycodone once daily starting day two through eight for pain at the chest tube site. He denied withdrawal symptoms throughout his course.

Conclusion: IV buprenorphine can be administered SL for microinduction of buprenorphine from methadone and could be considered an alternative to or when buprenorphine patches are unavailable.

087. Successful Ambulatory Buprenorphine Microinduction in an Outpatient, "Terrified of Precipitated Withdrawal" During COVID-19
Timothy J. Wiegand ${ }^{1}$, Mayra Rodriguez ${ }^{2}$

${ }^{I}$ Department of Emergency Medicine, University of Rochester School of Medicine \& Dentistry, Rochester, NY, USA. ${ }^{2}$ Huther Doyle, Rochester, NY, USA

Background: Fentanyl adulteration has increased the risk of precipitated withdrawal. Theories as to why this occurs suggest fentanyl-altered pharmacology with high dose repetitive use. We describe a BUP microinduction, starting with sub-milligram doses, for an outpatient unable to stop fentanyl, using a mixture of telehealth and in-person support during COVID-19.

Hypothesis: Buprenorphine microinduction allows for initiation even if patients are unable to stop use of fentanyl.

Methods: Single-patient chart review. A 24-year-old M with "20 bundles of fentanyl/day" left detoxification against medical advice 3 times due to "terror of precipitated withdrawal." After this, a SL microdosing regimen starting with $1 / 16$ th $(125 \mathrm{mcg})$ of a $2 / 0.5 \mathrm{mg}$ BUP/Naloxone film/ $/ 6$ hours was initiated with doses doubling/day $\rightarrow 250 \mathrm{mcg} / 6$ hours $\rightarrow 0.5 \mathrm{mg} / 6$ hour $\rightarrow 1 / 0.25 \mathrm{mg} / 6$ hours $\rightarrow 2 / 0.5 \mathrm{mg} / 6$ hours $\rightarrow 8 / 2 \mathrm{mg}$ BID. The patient was seen every-other-day on site and via telehealth alternate days. After day one, he reported 5-10 bags of fentanyl/day and on day two no withdrawal symptoms using 3-4 bags "mainly because of habit." At 8/2 mg SL BID, he missed his appointment, arrested for "outstanding warrants." Regardless, he was eager to "tell others" about microinduction stating, "this was amazing I could restart my 'Subs' and not be thrown into withdrawal!"

Results: Microdosing involves initiation of sub-milligram BUP doses while full agonists are continued. Initially called the "Bernese Method," subsequent reports detail varied protocols as patients are continued on high doses of full agonists. Microdosing in the outpatient setting is less controlled. Patient-centered care and establishment of rapport is critical for outpatient microinductions to be successful.

Conclusion: We show that a heavily dependent fentanyl user, terrified of precipitated withdrawal, can start buprenorphine safely. BUP microinduction can revolutionize the induction process allowing for initiation immediately upon patient assessment. Microinduction has important utility during COVID-19.

088. 'Start Low and Delay Until in Withdrawal' - Lessons Learned from Precipitated Withdrawal after Early Buprenorphine Initiation after Methadone

Elaine P Rigney ${ }^{1}$, Timothy J Wiegand ${ }^{2}$

${ }^{1}$ University of Rochester - Strong Memorial Hospital, Rochester, NY, USA. ${ }^{2}$ University of Rochester Medical Center, Rochester, NY, USA

Background: Buprenorphine is a potent partial mu-opioid receptor agonist. While generally considered safe, toxicity can occur in patients taking long-acting or sustained-release (SR) opioids. We describe a case of severe precipitated withdrawal from high-dose buprenorphine without adequate wash-out from methadone and SR-oxymorphone and treatment for precipitated opioid withdrawal.

Hypothesis: High-dose buprenorphine initiated close to full agonists, like methadone, will cause precipitated withdrawal.

Methods: Single-patient chart review. A 55-year-old male with debilitating chronic post-surgical pain and radiation-induced neuropathy from prostate cancer treatment. He gradually escalated to $70 \mathrm{mg} /$ day methadone and $200 \mathrm{mg}$ /day oxymorphone without significant relief; his pain provider considered buprenorphine. Patient was instructed to stop methadone 18 hours (30 mg noon dose, $20 \mathrm{mg} \mathrm{AM}$ ) and SR-oxymorphone $40 \mathrm{mg}$ morning prior to induction. Patient was supervised taking eight $\mathrm{mg}$ buprenorphine and, "15 minutes later [his] ears started to get warm and [doctor] told me to take another $4 \mathrm{mg}$." Subsequently, diffuse pain, cramping, muscle spasms, and myoclonic jerks occurred. His wife 
reported, "he was flailing and screaming for help!" Midazolam and another four mg buprenorphine were administered.

Results: Patient arrived at ED confused, diaphoretic, dyspneic, and tremulous. He received clonidine and lorazepam in $0.2 \mathrm{mg}$ and two $\mathrm{mg}$ doses incrementally with ketorolac and dextrose-containing fluids. He required ICU admission. Symptoms stabilized with repeated doses of clonidine, lorazepam, and adjunctive agents. On day four, buprenorphine was reinitiated with slow titration to $8 / 2 \mathrm{mg}$ SL-TID by discharge.

Conclusion: It is critical to allow sufficient time before initiating buprenorphine after cessation of full agonists. If no washout, try lower dose buprenorphine (e.g., 'micro-induction'). When precipitated withdrawal occurs, we recommend using sympatholytics, sedatives, with reinitiation of low-dose buprenorphine. High doses of buprenorphine given shortly after full agonists will precipitate severe withdrawal. Complicated buprenorphine initiations should involve guidance from experienced providers.

\section{Fentanyl Trumps Methadone}

\section{Jason Hack}

East Carolina University, Greenville, NC, USA

Background: Methadone is a synthetic, long-acting, mu-receptor agonist used as opioid replacement therapy for heroin for $>50$ years. Methadone's attributes to decrease heroin use include limiting craving, withdrawal, and interfering with sought-after positive reinforcement ('high') when uptitrated through treatment.

Methods: This was a non-sequential, observational case series presenting to an urban ED for opioid overdose requiring emergency medical services administration of naloxone over two weeks. Three male and one female patients between 28 and $52 \mathrm{y} / \mathrm{o}$ who reported prior intravenous (IV) opioid use and were currently on methadone $(100-180 \mathrm{mg} /$ day $)>$ eight months were included. Medical work ups were normal; drug screens were positive only for methadone and fentanyl. Each reported they had lapsed and intentionally self-administered fentanyl IV for euphoria and stated a version of: "Everyone knows fentanyl cuts through methadone...so I can still get high."

Results: Methadone's historically salutary effects in patients using illicit opioids may be limited in the current milieu of newly available high potency opioids. Fentanyl is a high potency $(100 \times$ morphine $)$ synthetic anesthetic approved by FDA for use in 1972 and is currently the most common drug associated with illicit overdose deaths in the US. Recent studies have reported positive fentanyl testing in up to $80 \%$ of patients entering opioid replacement therapy, and is also found on surveillance testing during methadone therapy. No clear explanation for the latter results has been proposed or tested in the literature. This case series provides one explanation. Limitations: Once center, limited participants in a short duration case series.

Conclusion: Current opioid replacement therapy with methadone in patients that use, or are directed to use fentanyl by their cohort, may still achieve euphoric reinforcement and therefore are at risk for illicit opioid use and death. This small sample may represent a much larger unrecognized immediate health risk.

\section{Changes in Controlled Substance Prescriber Behavior After Implementation of Michigan's Controlled Substance Laws}

Bram A Dolcourt ${ }^{1}$, John Tarchick ${ }^{1}$, Andrew King ${ }^{1}$, Kyle C Mangan ${ }^{2}$, Shooshan Danagoulian ${ }^{1}$

${ }^{1}$ Wayne State University, Detroit, Michigan, USA. ${ }^{2}$ Detroit Medical Center, Detroit, Michigan, USA

Background: Michigan enacted new controlled substance legislation on June 1, 2018. Legislated changes included requiring providers to review the prescription monitoring program prior to prescribing a controlled substance and certify counselling on opioid use was performed prior to prescribing an opioid. The effect of these changes on prescriber behavior is unknown.

Hypothesis: We expected the new requirements to dramatically reduce controlled substance prescriptions in all specialties.

Methods: This is a retrospective study. 99,606 adult in-patient and ED discharge prescriptions, 82,231 for opioid and 17,375 for benzodiazepines, from August 1, 2016, and March 31, 2019, were extracted from the EMR of a five-facility urban hospital system. Data was converted to morphine or lorazepam equivalents for comparison. Changes in prescriber behavior before and after legislation were evaluated using regression discontinuity design analysis.

Results: Prescribing behaviors of specialties were analyzed: Emergency (EM), Family, and Internal Medicine, General Surgery, Orthopedics, and Obstetrics/Gynecology. Specialties did not respond to legislation uniformly. EM significantly reduced prescribed morphine-equivalents prelegislation, followed by a dramatic drop post-legislation. Other specialties showed limited overall reduction in morphine-equivalents prescribed and wrote fewer "outlier" prescriptions. Family Medicine increased morphine-equivalent prescriptions post-legislation. There is evidence of substitution from opioids to benzodiazepines as shown by increased rates of lorazepam-equivalent prescriptions after the legislation, mainly in EM. Conclusion: The introduction of new regulatory requirements for the prescription of controlled substances showed a significant change in prescriber behavior in most specialties in the hospital system. There was an unexpected increase in lorazepam equivalents in some specialties. It is not known if the change in prescription behavior is motivated by paperwork hurdles and regulatory hassle, or by change in attitude towards opioid prescriptions and increased recognition of opioid use disorder. It is unclear what effect these regulations have on outpatient providers.

\section{Buprenorphine Prescription Challenges During the COVID-19 Pandemic}

Joanne C Routsolias ${ }^{1,2}$, Neeraj Chhabra ${ }^{1,2}$, Allen Hollingsworth ${ }^{1}$, Mark B Mycyk $^{1,2}$

${ }^{1}$ Cook County Health, Chicago, IL, USA. ${ }^{2}$ Toxikon Consortium, Chicago, IL, USA

Background: The COVID-19 pandemic has challenged patients with substance use disorder with unstable housing, job insecurity, shelter in place orders, and closure of clinics. We sought to determine how the shelter-in-place order affected buprenorphine-naloxone prescription dispensing in our urban health care system.

Methods: Onsite outpatient pharmacy dispensing records in a large urban county hospital for a period of three months pre- and three months post- a "shelter-in-place" state order were reviewed. Data collected included basic demographics, source of the prescription, and quantity dispensed. Descriptive, $t$-test, and Wilcoxon analyses were used where appropriate. Results: The Illinois state "shelter-in-place" order took effect on 3/21/20. Between $1 / 1 / 20$ and $3 / 20 / 20$, a total of 246 prescriptions for buprenorphine-naloxone were dispensed from the outpatient pharmacy. The average quantity of tablets was 25 [range 1-84], and $3.6 \%$ of all prescriptions were initiated in the emergency department. Between $3 / 21 / 20$ and $6 / 20 / 20$, a total of 192 prescriptions were dispensed (decrease of 28\%), average quantity dispensed was 40 (increase of 60\%) [range 6$90]$, and there was a $22 \%$ increase in prescriptions originating from the emergency department. The difference in mean quantity prescribed in the two periods was significant $(p<.00001)$.

Conclusion: The overall number of prescriptions for buprenorphinenaloxone decreased after the start of the COVID-19 pandemic, but the average number of pills dispensed per prescription significantly increased. More prescriptions originated from the ED and fewer came from the health system's outpatient substance use clinics. These findings 
highlight challenges faced by this cohort of patients and may be associated with the increased number of overdose deaths reported during this same period. How telehealth availability, increased ED traffic, and other health access strategies can enhance substance use disorder treatment during a pandemic warrants further study.

092. Acute Opioid Overdose Following Nasal Atomization of a Novel Designer Opioid, 2-Methyl AP-237, With Potentiation of Designer Benzodiazepines, Pyrazolam and Clonazolam

Michael $\mathrm{Yeh}^{1,2}$, Alex Krotulski ${ }^{3}$, Colin Therriault ${ }^{1,2}$, Matthew D Eisenstat $^{1,2}$, Harrison Nguyen ${ }^{4}$, Donna Papsun ${ }^{5}$, Melissa H Gittinger ${ }^{1,2}$ ${ }^{1}$ Emory University, Atlanta, GA, USA. ${ }^{2}$ Georgia Poison Center, Atlanta, GA, USA. ${ }^{3}$ Center for Forensic Science Research \& Education, Willow Grove, PA, USA. ${ }^{4}$ Saint Louis University, St. Louis, MO, USA. ${ }^{5}$ NMS Labs, Horsham, PA, USA

Background: 2-Methyl AP-237 is an analogue of bucinnazine (AP-237), an acyl piperazine opioid first synthesized in Japan in 1968. Bucinnazine is used as an analgesic for cancer-related pain in China but not approved for use in the United States. 2-Methyl AP-237 was first identified in illicit drugs seized in Slovenia in 2019. It is sold online as a "research chemical," with discussions among users on Internet drug forums. We present a case of opioid toxidrome associated with use of 2-methyl AP-237 in the setting of polydrug use.

Methods: A 31-year-old male was found unresponsive at home with respiratory depression. He used a nasal spray containing one gram of 2methyl AP-237 dissolved in $30 \mathrm{~mL}$ of water four hours earlier. EMS administered naloxone $0.4 \mathrm{mg} \mathrm{IV}$, then $0.8 \mathrm{mg}$, with improvement of his respiratory and mental status. In the ED, he was somnolent but arousable and oriented. He reported taking methadone earlier that day, and was a chronic user of benzodiazepines. Vitals showed HR 65, BP 122/71, RR 18, temp $97.9^{\circ} \mathrm{F}$. Results of CBC, CMP, EtOH, ASA, and APAP were unremarkable.

Results: The patient became more drowsy with $\mathrm{SpO}_{2}$ in the low 90 s. Naloxone infusion was started at $0.4 \mathrm{mg}$ /hour. Approximately 24 hours after reported use, opioid intoxication symptoms resolved. Whole blood levels of 2-Methyl AP-237, measured by liquid chromatography tandem mass spectrometry, peaked at $35 \mathrm{ng} / \mathrm{mL}$ approximately seven hours after nasal administration. Designer benzodiazepines were also detected, including pyrazolam $(4200 \mathrm{ng} / \mathrm{mL})$, clonazolam $(63 \mathrm{ng} / \mathrm{mL})$, and etizolam. Other drugs detected included methadone, mitragynine, Odesmethyltramadol, and eutylone.

Conclusion: This patient presented with symptoms of opioid overdose following nasal atomization of 2-Methyl AP-237 with concomitant use of benzodiazepines and methadone. This case highlights the emerging public health threat associated with designer opioid use, combination with benzodiazepines, and overdose.

093. Emergency Department Utilization Patterns in Patients with Opioid Related Emergency Department Visits

Siri Shastry ${ }^{1}$, Evan Shegog ${ }^{2}$, George Loo ${ }^{1}$, Ethan Cowan ${ }^{1}$

${ }^{1}$ Icahn School of Medicine at Mount Sinai, New York, NY, USA.

${ }^{2}$ McGovern Medical School, Houston, TX, USA

Background: Opioid-related Emergency Department (ED) visits have surged over the past decade but there is limited data on ED utilization patterns of patients with opioid use disorder (OUD). An improved understanding may underscore missed opportunities for screening, intervention and referral. We seek to more thoroughly investigate ED utilization patterns in this population.

Methods: This was a retrospective matched case-control study at a single urban ED. Cases were patients with an opioid-related index
ED visit from June 1, 2017, to May 31, 2018. Controls were patients with a non-opioid-related index ED visit from June 1, 2018, to May 31, 2019. Cases and controls were matched 2:1 on age and gender. The primary outcome was the association between the number of ED visits in the 24-month period surrounding the index visit (12 months prior and 12 months following) and case status. Cochran-Mantel-Haenszel statistics and conditional logistic regression were employed using SAS University Edition v9.4.

Results: There were a total of 224 cases. The mean case age was 43.9 (95\% CI: $42.1,45.7)$ and $76.8 \%$ were male. Cases had a mean of 1.80 $(1.32,2.28)$ visits preceding and $2.18(1.48,2.88)$ visits following the index visit. Controls had a mean of $1.00(0.75,1.25)$ visits preceding and $0.78(0.34,1.12)$ visits following the index visit. More than one ED visit in the 24-month period surrounding the index visit was significantly associated with odds of being a case patient (OR: 4.407, CI: 2.866, 6.775).

Conclusion: In this study, patients with an opioid-related index visit had higher rates of ED utilization preceding and following the index visit when compared to an age and gender matched control population. These findings suggest that there are significant opportunities for ED intervention and referral to outpatient treatment prior to and following an opioid-related ED visit in this patient population.

094. What Did You Say? A Case-Series of Opioid-Induced Acute Sensorineural Hearing Loss in an Urban Emergency Department in the Upper Midwest

Zachary A Davidson, Travis D Olives

Hennepin Healthcare, Minneapolis, MN, USA

Background: Opioid-induced sensorineural hearing loss (SNHL) has been described in the medical literature since the early 2000 s. While the exact pathophysiology remains unknown, proposed mechanisms include hypoxia, direct ototoxicity, and kappa opioid receptor overstimulation.

Methods: This is a case series of three Emergency Department (ED) patient encounters during August-September 2020 at an urban tertiary care center in the upper midwest. Patient 1: A $25 \mathrm{M}$ with intermittent drug use who reported smoking an "M30" was found apneic with an $\mathrm{SpO} 2$ of $40 \%$ by emergency medical services (EMS). He aroused to naloxone and reported acute hearing loss. Despite spontaneous resolution of SNHL, he received oral steroids in the ED, and saw otolaryngology (ENT) and audiology at discharge. Patient 2: $51 \mathrm{M}$ with chronic substance abuse history called EMS complaining of hearing loss. EMS noted pinpoint pupils and hypoxia, both improved with naloxone. He required written communication, and was admitted and assessed by ENT and audiology. His hearing improved without steroids. Patient 3: A 29M who reported taking an "M30" was found down, and improved after naloxone. On admission, he complained of tinnitus and hearing loss; hearing loss gradually improved, but tinnitus persisted until discharge. He did not receive audiology testing.

Results: All patients admitted street opioid use, two of whom endorsed taking M-30s, a locally common fentanyl-containing counterfeit opioid tablet. All three patients endorsed hearing loss, and two demonstrated mild-moderate SNHL on follow-up audiogram and a normal ear exam. One patient endorsed only tinnitus, with a grossly normal hearing exam. The patient with most severe deficits received a brain MRI, without evidence of ischemia. Confirmatory urine toxicology testing was positive for fentanyl and clozapine.

Conclusion: Opioid-induced hearing loss is an under-recognized phenomenon. Emergency providers should be aware of opioid-induced ototoxicity and be prepared to diagnose, treat, and ensure urgent follow-up for affected patients. 
095. Epidemiology of Exposures Involving Opioids and Alcohol Using a National Poison Database

Saumitra Rege, Heather Borek, Aaron Frey, Christopher Holstege University of Virginia, Charlottesville, VA, USA

Background: The aim of the current study was to evaluate the epidemiology of exposures reporting coexisting opioid and alcohol to the U.S. Poison Centers (PCs).

Methods: The National Poison Data System (NPDS) was queried for exposures reporting both opioids and alcohol from 2011 to 2018. Cases resulting in major medical outcomes or deaths were categorized as serious medical outcomes (SMO). We descriptively assessed the demographic and clinical characteristics of exposures. Temporal trends were evaluated using generalized linear mixed models. Independent predictors of SMO were studied using logistic regression.

Results: There were 48,127 exposure calls involving opioids and alcohol during the study period. The frequency decreased by $29.5 \%(p<0.001)$, and the rate decreased by $21.2 \%(p<0.001)$. There were 1,112 deaths in our study sample (2.3\%), with $9.1 \%$ of cases demonstrating major effects. Among cases with SMO, there was a greater proportion of cases demonstrating the presence of additional substances $(72.8 \%$ vs. $64.1 \%)$ as compared to non-SMO exposures. The risk of SMO was the highest in cases over 60 years of age (Ref: 20-29 years) (AOR: 1.99, 95\% CI: 1.75-2.26). Males were $15 \%$ more likely than females to have serious exposures to opioids and alcohol (AOR: 1.15, 95\% CI: 1.08-1.22). In cases involving substances in addition to opioids and alcohol, the risk of SMO increased 2 -fold. Other important predictors of an SMO were suspected suicides (Ref: Unintentional exposure) (AOR: 1.74, 95\% CI: 1.57-1.92), parenteral route of administration (Ref: Ingestion) (AOR: 3.85, 95\% CI: 3.014.94) and exposure to benzodiazepines (Ref: no benzodiazepines) (AOR: $1.10,95 \%$ CI: $1.02-1.18)$.

Conclusion: Analysis of calls to U.S. PCs indicated a decreasing trend of reported exposures involving coexisting alcohol and opioids. Exposures resulting in SMO demonstrated a high risk among intentional reasons for exposures and occurred in older age groups.

096. NMDA-Receptor Antagonist Cross-Tolerance From Chronic Dextromethorphan Misuse Rendering Prehospital Ketamine Ineffective for Agitation: a Case Report

Michelle M Nguyen ${ }^{1}$, Jon B Cole ${ }^{1,2}$, Paul C Nystrom ${ }^{1}$, Travis D Olives $^{2,1}$, Ann M Arens ${ }^{2,1}$

${ }^{1}$ Hennepin Healthcare, Minneapolis, MN, USA. ${ }^{2}$ Minnesota Poison Control System, Minneapolis, MN, USA

Background: Dextromethorphan is commonly found in antitussive medications and has potential for recreational abuse. In supratherapeutic doses, the active metabolite dextrorphan antagonizes NMDA receptors, which produces dissociative, "out-of-body" experiences, similar to ketamine, which is frequently used by prehospital and emergency providers to treat profound agitation.

Hypothesis: Chronic recreational use of dextromethorphan may result in cross-tolerance to other NMDA-antagonists, resulting in higher doses of NMDA antagonists needed for parenteral sedation of agitated patients.

Methods: This is a single-patient chart review. This is a 33-year-old man with dextromethorphan use disorder and recurrent emergency department (ED) evaluations for dextromethorphan toxicity (163/174 of hospital encounters over 110 months). In this case, he was found agitated and combative by prehospital providers and received $500 \mathrm{mg}$ ketamine IM for agitation, which had no clinical effect. On arrival, the patient remained significantly agitated. He improved after midazolam seven $\mathrm{mg}$ IV and droperidol $20 \mathrm{mg}$ IV, without any adverse effects. Laboratory studies were notable for hyperchloremia (134 $\mathrm{mEq} / \mathrm{L}$ ). After six hours of observation, the patient returned to his baseline mental status and was discharged.
Results: This case describes an acutely agitated patient with chronic dextromethorphan use who did not respond as expected to a dose of ketamine for sedation. We performed a literature review using PubMed with keywords "dextromethorphan," "ketamine," "tolerance," and "NMDA antagonist," finding no reports of resistance to ketamine in patients with chronic dextromethorphan abuse. This case exemplifies the importance of physiologic tolerance, likely due to up-regulation of NMDA receptors, and the need for either higher dosing of NMDA antagonists or using non-NMDA antagonists to treat agitation.

Conclusion: Chronic use of dextromethorphan may lead to NMDAreceptor upregulation and cross-tolerance to other NMDA-antagonists such as ketamine. When such patients develop agitation necessitating parenteral medications, benzodiazepines or antipsychotics may be preferable.

097. Withdrawal Seizures in a Patient Dependent on the Novel Benzodiazepine Pyrazolam

Nhunguyen Le ${ }^{1}$, Fiona Garlich ${ }^{1,2}$

${ }^{1}$ LAC+USC Medical Center, Los Angeles, CA, USA. ${ }^{2}$ Keck School of Medicine, University of Southern California, Los Angeles, CA, USA

Background: Pyrazolam is a novel designer benzodiazepine that can be purchased online as a "research chemical" for recreational and selfmedication purposes. Structurally similar to alprazolam, pyrazolam is typically used for anxiolysis with users reporting less sedation and euphoria than with other benzodiazepines. We present a case of withdrawal seizures in a patient with pyrazolam dependence.

Hypothesis: Severe withdrawal from novel benzodiazepines may go unrecognized due to lack of clinician familiarity and absence of significant withdrawal signs.

Methods: This is a single-patient chart review. A 24-year-old male who had been taking pyrazolam $25 \mathrm{mg}$ bid for over one year presented to the Emergency Department (ED) reporting a seizure several hours prior to arrival. Four days prior to presentation, his pyrazolam shipment did not arrive, and he began feeling shaky, dizzy, and generally unwell. On the day of presentation, he had visited an outside ED two times, the second after having a witnessed seizure at home. Both times he was discharged without receiving medications, with suspicion for drug-seeking behavior. Results: On arrival, vital signs were temperature $36.8 \mathrm{C}^{\circ}$, heart rate 74 / $\mathrm{min}$, respiratory rate $16 / \mathrm{min}$, blood pressure $125 / 77 \mathrm{mmHg}$, and oxygen saturation $100 \%$. He was alert and non-toxic appearing without gross tremor. Close examination revealed dilated pupils, fine hand tremor, and $4+$ deep tendon reflexes. The patient had a one minute generalized tonic-clonic seizure in the ED and was treated contemporaneously with benzodiazepines, followed by intravenous phenobarbital and an oral phenobarbital taper. He was discharged after 24 hours observation without recurrent seizure.

Conclusions: Withdrawal seizures in patients dependent on the novel benzodiazepine pyrazolam may occur in the absence of classic signs of withdrawal such as tachycardia or gross tremor. Lack of clinician familiarity with this emerging drug class may lead to under-recognition and undertreatment of withdrawal symptoms.

\section{Pyrazolam Ingestion Leading to Profound CNS Depression}

William J Trautman, Carin K Malley, Matthew P Scanlon, Rachael C Westover, Andrew F Micciche, Joshua A Shulman, Michael G Abesamis University of Pittsburgh Medical Center, Pittsburgh, PA, USA

Background: Limited knowledge exists about 'designer benzodiazepines' despite increased availability over the past few years. Pyrazolam is a novel benzodiazepine with some structural homology to alprazolam. Little is known about its pharmacokinetics. 
Hypothesis: Pyrazolam ingestion can result in a severe sedative-hypnotic toxidrome.

Methods: This is a single-patient case report. A 24-year-old male presented to an emergency department (ED) with profound CNS depression. Naloxone was administered without improvement in his status, and he ultimately required intubation for airway protection. He was transferred to a tertiary care center for unknown substance ingestion. The patient's initial exam was notable for constricted, poorly reactive pupils, minimal gag reflex, and trace extremity movement to painful stimuli only off sedation. Laboratory evaluation via enzyme multiplied immunoassay technique (EMIT) urine drug screening was positive for amphetamine/ methamphetamine, benzodiazepines, cocaine, and marijuana. His alcohol level was $42 \mathrm{mg} / \mathrm{dL}$, blood glucose $58 \mathrm{mg} / \mathrm{dL}$, and his routine labs were within normal limits.

Results: Liquid chromatography-mass spectrometry urine testing revealed pyrazolam, amphetamine, methamphetamine, cocaine, levamisole, caffeine, naloxone, and nicotine. No other benzodiazepines or sedatives were detected. The patient required 36 hours of intubation secondary to lack protective airway reflexes and intermittent apnea. After extubation, he endorsed stimulant use but denied intentional benzodiazepine use. He acknowledged drinking from the glass of a friend who is known to use benzodiazepines recreationally.

Conclusion: Pyrazolam is a novel benzodiazepine with limited information on its effects in the medical literature. While the unknown ingested dose prevents us from determining quantitative pharmacokinetics, this case suggests that pyrazolam can cause profound central nervous system depression with a relatively longer duration of action than other structurally homologous benzodiazepines.

099. Severe Sedative and Alcohol Dependence Treated With Ambulatory Detox Using Mix of Telehealth and In-Person Visits During COVID-19.

Timothy J. Wiegand ${ }^{1}$, Mayra Rodriguez ${ }^{2}$

${ }^{I}$ Department of Emergency Medicine, University of Rochester School of Medicine \& Dentistry, Rochester, NY, USA. ${ }^{2}$ Huther Doyle, Rochester, NY, USA

Background: Resources for detoxification treatment have been limited during COVID-19.

Hypothesis: Alcohol and sedative dependence can be successfully managed using ambulatory detoxification protocols.

Methods: Single-patient case report. A 54-year-old male with alcohol and sedative use disorder, sober for 6 months, started drinking, "during COVID-19," three pints vodka nightly and twelve $24 \mathrm{oz}$ malt liquor beers/day, with two mg lorazepam 3 times/day, "to stop the shakes," at work. He initially entered detox, but left 36 hours later, and was rapidly back drinking. After agreeing to consents, wife holding medications, and mix of video/on-site evaluations naltrexone $50 \mathrm{mg} \mathrm{PO} /$ day, gabapentin $900 \mathrm{mg} \mathrm{PO} / \mathrm{QID}$ (+ dose at night PRN), clonidine $0.1 \mathrm{mg}$ PO QID PRN + $0.1 \mathrm{mg}$ patch/week, and valproic acid $500 \mathrm{mg}$ PO BID were prescribed. He received $40 \mathrm{mg}$ diazepam at detoxification but was anxious, shaky, and mildly tachycardic until starting gabapentin and VPA, when his symptoms improved. Five days of QID gabapentin changed to TID and VPA was tapered to $250 \mathrm{mg}$ BID at two weeks, dc'd at four. XR-naltrexone was initiated after week one. Initial EtG and EtS were + two days after starting ambulatory detox but negative after. Visits included telemedicine (audio-video) days one, three, five, and on-site on day two and four, with Zoom ${ }^{\circledR}$ group/ provider meetings biweekly. No further drinking occurred confirmed by toxicology and other assessment.

Results: Ambulatory detoxification protocols are increasingly important as COVID-19 disrupts traditional models of care. Alcohol and other drug use has increased dramatically during the pandemic, along with the complications (withdrawal, DWIs, domestic violence). Clinicians need to be familiar with telehealth protocols for ambulatory detoxification and the medication regimens used in ambulatory detoxification.

Conclusion: Alcohol and other drug use has dramatically increased during COVID-19. Understanding protocols and guidelines to treat dependence and withdrawal in ambulatory settings are critical.

\section{Severe Gamma-Butyrolactone Withdrawal Treated with Baclofen and Phenobarbital}

Garret A Winkler, Sam T Ontiveros, Alicia B Minns, Daniel R Lasoff University of California San Diego, San Diego, CA, USA

Background: Gamma-Butyrolactone (GBL) is a GABA-B agonist and is a recreational drug with popularity among the men who have sex with men patient population. GABA-B agonist withdrawal is reported to be difficult to control and baclofen use has been reported for management of mild withdrawal symptoms.

Hypothesis: Oral baclofen and phenobarbital can be used to treat severe GBL withdrawal syndromes.

Methods: This is a case report of one patient treated with baclofen and phenobarbital for GBL withdrawal. A 27-year-old man with history of daily methamphetamine use, infrequent ethanol use, and TID GBL use presented from a mental health facility with confusion. The initial emergency department (ED) visit was due to tachycardia, diaphoresis, hypertension, and hallucinations. The patient was treated with $780 \mathrm{mg}$ phenobarbital IV with resolution of his symptoms and on discharge CIWA was two. The patient represented that evening with tachycardia, diaphoresis, hypertension, agitation, altered mental status, and hallucinations. Vital signs were HR 118 bpm, BP 117/70 mmHg, temp $99.8 \mathrm{~F}$, and RR 18.

Results: The patient was administered $520 \mathrm{mg}$ phenobarbital IV, $2 \mathrm{mg}$ lorazepam IV, and $10 \mathrm{mg}$ baclofen PO. Withdrawal symptoms were controlled in the ED and the patient was admitted for withdrawal syndrome management and rhabdomyolysis. Baclofen was titrated from $10 \mathrm{mg}$ TID to $20 \mathrm{mg}$ TID over hospital days 4 to 6 due to continued withdrawal symptoms. Phenobarbital was initially stopped during his hospitalization and restarted due to worsening withdrawal symptoms. Both baclofen and phenobarbital were used as PRN therapy for severe, difficult to control symptoms. The symptoms were controlled on baclofen $20 \mathrm{mg}$ PO TID and phenobarbital $130 \mathrm{mg}$ PO BID, and the patient was discharged to mental health on hospital day 10 when asymptomatic.

Conclusion: Baclofen with adjunctive phenobarbital should be considered a treatment for gamma-butyrolactone withdrawal and GABA-B withdrawal syndrome.

\section{Cardiac Arrest and Prolonged Coma Following Overdose of Veterinary Euthanasia Solution}

Andrew F Micciche, Rachael C Westover, Matthew P Scanlon, Carin K Malley, Michael E Bux, Ouwen Yang, Douglas D Rockacy, Michael J Lynch

University of Pittsburgh Medical Center, Pittsburgh, PA, USA

Background: Multiple medications exist for use in veterinary euthanasia and are likely unfamiliar to emergency providers. Individuals with access to these agents may use them intentionally to commit suicide.

Methods: This is a single-patient case report. A 21-year-old female veterinary technician alerted family she planned to overdose. Upon paramedic arrival, she was found to be pulseless but had return of spontaneous circulation following two minutes of cardiopulmonary resuscitation. She was intubated upon arrival to the emergency department and started on epinephrine for refractory hypotension. Laboratory studies showed a metabolic acidosis $(\mathrm{pH} 7.17$, bicarbonate $15 \mathrm{mMol} / \mathrm{L}$ ) with a lactate of 6.8 $\mathrm{mMol} / \mathrm{L}$ and ethanol level of $9 \mathrm{mg} / \mathrm{dL}$. Paramedics brought two large 
syringes with remnants of a pink solution from the scene, raising concern that she had ingested a euthanasia solution containing pentobarbital (390 $\mathrm{mg} / \mathrm{mL})$, phenytoin $(50 \mathrm{mg} / \mathrm{mL})$, ethanol, and propylene glycol. Exposure to this solution was confirmed when the patient's phenytoin level returned at $7.21 \mathrm{mcg} / \mathrm{mL}$. Urine drug screen was positive for barbiturates and marijuana. She was given one dose of activated charcoal due to presence of euthanasia solution in gastric aspirate.

Results: Initial pentobarbital level was $37 \mathrm{mcg} / \mathrm{mL}$. Given the relatively short half-life of pentobarbital, multi-dose activated charcoal and urinary alkalinization were not pursued. Pentobarbital level 36 hours following presentation increased to $40 \mathrm{mcg} / \mathrm{mL}$ but subsequently decreased to 17 $\mathrm{mcg} / \mathrm{mL} 48$ hours after the second measurement. The patient was comatose without need for sedation for the first four days and was extubated on hospital day six without neurologic deficit. She endorsed overdosing on $50 \mathrm{~mL}$ of the aforementioned euthanasia solution.

Conclusion: A high index of suspicion for euthanasia agents is prudent when veterinary personnel present with an overdose. In excess, these agents may cause cardiovascular collapse, cardiac arrest, and prolonged coma, with duration of effects being difficult to predict.

\section{Airway Obstruction and Cardiac Arrest in a Cocaine Stuffer} Hassan AL-Balushi, ${ }^{1,2}$, Andres Guzman-Soto ${ }^{1,2}$, Kyle Suen ${ }^{1,2}$, Ziad Kazzi $^{1,2}$, Jonathan de Olano ${ }^{1,2}$, Todd Taylor ${ }^{1,2}$

${ }^{1}$ Emory University School of Medicine, Department of Emergency Medicine, Division of Medical Toxicology, Atlanta, Georgia, USA. ${ }^{2}$ Georgia Poison Center, Atlanta, Georgia, USA

Background: Body stuffing is defined as ingesting small quantities of drugs in poorly sealed packets often to avoid repercussions from law enforcement. Cocaine is one of the drugs most commonly involved. Complications reported with stuffing include aspiration, esophageal obstruction and fatal toxicity. Mechanical airway obstruction has not been reported.

Methods: A 32-year-old male presented to the Emergency Department with police after stuffing a cocaine packet. He developed agitated delirium, and despite administration of $10 \mathrm{mg}$ midazolam and $20 \mathrm{mg}$ haloperidol intramuscular, he required intubation. Once intubated, he was noted to have mydriasis, hypertension and ECG changes of regular wide complex tachycardia (QRS: $240 \mathrm{~ms}$ ), right bundle-branch block pattern and a terminal $\mathrm{R}$ wave in AVR. He was started on sodium bicarbonate until QRS interval normalized. Over the following hours, he was noted to have a high peak pressure and was difficult to ventilate with a bag-valve mask. He was treated with salbutamol, paralytics, and ketamine without response. Chest radiograph was unremarkable. An emergent bronchoscopy was performed, revealing a plastic bag at the distal end of the endotracheal tube. Attempt to retrieve the bag was complicated by total airway obstruction and cardiac arrest. Return of spontaneous circulation was achieved after temporizing left main intubation and resuscitation. Emergency tracheostomy was performed and the plastic bag containing a white powder was removed.

Results: The patient's hospital course was complicated by rhabdomyolysis, elevated liver enzymes, and acute kidney injury requiring dialysis. He was discharged in stable condition after four weeks.

Conclusion: We present a cocaine body stuffer complicated by agitated delirium, cardiotoxicity and airway obstruction. Haloperidol may worsen sodium channel blockade in the setting of acute cocaine toxicity. Airway obstruction should be considered a hazard in body stuffers, particularly in the setting of elevated peak airway pressures after intubation.

103. Massive Clonidine Overdose Leading to Sustained Bradycardia, Hypertension, and PRES

Rowan H Kelner ${ }^{1}$, Rebecca Pilkerton ${ }^{1}$, Alyrene Dorey ${ }^{1,2}$
${ }^{1}$ University of Utah, Salt Lake City, Utah, USA. ${ }^{2}$ Utah Poison Control Center, Salt Lake City, Utah, USA

Background: Clonidine is a centrally acting alpha-2 agonist, typically associated with transient hypertension followed by hypotension and bradycardia in overdose. The proposed mechanism of hypertension in clonidine overdose is loss of alpha- 2 selectivity at high serum concentrations. We report a case of clonidine overdose resulting in sustained bradycardia and hypertension followed by seizure and posterior reversible encephalopathy syndrome (PRES).

Methods: This is a single-patient case report. A 15-year-old female with a past medical history of depression presented to the emergency department one hour after ingestion of 247 tablets of Clonidine $\mathrm{HCl} 100 \mathrm{mcg}$. The patient was initially asymptomatic but over subsequent hours developed bradycardia (HR $43 \mathrm{bpm}$ ) and severe hypertension (BP 180/110 $\mathrm{mmHg}$ ) followed by deteriorating mental status. A nitroprusside infusion was initiated and naloxone $10 \mathrm{mg}$ was administered. Shortly thereafter, the patient suffered a convulsion and was intubated and sedated. A stat MRI showed cortical FLAIR hyperintensity within the posterior circulation compatible with PRES. Activated charcoal was given and whole bowel irrigation performed. The patient required nitroprusside for blood pressure control for approximately 48 hours; hypotension never developed. On hospital day five, the patient was extubated. Following extubation, the patient experienced persistent AMS which slowly improved. She was ultimately discharged to psychiatric care.

Results: National Medical Services toxicology screen (1876SP) obtained 43 hours after ingestion revealed a clonidine level of $19 \mathrm{ng} / \mathrm{mL}$ (therapeutic range, $0.2-2.0 \mathrm{ng} / \mathrm{ml}$ ).

Conclusion: This case is the first, to our knowledge, of clonidine monoingestion causing sustained hypertension and PRES. This case emphasizes the need to pursue aggressive treatment of hypertension in the setting of clonidine overdose. Use of short-acting infusion agents may be preferable to mitigate the risk of developing subsequent hypotension.

\section{Role of High-Dose Folic Acid in Methanol Toxicity}

Matthew P Scanlon ${ }^{1}$, Ryan T Marino ${ }^{2}$, Alexander M Sidlak ${ }^{3}$

${ }^{1}$ University of Pittsburgh School of Medicine, Pittsburgh, PA, USA.

${ }^{2}$ University Hospitals, Cleveland, OH, USA. ${ }^{3}$ Inova Fairfax, Great Falls, VA, USA

Background: Folic acid has been proposed as an adjunct to alcohol dehydrogenase inhibitors and hemodialysis (HD) in methanol toxicity. Though no clinical studies show efficacy, the risks of folic acid are minimal and animal evidence shows a benefit in expediting the clearance of formate.

Hypothesis: Folic acid administration decreases toxicity and shortens the duration of toxicity for patients with methanol toxicity.

Methods: We performed a retrospective chart review from 2012 to 2018 of patients with methanol concentrations $>10 \mathrm{mg} / \mathrm{dL}$. Patients receiving scheduled dosing of $50 \mathrm{mg}$ of folic acid or leucovorin (FG) were compared to those either receiving no adjunctive therapy or sub-therapeutic dosing of folic acid (NFG). Patients not requiring hospitalization, those with alternate causes of acidosis, or those who died prior to therapy were excluded. The primary outcome measures were worsening metabolic acidosis, length of stay (LOS), and serious adverse outcomes. Half-life of methanol was also recorded.

Results: Out of 27 patients identified with a positive methanol level, 14 were included (Nine in FG and five in NFG). Initial pH and methanol level were similar between the FG and NGF groups (7.29 vs. 7.26 and 118 vs. $120 \mathrm{mg} / \mathrm{dL}$ ). No difference in LOS was detected (3.3 d [IQR: 1.9 7.2] vs. $3.0 \mathrm{~d}$ [2.8-3.9]). No patients had worsening metabolic acidosis during therapy. In patients treated without and with $\mathrm{HD}$, median half-life of methanol in FG vs. NFG was similar (2748 m [range: 1428-2997] vs. $2368 \mathrm{~m}$ [1945-2791]; $216 \mathrm{~m}$ [159-879 m] vs. $191 \mathrm{~m}$ [186-506]). 
Conclusion: Folic acid treatment provided no additional benefit to standard therapy for methanol toxicity. As expected, there was no difference in the half-life of methanol with folic acid treatment. We cannot rule out the benefit of an initial dose of folic acid while formate levels are elevated.

105. Trend of Ethanol Intoxication and Withdrawal During the COVID-19 Quarantine in the Einstein Healthcare System

Richard J Chen, James A Krueger

Einstein Healthcare Network, Philadelphia, PA, USA

Background: COVID-19 pandemic resulted in a quarantine period from March-June 2020, which resulted in liquor store closures in Pennsylvania. This restricted access to alcoholic beverages to online orders, but because many people worked from home, there is a reported increase in ethanol consumption. The resulting impact on presentations of ethanol intoxication and ethanol withdrawal to the hospital is not clear.

Hypothesis: Due to quarantine, there will be both an increase in diagnoses of ethanol withdrawal and increase in cases of ethanol intoxication.

Methods: This is a retrospective study of cases of ethanol intoxication and withdrawal presenting to three hospitals of Einstein Healthcare Network from January to June 2019 and 2020. Reports were generated using ICD10 diagnoses via Cerner.

Results: There were a total 3000 cases of intoxication in 2019 and 2630 cases in 2020, representing a decrease of $12 \%$. Cases were $67 \%$ male in both 2019 and 2020. Cases of intoxication decreased in March-June 2020 from 2019, with a low of 332 cases in April 2020 (38\% decrease). Cases were majority African American (59.3\% and 55.9\% for 2019 and 2020, respectively) followed by Caucasian ( $23.2 \%$ for both years). There were 48 cases $(81 \%$ male) of ethanol withdrawal in 2019 and 80 cases $(71 \%$ male) in 2020. Except in May, the cases in 2020 were greater each month than in 2019. There was no statistical difference in age for either year for withdrawal or intoxication.

Conclusion: From 2019 to 2020, there was an increase in the cases of ethanol withdrawal presenting to the hospital, while cases of intoxication were lower during March-June 2020. Decrease in access to ethanol and overall lower utilization of the ER during quarantine were likely contributing factors. This data provides some insight into the impact that the pandemic has had on ethanol use in Philadelphia.

\section{Severe Methanol Toxicity During COVID-19}

Abdullatif Aloumi ${ }^{1}$, Abbas A M T Altamimi ${ }^{2}$, Abdulaziz Alsarraf ${ }^{3}$, Robert J Hoffman

${ }^{1}$ Sidra Medicine, Qatar Poison Center, Doha, Qatar. ${ }^{2}$ Amiri Hospital, Emergency Department, Kuwait City, Kuwait. ${ }^{3}$ Adan Hospital, ICU, Kuwait City, Kuwait

Background: During the COVID-19 pandemic, alcohol consumption has increased along with difficulty in obtaining alcoholic beverages. Both intentional and unintentional ingestion of methanol may result when alcohol consumers are unable to procure ethanol and instead ingest alternatives for inebriant effects.

Hypothesis: The null hypothesis is the incidence of severe methanol toxicity did not increase during COVID-19.

Methods: This is a retrospective study of consecutive patients presenting to a single intensive care unit (ICU) of a tertiary academic medical center which serves as the main hospital for the capital of the country. The electronic medical record (EMR) of the ICU was reviewed to identify all patients admitted with severe methanol toxicity from April 2019 to December 2020. The local health department case definition of severe methanol toxicity was the following: anion gap metabolic acidemia; noncomatose with associated visual disturbance or comatose; requiring dialysis; history of recent alcohol use; and no plausible alternative diagnosis.
Three medical toxicologists blinded to the date of exposure reviewed the cases to confirm the diagnosis of severe methanol toxicity with no plausible alternative diagnosis.

Results: Ten patients with severe methanol toxicity were identified. Two were in the pre-COVID-19 time frame (April 2019-December 2019), and eight patients during COVID-19 (April 2020-December 2020). In this sample, the relative risk of severe methanol toxicity during COVID-19 was 4.0 , which is a $400 \%$ increase relative to the pre-COVID time frame. Conclusion: In the location of this study, where ethanol is commercially unavailable, an increase in severe methanol toxicity was noted. In circumstances when alternatives to ethanol are likely to be ingested, clinicians should be vigilant to detect toxicity from methanol and possibly other substances.

\section{Serum Electrolytes Changes in Long Term Alcohol Misusers}

Kyaw Soe Htun, Ko Ko Win, Zaw Min Tun, Arkar Aung, Ye Lin Aung, Thein Zaw, Tin Maung Hlaing

Defence Services Medical Research Centre, Tatkone, Naypyitaw, Myanmar

Background: In long-term use of alcohol, the toxic effect of acetaldehyde which damage the cell membranes results in generalized reduction in reabsorptive ability of the proximal tubular cells of kidney, alteration of fluid balance, serum electrolyte and acid-base abnormalities. In routine practice, testing electrolytes is not included in medical investigations normally. This study aimed to explore the early assessment for risk of long-term alcohol misusers by detection of serum electrolytes such as sodium, potassium, chloride, calcium and magnesium.

Methods: The study was conducted on 43 long-term misusers with normal liver function tests compared with 43 control participants after obtaining informed consent. Serum sodium, potassium and chloride were estimated by easylyte electrolyte analyzer. Serum calcium and magnesium were done by biochemical analyzer cobas 311 .

Results: The mean values ( \pm standard deviation) of sodium, potassium, chloride, calcium and magnesium in cases were $136.39 \pm 6.21,3.5 \pm 0.58$, $105 \pm 7.49,9 \pm 0.84$ and $1.86 \pm 0.37$, respectively, while it was $140 \pm$ $3.42,4.08 \pm 0.45,100 \pm 2.82,9.40 \pm 0.18$ and $1.90 \pm 0.18$ in controls. Electrolyte changes such as hyponatremia (44\%), hypokalaemia (34\%), hyperchloremia $(58 \%)$, hypocalcemia $(23 \%)$ and hypomagnesaemia $(25 \%)$ were observed in long-term misusers.

Conclusion: Checking electrolytes in routine medical care particularly to long-term alcohol misusers is highly recommended. Out of testing, early recognition of all these changes is of paramount importance for their appropriate management to lessen morbidity and mortality in such patients as the proximal tubular defect is reversible by discontinuation of alcohol using.

\section{Alcoholic Ketoacidosis: Analysis of Current Management}

Alexander M Sidlak ${ }^{1}$, William Trautman ${ }^{2}$, Ryan T Marino ${ }^{3}$, Michael J Lynch $^{4}$

${ }^{I}$ Inova Fairfax, Falls Church, VA, USA. ${ }^{2}$ University of Pittsburgh Medical School, Pittsburgh, PA, USA. ${ }^{3}$ University Hospitals, Cleveland, OH, USA. ${ }^{4}$ University of Pittsburgh Medical School, Pittsburgh, PA, USA

Background: Alcoholic Ketoacidosis (AKA) is a common complication from alcohol use characterized by an elevated anion gap metabolic acidosis (AGMA) in patients with a recent history of heavy alcohol use. Though the underlying pathophysiology is well characterized, no studies on the efficacy of thiamine and limited evidence on the ideal treatment regimen exist. Therefore, we sought to analyze the current treatments utilized in patients with isolated AKA. 
Methods: We performed a one-year retrospective review of patients with isolated AKA complicated by metabolic derangements. Baseline characteristics, laboratory abnormalities, and treatments were recorded. We also performed a regression analysis between the timing of treatment administration and time to metabolic improvement.

Results: Thirty episodes of AKA were identified. Administration of thiamine and dextrose occurred $3.5 \mathrm{~h}$ and $4.3 \mathrm{~h}$ after presentation, respectively. Nearly all patients received both thiamine, dextrose, and crystalloid infusions with a single patient only receiving dextrose, and two only receiving thiamine. Time to thiamine and dextrose correlated with time to improvement, but time to crystalloid did not. Independent factors associated with earlier improvement included time to dextrose and early treatment $(<3.5 \mathrm{~h})$ with thiamine.

Conclusion: Alcoholic ketoacidosis can produce potentially severe metabolic derangements. Early treatment with dextrose and thiamine may lead to earlier improvement while delay can result in an extended duration of metabolic abnormalities.

109. Pilot Study of Methylene Blue as an Antidote for Amlodipine Poisoning in Mice

Lisandra de Castro Brás, Cecile L. Baccanale, Lex Eccleston, Trey Sloan, Jason C. Antoine, Steven Verzwyvelt, Peggy Pittman, Dorcas O'Rourke, William J. Meggs

East Carolina University, Geenville, NC, USA

Background: Methylene blue has been proposed as an antidote for refractory shock from amlodipine poisoning because of its vasoconstrictive effects as a nitric oxide scavenger.

Hypothesis: Methylene blue reduces cardiovascular toxicity in amlodipine overdose.

Methods: A randomized controlled study in mice was conducted. Twelve C57Bl/6 mice were poisoned with amlodipine $90 \mathrm{mg} / \mathrm{kg}$ by oral gavage and then anesthetized with isoflurane. Mice were randomized to methylene blue $20 \mathrm{mg} / \mathrm{kg}$ in $5 \%$ dextrose by intra-peritoneal injection, or an equal volume of $5 \%$ dextrose. Serial cardiovascular measures included blood pressure, pulse, and central venous pressure. Ultrasound was used to determine cardiac output, contractility, and left ventricular dimensions. Blood pressure readings were obtained with a non-invasive tail-cuff system (SC1000, Hatteras Instruments). Central venous pressure was measured with Doppler ultrasound. Survival to two hours was recorded. Data analysis used Grubbs method to identify outliers with an alpha of 0.05 . The Grubbs method was followed by an Unpaired $T$-test. $p<0.05$ was considered significant.

Results: Two hour survival rate was $83 \%$ for methylene blue-treated animals, while only $50 \%$ of the controls survived to two hours, which did not obtain statistical significance. The methylene blue group displayed higher ejection fraction (EF) after two hours compared to vehicle controls (vehicle $\mathrm{EF}=14.4 \pm 15.4$, methylene blue $\mathrm{EF}=43.4 \pm 18.7$, $p=0.007)$. There was a significant difference in heart rate at the endpoint between treated and control animals. The methylene blue treatment group displayed increased LV fractional shortening (FS) compared to vehicle controls (vehicle $\mathrm{FS}=4.5 \pm 3.2$, methylene blue $\mathrm{FS}=11$ g. $0 \pm 4.9, p=$ 0.029 ). Methylene blue treatment blunted the decline in cardiac output $(\mathrm{CO})$ : vehicle $\mathrm{CO}=2.9 \pm 4.1$, methylene blue $\mathrm{CO}=11.2 \pm 5.6, p=0.027$ ). Conclusion: This pilot study found efficacy of methylene blue for amlodipine overdose in mice.

110. Percutaneous Left Ventricular Assist Device (Impella) for Fatal Refractory Calcium Channel Blocker Toxicity: a Case Report

Jonathan Schimmel ${ }^{1}$, Sasha Kaiser ${ }^{2,3}$, Janetta L. Iwanicki ${ }^{2,3}$

${ }^{1}$ Dept of Emergency Medicine, Division of Medical Toxicology, Mount Sinai Hospital Icahn School of Medicine, New York, NY, USA. ${ }^{2}$ Dept of
Emergency Medicine, Denver Health and Hospital Authority, Denver, CO, USA. ${ }^{3}$ Rocky Mountain Poison \& Drug Safety, Denver Health and Hospital Authority, Denver, CO, USA

Background: Calcium channel blocker (CCB) overdose management has shifted toward high-dose insulin and vasopressors; however, refractory cases may require mechanical circulatory support. Information is limited on percutaneous left ventricular assist devices (pLVAD) (Impella ${ }^{\circledR}$ ) for drug-induced shock, and they are not addressed by $\mathrm{CCB}$ toxicity guidelines.

Research Question: The objective was to describe pLVAD risks and benefits in a case of drug-induced refractory shock.

Methods: This is a case report from a tertiary-care hospital without ECMO. A healthy 30 -year-old female presented with normal vital signs after ingesting verapamil 3.6 grams. Deterioration occurred 24 minutes later with BP 49/26 and HR 61. The patient received activated charcoal, intravenous fluid, atropine, calcium salts, glucagon, high-dose vasopressors, high-dose euglycemic insulin therapy, and a bicarbonate drip and dialysis for metabolic acidosis. Transvenous pacing for atrioventricular block achieved capture after repositioning.

Results: An Impella $\mathrm{CP} \circledast \mathrm{pLVAD}$ was placed 15 hours post-ingestion via the left common femoral artery, requiring multiple re-positionings for difficulty obtaining sufficient flow without suction, and was successful toward the apex. Pre-placement cardiac output and cardiac index were reduced. Post-placement mean arterial pressure (MAP) increased to 70s. The pLVAD flow rate was repeatedly adjusted due to suction alarms, and decreased flow rates were associated with decreased MAP. Echocardiography showed pLVAD inlet migration. Red blood cells were transfused when hemoglobin decreased from 16.1 to $5.4 \mathrm{~g} / \mathrm{dL}$. The patient died 36 hours post-ingestion. Initial serum verapamil was $1,400 \mathrm{ng} / \mathrm{mL}$. Conclusion: Mixed cardiogenic and distributive shock were likely present. Frequent pLVAD suction alarms are due to poor positioning or preload, and, in this case, was likely from distal positioning. The pLVAD likely caused hemolysis. High flow rates were associated with increased MAP, though it was unable to prevent death. This case highlights that although there is potential benefit, positioning is complex with numerous risks.

111. Lacosamide Overdose with Significant QRS Widening Reversed by Sodium Bicarbonate

Jorge L Castaneda ${ }^{1}$, Meghan B Spyres ${ }^{1,2}$, Zubair Ahmed ${ }^{2}$, Steven C Curry ${ }^{1,2}$

${ }^{I}$ Banner University Medical Center Phoenix, Phoenix, AZ, USA. ${ }^{2}$ University of Arizona Medical School Phoenix, Phoenix, AZ, USA

Background: Lacosamide exerts its antiepileptic effect via neuronal sodium channel inhibition. Lacosamide induced cardiotoxicity secondary to sodium channel blockade and treatment response to sodium bicarbonate is not well described.

Hypothesis: Lacosamide induced cardiotoxicity can be effectively reversed with sodium bicarbonate $(\mathrm{NaHCO} 3)$.

Methods: This is a single-patient chart review. A 54-year-old man with a history of seizure disorder overdosed on lacosamide. He presented with seizure-like activity and BP 155/85 mmHg, HR 103 bpm and labs significant for a mild anion gap metabolic acidosis $\left(\mathrm{pH} 7.296, \mathrm{HCO}_{3} 18 \mathrm{meq} / \mathrm{L}\right.$, AG 18), $\mathrm{Na}^{+} 139 \mathrm{meq} / \mathrm{L}, \mathrm{K}^{+} 3.5 \mathrm{meq} / \mathrm{L}, \mathrm{BUN} 26 \mathrm{mg} / \mathrm{dl}, \mathrm{sCr} 1.9 \mathrm{mg} / \mathrm{dL}$, and lactic acid $6.4 \mathrm{mmol} / \mathrm{L}$. ECG showed sinus rhythm, PR $294 \mathrm{~ms}$, terminal R aVR of $3 \mathrm{~mm}$, QRS $106 \mathrm{~ms}$, and QTc $480 \mathrm{~ms}$. NaHCO3 infusion was started at $15 \mathrm{meq} / \mathrm{hr}$ due to concern for sodium channel toxicity. On day 2 of hospitalization, $\mathrm{NaHCO} 3$ infusion was stopped given no further QRS widening. One hour later, he developed bradycardia (HR $45 \mathrm{bpm}$ ) and widened QRS to $160 \mathrm{~ms}$. Fifty meq $\mathrm{NaHCO} 3 \times 2$ was given with improvement in HR to $65-80 \mathrm{bpm}$. Twenty minutes later, bradycardia recurred (HR $45 \mathrm{bpm}$ ), again responsive to $\mathrm{NaHCO} 3$ bolus (HR 50-80 bpm and QRS 102) and a NaHCO3 drip at $50 \mathrm{meq} / \mathrm{hr}$ was 
started. No significant dysrhythmias recurred. QRS on discharge was baseline $90 \mathrm{~ms}$. Urine GCMS from presentation detected lacosamide, diphenhydramine, and levetiracetam. Diphenhydramine level drawn on initial labs was below plasma detection level.

Results: The lacosamide level on presentation was $97 \mathrm{mg} / \mathrm{L}$ (therapeutic range $4-12 \mathrm{mg} / \mathrm{mL}$ ). Terminal elimination kinetics were calculated using seven plasma lacosamide levels, revealing first-order kinetics and a terminal elimination half-life of 24.7 hours.

Conclusion: $\mathrm{NaHCO} 3$ effectively reversed lacosamide-induced cardiotoxicity. Elimination half-life kinetics after this large overdose were longer than typically reported.

\section{Iatrogenic Supratherapeutic Digoxin Administration in a} Neonate Treated with Digoxin Immune FAB Fragments

\author{
Joshua R McFalls ${ }^{1,2}$, Deven S Bhatia ${ }^{2}$, Kapil Sharma ${ }^{1}$ \\ ${ }^{1}$ University of Texas Southwesetern Medical Center, Dallas, TX, USA. \\ ${ }^{2}$ Parkland Memorial Hospital, Dallas, TX, USA
}

Background: Digoxin is a common medication used in the treatment of congestive heart failure and arrhythmias. There are no reports of digoxin toxicity being treated with digoxin immune Fab fragment (DIF) in neonates.

Hypothesis: Treatment of digoxin toxicity with DIF in the neonatal population is safe.

Methods: This is a case report. A newborn was delivered at 37 weeks by spontaneous vaginal delivery with no complications. On day five of life, the patient started to have supraventricular tachycardia (SVT). SVT was not broken with ice to the face or adenosine treatment. Cardiology was consulted and recommended digoxin treatment with an initial dose of $15 \mathrm{mcg} / \mathrm{kg}$. However, the patient was given $50 \mathrm{mcg} / \mathrm{kg}$ in error $(150 \mathrm{mcg})$. Total digoxin level 3.5 hours after administration was $8.35 \mathrm{ng} / \mathrm{mL}$ with a capillary potassium of $5.5 \mathrm{mmol} / \mathrm{L}$ and normal renal function. The patient had no ectopy on EKG or rhythm strip. Toxicology was consulted and recommend DIF treatment.

Results: Because of the predictable binding pattern of DIF, a partial reversal of digoxin was attempted to bring the digoxin dose to a therapeutic level. The patient was given $1 / 5$ of a vial of DIF to bind $100 \mathrm{mcg}$ of digoxin. Free digoxin level after DIF administration was $4.0 \mathrm{ng} / \mathrm{mL}$ (normal range $0.8-2.0 \mathrm{ng} / \mathrm{mL}$ ) nine hours after digoxin administration. The patient remained asymptomatic during the next seven days of hospital stay with stable potassium levels. No repeat doses of digoxin were required. Many challenges to this case exist. Digoxin levels could have been falsely elevated due to digoxin-like substances. Potassium levels are difficult to interpret with capillary samples. Pharmacokinetic data of DIF-digoxin complexes are uncertain in neonates.

Conclusion: DIF use in neonates appears safe. This case illustrates a few of the challenges associated with treatment in this age group.

113. Successful Treatment of Vasopressor and High-Dose Insulin Resistant Shock from Poly-drug Cardiotoxic Overdose with Methylene Blue, Angiotensin II, and ECMO

Christopher N Sweat ${ }^{1,2}$, Alexandru Ulici ${ }^{1}$, Carrie L Oakland ${ }^{1}$, Jon B Cole $^{1,3}$

${ }^{1}$ Minnesota Poison Control System, Minneapolis, MN, USA. ${ }^{2}$ Regions Hospital, Saint Paul, MN, USA. ${ }^{3}$ Hennepin Healthcare, Minneapolis, MN, USA

Background: Ingestion of cardioactive medications continues to be a common cause of poisoning mortality, causing both distributive and cardiogenic shock. Multiple rescue therapies have been proposed.
Hypothesis: The combination of methylene blue, angiotensin II, and ECMO may successfully overcome cardiotoxic shock from poly-drug overdose resistant to vasopressors and high-dose insulin (HDI).

Methods: This is a case report of an 18-year-old female presenting to the ED six hours after ingesting $300 \mathrm{mg}$ amlodipine and 30 tabs each of unknown strength carvedilol, furosemide, and $25 \mathrm{mg}$ hydrochlorothiazide. She presented hypotensive and tachycardic and received two grams calcium gluconate and two liters crystalloid with brief improvement to BP 93/56, HR 120. She was intubated and underwent gastric lavage with removal of residual pill fragments, then started on vasopressors due to worsening shock. At 20 hours, post ingestion lactate was rising $(4.1 \mathrm{mmol} / \mathrm{L})$ and she was requiring HDI at nine units $/ \mathrm{kg} /$ hour, norepinephrine $0.45 \mathrm{mcg} / \mathrm{kg} /$ min, vasopressin 2.4 units $/ \mathrm{min}$, angiotensin II $40 \mathrm{ng} / \mathrm{kg} / \mathrm{min}$, and phenylephrine to maintain MAPs $>50 \mathrm{mmHg}$. This was likely due to vasoplegia as echocardiography demonstrated EF of $65-70 \%$ with concomitant pulse of $130 \mathrm{bpm}$. Propofol was discontinued and the patient was administered methylene blue bolus $(1 \mathrm{mg} / \mathrm{kg})$ and infusion ( $2 \mathrm{mg} / \mathrm{kg} /$ hour $)$ as a nitrous oxide scavenger, with transient improvement in MAP to $>60 \mathrm{mmHg}$. Ultimately, she was cannulated for VA ECMO given rising lactate $(7.1 \mathrm{mmol} / \mathrm{L})$ and recurrent shock.

Results: Following methylene blue and ECMO, she achieved a cardiac output of 4.5 liters/min without further decompensation. She was then weaned off vasopressors on hospital day five, then ultimately decannulated HD7 and extubated HD9 with good neurologic outcome. Conclusion: The combination of high-dose insulin, vasopressors, methylene blue, angiotensin II, and VA-ECMO successfully resuscitated cardiogenic and distributive shock from poly-drug cardiotoxic overdose.

\section{Esmolol for Flecainide Overdose in an Infant}

Stephanie L Widmer, Wells Brambl, Joshua Nogar Northwell Health, Manhasset, NY, USA

Background: Flecainide is a class IC antidysrhythmic agent which acts by selectively blocking fast inward sodium channels and is used to treat supraventricular tachycardias. All formulations of flecainide exhibit a narrow therapeutic window and a high bioavailability of about $90 \%$.

Methods: A 5-month-old male with a history of Wolff-Parkinson White (WPW) presented to the emergency department (ED) two hours after a 10 -fold dosing error $(80 \mathrm{mg}$ instead of $8 \mathrm{mg}$ ). Patient was clinically normal upon initial presentation. At three hours post-ingestion, he was noted to have wide complex dysrhythmia on telemetry. He was treated with sodium bicarbonate $10 \mathrm{mEq} \times 4$, with transient narrowing after each administration. He also received $2 \%$ lidocaine $1 \mathrm{mg} / \mathrm{kg} \times 2$, NS bolus of $150 \mathrm{cc}$ and intralipid therapy $1.5 \mathrm{cc} / \mathrm{kg}$ with no improvement. Patient remained in wide complex dysthymia and was taken to the pediatric ICU for possible ECMO initiation. Patient had an episode of hypoxia to $84 \%$ and a respiratory rate increase to the $40 \mathrm{~s}$ with rising end tidal CO2. $\mathrm{He}$ was subsequently intubated after receiving etomidate $0.3 \mathrm{mg} / \mathrm{kg}$, rocuronium $1 \mathrm{mg} / \mathrm{kg}$, sodium bicarb $1 \mathrm{mEq} / \mathrm{kg} \times 3$, and adenosine $\times 2$ $(0.1 \mathrm{mg} / \mathrm{kg}$ and $0.2 \mathrm{mg} / \mathrm{kg})$. The patient briefly converted to sinus rhythm after each adenosine administration. During intubation, he converted to sinus rhythm and an esmolol infusion $(50 \mathrm{mcg} / \mathrm{kg} / \mathrm{min})$ was initiated. $\mathrm{He}$ remained in sinus rhythm for the rest of his hospital stay. On day two, the patient was extubated and transitioned to propranolol and amiodarone, which he will continue upon discharge. The flecainide level at six hours post-ingestion was $0.93 \mathrm{mcg} / \mathrm{mL}(0.20-0.99 \mathrm{mcg} / \mathrm{mL}$ reference range).

Conclusion: Esmolol slows the rate of discharge of the sinoatrial node and increases the functional refractory period of the atrioventricular node, which may be useful properties in mitigating wide complex dysrhythmias caused by flecainide. 
115. PERM Syndrome: a Potential Mimic of Glycine Antagonist Poisoning

Matthew P Scanlon, Carin K Malley, Rachael C Westover, Andrew F Micciche, Michael G Abesamis, Joshua A Shulman

University of Pittsburgh Medical Center, Pittsburgh, PA, USA

Background: Progressive encephalomyelitis with rigidity and myoclonus (PERM) syndrome is an uncommon neurologic condition characterized by autonomic instability and severe, spastic muscular contractions. The condition is typically linked to anti-glycine receptor (anti-GlyR) autoantibodies that develop in the context of underlying autoimmunity or paraneoplastic disease.

Hypothesis: De novo diagnoses of PERM syndrome may be clinically indistinguishable from glycine antagonist poisoning.

Methods: This is a single-patient case report. A six-year-old fully immunized girl with a history of sexual abuse was transferred to a tertiary care dedicated pediatric facility for evaluation of "spells" typified by generalized shaking and rigidity. The patient was reportedly awake and in obvious distress during these episodes, which were characterized by pronounced muscle contractions of the extremities and arching of the back. The patient's laboratory assessment demonstrated sequelae of rhabdomyolysis with a creatine phosphokinase (CPK) of 38,000 IU/L. Medical toxicology was consulted with concern for possible glycine antagonist poisoning.

Results: On examination, the patient had findings consistent with opisthotonos. The patient had no clear exposure to rodenticidal agents or penetrating injuries prior to her presentation. Pending completion of her diagnostic workup, the patient was treated empirically with tetanus immunoglobulin, metronidazole, and benzodiazepines with minimal improvement in her symptoms. Subsequent MRI of the brain and tetanus antibodies returned within normal limits. Liquid chromatography-mass spectrometry showed no evidence of strychnine exposure. Given concern for possible autoimmune disease, the patient was started on IVIg therapy with a notable decrease in both the frequency and severity of her spasms. Prior to discharge, autoimmune titers confirmed the presence of antiGlyR autoantibodies.

Conclusion: PERM syndrome represents a rare glycinopathy that may mimic glycine antagonist toxicity and should be considered in the differential of patients presenting with opisthotonos, neuromuscular spasticity, and rhabdomyolysis.

116. Prolonged Serotonin Syndrome in the Setting of Fulminant Hepatic Failure with Subsequent Living-Donor Liver Transplantation

Carin K Malley, Matthew P Scanlon, Rachael C Westover, Andrew F Micciche, Emily B Brant, Anthony F Pizon, Joshua A Shulman

University of Pittsburgh Medical Center, Pittsburgh, PA, USA

Background: Serotonin syndrome is characterized by hyperreflexia, myoclonus, confusion, diaphoresis, tachycardia, and hyperthermia. Under normal conditions, serotonin syndrome resolves rapidly, usually within 24-48 hours. Little is known about the toxicodynamics of serotonergic medications in the setting of fulminant hepatic failure and liver transplantation.

Hypothesis: Serotonin syndrome due to hepatically metabolized xenobiotics will be prolonged in the setting of fulminant hepatic failure.

Methods: This is a single-patient case report. A 33-year-old female presented at least 12 hours after an intentional ingestion of acetaminophen, duloxetine, hydroxyzine, linaclotide, and dicyclomine. Her initial acetaminophen level was $>600$. She was promptly started on Nacetylcysteine and was transferred to a quaternary care center. Initial examination revealed hyperreflexia, sustained clonus, and increased muscle tone in the lower extremities consistent with serotonin syndrome, likely secondary to synergistic toxicity from hydroxyzine and duloxetine.
Results: Despite N-acetylcysteine, fomepizole, and extracorporeal removal of acetaminophen, the patient progressed to fulminant liver failure and underwent living donor liver transplantation on post-ingestion day four. Post transplantation, the patient continued to exhibit sustained clonus and encephalopathy despite an appropriately sized and functioning liver transplant. Repeat liquid chromatography mass spectrometry quantitative time of flight (LCMS-QTOF) obtained on urine on post ingestion day eight continued to show duloxetine and hydroxyzine. Her mental status and clonus gradually improved and resolved by post-ingestion day 11.

Conclusion: Supermassive ingestions often cause persistent symptoms despite shorter medication half-lives. In this case, duloxetine, with a halflife of 12 hours, demonstrated continued serotonergic effects for 10 days post ingestion in the setting of fulminant liver failure with subsequent liver transplantation. This duration is substantially longer than expected, even in large ingestions. Thus, toxicity from hepatically metabolized xenobiotics may be prolonged in newly transplanted living-donor livers.

\section{Intentional Ingestion of Isobutyl Nitrite Causing Nearly Fatal Methemoglobinemia: Case Report}

Kerollos A. Shaker, Kapil Sharma

University of Texas Southwestern, Dallas, TX, USA

Background: Inhalational isobutyl nitrite abuse, commonly known as poppers, is used for euphoric and sexual stimulatory purposes. It causes relaxation of the smooth muscles, vasodilation, and tachycardia. Oral ingestion can be very dangerous and lead to life-threatening hypotension and methemoglobinemia.

Methods: This is a case report about a patient who presented to the Emergency Department (ED) in respiratory failure with cyanosis, hypotension, and coma. Examination of the patient and toxicology consultation occurred at bedside in the ED and during the hospitalization. Management of the methemoglobinemia occurred with toxicology guidance.

Results: Patient is a 56-year-old male with past medical history of hypertension, gastro-esophageal reflux disease, and alcohol use disorder who presented to the ED with altered mental status, cyanosis, respiratory failure, and hypotension. He had mistakenly ingested rather than inhaled $10 \mathrm{~mL}$ of isobutyl nitrite while intoxicated at a nightclub. On presentation to the ED, he had desaturations to $60 \%$ and hypotensive BP $86 / 47 \mathrm{mmHg}$ requiring norepinephrine and vasopressin infusions. His blood had a chocolate-brown blood appearance. He had methemoglobin levels $>31 \%$ and improved with three doses of methemoglobinemia (total receiving $2 \mathrm{mg} / \mathrm{kg}$ ) and improved to a methemoglobin level of $0.7 \%$. Patient developed green urine following methylene blue administration. Patient did not have evidence of hemolysis on labs. He was ultimately extubated the third day of hospitalization and discharged.

Conclusion: Accidental oral ingestion of isobutyl nitrite has shown to be dangerous in leading to hemodynamic instability and methemoglobinemia due to its vasodilatory and oxidizing effects, respectively.

\section{Resolution of Severe Sodium Nitrite-Induced Methemoglobinemia} with Single Dose Methylene Blue

Patrick C Filkins ${ }^{1,2}$, Brett L DeBruyn ${ }^{1}$, Melissa H Gittinger ${ }^{1,3}$

${ }^{1}$ Georgia Poison Center, Atlanta, Georgia, USA. ${ }^{2}$ Grady Health System, Atlanta, Georgia, USA. ${ }^{3}$ Emory University School of Medicine, Atlanta, Georgia, USA

Background: Nitrites are potent oxidizing agents commonly found in well water, food, industrial compounds, and pharmaceuticals known to induce methemoglobinemia. Sodium nitrite is a readily available form 
that is easily purchased online and its use for self-harm is well documented.

Hypothesis: A conservative dosing strategy could be sufficient in treating greatly elevated methemoglobin levels.

Methods: This is a single-patient case report. A 17-year-old female with a past medical history of depression presented to the emergency department hypoxic, hypotensive, and minimally responsive. Initial vitals were HR 133 bpm, BP $72 / 44$ mmHg, RR 25, $\mathrm{SPO}_{2} 87 \%$ on a non-rebreather, and GCS of three. The patient was intubated, and norepinephrine was initiated. A venous blood gas (VBG) revealed a methemoglobin level of $84.8 \%$ and oxyhemoglobin of $6.9 \%$. The recommendation was made to administer methylene blue $1 \mathrm{mg} / \mathrm{kg}$ with serial VBGs to reassess the response.

Results: Methylene blue one $\mathrm{mg} / \mathrm{kg}$ was administered intravenously over 30 minutes. Repeat cooximetry analysis at one and three hours post infusion showed a decrease in methemoglobin levels to $17.4 \%$ and $3.9 \%$ respectively. Nine hours post-infusion, the methemoglobin level had normalized to $<0.1 \%$. Mental status and oxygenation had returned to baseline levels, norepinephrine was titrated off, and the patient was extubated to room air. The patient confirmed she ingested approximately two-thirds of a 113-gram bottle of sodium nitrite which was purchased online in a suicide attempt.

Conclusion: Providers should be aware of such readily accessible toxic agents. Sodium nitrite is a relatively inexpensive agent that can be easily purchased via online retailers and result in significant toxicity. We report one of the highest reported survived methemoglobin levels at $84.8 \%$. Low-dose methylene blue at an initial dose of one $\mathrm{mg} / \mathrm{kg}$ may be appropriate even in severe cases of methemoglobinemia improving resource utilization and avoiding adverse reactions.

\section{Flumazenil Continuous Infusion to Treat Novel Liquid Benzodiazepine Flubromazolam Intoxication}

Helen W Hou, Colgan Sloan, Alyrene A Dorey

University of Utah Health, Salt Lake City, Utah, USA

Background: Flubromazolam is a long-acting, synthetic benzodiazepine (SBZD) with higher potency than its structurally related benzodiazepine compounds. There is limited data on the efficacy of flumazenil to reverse flubromazolam intoxication.

Hypothesis: Repeated doses of flumazenil followed by continuous flumazenil infusion can reverse sedation and respiratory depression from flubromazolam acute intoxication.

Methods: This is a case report of a 22-year-old woman who presented to the emergency department after reportedly ingesting about $15 \mathrm{~mL}$ of liquid "clonazolam $1.17 \mathrm{mg} / \mathrm{mL}$ " she had purchased from the Internet in a suicide attempt. One hour after ingestion, she experienced worsening somnolence and respiratory depression, and received a total of $0.3 \mathrm{mg}$ flumazenil intravenous boluses $(0.1 \mathrm{mg}$ followed by $0.2 \mathrm{mg}$ one hour later) followed by $0.25 \mathrm{mg} / \mathrm{hr}$ continuous infusion for eight hours. Serum non-synthetic benzodiazepine (NBZD) immunoassay and a quantitative screen were performed within 24 hours of ingestion. Drug, serum, and urine samples were also sent to the National Medical Services (NMS) Laboratories to detect the presence of SBZD via high-resolution mass spectrometry.

Results: Neither the benzodiazepine detection immunoassay nor quantitative benzodiazepine screen detected the presence of NBZD and their metabolites in the patients' samples. The expanded SBZD screen performed by NMS later revealed the presence of flubromazolam and metizolam in the drug sample instead of clonazolam. Only flubromazolam was detected in the patient's serum and urine samples. After each flumazenil bolus, the patient improved in wakefulness. An infusion was initiated due to the short duration of effect of flumazenil and the patient's anticipated clinical course. No adverse events occurred. After the infusion, the patient did not experience further respiratory depression but reported "feeling out of it" for several days after her ingestion.

Conclusion: Low-dose intravenous flumazenil boluses followed by continuous infusion can effectively reverse the sedative effects of flubromazolam intoxication without producing adverse effects.

\section{Lead Poisoning Complicated by a Lead Particulate Appendiceal Impaction.}

Vincent J Calleo, Jonathan D Hund, Michael J Hodgman

SUNY Upstate Medical University, Syracuse, NY, USA

Background: Management of lead poisoning with a gastrointestinal burden includes bowel cleansing prior to chelation. We present a unique case of appendiceal lead particulate accumulation following whole-bowel irrigation (WBI) necessitating an appendectomy.

Methods: A 41-year-old male was referred to toxicology clinic with one year of malaise, amnesia, and abdominal pain. His venous blood lead was $120 \mu \mathrm{g} / \mathrm{dL}$. The patient reported lead dust inhalation while renovating an old building without proper personal protective equipment. There was no reported oral lead exposure so succimer was initiated based on inhalational history. Three weeks after succimer initiation, he developed worsening abdominal pain and was admitted to the hospital. His lead level was $133 \mu \mathrm{g} / \mathrm{dL}$. Radiology's CT abdomen pelvis interpretation was no acute pathology. Toxicology's CT review noted numerous particulate hyperdensities suspicious for lead. WBI was initiated. Serial radiographs demonstrated accumulation of lead particulates in the right lower quadrant with CT confirmation of appendiceal lead impaction. Surgical consultants recommended an endoscopic washout prior to surgical management; the washout was unsuccessful and a laparoscopic appendectomy was successfully performed. His lead level decreased markedly postoperatively even prior to initiating chelation with succimer and calcium disodium EDTA. A repeat lead level upon completion of EDTA was 15 $\mu \mathrm{g} / \mathrm{dL}$. He was discharged on the remaining succimer but was unfortunately lost to follow-up.

Results: While medical literature has described lead pellet impactions of the appendix, descriptions of appendiceal lead particulate accumulation after initiating WBI are lacking. Surgery was understandably hesitant to perform an appendectomy since surgical intervention for appendiceal lead particulate impaction has not yet been described.

Conclusion: Lead source identification can be challenging and imaging prior to chelation should be considered. An appendectomy followed by chelation dramatically lowered our patient's blood lead concentration after an appendiceal impaction with lead particulates.

\section{Fomepizole for Severe Disulfiram Reaction Complicated by} Shock

Alexander M Sidlak ${ }^{1}$, Amy M Ziegler ${ }^{2}$, Michael J Lynch ${ }^{2}$

${ }^{1}$ Inova Fairfax, Great Falls, VA, USA. ${ }^{2}$ University of Pittsburgh School of Medicine, Pittsburgh, PA, USA

Background: Disulfiram reactions are uncommonly encountered from disulfiram given the decreased utilization of this agent for alcohol use disorder. While reactions are often self-limited and brief, they can be severe and lead to shock.

Hypothesis: Fomepizole may mitigate the severity and duration of a disulfiram reaction.

Methods: A 41-year-old man on chronic disulfiram (over two months) as treatment for his alcohol use disorder presented symptomatic after drinking $\sim 750 \mathrm{~mL}$ of liquor in an attempt to harm himself. He was hypotensive $(78 / 37 \mathrm{mmHg}$ ), flushed, and hypoxemic ( $84 \%$ oxygen saturation on room air) requiring $100 \%$ Fi02 by non-rebreather. Despite 2 L IV $0.9 \% \mathrm{NaCl}$, he remained hypotensive (mean arterial pressure (MAP): $51 \mathrm{mmHg}$ ). 
Following unsuccessful volume resuscitation, $0.3 \mathrm{mg}$ subcutaneous epinephrine, $20 \mathrm{mg}$ IV diphenhydramine, and $20 \mathrm{mg}$ IV famotidine were given to treat possible anaphylaxis. Chest radiograph was normal. Ethanol level was $80 \mathrm{mg} / \mathrm{dL}$. Despite these interventions, the patient's condition did not improve appreciably; therefore, a $15 \mathrm{mg} / \mathrm{kg}$ dose of fomepizole was given. After administration of fomepizole, blood pressure and heart rate normalized, and the patient was weaned to room air over the next hour. Acetaldehyde was detectable, but not quantifiable. Ethanol was metabolized at a rate of nine $\mathrm{mg} / \mathrm{dl} / \mathrm{h}$. The patient was discharged after a night of observation.

Results: By inhibiting alcohol dehydrogenase, fomepizole theoretically prevents further accumulation of acetaldehyde, the molecule responsible for the clinical effects seen in a disulfiram reaction. Though these effects are often transient, in severe cases, adjunctive use of fomepizole may be warranted.

Conclusion: Fomepizole can be an effective adjunctive therapeutic in patients with severe disulfiram reactions and in whom ethanol is still present.

122. Fomepizole as Adjunctive Therapy for Delayed Acute Acetaminophen Overdose and Repeated Supratherapeutic Ingestion

Sasha K Kaiser ${ }^{1}$, Richard C Dart ${ }^{1}$, Christopher Hoyte ${ }^{1,2}$

${ }^{I}$ Rocky Mountain Poison and Drug Safety, Denver, Colorado, USA.

${ }^{2}$ Department of Emergency Medicine, University of Colorado, Denver, Colorado, USA

Background: Acetaminophen (APAP) overdose is a common cause of acute liver failure worldwide. N-Acetylcysteine (NAC) is the only current antidote approved for clinical use for acetaminophen hepatotoxicity. While most cases respond to NAC therapy, patients presenting with delay to care or repeated supratherapeutic ingestion (RSTI) can develop acute liver failure and death despite NAC administration. Fomepizole as a CYP2E1 inhibitor may have a potential role in mitigating acetaminophen related liver injury by reducing early oxidative metabolism and late mitochondrial oxidative stress.

Methods: Four patients presented to local tertiary care hospitals after APAP overdose. A single dose of $15 \mathrm{mg} / \mathrm{kg}$ fomepizole was administered to two patients presenting with RSTI with AT products greater than 10,000, and one patient with delayed acute ingestion with AT product greater than 10,000. One additional patient with chronic acetaminophen use, severely prolonged acetaminophen half-life and large AT product was also treated.

Results: All patients improved following fomepizole infusion and survived to hospital discharge. The case series includes a 30 -year-old $\mathrm{F}$ who presented 31 hours after intentional acute acetaminophen ingestion with AT product of 31,020. Additionally, a 46-year-old M presented hypotensive, with signs of multi-organ dysfunction following RSTI. His initial AT product was 90,252. Third, a 44-year-old M with RSTI ingestion presented with an initial AT product of 231,763. Finally, a 54-year-old $\mathrm{M}$ with chronic acetaminophen presented with an initial AT product of 39,592.

Conclusion: We report a series of fomepizole-treated patients with the highest reported initial alanine transaminase, and another with the highest AT product. Each patient had multiple markers on presentation predicting poor outcomes; however, all had surprising smooth clinical courses. Fomepizole administration may be beneficial even in the setting of significantly elevated transaminases or delay to care. Further research is needed.

123. High Dose Insulin Therapy for Inotropic Support During Venoarterial Extracorporeal Membrane Oxygenation Decannulation

Kartik R Shah, Thomas M Przybysz, Deepu Ushakumari, Ann-Jeannette Geib

Atrium Health's Carolinas Medical Center, Charlotte, NC, USA
Background: High-dose insulin (HDI) therapy has been used as inotropic support for toxin-induced cardiogenic shock, but literature suggests that it can also be used in non-toxin-induced cardiogenic shock states. Its use has not been reported in veno-arterial extracorporeal membrane oxygenation (VA-ECMO) decannulation.

Methods: Single-patient chart review. A 56-year-old male presented with acute biventricular nonischemic cardiac failure without reported toxic ingestion. He was supported with continuous renal replacement therapy, percutaneous left ventricular (LV) assist device, and VA-ECMO. After eight days of support, he was planned for VA-ECMO decannulation. Results: During the decannulation, he was monitored with transesophageal echocardiography (TEE). Initially, LV ejection fraction (EF) was estimated at $10-15 \%$. Regular insulin bolus $1 \mathrm{U} / \mathrm{kg}$ with 25 grams of dextrose was given 20 minutes prior to decannulation, and $1 \mathrm{U} / \mathrm{kg} /$ hour insulin infusion was started. TEE prior to decannulation showed LVEF 30-40\%. Transthoracic echocardiography (TTE) 3.5 hours after HDI bolus revealed normal LV systolic function; LVEF 50-55\%. HDI infusion continued for 24 hours after the bolus. A dextrose infusion was started five hours after HDI bolus with rates of $0.33-0.5 \mathrm{~g} / \mathrm{kg} /$ hour. His potassium was $3.6 \mathrm{mEq} / \mathrm{L}$ before HDI bolus; subsequently, his nadir was $3.2 \mathrm{mEq} / \mathrm{L}$. He received $30 \mathrm{mmol} / \mathrm{L}$ in total of potassium-phosphate before insulin infusion was stopped. After the insulin infusion was stopped, the dextrose infusion continued for 24 hours; a total additional $30 \mathrm{mmol} / \mathrm{L}$ of potassium-phosphate was given. He was discharged 26 days after decannulation. Repeat TTE six months later showed LVEF 15-20\% but he had resumed activities, including cycling 10 miles/day.

Conclusion: While multiple interventions occurred during decannulation, HDI therapy may have assisted in transitioning off ECMO support, and HDI should be investigated as an adjunctive option in future decannulations and other non-toxin-induced cardiogenic shock states.

\section{Octreotide Dosing and Route of Administration in Sulfonylurea Toxicity}

Erin Ryan ${ }^{1}$, William Rushton ${ }^{2}$

${ }^{1}$ Alabama Poison Information Center, Birmingham, AL, USA.

${ }^{2}$ University of Alabama at Birmingham, Birmingham, AL, USA

Background: Octreotide is commonly used to treat hypoglycemia in the setting of sulfonylurea poisoning, but optimal dosing for this indication is not well defined. The goal of this project is to review the efficacy of various octreotide dosing and routes.

Methods: A literature review was performed of octreotide use in sulfonylurea poisoning. The keywords sulfonylurea and octreotide were used to search PubMed and abstracts from toxicology conferences (North American Congress of Clinical Toxicology, European Association of Poison Control Centres and Clinical Toxicologists, and American College of Medical Toxicology Annual Scientific Meeting) to identify relevant case reports. Cases were excluded if there was no information on octreotide dosing.

Results: Fifty-five patients from 36 references were identified. Thirtytwo unique octreotide regimens were used ( 20 adult [age $>12$ years], 12 pediatric), differing in dose, interval, and/or route. Most patients $(76.4 \%)$ received multiple doses (range: $2-7$ doses) or continuous infusions, with dosing intervals ranging from 6 to 12 hours (subcutaneous [SQ]: 6-12 hours, intravenous [IV]: 6-9 hours). Individual doses ranged from 25 to $100 \mathrm{mcg}$ (SQ: $25-100 \mathrm{mcg}$, IV: $50-100 \mathrm{mcg}$ ) in adults (94.4\% between 50 and $100 \mathrm{mcg}$ ) and $0.9-2.5 \mathrm{mcg} / \mathrm{kg}$ (SQ: $0.9-2 \mathrm{mcg} / \mathrm{kg}$, IV: $1-2.5$ $\mathrm{mcg} / \mathrm{kg}$ ) in children. Infusion rates ranged from 25 to $125 \mathrm{mcg} / \mathrm{hr}$ in adults and $1-10 \mathrm{mcg} / \mathrm{kg} / \mathrm{hr}$ in children. In 5 cases, doses were increased or infusions started based on initial lack of efficacy. The most common route overall $(65.5 \%)$ and in adults $(80.5 \%)$ was SQ but IV bolus in children (57.1\%). In cases published between 1990 and 1999, 75\% administered 
octreotide SQ, with other routes reported with increasing frequency in subsequent decades (2000-2009: 70.6\% SQ, 2010-2019: 52.9\% SQ).

Conclusion: Dosing of octreotide in sulfonylurea toxicity varies widely, with limited consensus on the optimal dose or route of administration. Further research is needed to identify the ideal dosing strategies in this setting.

\section{Intensive Intervention to Prevent Digit Loss After Jellyfish Envenomation}

Yazeed Eldos, Robert J Hoffman

Sidra Medicine, Doha, Qatar, Qatar

Background: Jellyfish envenomations do not commonly result in serious complications. We report a case of severe skin necrosis of the fingers in a child stung by a jellyfish.

Methods: This is a single-chart case review. A previously healthy 12year-old boy was stung by a jellyfish on the left hand while in the Arabian Gulf. He immediately developed pain and swelling in the 3rd, 4th, and 5th digits. He self-treated with oral cetirizine and topical hydrocortisone for a day without improvement. Subsequently, he was referred to our tertiary pediatric emergency department. His vital signs were normal, and the affected digits were swollen, cold, dusky, and extremely tender to touch with normal capillary refill. Sensation was present and normal, no motor deficit was present, and his radial pulse was palpable. He was treated with IV medications including clindamycin $470 \mathrm{mg}$, hydrocortisone $100 \mathrm{mg}$, diphenhydramine $50 \mathrm{mg}$, acetaminophen $705 \mathrm{mg}$, and discharged on clindamycin and ibuprofen. On follow up 72 hours later, the pain had increased, and the affected digits remained swollen and dusky. A hand surgery service admitted the patient for treatment of digit ischemia. The patient was treated with alprostadil $60 \mathrm{mcg}$ IV, glyceryl trinitrate patch, and enoxaparin $47 \mathrm{mg}$ SC. Papaverine was injected into the neurovascular bundle of each involved digit.

Results: By day four of hospitalization, pain and swelling decreased. He was discharged on glyceryl trinitrate patch and oral acetaminophen. Fourteen days after the sting, sloughing of the necrotic skin was noted.

Conclusion: Pelagia sp. is commonly found in Qatar. In this case, severely diminished digit perfusion, due to vasoconstrictive, coagulation vasculopathy, and compartment syndrome are possible mechanisms of injury. Aggressive treatment was associated with sparing of all digits. Use of vasodilators, anticoagulants, and nerve blocks might improve outcome and reduce the risk of digit loss.

126. Fatal Crotaline Envenomation Resulting in Coagulopathy, Renal Failure, ARDS, and Cardiovascular Collapse Resistant to Fab and $F\left(a^{\prime}{ }^{\prime}\right)_{2}$ Antivenom

Han T Gao ${ }^{1}$, Ashley E Lock ${ }^{2}$, Shawn M Varney ${ }^{1}$, Christopher E Crane ${ }^{1}$, Lindsey Rippee ${ }^{1}$, Mark T Muir ${ }^{3}$, Lillian F Liao ${ }^{1}$

${ }^{1}$ University of Texas Health Science Center San Antonio, San Antonio, Texas, USA. ${ }^{2}$ University Health, San Antonio, Texas, USA. ${ }^{3}$ University of Texas Health Science Center San Antonio, San Antonio, Texas, USA

Background: Fatal North American Crotaline envenomations are rare and attributed to intravascular envenomation and anaphylaxis. We describe a case of envenomation resulting in cardiovascular collapse and coagulopathy refractory to $\mathrm{Fab}$ and $\left.\mathrm{F}(\mathrm{ab})_{2}\right)_{2}$ antivenom $\left(\mathrm{F}(\mathrm{ab})_{2} \mathrm{AV}\right)$.

Methods: A 67-yr-old male with hypertension and hyperlipidemia presented to the emergency department with leg pain, edema, confusion, hypotension, and tachycardia 45 minutes after rattlesnake envenomation to the right calf. Family denied prior exposure to Crotalids. He was intubated, given epinephrine, methylprednisolone, and six vials of Crotaline Fab antivenom (FabAV) without response and transported to our institution. On arrival, he received antihistamines, dexamethasone, norepinephrine, and 10 vials of $\mathrm{F}(\mathrm{ab})_{2} \mathrm{AV}$, and over the next hospital day (HD), 34 more vials of $\mathrm{F}(\mathrm{ab})_{2} \mathrm{AV}$. Initial laboratory results were platelets 285,000 / $\mathrm{mL}$, fibrinogen $113 \mathrm{mg} / \mathrm{dL}$, INR 1.8 , hemoglobin $16.2 \mathrm{~g} / \mathrm{dL}$, creatinine $1.42 \mathrm{mg} / \mathrm{dL}$, and lactate $4.3 \mathrm{mmol} / \mathrm{L}$. After the initial resuscitation and first 10 vials of $\mathrm{F}(\mathrm{ab})_{2} \mathrm{AV}$, he briefly trended towards recovery with fibrinogen $211 \mathrm{mg} / \mathrm{dL}$, INR 1.3 , creatinine $1.06 \mathrm{mg} /$ $\mathrm{dL}$, and lactate $2.2 \mathrm{mmol} / \mathrm{L}$, though platelets decreased to $210,000 /$ $\mathrm{mL}$. When HD one ended, testing showed platelets $73,000 / \mathrm{mL}$, fibrinogen $141 \mathrm{mg} / \mathrm{dL}$, INR 2.0 , and hemoglobin $6.2 \mathrm{~g} / \mathrm{dL}$ requiring blood transfusion. With the acute blood loss, worsening liver failure, and coagulopathy, 18 vials of FabAV were administered with no response. Localized swelling progressed to the abdomen, renal failure developed requiring dialysis, VV-ECMO was initiated due to ARDS, and plasmapheresis was attempted. Despite this, acidosis worsened, and he developed abdominal compartment syndrome. Family declined decompressive laparotomy for comfort measures, and the patient expired on HD four.

Conclusion: Crotaline envenomation resulting in cardiovascular collapse led to refractory coagulopathy and multisystem organ failure despite treatment for anaphylaxis and 24 vials of FabAV and 44 vials of $\mathrm{F}(\mathrm{ab})_{2} \mathrm{AV}$.

\section{Refractory Thrombocytopenia Despite Administration of Two Types of Crotalid Antivenom}

Holden Wagstaff $\mathrm{MD}^{1}$, Alyrene Dorey $\mathrm{MD}^{1,2}$

${ }^{1}$ University of Utah Division of Emergency Medicine, Salt Lake, Utah, USA. ${ }^{2}$ Utah Poison Control Center, Salt Lake, Utah, USA

Background: Several thousand crotalid envenomations occur in the United States (US) annually. Venom components may cause tissue injury, coagulopathy, thrombocytopenia, and neurotoxic effects. Venom composition varies between species of Viperidae, within a species, and within an individual viper throughout its life. Crotalid antivenom options in the US include $\mathrm{Crofab}^{\circledR}$, a Fab fragment from sheep immunized with four North American Crotalids, and Anavip ${ }^{\circledR}$, an equine F(ab)2 antivenom derived from two crotalids native to Mexico and South America. Thrombocytopenia refractory to antivenom is a rare complication of crotalid envenomation.

Methods: A healthy 50-year-old male sustained a rattlesnake envenomation to his hand while visiting Washington State. Emergency department (ED) findings included spreading redness and thrombocytopenia (nadir of platelets $20 \times 10^{3} / \mu \mathrm{L}$ ) without coagulopathy or bleeding. Control was achieved (platelets $152 \times 10^{3} / \mu \mathrm{L}$ ) with 20 vials of Crofab ${ }^{\circledR}$. The patient was discharged. Repeat studies resulting shortly after discharge showed platelets $14 \times 10^{3} / \mu \mathrm{L}$ and the patient returned to the ED. There he was treated with four vials of $C$ rofab ${ }^{\circledR}$ without a resultant change in platelet levels. Follow-up platelets 24 hours later, post-envenomation day seven, were $8 \times 10^{3} / \mu \mathrm{L}$, and four additional vials of $\mathrm{Crofab}^{\circledR}$ were ineffective. We recommended that four vials of Anavip ${ }^{\circledR}$ be administered. There was no immediate improvement in his platelet counts, which ultimately normalized on post-envenomation day 12 . There were no adverse events. The patient was diagnosed with a pulmonary embolism (PE) day 20 postenvenomation.

Conclusion: To our knowledge, this is the first attempt to control refractory thrombocytopenia by the administration of two types of crotalid antivenom. Our assumption was that antivenom produced from different crotalid species, containing different antivenom components, might better treat refractory thrombocytopenia. Though unsuccessful, there were no adverse effects from co-administration of multiple crotalid antivenoms. We suspect that his PE was provoked as he had prior provoked thrombosis. 
128. $\left.F\left(a^{\prime}\right)_{2}\right)_{2}$ Antivenom Demonstrates Lower Rates of Recurrent or Late-, New-Onset Hematologic Effects Compared to Fab Antivenom for Rattlesnake Envenomation

Danielle Nicole Mascarenas ${ }^{1}$, Lynne Fullerton ${ }^{1}$, Susan C Smolinske ${ }^{2,1}$, Brandon J Warrick ${ }^{1,2}$, Steve A Seifert ${ }^{1,2}$

${ }^{1}$ University of New Mexico Health Sciences Center, Albuquerque, NM, USA. ${ }^{2}$ New Mexico Poison Center, Albuquerque, NM, USA

Background: Our 2019 study comparing rates of persistent, recurrent, or late-new-onset (late) hematologic effects between $\mathrm{Fab}(\mathrm{CroFab} B)$ and $\mathrm{F}\left(\mathrm{ab}^{\prime}\right)_{2}$ (Anavip ${ }^{\circledR}$ ) crotalid antivenoms (AV) showed $\mathrm{F}(\mathrm{ab})_{2} \mathrm{AV}$ to have a numerical but statistically non-significantly lower rate. There were also non-significant demographic differences between groups, specifically for age, sex, and hematologic effects. The purpose of this study was to increase power by extending the previous study to include cases from the 2020 snakebite season. We hypothesized that, with larger numbers, the lower rate of late effects with $\mathrm{F}(\mathrm{ab}$ ') $2 \mathrm{AV}$ compared with FabAV would reach statistical significance and demographic differences would not.

Methods: Patients presenting with a rattlesnake bite to a healthcare facility in New Mexico between March and October 2020 were eligible for analysis in this observational study. All cases were included for patient and bite demographics. Patients with a dry bite, lost to follow-up, and those who received mixed types of antivenom or who did not receive antivenom were excluded in antivenom comparisons. The decision to treat and choice of antivenom were decided by the treating provider. Our previously published 2019 data, collected with comparable methodology, were combined with 2020 data for bivariate statistical analyses. We used Fisher's exact tests for data analysis, and a 0.05 cutoff for significance.

Results: Over the combined two bite seasons, 72 cases were included for antivenom comparison. Late effects were seen in the $\mathrm{F}(\mathrm{ab})_{2} \mathrm{AV}$ and FabAV groups in $1 / 26(4 \%)$ and $10 / 46(22 \%)$, respectively ( $p=$ .04848 ). There were no significant demographic differences, type I hypersensitivity reactions, bleeding complications, or readmissions.

Conclusion: $\mathrm{F}(\mathrm{ab})_{2} \mathrm{AV}$ results in lower rates of persistent, recurrent, or late-new-onset hematologic effects compared with FabAV in rattlesnake envenomation

\section{Abstract Withdrawn}

130. Circumferential Measurements: Hisssss-torically Discussed, Painfully Underreported

Matthew Eisenstat ${ }^{1,2}$, Joseph Carpenter ${ }^{1,2}$, Andrés Guzman-Soto ${ }^{1,2}$, Emily A Kiernan ${ }^{1,2}$

${ }^{1}$ Emory University School of Medicine, Atlanta, GA, USA. ${ }^{2}$ Georgia Poison Center, Atlanta, GA, USA

Background: Patients with progressive swelling after snake envenomation should be considered for antivenom therapy. Some references recommend objective sequential circumferential measurements to assess swelling. However, subjective swelling is frequently used as an indication to initiate antivenom therapy without limb measurements. A recent review by Ruha et al. examined snake bites reported to ToxIC database but only reported subjective limb findings. We hypothesize that the use of objective measurements of limb swelling is underutilized and may lead to inappropriate antivenom.

Methods: This is a retrospective chart review, utilizing a convenient sample of snake bites reported to a single regional poison center (RPC) from 2015 to 2019. Data was abstracted regarding whether snake envenomation occurred, snake type, whether circumferential measurements were documented, and whether antivenom was given and/or re-bolused. A random sample of 100 charts was chosen for initial analysis. Results are presented descriptively. Remaining charts are under review as part of a quality improvement project.
Results: In the study period, 2261 snake bites were reported. One hundred charts were reviewed by medical toxicologists/fellows and 13 were excluded due to incomplete data. Of the remaining 87, 46 (53\%) were cases of envenomation. Snakes identified by the caller included unknown $42(48 \%)$, copperhead 25 (29\%), non-venomous 13 (15\%), and cottonmouth seven $(8 \%)$. Circumferential measurements were obtained and documented in four (5\%) cases with no standardized method of reporting or documentation. Antivenom was given in 21 (24\%) cases and rebolused in two (2\%) cases.

Conclusion: In this RPC study, circumferential measurements were rarely performed after snake envenomation. Currently, there is no standardized method to examine/document the degree of swelling after snake envenomation. Additional research is needed to implement a standardized method to describe objective data after snake envenomations. We hypothesize that this will improve patient care, decrease inappropriate utilization of antivenom, and decrease hospital length-of-stay.

131. Evolution of Treatment for Latrodectism in a Toxicology Unit: Red-Back Spider Antivenom or Standard Analgesia? Nothing to RAVE About

Siba Sulaeman ${ }^{1}$, Andis Graudins ${ }^{2,3}$

${ }^{1}$ Monash Health, Melbourne, Victoria, Australia. ${ }^{2}$ Monash Health, Clayton, Victoria, Australia. ${ }^{3}$ Monash University, Clayton, Victoria, Australia

Background: In 2014, the Australian RAVE-2 study found that intravenous RBS-antivenom (RBSAV) was no more effective than placebo in improving pain from envenoming. We assessed practice change and responses to treatment for latrodectism in our toxicology unit, from October 2009 to June 2019.

Method: Retrospective study of RBS bite referrals. Data extracted: demographics, pain severity, treatment (oral or intravenous analgesia, RBSAV; IM or IV), response to treatment (no response or partial/ complete pain resolution), re-presentation rate, adverse events (allergic reactions, serum sickness), change in antivenom prescribing frequency.

Results: There were 252 presentations with latrodectism. Median age: 36 (range: 2-91), 46\% female. Mild pain reported in 39\%, moderate $19 \%$, and severe $38 \%$. Patients with mild pain, no initial pain score were excluded from further analysis. This left 142 cases. RBSAV was administered as initial treatment to $35 \%(n=50)$, and standard analgesic therapy in $65 \%(n=92)$. RBSAV was administered IM in $43 \%$, IV in $47 \%$, and IV/IM in $10 \%$. Median dose was one vial (range: 1-4). Those administered only analgesia received a combination of paracetamol, ibuprofen and oral/IV opioids (59.5\%), paracetamol, and ibuprofen (34\%). Ninetyone percent of patients receiving antivenom reported partial or complete reduction in pain and $94 \%$ of patients receiving analgesics had a similar response. All were discharged. There was no difference in response rate to treatment between the groups (NS). Ongoing pain resulted in representation in $6 \%(n=3)$ after RBSAV and $14 \%(n=13)$ after analgesic therapy (NS). Eighty-two percent of patients received AV from 2010 to 2013. Three instances of serum sickness and one allergic reaction (rash) to AV were detected.

Conclusion: RBSAV use declined over time in our toxicology unit, most likely attributable to practice change after the results of the RAVE-2 study. Comparison of response to treatment showed no difference in pain reduction or re-presentation rate between RBSAV and analgesia.

\section{A Case of Fatal Paralytic Shellfish Poisoning in Alaska}

Courtney Temple, Adrienne Hughes, Robert Hendrickson Oregon Health \& Science University, Portland, OR, USA 
Background: Shellfish-borne disease in Alaska is common and results from consumption of dinoflagellate contaminated marine species. Saxitoxin (SXT) and SXT analogue containing shellfish cause paralytic type shellfish poisoning (PSP) with symptoms ranging from GI upset to cardiovascular collapse but is rarely fatal. We present a fatal case of PSP in an adult female from a remote Alaskan village after ingestion of SXT containing shellfish.

Methods: The Oregon Poison Center (OPC) serves Oregon, Alaska and Guam. Medical records were reviewed, as well as mollusk and snail tissue analysis reports from samples sent to the state health department.

Results: A 62-year-old female presented to a remote Alaskan clinic four hours after ingesting harvested shellfish. Initial symptoms were perioral paresthesias and chest pain. Vital signs were significant for tachycardia and hypertension only. Antihistamines and steroids were given for presumed allergic reaction; however, symptoms progressed to frank numbness and extremity weakness. Recommendations were made by OPC for immediate medical evacuation, with early airway management for prolonged transport 1200 miles inland. During transport, the patient sustained six cardiac arrests, and subsequently expired 12 hours after ingestion at the receiving hospital. Stomach contents and uneaten specimens of Blue mussels and Dogwinkle snails in cooking sauce were sent to the Alaska State Laboratory for testing. These were confirmed to be from Dutch Harbor, an area known to be high risk for toxin-containing marine species. Tissue analysis of both the mussels and snails was significant for total SXT and analogue concentrations of $11,200 \mu \mathrm{g} / 100 \mathrm{mg}$ and $287 \mu \mathrm{g} /$ $100 \mathrm{mg}$ respectively (maximum acceptable toxin concentration $<80 \mu \mathrm{g} /$ $100 \mathrm{mg}$ ).

Conclusion: The cause of death in this patient was consistent with severe paralytic shellfish poisoning. Management remains complicated in remote coastal areas, and prevention requires education and surveillance of marine species to prevent both isolated exposures and local outbreaks.

\section{A Severe Case of Ciguatera Poisoning in Guam}

Courtney Temple, Mercedes Wilson, B Zane Horowitz Oregon Health \& Science University, Portland, OR, USA

Background: Ciguatera fish poisoning is a foodborne illness presenting with symptoms ranging from GI upset, to seizures and coma. Severe cases are rare, and most presentations are self-limited. Gambierdiscus toxicus is the primary dinoflagellate producing both ciguatoxin and maitotoxin, in large reef fish who feed on smaller species. We present a severe case of ciguatera poisoning that resulted in near fatal neurologic and hemodynamic compromise.

Methods: The Oregon Poison Center (OPC) serves the state of Oregon, as well as the U.S. territory of Guam. Review of medical records and laboratory testing.

Results: A previously healthy 31-year-old female presented with vomiting, extremity paresthesias, and ataxia thirty minutes after eating a self-prepared stew of reef fish in Guam. An accompanying family member who ate the same meal exhibited similar symptoms. Both were initially discharged. Two days later, she represented with profound weakness, severe paresthesias and confusion. The other family member had improved to baseline. Vital signs and laboratory results were unremarkable. She was intubated for airway protection and admitted to the ICU. There was no evidence of respiratory paralysis; however, she became persistently bradycardic and hypotensive. Her course was complicated by a week of hemodynamic instability requiring high-dose vasopressor and inotropic support, and possible seizures. On day seven, she was extubated and discharged on day nineteen. The fish was identified as the one-spot snapper from a family photo by the FDA. Biologic samples were sent for toxin and metabolite detection. No samples of the meal were available. Results will be presented but are pending at this time.
Conclusion: The clinical course of this patient is consistent with a severe case of ciguatera. As the most commonly reported seafood related illness, identification of cases and confirmatory testing are necessary for clinical care and public education.

\section{The Use of Blue Lotus (Nymphaea Caerulea) in Vaping Devices: a Case Series}

\author{
M D Schimpf ${ }^{1}$, T E Ulmer ${ }^{1}$, H M Hiller $^{2}$, A F Barbuto ${ }^{2}$ \\ ${ }^{1}$ Carl R. Darnall Army Medical Center, Ford Hood, TX, USA. ${ }^{2}$ Carl R. \\ Darnall Army Medical Center, Fort Hood, TX, USA
}

Background: The blue lotus flower (Nypmhaea caerulea) is known for its psychoactive effects, with a history dating back to Ancient Egypt. The psychoactive effects of the flower are attributed to two aporphine alkaloids, apomorphine and nuciferine. Apomorphine is a non-selective dopamine agonist, non-selective alpha antagonist, and a serotonin modulator, with activity as a partial agonist at 5-HT1a and an antagonist at 5HT2a.

Methods: This is a retrospective chart review of four patients who presented to the emergency department with altered mental status following the self-reported use of blue lotus products. The product was delivered either through a vaping device (three of four patients) or as an infused wine (one of four patients).

Results: Four patients were brought via ambulance to the emergency department, where three of four had normal vital signs, and one had tachycardia. No patients had syncope, seizure, or hyperreflexia. One patient had mild hypoxia that resolved with deep inspiration. Significant exam findings included hallucinations and decreased alertness in all patients, with disorientation for one. While one patient's CK was mildly elevated at 3,215 with a creatinine of 1.3 , he did not have symptoms of rhabdomyolysis. No other patient in our series had CK measured. Screening for coingestants with urine immunoassay for drugs of abuse was negative. Testing for apomorphine and nuciferine was not performed. The observation times for these patients until achieving clinical sobriety ranged from two hours and 45 minutes to four hours; all were discharged. Conclusion: Blue lotus is an uncommonly seen hallucinogen that can be used in vaping products. Knowledge of evolving trends in regard to the vaping epidemic can help to provide direction in cases of undifferentiated altered mental status. In this limited series, consumption of blue lotus leads to self-limited symptoms and can be managed supportively.

\section{Desert Rose (Adenium obesum) Ingestion with Quantifiable Digoxin Serum Concentration}

Abdullatif Aloumi, Leena Amine, Yazeed Eldos, Robert J Hoffman Sidra Medicine, Qatar Poison Center, Doha, Qatar

Background: The desert rose (Adenium obesum) is a cardioactive steroid-containing plant. Digoxin-like toxicity after ingestion, measurement of serum digoxin levels, and digoxin Fab therapy to treat toxicity are all described in references. We are unaware of publications documenting quantifiable serum digoxin levels as a marker of exposure to Adenium obesum.

Hypothesis: The null hypothesis is the cardioactive steroids of Adenium obesum will not cross-react with a digoxin assay.

Methods: This is a single-case review of an 8-month-old who ingested Adenium obesum. She had a desert rose flower in her mouth without any indication about quantity ingested. After calling our poison center, her parent was advised to seek pediatric emergency department (ED) evaluation. Upon presentation to the ED, less than one hour later, the patient was asymptomatic with normal vital signs. The patient received one gm/ 
$\mathrm{kg}$ of activated charcoal (AC) after which she vomited. Lab assays including venous blood gas and serum digoxin level were obtained.

Results: The only symptom — vomiting - occurred 15 minutes after administration of $\mathrm{AC}$ was attributed to the $\mathrm{AC}$ use. All lab results were normal with these notable values: serum digoxin level $0.26 \mathrm{nmol} / \mathrm{L}$ $(0.19 \mathrm{ng} / \mathrm{mL})$ and serum potassium $4.2 \mathrm{mmol} / \mathrm{L}$. A 12-lead ECG demonstrated normal sinus rhythm with no apparent digoxin effect. The child was discharged after an uneventful 12-hour post-ingestion observation period. No antidotal digoxin Fab was administered.

Conclusion: This infant ingestion of a desert rose flower with no apparent clinical effects involved a detectable toxin resulting in a measured serum digoxin concentration of $0.26 \mathrm{nmol} / \mathrm{L}(0.19 \mathrm{ng} / \mathrm{mL})$. Cardioactive steroid from Adenium obesum cross-reacted with the assay, an Architect c16000 (Abbott Park, IL, USA). This Adenium toxin exposure resulted in a positive digoxin assay in the absence of clinical findings of toxicity.

\section{Zygacine Toxicity in Three Children}

Lauren T Murphy ${ }^{1,2}$, John A Thompson ${ }^{1,2}$, Stephen T Lee ${ }^{3}$, Annette M Lopez $^{2,4}$, Zane B Horowitz ${ }^{2}$

${ }^{1}$ Oregon Health \& Science University, Portland, OR, USA. ${ }^{2}$ Oregon Poison Center, Portland, OR, USA. ${ }^{3}$ USDA Poisonous Plant Research Laboratory, Agricultural Research Service, Logan, Utah, USA. ${ }^{4}$ Veterans Affairs Portland Healthcare System, Portland, OR, USA

Background: Death camas is a name shared by multiple species within the Melanthiaceae family. Toxicity, including death, is commonly reported in livestock. Human poisoning is rarely reported.

Methods: This is a three-patient case series. Three siblings aged 12, 10, and 7 foraged in their backyard for wild onion for tacos they made for lunch. They collected bulbs from the plants, chopped them, and sauteed them on the stove. Each child ate 1-2 tacos. Approximately 90 minutes later, the 12-year-old developed diarrhea while the two younger children vomited. The mother called the poison center and took the children to the local emergency department. All children had symptomatic bradycardia, were transferred for PICU admission, and displayed repeated episodes of bradycardia responsive to repeated dosing of atropine and intravenous fluids. No child required vasopressors or developed other signs of toxicity. All three children recovered within 24 hours and were discharged within 48 hours. Two smartphone plant identification applications "Picture This" and "PlantNet" erroneously identified the plants as wild onion and wild garlic if pictures did not include the flower in the frame. Plant samples sent to the USDA poisonous plant research laboratory confirmed the presence of zygacine at a concentration up to $3.58 \mathrm{mg} / \mathrm{g}$ in the above ground samples, and up to $1.33 \mathrm{mg} / \mathrm{g}$ in the bulbs.

Results: Death camas contains zygacine (a Veratrum alkaloid), which acts as a sodium and calcium channel opener. Stems and leaves closely resemble wild onion which may result in accidental ingestion. Previous reports of toxicity in humans include bradycardia, hypotension, and gastrointestinal symptoms, with no deaths reported in modern literature. In this series, hypotension appeared to be dependent on the associated bradycardia.

Conclusion: Zygacine causes symptomatic bradycardia responsive to atropine after ingestion of bulbs containing the toxin. Smartphone plant identification applications may misidentify death camas if not all parts are pictured.

137. Prospective Survey of Confidence and Ability to Differentiate Edible and Toxic Mushrooms in the General Population of Thailand

Tuyet Nhi N Tran ${ }^{1}$, Dazhe James Cao ${ }^{1}$, Panee Rittilert ${ }^{2}$, Denis Benjamin $^{3}$, Saphaphume Srisuma ${ }^{2,4}$

${ }^{1}$ UT Southwestern Medical Center, Dallas, TX, USA. ${ }^{2}$ Ramathibodi Poison Center, Bangkok, Thailand, Thailand. ${ }^{3}$ Botanical Research
Institute of Texas, Fort Worth, TX, USA. ${ }^{4}$ Division of Clinical Pharmacology and Toxicology Ramathibodi Hospital, Bangkok, Thailand, Thailand.

Background: Mushroom-related poisonings and deaths occur yearly in Thailand despite warnings from federal agencies.

Research Question: How do the level of confidence, demographics, beliefs, and behaviors characterize the ability to identify toxic and edible mushrooms in the general population of Thailand?

Methods: A prospective survey was distributed via the "Ramathibodi Poison Center" Facebook page from 5/5/2020 to 6/4/2020. Respondents $18+$ years with informed consent were included. Medical toxicologists, pharmacology fellows, and mycologists were excluded. Demographics, confidence, false beliefs, and mushrooms differentiation abilities were collected. Confidence level ranged from 0 to 100 . The median confidence level separated respondents into the low confidence (LC) and high confidence (HC) groups. A 24-picture-quiz assessed mushroom differentiation. $T$-tests, ANOVAs, and linear regressions compared factors with scores. A Shapiro-Wilks test determined data normality.

Results: Of the 340 respondents, $70.9 \%$ were women, $85.9 \%$ never foraged, $5 \%$ foraged $>3$ times, and $61 \%$ had $>1$ false belief. Common beliefs included that edible mushrooms: grew in the same area; do not darken silver; do not change the color of rice/onions once boiled. The median confidence level was 10; thus, 165 respondents were in the HC group and 175 in the LC group. The HC group quiz scores (mean $49.6 \%$, SD $8.8 \%$ ) and the LC group (mean $48.8 \%$, SD $7.5 \%$ ) were not statistically different $(p=\mathrm{NS})$. Those who hiked one-three times/year and foursix/year scored significantly worse than who did not hike $(47.9 \%$ vs. $50.2 \%, p=0.012$, and $43.2 \%$ vs. $50.2 \%, p=0.018$ ). Those who believed edible mushroom grew in the same areas as previous edible mushrooms scored lower ( $47.7 \%$ vs. $49.7 \%, p=0.045)$.

Conclusion: Respondents could not accurately identify mushrooms regardless of confidence with average scores equating to a coin flip. There is a high prevalence of false beliefs that overestimates confidence in consuming potentially life-threatening mushrooms.

138. Intentional Ingestion of Amanita Phalloides Mushrooms with Delayed Presentation and Ethical Considerations in Treatment: a Case Report

Max J Jason, Rachel F Schult, Timothy J Wiegand

University of Rochester Medical Center, Rochester, NY, USA

Background: Few cases discuss management of suicide attempt with amanita phalloides ingestion and describe the natural course of intoxication after delayed presentation. Severe poisoning may require liver transplantation prompting additional ethical considerations after attempted suicide.

Hypothesis: Early involvement of hospital ethics after intentional ingestion of amanita phalloides may help determine goals of care and treatment plans.

Methods: Single-patient chart review. A 72-year-old female presented to the emergency department after one day of nausea, vomiting, and diarrhea. She admitted to intentionally ingesting nine amanita phalloides mushrooms two days prior and requested comfort care. Medical history included suicide attempt two months earlier. Laboratory evaluation identified AST $244 \mathrm{U} / \mathrm{L}$, ALT $221 \mathrm{U} / \mathrm{L}$, and INR 1.2. The patient could not tolerate activated charcoal and nasogastric tube insertion was unsuccessful. Liver enzymes increased and she was placed on IV N-acetylcysteine with a plan to repeat the $100 \mathrm{mg} / \mathrm{kg} / 16$ hours dose until improvement. Ethics was consulted due to refusal of care and rejected refusal of resuscitation but allowed refusal of transplantation with agreement from healthcare proxy.

Results: The patient's clinical status deteriorated. Urine output decreased, and she became encephalopathic prompting intubation three days after presentation. AST, ALT, ammonia, and INR reached peaks of $4548 \mathrm{U} / \mathrm{L}$, 
$8132 \mathrm{U} / \mathrm{L}, 247 \mu \mathrm{mol} / \mathrm{L}$, and undetectably high, respectively. She became hypotensive despite massive volume resuscitation and received vasopressors. The patient extubated to comfort and passed four days after presentation.

Conclusion: Intentional ingestion of amanita phalloides represents an ethical conundrum. Our patient was not allowed to refuse lifesaving medical care despite a pre-existing advanced directive. It was decided that the patient and health care proxy could refuse transplantation which was likely the only option for survival. In cases of intentional ingestion of amanita phalloides, hospital ethics are valuable in establishing goals of care to streamline decision-making across care teams.

\section{Rare Mushroom Toxicity in Upstate New York: Two Cases of Unintentional Lepiota subincarnata Ingestion}

Vincent J Calleo ${ }^{1}$, Tristan W Wang ${ }^{2}$, Jeanna Marraffa ${ }^{1}$, Kathie T Hodge ${ }^{2}$ ${ }^{1}$ SUNY Upstate Medical University, Syracuse, NY, USA. ${ }^{2}$ Cornell University, Ithaca, NY, USA

Background: L. subincarnata is a small mushroom with high amatoxin concentrations and can cause gastrointestinal, renal, pancreatic, and hepatic failure followed by death after ingestion. L. subincarnata ingestion and toxicity is uncommon in the Northeast United States. We present two cases of inadvertent $L$. subincarnata ingestions that develop toxicity.

Methods: A 66-year-old male and 54-year-old female presented to the hospital with vomiting, diarrhea, and abdominal pain. Both consumed foraged mushrooms twelve hours prior to symptom onset. Upon arrival, both patients were hemodynamically stable. They received antiemetics and fluid boluses. N-Acetylcysteine and multi-dose charcoal were started. A mycologist rapidly identified the mushrooms from digital photos as a likely amatoxin-containing Lepiota species. Specimen examination the following day confirmed the identification as L. subincarnata, as did DNA sequencing and phylogenetic analysis. Patients received multidose charcoal for two days and n-acetylcysteine for three days. Both patients had elevations in aspartate transaminase (AST) and alanine transaminase (ALT), with peak ASTs of 200/104 U/L and ALTs of 518/215 $\mathrm{U} / \mathrm{L}$. The female patient developed a creatinine elevation of $1.39 \mathrm{mg} / \mathrm{dL}$ and a lipase elevation of $203 \mathrm{U} / \mathrm{L}$ on day one which both resolved by day three of hospitalization. Both patients improved and were discharged. Results: L. subincarnata is a small mushroom with a relatively high fatality rate whose amatoxin concentration is reportedly higher per gram than Amanita phalloides. Our patients improved with supportive management, multi-dose charcoal, and n-acetylcysteine. Digital photos sent to a mycologist aided in rapid mushroom identification and informed case management.

Conclusion: While infrequently consumed, providers should be aware L. subincarnata is present in the Northeast United States. Digital photos coupled with mycologist collaboration facilitate mushroom identification.

\section{Fatal Endothall Herbicide Ingestion}

Dimity CA McCracken ${ }^{1}$, Richard McNaulty ${ }^{2,3}$, Christopher T Dugan ${ }^{4}$, Andrew H Dawson ${ }^{5,6}$, Nicholas A Buckley ${ }^{5,6}$

${ }^{1}$ Orange Health Service, Orange, NSW, Australia. ${ }^{2}$ Blacktown Mount Druitt Hospital, Sydney, NSW, Australia. ${ }^{3}$ Western Sydney Toxicology Service, Sydney, NSW, Australia. ${ }^{4}$ Liverpool Hospital, Sydney, NSW, Australia. ${ }^{5}$ The Children's Hospital Westmead, Sydney, NSW, Australia. ${ }^{6}$ The University of Sydney, Sydney, NSW, Australia

Background: Endothall is a widely available selective serine-threonine protein phosphatase (PP2A) inhibiting herbicide and plant desiccant. We are aware of only two case reports of deliberate self-poisoning in the literature, both fatal (Allender, 1983 and Day, 1988).

Hypothesis: Endothall is highly lethal in deliberate self-poisoning.
Methods: This is a single medical chart review.

Results: A young adult patient was brought to hospital after admitting to a deliberate self-poisoning by ingestion of $50 \mathrm{~mL}$ of $175 \mathrm{~g} / \mathrm{L}$ endothall dipotassium salt 2.5 hours previously with no co-ingestants. Family members corroborated the history and an empty bottle was presented to staff. Initially, the patient was alert and had hematemesis, abdominal pain, tachycardia, BP $100 / 70 \mathrm{mmHg}$, and metabolic acidosis with lactate of $4.8 \mathrm{mmol} / \mathrm{L}$. A pantoprazole infusion and supportive care was commenced. Toxicology services advised there was no specific treatment but multi-organ failure was likely, noting previous fatalities. Thereafter, the patient deteriorated over the next few hours and became hypotensive and obtunded. Metabolic acidosis worsened with a pH 6.9 and bicarbonate $12 \mathrm{mmol} / \mathrm{L}$, accompanied by low serum glucose, potassium, magnesium, and phosphate. Urine output dropped to almost zero and there was an acute kidney injury with a creatinine of $121 \mathrm{mmol} / \mathrm{L}$. Despite aggressive therapies which included dialysis, noradrenaline, vasopressin, intralipid, mechanical ventilation and blood products, the patient developed acute respiratory distress syndrome, disseminated intravascular coagulopathy, and spontaneous intra-abdominal bleeding. Unfortunately, the patient arrested and died 13 hours post-ingestion.

Conclusion: There is mounting evidence that deliberate endothall ingestion is highly lethal and is characterised by gastrointestinal effects followed by multi-organ dysfunction syndrome. Consideration should be given to regulating its availability.

141. Acute Renal Failure from Ingestion of Wood Bleach Intended for Use as a Miticide in Beekeeping

Christopher N Sweat ${ }^{1,2}$, Carrie L Oakland ${ }^{1}$, Rachel D Brandt ${ }^{1}$, Jon B Cole $^{1,3}$, Travis D Olives ${ }^{1,3}$

${ }^{1}$ Minnesota Poison Control System, Minneapolis, MN, USA. ${ }^{2}$ Regions Hospital, Saint Paul, MN, USA. ${ }^{3}$ Hennepin Healthcare, Minneapolis, $M N$, USA

Background: Used as a lightening agent for lumber and to remove tannin stains, wood bleach is a $>95 \%$ pure formulation of oxalic acid that is clearly labeled as a poison. While not marketed as a pesticide, it is used off-label by hobbyist beekeepers to treat infestations of Varroa mites (Varroa destructor).

Hypothesis: Ingestion of wood bleach can result in nephropathy similar to ethylene glycol poisoning.

Methods: This is a single-patient case report. A 46-year-old man presents to the ED for nausea and vomiting after accidental ingestion of a mouthful of a 1:4 mixture of what was reported as "bleach and grain ethanol intended for use in beekeeping."

Results: Initial evaluation was notable only for a creatinine of 2.46. The patient was otherwise well appearing, so he was volume resuscitated and discharged. Five days later, he returned endorsing ongoing intentional use of the mixture as an inebriant, with worsening nausea and vomiting. He was alert, fully oriented and in no distress with normal vitals save hypertension to $175 / 110 \mathrm{mmHg}$. Laboratory evaluation revealed worsened acute renal failure (creatinine $16.8 \mathrm{mg} / \mathrm{dL}$ ) and metabolic acidosis $(\mathrm{pH}$ 7.3 , bicarbonate $15 \mathrm{mmol} / \mathrm{L}$, anion gap 29). The patient was given fomepizole, supplementary thiamine and vitamin B6 for suspected occult ethylene glycol poisoning, and hemodialysis was initiated. On further discussion, it was learned that the mixture contained 'wood bleach' ( $>$ $95 \%$ oxalic acid) rather than the reported sodium hypochlorite. Renal biopsy on hospital day six confirmed oxalic nephropathy. He was discharged hospital day eight with a creatinine of $5.7 \mathrm{mg} / \mathrm{dL}$ and plans for ongoing hemodialysis.

Conclusion: 'Wood bleach' contains oxalic acid that may induce oxalate nephropathy similar to ethylene glycol if ingested. It should be considered in the differential of patients with acute renal failure who participate in beekeeping. 
142. Intentional Bromethalin Ingestion Resulting in Lactic Acidosis, Severe Delayed CNS Depression

Bryan J Ross, Blake A Froberg, Daren M Beam

Indiana University School of Medicine, Indianapolis, Indiana, USA

Background: Bromethalin is a neurotoxic rodenticide that functions by disrupting mitochondrial oxidative phosphorylation; its use has increased since 2008 following restrictions on anticoagulant rodenticides.

Methods: An 87-year-old male ingested a slurry of 56 grams of $0.01 \%$ bromethalin $(0.06 \mathrm{mg} / \mathrm{kg})$ Tomcat Mouse Killer ${ }^{\circledR}, 300 \mathrm{mg}$ trazodone and boric acid. Gastric lavage yielded green frothy material. Fifty grams of activated charcoal was administered; magnesium and potassium were given for a QTc $527 \mathrm{msec}$. Serial serum lactates obtained, peaking at $5.6 \mathrm{mmol} / \mathrm{dL} 9$ hours PI, down to $3.5 \mathrm{mmol} / \mathrm{dL} 26$ hours later. The patient became obtunded 16 hours PI; subsequent CT and MRI were normal. His mentation fluctuated over the next four days; he expired following a likely aspiration event secondary to CNS depression.

Results: Lethality of bromethalin after acute ingestion is poorly described in the literature. One case of bromethalin ingestion of $0.33 \mathrm{mg} / \mathrm{kg}$ was described, causing lethal cerebral edema with delayed onset of toxicity. Our case similarly displayed delayed neurological symptoms developing approximately 16 hours post-ingestion, but at a much smaller reported dose $(0.06 \mathrm{mg} / \mathrm{kg})$. The gradual rise of serum lactate levels may be indicative of inhibition of aerobic respiration, although peak lactate level of 5.6 $\mathrm{mmol} / \mathrm{dl} \mathrm{did} \mathrm{not} \mathrm{seem} \mathrm{to} \mathrm{correlate} \mathrm{with} \mathrm{onset} \mathrm{of} \mathrm{neurological} \mathrm{symptoms.}$ The exact cause of his neurological decompensation is unclear; CT and MRI showed no evidence of cerebral edema described in previous cases. The clinical picture is clouded by co-ingestion of boric acid and trazodone; neither ingestion explains the patient's delayed neurological decline or time frame of symptoms.

Conclusion: Bromethalin is potentially toxic at ingestions below the previously described lethal dose. In this case, severe neurological symptoms were observed to develop hours to days after initial exposure.

\section{Assessment of Serum Immunoglobulin $\mathbf{E}$ Changes in Chronic Alcohol Consumption}

Kyaw Soe Htun, Myint Myint Naing, Ko Ko Win, Zaw Min Tun, Kyaw Soe, Thein Zaw, Kyee Myint

Defence Services Medical Research Centre, Tatkone, Naypyitaw, Myanmar

Background: Alcohol consumption, a pervasive social problem, has been a worldwide epidemic for centuries. According to the $2014 \mathrm{WHO}$ data, the prevalence of alcohol use disorders in males was $2.7 \%$ and that of females was $0.5 \%$. Alcoholic liver diseases may alter immune regulation results in secondary immunodeficiency and autoimmunity. Immunoglobulins are raised once the liver damage has begun, and their values may be directly proportional to the degree of alcohol induced liver damage. This study was aimed to explore serum IgE status in chronic alcohol consumption.

Methods: This laboratory based descriptive study conducted on 192 participants and drinking status of all participants was assessed by Seung-Hyun Ma's study. Blood samples were collected and then serum IgE was detected by usinga Maglumi 800 Immunology Analyzer.

Results: The value above $190 \mathrm{IU} / \mathrm{ml}$ was remarked as increasing serum IgE level. The mean age of participants was $43.4 \pm 7.2$ and the overall prevalence of smoking and betel chewing in all participants were $56.2 \%$ and $52.1 \%$ respectively. Increasing the years of drinking duration that was significantly associated with appearance of alcohol withdrawal syndrome $(p=0.001)$ and dyspnoea of exertion $(p=0.001)$. Serum IgE level was increasing in 91 (48.4\%) of all participants. Apart from tobacco habits, increasing serum IgE level was strongly associated with age and drinking status.
Conclusion: This study highlighted the importance of rapid and early recognition of $\operatorname{IgE}$ changes in chronic alcoholic patients which are transient and can be reversed by abstinence of alcohol using.

\section{Para-dichlorobenzene Pica: a Fatal Case of PDCB-Related} Leukoencephalopathy

Brian R. Bush ${ }^{1}$, Trevonne M. Thompson ${ }^{2}$

${ }^{1}$ Toxikon Consortium, Chicago, IL, USA. ${ }^{2}$ University of Illinois College of Medicine - Chicago, Chicago, IL, USA

Background: Para-dichlorobenzene (PDCB) is a chlorinated hydrocarbon used as an air, toilet, and urinal deodorizer. When misused chronically, neurotoxicity can occur. We report a case of chronic ingestion of PDCB toilet deodorizer resulting in muscle rigidity, catatonia, and progressive leukoencephalopathy.

Methods: A 33-year-old female presented as a consult to our medical toxicology service for possible toxic ingestion resulting in prolonged catatonia. Nine days before consultation, the patient presented to an outside hospital (OSH) emergency department from a psychiatric facility for acute unresponsiveness over the preceding 12 hours. In the OSH ED, she was unresponsive with rigid extremities requiring intubation. She was admitted to the ICU. After a negative evaluation at the OSH, she was transferred to our hospital's ICU for electroconvulsive therapy for presumed malignant catatonia. She did not respond to treatment; our service was then consulted. A brain MRI demonstrated toxic leukoencephalopathy. Collateral information provided by the patient's family noted a seven-month history of ingesting PDCB-containing toilet deodorizing cakes. Review of our poison center records revealed that this patient presented to a different hospital with weakness and "odd behavior" six weeks prior to this admission but left against medical advice. After an extensive evaluation, an alternative diagnosis was not identified. The leukoencephalopathy worsened on repeat MRI. On hospital day 24, the patient's family withdraw care; she expired the following day.

Results: PDCB ingestion and inhalation have been reported to cause toxic leukoencephalopathy. PDCB is lipophilic and accumulates in adipose with chronic exposure and can demyelinate neurons. Patients can be misdiagnosed with a primary psychiatric disorder given PDCB's indolent course and progressive decline. Thorough history and collateral information are essential in the identification of such cases.

Conclusion: We report a case of progressive toxic leukoencephalopathy and death in the context of chronic para-dichlorobenzene abuse.

\section{Pediatric Hydrocarbon Exposure with Delayed Respiratory Symptoms}

Wells Brambl ${ }^{1}$, Stephanie L Widmer ${ }^{2}$, Josh N Nogar ${ }^{1}$

${ }^{I}$ Northwell Health, Queens, NY, USA. ${ }^{2}$ Nort, Queens, NY, USA

Background: After a hydrocarbon ingestion, the standard of care among most toxicologist is to monitor for 6 hours for the development of respiratory symptoms, and discharge if asymptomatic. Here we present the case of a patient who developed clinically significant symptoms 24 hours after exposure to benzene.

Methods: The patient is a 4-year-old male with no significant past medical history who presented to the emergency department after a reported exploratory benzene ingestion. The grandmother had brought home a benzene-containing cleaning product from her work in a water bottle. The child ingested a mouth full and was brought to the ED for evaluation approximately one hour after ingestion. The child initially had normal vital signs and a normal physical exam. He was monitored for a period of six hours and discharged. Approximately 24 hours later, he was noted to have a fever of $102 \mathrm{~F}$, but otherwise had normal vital signs for his age. Upon return to the ED, he had abdominal pain and pleurisy. A chest 
radiograph demonstrated bilateral opacities. He was given an empiric dose of antibiotics in the ED and admitted for monitoring. Antibiotics were discontinued and he remained afebrile. The patient had an unremarkable course and was discharged. A urinary phenol level assay was not able to be performed due to discontinuation of the assay by Quest Diagnostics.

Conclusion: A clinically relevant delayed pneumonitis may occur more than six hours after exposure to benzene. This observation period may not be sensitive enough to capture all potentially ill patients.

\section{6. "Maximum Impact": Cardiac Arrest Following Ethyl Chloride Exposure}

Elizabeth T Jacobs ${ }^{1,2}$, Andrew M King ${ }^{1,2}$, Cynthia K Aaron ${ }^{1}$, Varun Vohra $^{1}$

${ }^{1}$ Michigan Poison Center, Wayne State University School of Medicine, Detroit, MI, USA. ${ }^{2}$ Detroit Medical Center, Detroit, MI, USA

Background: "Poppers", historically alkyl nitrites, are used predominantly as an aphrodisiac by men who have sex with men, due to their vasodilating properties. These substances are low cost and easily accessible alternatives to prescription drug options. "Maximum Impact", sold as a cleaning solvent, is disguised as a "popper" but instead contains the halogenated hydrocarbon, ethyl chloride, putting users at risk for cardiac dysrhythmias and cardiac arrest via myocardial sensitization. Both reversible neurotoxicity and death secondary to ethyl chloride have been described in the literature.

Hypothesis: Misuse of ethyl chloride may lead to cardiac arrest.

Methods: This is a report of a 28 -year-old male who experienced cardiac arrest while inhaling (huffing) ethyl chloride.

Results: A 28-year-old male with history of daily solvent abuse was found in cardiac arrest after huffing "Maximum Impact". Upon arrival of Emergency Medical Services he had pulseless electrical activity. After continued prehospital resuscitative efforts, there was return of spontaneous circulation. On arrival to the emergency department (ED), his ECG demonstrated HR 115 bpm, QRS 114 msec "with significant terminal R wave deflection", and QTc $466 \mathrm{msec}$. He was started on norepinephrine and vasopressin for hypotension. Acetaminophen, salicylates, and ethanol were undetectable; laboratories were significant for lactate of 12 $\mathrm{mMol} / \mathrm{L}$. Initial $\mathrm{CT}$ head was concerning for cerebral edema. Hypothermic protocol was initiated in the ED prior to ICU admission. During his ICU course, he had no brainstem reflexes, developed coagulopathy, and multiorgan failure. Family withdrew care and he died on day two of hospitalization.

Conclusion: Misuse of ethyl chloride can lead to cardiac arrest. Ethyl chloride may be confused with alkyl nitrites ("poppers") and lead to significant toxicity, including death.

\section{Cluster of Occupational Nitric Acid Exposures with Spectrum of Clinical Severity: ECMO to Outpatient Management}

Alexander M Sidlak ${ }^{1}$, Amanda S Korenoski ${ }^{2,3}$, Michael J Lynch ${ }^{4}$ ${ }^{1}$ Inova Fairfax, Falls Church, VA, USA. ${ }^{2}$ University of Pittsburgh School of Pharmacy, Pittsburgh, PA, USA. ${ }^{3}$ Pittsburgh Poison Center, Pittsburgh, PA, USA. ${ }^{4}$ University of Pittsburgh School of Medicine, Pittsburgh, PA, USA

Background: Nitric acid combines with oxygen when heated to produce nitric oxides, which can lead to acute respiratory distress syndrome (ARDS). Toxicity is typically delayed given nitric oxides' low solubility.

Methods: Seven workers at a metal processing company were exposed to nitric acid, which was used to polish stainless steel sheets at the company. Liquid nitric acid was left being heated and a gaseous orange cloud was noted to be emanating from the area. Three patients required hospitalization. A 49-year-old man close to the exposure developed immediate respiratory distress. He was intubated on presentation to a local hospital. The next day, he developed ARDS and was transferred to a tertiary center where he was started on extracorporeal membrane oxygenation (ECMO). $\mathrm{He}$ additionally required vasopressors for shock, antibiotics, and stress dose steroids. On day five of hospitalization, he had improved ventilator settings and was decannulated on day 10 with continued improvement after. Two other workers required hospitalization for hypoxemia with respiratory symptoms developing the day after exposure. The remaining four patients had minor symptoms in the emergency department, were discharged, and subsequently lost to follow-up.

Results: Nitric oxides produce cellular injury in alveoli leading to respiratory injury that can progress to ARDS depending on the concentration and duration of exposure. Apart from standard supportive care, there are no antidotal agents. Prior case reports have described significant injury with death despite ECMO. Early initiation and transfer for ECMO was initiated for the most severe case in this patient cohort.

Conclusion: This case series showcases the range in clinical symptoms after heated nitric acid exposure. ECMO led to a successful recovery in the patient with the most significant toxicity.

\section{Extreme Lactate Elevation in Firefighters Without Cyanide Toxicity}

Fiona Garlich ${ }^{1,2}$, Meghan B Spyres ${ }^{3,4}$

${ }^{1}$ Keck School of Medicine, University of Southern California, Los Angeles, CA, USA. ${ }^{2}$ LAC+USC Medical Center, Los Angeles, CA, USA. ${ }^{3}$ University of Arizona College of Medicine, Phoenix, AZ, USA. ${ }^{4}$ Banner University Medical Center, Phoenix, AZ, USA

Background: In fire victims with smoke inhalation, severe metabolic acidosis with lactate $>10 \mathrm{mmol} / \mathrm{L}$ is typically attributed to cyanide. Other causes of lactate elevation in this setting are underrecognized.

Hypothesis: Extremely high lactates in fire victims may be secondary to non-cyanide etiologies.

Methods: This is a retrospective case series. Following a massive explosion at a cannabis product storage facility, 12 firefighters were transported to the Emergency Department with thermal injuries. All had struggled to escape the burning structure while wearing heavy protective gear. Serial vital signs and laboratory analysis was performed.

Results: On presentation, three firefighters had metabolic acidosis with extreme lactate elevation. Patient 1: lactate $26.6 \mathrm{mmol} / \mathrm{L}, \mathrm{pH}$ 6.99 , bicarbonate $5 \mathrm{mmol} / \mathrm{L}$; patient 2: lactate $21.8 \mathrm{mmol} / \mathrm{L}$, $\mathrm{pH} 7.22$, bicarbonate $9 \mathrm{mmol} / \mathrm{L}$; patient 3 : lactate $>20 \mathrm{mmol} / \mathrm{L}$ (venous blood gas level), pH 7.36, bicarbonate $8 \mathrm{mmol} / \mathrm{L}$. Carboxyhemoglobin levels were all $<1 \%$. All patients had tachycardia (HR 96-130 bpm) without hypotension (BP 113-153/64-80 mm Hg), and were alert with normal mental status. After fluid resuscitation and $100 \%$ oxygen, labs were repeated at approximately 30 minutes. Patient 1 : lactate $13.7 \mathrm{mmol} / \mathrm{L}, \mathrm{pH} 7.25$, bicarbonate $12 \mathrm{mmol} / \mathrm{L}$; patient 2 : lactate $5.8 \mathrm{mmol} / \mathrm{L}, \mathrm{pH} 7.34$, bicarbonate $22 \mathrm{mmol} / \mathrm{L}$; patient 3: lactate $7.9 \mathrm{mmol} / \mathrm{L}, \mathrm{pH} 7.58$, bicarbonate $14 \mathrm{mmol} / \mathrm{L}$. Treatment with hydroxocobalamin was withheld due to hemodynamic stability, normal mental status, and improving acid-base status. Lactates continued to decline. Serum cyanide levels at 90 minutes returned undetectable in all three patients. No other cause of metabolic acidosis could be found other than extreme exertion.

Conclusion: Maintaining a high suspicion of cyanide toxicity in fire victims with elevated lactate is imperative. Alternate causes, including extreme exertion, should be considered in alert, normotensive patients with rapidly-declining lactate. 
149. Dermal Toxicity from Exposure to Urine from a Paraquat Poisoned Patient

Ana Castaneda-Guarderas ${ }^{1}$, Taylor Chesser $^{2}$, Mark F Brady ${ }^{3}$, Rebecca E Bruccoleri $^{4}$

${ }^{1}$ Department of Emergency Medicine, University of Tennessee Health Science Center, Memphis, TN, USA. ${ }^{2}$ Regional One Health, Memphis, TN, USA. ${ }^{3}$ Department of Emergency Medicine, Alpert Medical School of Brown University, Providence, RI, USA. ${ }^{4}$ Tennessee Poison Center, Nashville, TN, USA

Background: While paraquat is known to be absorbed through the skin and eliminated by the kidneys, the effect of urine from someone who ingested paraquat on the skin is not well described. Paraquat is eliminated unchanged in the urine since it does not undergo metabolism.

Hypothesis: Skin exposure to urine from paraquat poisoned patients can result in dermal toxicity.

Methods: This is a single-patient case report. A 25-year-old female nurse placed a Foley catheter in a patient who accidentally ingested 1.8 ounces of paraquat while the patient was intubated. Despite using standard personal protective equipment for Foley catheter placement, including sterile latex gloves, some of the patient's urine got onto the nurse's right forearm. The nurse immediately flushed it with water and washed with soap and water.

Results: The nurse developed blistering covering $1 \%$ body surface area consistent with a partial thickness chemical burn within 30 minutes of contact. She was evaluated by the burn surgery service and discharged after observation in the burn center. Besides pain, the nurse had some nausea which resolved after one dose of ondansetron in the burn center. She had no other systemic symptoms. The blistered skin painfully sloughed off within $24 \mathrm{hrs}$.

Conclusion: Based on EPA references and also what is available in an Emergency Department, we suggest extended personal protective equipment recommendations for staff caring for patients who have ingested paraquat should include long sleeves, chemical resistant gloves (i.e., nitrile rubber $\geq 14$ mils), plastic gown, and face shield/eye protection. This recommendation should apply even after external decontamination since the urine itself can be a significant dermal toxin.

150. Longitudinal Neurological Deterioration and MRI Changes in a Case of Paradichlorobenzene Poisoning

Daniel Nogee ${ }^{1,2}$, Matthew Eisenstat ${ }^{1,2}$, Kyle Suen ${ }^{1,2}$, Khalid Alaufi ${ }^{1,2}$, Emily Kiernan $^{1,2}$, Ziad Kazzi ${ }^{1,2}$

${ }^{1}$ Emory University, Atlanta, GA, USA. ${ }^{2}$ Georgia Poison Center, Atlanta, GA, USA

Background: Paradichlorobenzene (PDCB) is a chlorinated aromatic hydrocarbon used in industrial and household applications, including toilet bowl cleaners/deodorizers. Intentional inhalation or ingestion of PDCB can result in severe toxic leukoencephalopathy.

Methods: A 34-year-old woman presented to the Emergency Department (ED) with progressively worsening ambulatory dysfunction, memory loss, and hallucinations over several weeks; family reported that the patient intentionally inhaled toilet bowl cleaner/ deodorizer crystals containing PDCB. Urine testing for the metabolite 2,5-dichloropheneol (DCP) was performed and magnetic resonance imaging (MRI) of the brain obtained. The patient was discharged home on hospital-day three. Six months later, she presented again to the ED with worsening cognitive deficits, confusion, difficulty walking, fatigue, and weight loss, in the context of continued recreational PDCB inhalation. She was admitted and brain MRI, urine DCP, and blood PDCB levels were obtained. A PEG tube was placed due to the patient's poor nutritional status, and after a fiveweek admission she was discharged to a skilled nursing facility.
Results: Initial urine DCP level was $260 \mathrm{mg} / \mathrm{L}$. Six months later, urine DCP level was $770 \mathrm{mg} / \mathrm{L}$ and blood PDCB level was $7.4 \mathrm{mcg} / \mathrm{mL}$. Initial brain MRI showed no abnormalities; repeat MRI six months later showed widespread abnormal increased T2/FLAIR signal within bilateral cerebral white matter, internal capsules, corpus callosum, midbrain, and mammillary bodies. A Montreal Cognitive Assessment test was completed on the second presentation and resulted in a score of five out of 30. Specific deficits were noted in visuospatial/executive, attention, abstraction, and delayed recall areas.

Conclusion: PDCB toxicity from misuse of household cleaning products can result in significant neuro-cognitive deficits. This patient's multiple admissions allowed for a longitudinal examination of the clinical, neuroimaging, and laboratory progression of PDCB toxicity.

\section{Succimer Mitigates Peri-operative Risk for Increase in Blood} Lead Levels During Bulletectomy

Joseph M Kennedy ${ }^{1,2}$, Joanne C Routsolias ${ }^{1,2}$, Mark B Mycyk ${ }^{1,2}$

${ }^{I}$ Cook County Health, Chicago, IL, USA. ${ }^{2}$ Toxikon Consortium, Chicago, IL, USA

Background: Manipulation of retained bullet fragments (RBFs) during bulletectomy can result in clinically significant elevations in blood lead levels (BLL).

Hypothesis: Chelation with succimer perioperatively can mitigate risk for an increase in BLLs that accompany intraoperative RBF manipulation.

Methods: This is a single-patient retrospective chart review. A 38year-old male (weight $84 \mathrm{~kg}$ ) sustained a gunshot wound to his left knee 20 years prior to his initial toxicologic evaluation. He was first evaluated in the ED for knee pain after dancing and found to have several large RBFs with diffuse synovial calcifications and a BLL of $69 \mathrm{mcg} / \mathrm{dL}$. He was lost to follow-up and returned one year later in police custody with a comminuted tibial plateau fracture requiring operative repair. Because of his previous elevated BLL and the RBFs in his knee, chelation was requested to prevent an iatrogenic increase in his BLL during his operation.

Results: Peri-operative chelation treatment with succimer was done because of a national shortage of $\mathrm{CaNa}_{2}$ EDTA. His BLL three days following the fracture was $58 \mathrm{mcg} / \mathrm{dL}$. An initial five-day load of succimer ( $800 \mathrm{mg}$ TID) reduced the BLL to $19 \mathrm{mcg} / \mathrm{dL}$ at 0900 on the day of the operation; BLL at 1900 on the evening of the operation was $15 \mathrm{mcg} / \mathrm{dL}$. Succimer was continued for a total of 19 days. Ten days after completing chelation, the BLL was $21 \mathrm{mcg} / \mathrm{dL}$. The patient had no adverse events and had an uneventful convalescent period.

Conclusion: Previous reports suggest operative repair involving intraarticular RBFs can increase BLL significantly. Drug shortages continue to challenge optimal treatment strategies. This case included the expertise of a multi-disciplinary team of surgeons, medical toxicologists, pharmacists, and jail medicine specialists. Oral succimer monotherapy here was an inexpensive, safe, and reasonable alternative to intravenous chelation in this patient undergoing bulletectomy.

\section{Intentional Overdose of Mercuric Chloride with Delayed Chelation}

Courtney Temple, Beth Chandler, B Zane Horowitz

Oregon Health \& Science University, Portland, OR, USA

Background: Inorganic mercury salts, used historically as disinfectants, are no longer utilized clinically given severe toxicity and organ dysfunction, particularly renal failure. These salts, such as mercuric chloride $\left(\mathrm{HgCl}_{2}\right)$, are currently available as stabilizing agents and chemical reagents for laboratory research. 
Methods: Medical records were reviewed. Concentrations were determined from a reference laboratory.

Results: A 31-year-old male ingested mercuric chloride in a self-harm attempt by mixing a "spoonful of powder" into a glass of water. On EMS arrival, he was vomiting and complaining of severe abdominal pain. Empiric chelation and aggressive hydration was recommended for symptomatic ingestion of nearly 15 grams of $\mathrm{HgCl}_{2}$, with confirmatory testing to result in six days. Initial labs were significant for a normal BUN and creatinine, and vital signs for tachycardia and hypertension. BAL was reportedly on back order at almost all surrounding hospitals, but three doses were ultimately discovered at one hospital. Shortly after transfer, the patient exhibited hematemesis but remained hemodynamically stable. Chelation with BAL was started seven hours after ingestion. Refractory vomiting precluded early succimer administration. He received the three available doses of BAL, and was transitioned to oral succimer. Whole blood mercury concentration was $2747.62 \mathrm{mcg} / \mathrm{L}$ (nl 0-10 mcg/L), with 24-hour urine concentration $>3000 \mathrm{mcg} / \mathrm{L}$ (unable to further dilute). After a 19-day course of chelation, whole blood concentration resulted at 53.8 $\mathrm{mcg} / \mathrm{L}$, and urine concentration $137-193 \mathrm{mcg} / \mathrm{L}$. After 30 days, he was placed in a psychiatric facility without signs of organ failure or hemodynamic instability despite initial high concentrations of inorganic mercury identified in blood and urine.

Conclusion: Despite a significant ingestion of mercuric chloride and delayed chelation, this patient did not develop renal failure or neurologic sequelae from the exposure.

\section{Chronologically Distant Mercury Toxicity Cases from Single Spill Source}

John A Thompson ${ }^{1,2}$, Robert G Hendrickson ${ }^{1,2}$, Adrienne Hughes ${ }^{1,2}$, Zane Horowitz ${ }^{1,2}$

${ }^{1}$ Oregon Health and Science University, Portland, OR, USA. ${ }^{2}$ Oregon Poison Center, Portland, OR, USA

Background: Elemental mercury can vaporize at room temperature creating a potential for inhalational toxicity. Spill cleanup is difficult and can potentially further vaporize mercury.

Methods: This is a single incident. The poison center was called about a patient with mercury toxicity approximately 62 days since his exposure. Two weeks later, another patient with mercury toxicity was reported. It was determined that these cases were linked to the same mercury spill. Patient one: serum mercury $>160 \mu \mathrm{g} / \mathrm{L}$. Presented with shortness of breath, hypoxia, and mouth pain. Chelated with British Anti-Lewisite and dimercaptosuccinic acid. Patient two: serum mercury $>160 \mu \mathrm{g} / \mathrm{L}$. Presented with nausea, abdominal pain, and arthralgias. Chelated with dimercaptosuccinic acid. The Environmental Protection Agency (EPA) and local authorities were contacted. The mercury air concentration in the doorway was greater than the limit of detection on the EPA's sensor $\left(>500,000 \mathrm{ng} / \mathrm{m}^{3}\right)$. Beads of liquid mercury were found underneath the floorboards where the spill occurred after the first patient tried to extract gold from computer parts. The first patient attempted cleaning with a vacuum. The second patient was not involved in cleaning.

Results: Elemental mercury is a heavy metal that can be obtained from multiple sources. It is sometimes used to separate gold from impure sources. This process requires vaporizing mercury, which can lead to significant mercury exposure. If done indoors, porous materials such as clothing, bedding, and the structure itself can absorb mercury then release the vapors based on environmental factors, prolonging the exposure period.

Conclusion: We report two mercury exposures from a single spill separated over about two months. These cases highlight the danger of mercury vapors and the importance of proper techniques regarding cleanup, disposal, and decontamination following a spill.
154. Toxin Induced Thrombocytopenia and Other Hematologic Abnormalities in Mercury Toxicity

John A Thompson ${ }^{1,2}$, Robert G Hendrickson ${ }^{1,2}$

${ }^{I}$ Oregon Health and Science University, Portland, OR, USA. ${ }^{2}$ Oregon Poison Center, Portland, OR, USA

Background: We present a case of a delayed diagnosis of mercury toxicity with substance induced thrombocytopenia.

Methods: This is a single-case report. A 42-year-old male presented with shortness of breath, mouth pain, and epistaxis. He was hypoxic on arrival. Chest $\mathrm{x}$-ray showed findings suggestive of atypical pneumonia. His blood chemistry showed a thrombocytopenia (PLT $\left.0 \times 10^{9} / \mathrm{L}\right)$, leukocytosis $(16$ $\times 10^{9} / \mathrm{L}$ ), lymphopenia $(6.1 \%)$, eosinophilia $(32.0 \%)$, and anemia (hemoglobin $11.9 \mathrm{~g} / \mathrm{dL}$ ). His thrombocytopenia did not respond to platelet transfusion. His course was complicated by an intracranial hemorrhage. Hematology formed a diagnosis of immune thrombocytopenic purpura and recommended treatment for this and presumed eosinophilic pneumonia with IVIG and steroids. An extensive infectious workup was unrevealing. His thrombocytopenia was recurrent during his hospitalization. Weeks after his presentation, a mercury test was obtained and his serum concentration was greater than the limit of detection of $160 \mu \mathrm{g} / \mathrm{L}$. The poison center was consulted. Physical exam was notable for intention tremor and an irritating sensation on the hands with lichenification. $\mathrm{He}$ received chelation with British Anti-Lewisite and subsequently dimercaptosuccinic acid with improvement in symptoms he was discharged to a skilled nursing facility.

Results: Mercury toxicity is reported to cause many hematologic effects. Physical exam findings are varied and without history make diagnosis difficult. Our patient's presentation was concerning for inhalation of mercury vapors; however, diagnosis was delayed as there was no history provided. He had exam findings suggestive of acrodynia, which improved with chelation. No other causes for his thrombocytopenia were found after an extensive hematologic and infectious workup, suggestive of toxin-induced thrombocytopenia.

Conclusion: We report a case of mercury toxicity with toxin-induced thrombocytopenia and intracranial hemorrhage. Providers should be aware of the hematologic problems associated with mercury toxicity.

\section{Case Report: Successful Lead Chelation with DMSA During Pregnancy}

Joshua Nogar, Stephanie Widmer, Wells Brambl

Northwell Health, Manhasset, NY, USA

Background: Very little data exist to guide lead chelation treatment strategies in pregnancy. We present a case of successful lead chelation with DMSA in a pregnant patient with a peak lead level of $55 \mu \mathrm{g} / \mathrm{dL} / \mathrm{dL}$. Methods: A 38-year-old female presented to her OB/GYN at 8 wks gestational age (EGA), after a screening capillary lead level resulted at 50 $\mathrm{mcg} / \mathrm{dL}$; the confirmatory serum lead level was $47 \mathrm{mcg} / \mathrm{dL}$; RBC porphyrin $671 \mu \mathrm{g} / \mathrm{dL} / \mathrm{dL}($ ref $<80 \mathrm{mcg} / \mathrm{dL}$ ); hemoglobin $8.5 \mathrm{~g} / \mathrm{dL}$. The source of her lead exposure was an Ayurvedic medication consumed prior to conception. After removal from the source, her levels were trended into the second trimester: $47 \mu \mathrm{g} / \mathrm{dL}$ (9wks EGA), $44 \mu \mathrm{g} / \mathrm{dL} / \mathrm{dL}$ (11wks EGA), $42 \mu \mathrm{g} / \mathrm{dL} / \mathrm{dL}$ (14 weeks EGA), $55 \mu \mathrm{g} / \mathrm{dL} / \mathrm{dL}$ (15 week EGA). Chelation was initiated at 16 weeks EGA with a 19-day course of DMSA $(350 \mathrm{mg}$ / $\mathrm{m} 2$ tid $\mathrm{x} 5 \mathrm{~d}$, then $350 \mathrm{mg} / \mathrm{m} 2$ bid $\times 14 \mathrm{~d}$ ). At the conclusion of chelation, her lead level was $16 \mu \mathrm{g} / \mathrm{dL} / \mathrm{dL} ;$ RBC porphyrin $552 \mu \mathrm{g} / \mathrm{dL} / \mathrm{dL}$; hemoglobin $11.6 \mathrm{~g} / \mathrm{dL}$. Her liver function remained normal throughout chelation. The lead level two weeks after chelation had rebounded to $23 \mu \mathrm{g} / \mathrm{dL} /$ $\mathrm{dL}$, and another 19-day course of DMSA was prescribed. She is presently 20 weeks EGA, with an estimated due date of 3/29/21.

Conclusion: Outpatient DMSA chelation appears to be well-tolerated and effective at lowering maternal lead levels in the 2nd trimester of pregnancy. Our patient's lead levels dropped significantly after chelation, 
and her anemia improved significantly. The effect of DMSA on fetal lead concentrations is still unclear. Our patient continues to be followed longitudinally with plans to check a neonatal lead level upon delivery, and initiate chelation, if necessary. The target lead level for cessation of chelation therapy in this patient remains unclear.

156. 38 Weeks Pregnant: Managing a Mother and Neonate After a Third-Trimester Acetaminophen Ingestion

Charlotte E. Goldfine ${ }^{1}$, Michael Ganetsky ${ }^{2}$

${ }^{1}$ Brigham and Women's Hospital, Boston, MA, USA. ${ }^{2}$ Beth Israel

Deaconess Medical Center, Boston, MA, USA

Background: Although acetaminophen (APAP) is the most common drug overdose in pregnancy, data is limited regarding the management of a neonate who is delivered shortly after the mother overdosed on APAP.

Hypothesis: We report the case of a young woman who had an emergency cesarean section after presenting with an overdose of APAP and quetiapine.

Results: A 25-year-old woman at 38 weeks of gestation was brought to the emergency department (ED) after she texted a friend suicidal statements. She reported taking an unknown amount of quetiapine. In the ED, she became somnolent and hypoxic, requiring intubation. Laboratory examination was significant for APAP level of $68 \mathrm{mcg} / \mathrm{mL}$, AST 17 , ALT 10, and urine toxicology screen positive for benzodiazepines, tricyclic antidepressants, and buprenorphine. Patient was started on nacetylcysteine (NAC). She started having contractions and the decision was made to perform an emergent cesarean section. Prior to delivery, the patient had received $150 \mathrm{mg} / \mathrm{kg}$ NAC over 60 minutes. The infant was delivered without complication. Due to a laboratory issue, an APAP level was unable to be obtained on the infant immediately so was treated with $\mathrm{NAC}$ at $15 \mathrm{mg} / \mathrm{kg} / \mathrm{hr}$ in sterile water. An APAP level 7-hours after birth in the infant was $13 \mathrm{mcg} / \mathrm{mL}$ with an AST 28 and ALT 6. The infant was continued on NAC at the same dose until 21.75 hours post-birth with an undetectable APAP level. The infant never developed any signs of hepatotoxicity. The mother was also continued on NAC protocol and was able to be extubated. She continued to improve until discharge to a psychiatric facility and never developed hepatotoxicity.

Conclusion: APAP overdose requiring treatment in both a mother and neonate is rare but can be successfully treated with NAC. Given differences in neonatal pathophysiology, consideration was taken for amount of dextrose and volume.

157. Case Report of Intrauterine Nadolol Exposure Associated with Small for Gestational Age, Cardiorespiratory Distress, and Hypoglycemia

Nohea L Leatherman-Arkus, Cherie Obilom, Ameet Thaker, Dazhe James Cao

UT Southwestern, Dallas, TX, USA

Background: The prevalence of hypertension affecting pregnancies is on the rise, and beta-blockers are the most prescribed class of cardioactive medications in pregnancy. Although some adverse effects common betablockers used in pregnancy are described, little is known of the potential adverse effect of nadolol on the developing human fetus in utero and in the immediate neonatal period.

Hypothesis: Perinatal maternal nadolol exposure restricts fetal growth and is associated with neonatal cardiopulmonary distress and hypoglycemia.

Methods: This is a single-patient chart review. A female infant was born at 33-week gestation for intrauterine growth restriction, oligohydramnios, and a category II fetal heart tracing. The mother was a 19-year-old with a history of catecholaminergic polymorphic ventricular tachycardia and was stabilized on $40 \mathrm{mg}$ of nadolol daily for three years prior to and throughout the pregnancy. The infant was born small for gestational age between the 3rd and 10th percentiles for weight and body mass index. The placenta was less than the 10th percentile for gestation.

Results: In the first 24 hours of life, this infant had clinically significant bradycardia (heart rate ranged $110-130$ beats per minute) with intermittent oxygen desaturations and agitation. She was treated with continuous positive airway pressure and intravenous caffeine $(20 \mathrm{mg} / \mathrm{kg}$ once $)$ and weaned off to room air by 48 hours of life. Additionally, hypoglycemia (nadir of $43 \mathrm{mg} / \mathrm{dL}$ ) was noted that resolved with parenteral nutrition infusion. Nadolol exposure was confirmed by cord blood $(37 \mathrm{ng} / \mathrm{mL})$.

Conclusion: Beta-blocker use during pregnancy has been associated with growth restriction, neonatal hypoglycemia, and respiratory depression. Nadolol is a long-acting non-selective beta-blocker capable of crossing the placental barrier and into breast milk and may have more profound effects to a developing fetus and neonate. Alternatives to nadolol should be considered during pregnancy and lactation.

\section{Comparison of Poisoning Exposures in Pregnant and Non-} pregnant Women

James B Leonard, Faisal Syed Minhaj, Emily Paterson, Wendy KleinSchwartz

University of Maryland School of Pharmacy, Baltimore, MD, USA

Background: Poisoning is an important cause of maternal harm during pregnancy. Current research on poisoning in pregnancy is limited to only pregnant subjects and there is a need for research utilizing non-pregnant control groups.

Research question: How do poisonings in pregnant women differ from non-pregnant women?

Methods: This was a retrospective study utilizing the National Poison Data System (NPDS) from 2000 to 2019. The NPDS was queried for all calls about pregnant women from the age of 15 to 44 years and a 5:1 agematched cohort of non-pregnant controls (NPC). Characteristics and outcomes within the cohort were described.

Results: There were 131,619 pregnant cases (PC) (2.4\% of all female NPDS cases); median age 27 (IQR: 22, 31). Median age for all females between 15 to 44 years in NPDS $(n=5,494,808)$ was 26 (IQR: 19, 35). While median ages were similar, the distributions of ages were different. In PC, $15-19$-year-olds made up $11.6 \%$ of the cohort compared with $24 \%$ of all females in NPDS. PC were agematched to 658,095 NPC. Intentional exposures were more common in NPC $(41.2 \%)$ than PC $(21.9 \%)$. There was an increase over time in self-harm attempts for NPC $\left(R^{2}=0.9177\right)$, but not for PC $\left(R^{2}=\right.$ $0.1458)$. There were fewer environmental exposures in NPC $(3.8 \%)$ than PC (12.1\%). Fumes, gases, and vapors were less common with NPC $(2.7 \%)$ vs. PC $(6.6 \%)$. Analgesics were more common in NPC $(17.4 \%)$ than PC $(11.9 \%)$. Moderate and major effects were more frequent in NPC $(14.3 \%)$ than PC $(7 \%)$.

Conclusion: Poisoning exposures in pregnant patients make up a small proportion of female cases reported to U.S. poison centers. Exposures in pregnant patients are less likely to be intentional and more likely to be environmental than those in non-pregnant controls. Many reported exposures in pregnant patients are benign and outcomes are less severe than those in non-pregnant controls.

\section{Observations Impacting Pregnancy in the ToxIC Registry}

Kaytlin E Hack ${ }^{1,2}$, Maryann Mazer-Amirshahi ${ }^{2,3}$, Sharan Campleman ${ }^{4}$, Dana Karshenas ${ }^{4}$, Anthony F Pizon ${ }^{5}$, On Behalf of the ToxIC Investigators Consortium (ToxIC) 
${ }^{1}$ MedStar Georgetown University Hospital, Washington, DC, USA. ${ }^{2}$ MedStar Washington Hospital Center, Washington, DC, USA. ${ }^{3}$ Georgetown University School of Medicine, Washington, DC, USA. ${ }^{4}$ American College of Medical Toxicology, Phoenix, AZ, USA.

${ }^{5}$ University of Pittsburgh School of Medicine, Pittsburgh, PA, USA.

Background: Many xenobiotics are well known to have consequences on both pregnant women and developing fetuses, including teratogenicity, placental abruption or pregnancy loss.

Research Question: How frequently are women of child-bearing age who are evaluated by a medical toxicologist receiving a pregnancy test? Additionally, which agents are most commonly implicated in toxic exposures in women of child-bearing age?

Methods: This is a retrospective study of consecutive patients who identify as women from the Toxicology Investigators Consortium (ToxIC) registry from 2018 through 2019. A search of the ToxIC registry of all entries for women of child-bearing age, defined as age 12 to 55 years, was analyzed.
Results: Women of child-bearing age made up $95.4 \%$ of patients who identified as women of all documented cases reported to the registry $(1150 / 1205)$. A pregnancy test was documented in $75.6 \%$ of cases reported to the registry $(838 / 1124)$. The most common toxins reported in women of child-bearing age were in descending order: analgesics (20\%), antidepressants $(13 \%)$, unknown agents $(13 \%)$, opioids $(10 \%)$, sedative/ hypnotic medications $(6 \%)$, and alcohol (5\%). Several known teratogens made up $11 \%$ of toxic exposures which include alcohol, lithium, anticoagulants, anticonvulsants, metals, and chemotherapeutic or immunomodulating agents. Sixty-five percent (752/1150) of women in the registry received treatment as a result of medical toxicology consultation. Additionally, 17\% (198/1150) and 34\% (392/1150) of this patient population were screened by medical toxicologists for alcohol and opioid use, respectively.

Conclusion: Women of child-bearing age who present with toxic ingestions are tested for pregnancy a majority of the time, but surprisingly nearly $25 \%$ are not tested for pregnancy. There is also significant opportunity to increase the rates of screening for alcohol and opioid use in women of child-bearing age.

\section{ToxIC}

This research was performed in collaboration with the ACMT Toxicology Investigators Consortium.

160. Fatality Trends Using the Toxicology Investigators Consortium (ToxIC) Registry: a 6-year Review of Demographics and Clinical Characteristics

Jennifer S Love ${ }^{1}$, Kim $\mathrm{Aldy}^{2}$, On Behalf of the ToxIC Investigators Consortium (ToxIC) ${ }^{3}$

${ }^{1}$ Oregon Health \& Science University, Portland, OR, USA. ${ }^{2}$ University of Texas Southwestern Medical Center, Dallas, TX, USA

Background: Unintentional poisoning deaths are the leading cause of injury-related mortality in the United States. However, little is known about demographic trends and clinical characteristics of deaths related to poisonings/toxicologic exposures.

Research Question: Have demographic and clinical characteristics of toxicologic deaths entered into the ToxIC database changed from 2014 to 2019 ?

Methods: This is a retrospective review of ToxIC Registry cases with a fatal outcome between 2014 and 2019. Cases were compared by year with a focus on demographics, exposure, intent, and clinical characteristics. Descriptive statistics and comparisons were gathered using SPSS. Results: Between 2014 and 2019, 616 deaths were reported. Total annual fatalities ranged from 89 to 128 . Most deaths (70-75\%) occurred in adults age 19-65 years old across all years, except in 2019 (58\% deaths). Deaths in patients $<18$ years old accounted for $10-15 \%$ most years, except in 2019 (22\% deaths). Deaths were generally split evenly across all genders (49\% males) each year. Race and ethnicity was comprised of $58.1 \%$ White, $12.5 \%$ Black, and $9.9 \%$ Hispanic. The top three reasons for toxicologic exposure included intentional use of pharmaceutical product (49.9\%), intentional use of a non-pharmaceutical product $(15.1 \%)$, and organ system dysfunction (14.4\%). The top three primary exposure agent classes included analgesics (17.4\%), opioids (14.3\%), and cardiovascular $(9.3 \%)$. The leading reasons for fatal toxicologic exposures remained relatively constant over time. However, the primary agents showed some variation, with opioid and non-opioid analgesics varying as the leading agent over time.

Conclusion: The number of fatal cases seen by medical toxicologists has remained relatively constant over six years. Most fatal cases are adult age and have an intentional exposure to a pharmaceutical product, which is commonly an opioid or non-opioid analgesic. Understanding the evolving demographics and clinical characteristics of fatal toxicologic exposures can inform targeted public health messaging and interventions around poisonings.

\section{ToxIC}

\section{This research was performed in collaboration with the ACMT Toxicology} Investigators Consortium.

161. Treatment Modality Trends in Fatalities Reported to the Toxicology Investigators Consortium (ToxIC) Registry: a Six Year Review

Jennifer S Love ${ }^{1}$, Kim $\mathrm{Aldy}^{2}$, On Behalf of the ToxIC Investigators Consortium (ToxIC)
${ }^{1}$ Oregon Health \& Science University, Portland, OR, USA. ${ }^{2}$ University of Texas Southwestern Medical Center, Dallas, TX, USA

Background: Unintentional poisoning deaths are the leading cause of injury-associated mortality in the United States. Given their illness 
severity, patients who die from poisonings may receive various critical care treatments. Increased accessibility of critical care training and interventions may affect trends in critical care modalities and antidotes used to treat critically ill toxicologic patients.

Research Question: For patients with fatal overdose deaths, have intensive care treatment modalities or antidotes changed from 2014 to 2019? Methods: This is a retrospective review of ToxIC Registry cases with a fatal outcome between 2014 and 2019. Cases were compared by year with attention to critical care treatment modalities and antidote use. Descriptive statistics and comparisons were performed using SPSS.

Results: Six hundred and sixteen deaths occurred over six years. Total annual fatalities ranged from 89 in 2014 to 128 in 2016. Life support was withdrawn in $64 \%$ of patients, and brain death was confirmed in $26 \%$ of patients in whom life support was withdrawn. Common interventions included intubation/ventilatory support (57\%), intravenous fluids
(54\%), vasopressors (51\%), N-acetylcysteine (25\%), CPR (21\%), benzodiazepines (20\%) and sodium bicarbonate (19\%). Critical care antidotes including methylene blue (2.8\%), hydroxocobalamin (3.4\%), insulin euglycemic therapy $(5.8 \%)$ and lipid rescue $(6.3 \%)$ were used infrequently. However, use of these therapies nearly doubled from 2014 to 2015. Continuous renal replacement therapy (CRRT) use was more common than hemodialysis (HD) (15\% vs. $3.1 \%$ or $6.8 \%)$. There was an increase in HD use for toxin removal and CRRT in 2016. ECMO was used in $3.2 \%$ of fatalities.

Conclusion: Critical care treatments in patients with fatal toxicologic exposures demonstrate variability and reflect the suspected xenobiotic (i.e., 25\% received NAC). Other treatment modalities such as methylene blue or lipid rescue therapy are not frequently used. HD and CRRT use increased in 2016, which correlates with the release of EXTRIP guidelines in 2015.
162. Gastrointestinal Decontamination: Trends from the ToxIC Case Registry, a Five-Year Review

Brian G Lewis, Bryan S Judge, On Behalf of the ToxIC Investigators Consortium (ToxIC)

Spectrum Health, Grand Rapids, MI, USA

Background: Gastrointestinal (GI) decontamination following toxicologic exposures has largely fallen to the wayside over the past several decades. However, a variety of GI decontamination methods remain in use for some toxic exposures.

Research Question: For which toxicologic exposures was GI decontamination utilized over a five-year period in patients registered in the ToxIC Case Registry?

Methods: This retrospective database review using the ToxIC Case Registry from 2014 to 2019 , examined the overall rate and specific methods of GI decontamination as they relate to patient age, toxicologic exposure, presenting signs and symptoms, and need for additional treatment.
Results: The overall utilization rate for GI decontamination during the study period was $3.34 \%$. Activated charcoal was the most common method of GI decontamination $(3.04 \%)$ in all age groups, and was most commonly used for analgesic, cardiovascular, and antidepressant medication exposures. Less commonly employed methods were whole-bowel irrigation $(0.38 \%)$ and gastric lavage $(0.13 \%)$. Rates of GI decontamination in pediatric patients less than seven years of age $(4.21 \%)$ and between seven and 18 years of age $(5.73 \%)$ were significantly higher than patients 18 years of age and older $(2.78 \% ; p<0.0001)$. Over $15 \%$ of patients receiving all forms of GI decontamination presented with tachycardia (>140 BMP) and greater than $49 \%$ of patients had neurologic symptoms. Fourteen of the 1593 patients receiving GI decontamination died.

Conclusion: Gastrointestinal decontamination is used more commonly in pediatric patients than adult patients. Further study of certain toxicologic exposures receiving GI decontamination, specifically activated charcoal, is needed to determine the presence or absence of improved patient outcomes.

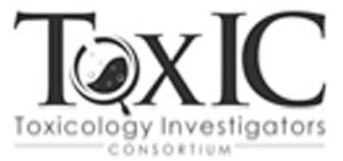

\section{This research was performed in collaboration with the ACMT Toxicology Investigators Consortium.}

\section{Logistical Holes in Whole Bowel Irrigation}

Kyle P Suen ${ }^{1,2}$, Andres Guzman-Soto ${ }^{1,2}$, Daniel Nogee ${ }^{1,2}$, Patrick Filkins $^{2}$, Connor Walsh ${ }^{2}$, Joseph Carpenter ${ }^{1,2}$

${ }^{1}$ Emory School of Medicine, Department of Emergency Medicine, Division of Medical Toxicology, Atlanta, GA, USA. ${ }^{2}$ Georga Poison Center, Atlanta, GA, USA

Background: Whole-bowel irrigation (WBI) is used as a method of gastrointestinal decontamination for poisoned patients, but may have low compliance by providers when recommended by poison centers
(PC). As part of a multi-stage project aimed at improving the quality of WBI recommendations from our PC, we set out to determine WBI compliance when recommended, as well as analyze temporal trends and a possible association with outcomes.

Methods: We conducted a retrospective chart review of data from 1/1/ 2010 to 12/31/2019 within our PC. We included all patients aged $>2$ who had WBI recommended and were followed to a known outcome. We collected demographic data, exposure history, therapies recommended and performed, and clinical outcomes. Data were analyzed to evaluate compliance rate of WBI. Chi-squared and Mann-Whitney $U$ tests were performed to look for any differences in compliance based on age, 
gender, or year, and to determine if WBI compliance was associated with a change in clinical outcome.

Results: Four hundred and eighty-three cases met inclusion criteria. Fifty-five percent of patients were male and median age was 32.5 years. WBI was reportedly performed in 281 cases for a compliance rate of $58.2 \%$. The most common ingestions for which WBI was recommended were calcium channel blockers, beta blockers, lithium, benzodiazepines, cocaine, and amphetamine products. There were no differences in compliance when considering age, gender, or year $(p=\mathrm{NS})$. Finally, there was no difference between whole bowel irrigation therapy and clinical outcomes $(p=\mathrm{NS})$.

Conclusion: WBI compliance at this single $\mathrm{PC}$ is lower than nationallevel data for other therapies recommended by PCs. We plan to further analyze the case data, including narratives, and to perform prospective data collection on reasons why hospitals do not perform WBI when recommended.

\section{Severe Perampanel Toxicity in a Pediatric Patient}

Garret A Winkler, Alicia B Minns, Allyson A Kreshak

University of California San Diego, San Diego, CA, USA

Background: Perampanel (Fycompa ${ }^{\circledR}$ ) is a new antiepileptic used to treat partial seizures and generalized tonic-clonic seizures in people older than 12 years old. Perampanel is a selective non-competitive AMPA receptor antagonist with a prolonged half-life of approximately $105 \mathrm{hrs}$. Few cases of significant toxicity have been reported in the literature and effects in overdose are poorly understood.

Methods: This is a single-case report of a pediatric patient treated after an unintentional, exploratory ingestion of perampanel. A 20-month-old, otherwise healthy female ingested eight mg of perampanel, her mother's medication. The patient presented to a pediatric emergency department one hour after ingestion with ataxia, irritability, and somnolence. Vital signs were HR $130 \mathrm{bpm}$, BP 112/97 mmHg, temp $99^{\circ} \mathrm{F}$, RR 30. The patient was admitted for observation to the pediatric intensive care unit. Results: During the hospitalization, the patient developed persistent bradycardia to the 70s with lowest recorded HR of $71 \mathrm{bpm}$, which improved with stimulation alone. Bradycardia persisted until hospital day hospital day (HD) three. Physical examination was notable for somnolence and truncal ataxia with irritability when aroused, which improved throughout the hospitalization. On HD three, the patient exhibited an ataxic gait but otherwise normal mental status. A quantitative level obtained on HD three was $750 \mathrm{ng} / \mathrm{ml}$. In adults, a peak concentration after a single $12 \mathrm{mg}$ dose averaged $800 \mathrm{ng} / \mathrm{ml}$. On HD three, the patient was noted to be ataxic but otherwise had an age-appropriate neurologic examination. The patient was discharged on HD four with normal vital signs, return to baseline mental status, and baseline gait.

Conclusions: Toxicity from a perampanel overdose is poorly understood both in adults and pediatric patients. This pediatric patient's toxicity was characterized by bradycardia, somnolence, agitation, altered mental status, and ataxia that resolved with supportive care which is consistent with prior case reports.

\section{Pediatric Nifedipine Overdose with Survival after Cardiac Arrest}

Richard McNulty ${ }^{1,2}$, Nathan Lieu ${ }^{3}$, Natalie Duns ${ }^{3}$, Elena Cavazzoni ${ }^{3}$, Chong Tien $\mathrm{Goh}^{3}$

${ }^{1}$ Blacktown Mount Druitt hospital, Sydney, NSW, Australia. ${ }^{2}$ Western Sydney Toxicology Service, Sydney, NSW, Australia. ${ }^{3}$ The Children's Hospital at Westmead, Sydney, NSW, Australia

Background: Pediatric survival after cardiac arrest from nifedipine, a dihydropyridine $\mathrm{Ca}$-Channel Blocker (CCB), is uncommonly reported.
Hypotheses: Return to baseline function is possible after prolonged cardiac arrest in pediatric nifedipine overdose. In addition to vasodilation, nifedipine toxicity can be associated with poor contractility.

Methods: This is a single-patient chart review with family consent.

Results: A 10-month-old, 10-kg baby had a background of West syndrome and hydrocephalus. Clinical baseline included movement disorder and autonomic dysfunction. While recovering in hospital after a VP shunt revision, he was accidentally given nifedipine $90 \mathrm{mg}$. Hypotension developed within 40 minutes, progressing to witnessed cardiac arrest and immediate CPR. Rhythm varied from asystole to PEA. Initial treatment included: adrenaline $100 \mathrm{mcg} \times 11$ doses; calcium chloride $10 \% 2 \mathrm{~mL}$ IV $\times$ 4; High dose Insulin Euglycaemic Therapy (HIET: 10 units insulin IV followed by infusion); and sodium bicarbonate $10 \mathrm{mmol}$. ROSC was achieved after 42 minutes and activated charcoal was given after intubation. Blood gasses showed a $\mathrm{pH}$ nadir of 6.38 during arrest, bicarbonate $5.2 \mathrm{mmol} / \mathrm{L}$, and ionized calcium $3.47 \mathrm{mmol} / \mathrm{L}$. ECG: sinus rhythm with no blocks. HIET was continued because initial ECHO indicated cardiogenic shock, but was ceased after 10 hours as contractility became hyperdynamic. Hypotension persisted 24 hours despite adrenaline, noradrenaline, vasopressin, and methylene blue. Vasopressors were weaned on day three. Extubation occurred after one week and the child was eventually discharged at previous baseline neurological function. Although no serum nifedipine concentrations were obtainable, the unique circumstances of the case confirm nifedipine as the cause.

Conclusion: Prompt CPR, advanced life support and antidotal therapy was associated with a good outcome in this CCB-induced cardiac arrest. Reduced contractility in this case was perhaps multifactorial due to CPR, acidaemia, and nifedipine. A possible role for HIET in severe dihydropyridine $\mathrm{CCB}$ overdose needs more study.

166. Critical Illness from an Exploratory Ingestion of a So-Called "Homeopathic" Remedy Mailed from Pakistan: Caffeine / Yohimbine Poisoning of a Young Child

Kevin C Osterhoudt ${ }^{1}$, Michael Demarco ${ }^{2}$, Joan Nolan ${ }^{1}$, Brian Singh ${ }^{3}$, Leslie M Shaw ${ }^{4}$, Justin L Lockman ${ }^{5}$

${ }^{I}$ The Poison Control Center at Children's Hospital of Philadelphia, Philadelphia, PA, USA. ${ }^{2}$ Department of Emergency Medicine, Crozer Health, Drexel Hill, PA, USA. ${ }^{3}$ Clinical Laboratory, Children's Hospital of Philadelphia, Philadelphia, PA, USA. ${ }^{4}$ Pathology and Laboratory Medicine, Hospital of the University of Pennsylvania, Philadelphia, PA, USA. ${ }^{5}$ Critical Care Medicine, Children's Hospital of Philadelphia, Philadelphia, PA, USA

Background: Vitamin supplements, natural remedies, homeopathic elixirs, and ayurvedic preparations are not regulated in the manner of pharmaceuticals. Problems with purity, mislabeled or unlabeled contents, and contaminants have been frequently encountered problems leading to toxic health events. We observe that global web-based, mail-order sales of unregulated medical products adds complexity to the toxicological assessment of such exposures, and that poisonings with such products continue to occur.

Methods: We report a case of a 14-month-old boy who was found to have vomited two white pills. These pills were recognized by his father as a "homeopathic" medicine, mailed from an online homeopathic doctor in Pakistan, ostensibly used to supplement care of the father's diabetes (photos available). The boy quickly became tremulous, suffered refractory seizures, and was endotracheally intubated for respiratory compromise. The prescribing physician was called and he believed the pills to be methylcobalamin.

Results: In the hospital, the boy had a heart rate of $210 / \mathrm{min}$, blood pressure $120 / 56 \mathrm{mmHg}$, and temperature $38.0 \mathrm{C}$. Laboratory evaluation was notable for metabolic acidosis and a serum potassium of $2.7 \mathrm{meq} / \mathrm{L}$. An ECG showed sinus tachycardia. The boy recovered from illness, and returned to good health, with intensive supportive care. GC/MS analysis 
of the boy's urine found caffeine, theophylline, theobromine, ephedrine, and yohimbine. GC/MS analysis of one of the recovered pills found caffeine, ephedrine, and yohimbine.

Conclusion: The content of these pills from an international, online, mail-order homeopath were not labeled on the bottle, and turned out to be different than the prescribing doctor believed. Toxic syndrome consideration deduced caffeine and/or theophylline poisoning, and this was confirmed by laboratory analysis. Previous reports have documented the potential danger of caffeine and/or yohimbine in pediatric exploratory poisoning, and this case adds to the accumulated experience with this poisoning syndrome.

\section{Ingestion of Naturopathic Wart Removal Agent in Infant} Resulting in Hematemesis and Shock

Christopher N Sweat ${ }^{1,2}$, Alexandru Ulici ${ }^{1}$, Travis D Olives ${ }^{1,3}$

${ }^{1}$ Minnesota Poison Control System, Minneapolis, MN, USA. ${ }^{2}$ Regions Hospital, Saint Paul, MN, USA. ${ }^{3}$ Hennepin Healthcare, Minneapolis, MN, USA

Background: Commonly available over the counter wart removal agents include keratolytics such as salicylic acid and blistering agents such as cantharidin, a terpenoid produced by beetles of the Meloidae family. Less common agents such as immunomodulators and topical chemotherapeutics are available with prescriptions for refractory cases.

Methods: This is a single-patient case report. A three-month-old boy presents to the ED after being fed a naturopathic wart removal agent by his older sibling and developing hematemesis, arriving hypoxemic and in shock.

Results: On arrival, he was hypotensive to $60 / 40$ with $\mathrm{SpO} 2<80 \%$, requiring intubation and fluid resuscitation. Initial labs were notable for a negative salicylate, iron level of 95, WBC count of 33,000, and hemoglobin of $11 \mathrm{~g} / \mathrm{dL}$ which dropped to 8.2 on serial assessment. The substance was presented as an unlabeled 2-3-ounce glass vial containing traces of a clear yellow liquid, purchased as a wart remover from a natural healing shop. Within two hours of presentation, the patient underwent EGD which demonstrated diffusely blackened, friable tissue of the entire esophagus, stomach, and visualized portions of the duodenum in addition to edema and blistering of the pharynx and trachea. A nasogastric tube was placed and he was administered PRBCs, steroids, and antibiotics, then admitted and started on TPN. Over the subsequent days, he underwent serial endoscopies demonstrating mucosal sloughing without evidence of perforation or fistula. He was extubated approximately 48 hours after arrival, at which time he was noted to have facial blisters concerning for exposure to cantharidin. He remained hospitalized for a total of three weeks, discharging home after transition from TPN to tube feeds.

Conclusion: Ingestion of topical wart removers, particularly cantharidin, may result in catastrophic corrosive injury. Given blistering and mucosal sloughing, we suspect exposure to cantharidin. Definitive identification is pending through the department of health.

\section{Tetrahydrocannabinol Induced Status Epilepticus in a 13- Month-Old}

Sam T Ontiveros, Bryan Corbett

University of California San Diego, San Diego, California, USA

Background: The recreational sale and use of tetrahydocannabinol (THC) containing products was legalized in California on November 8 , 2016. Pediatric exploratory ingestions of THC have subsequently risen. Methods: This is a single-patient chart review. A 13-month-old healthy female with an unremarkable birth history and no family history of seizures was found somnolent by her mother and brought to the Emergency
Department. On arrival, myoclonic movements and eye fluttering concerning for ongoing seizure were noted and intravenous lorazepam was administered at $0.1 \mathrm{mg} / \mathrm{kg}$ without effect. A second dose was administered and symptoms resolved. A Levetiracetam load was administered and the patient was admitted to the pediatric intensive care unit. The patient's mental status improved slightly over the next hour, but remained very depressed. No focal neurologic deficits were noted on exam. The initial workup included a non-contrast CT head, a complete blood count, and a complete metabolic panel which were all unremarkable. Acetaminophen and salicylate levels were undetectable. However, the patient's urine drug screen was positive for cannabinoids. On searching the house, the patient's father found an empty cardboard container that had contained a small edible THC gummie. The label listed a dose of 600 mg.

Results: The patient's mental status slowly improved, but remained depressed for 48 hours. At discharge, the patient had returned to baseline. She had no further seizures.

Conclusion: THC containing edible products are now readily available at high concentrations. Serious adverse effects, including convulsions, may be seen when ingested by children.

\section{Pediatric Benzodiazepines Exposures Reported to the U.S. Poison Centers}

Saumitra Rege, Sarah Ames, Heather Borek, Jennifer Ross, Christopher Holstege

University of Virginia, Charlottesville, VA, USA

Background: The rate of visits involving benzodiazepine prescriptions increased from 3.8 to $7.4 \%$ between 2003 and 2015 in the United States. Benzodiazepine-associated overdose mortality has risen sharply, from 0.6 per 100000 adults in 1999 to 4.4 per 100000 in 2016 . The objective of the study was to describe the epidemiology of pediatric benzodiazepines exposures using a near real-time national poison center (PC) database.

Methods: The National Poison Data System (NPDS) was queried for pediatric (0-19 years) exposures to benzodiazepines from 2011 to 2019 using generic code identifiers. We descriptively assessed the relevant demographic and clinical characteristics. Trends in benzodiazepines frequencies and rates (per 100,000 human exposures) were analyzed using Poisson regression methods. Logistic regression was used to identify important predictors of severe health outcomes (SHO), defined as cases resulting in major medical outcomes or death.

Results: There were 133,704 pediatric exposures to benzodiazepines reported to the PCs from 2011 to 2019, with the number of calls decreasing from 16,830 to 11,776 during the study period. Polysubstance exposures accounted for $41.1 \%$ of pediatric benzodiazepines exposures. The residence was the most common site of exposure (93.6\%) and 52.2\% of cases were females. Suspected suicides $(33.6 \%)$ was the most commonly reported reason for exposure, attributed to the teenage population. There were 3,209 cases of $\mathrm{SHO}$, with these exposures increasing by $45.2 \%$ during this period (334 to $485, p<0.001$ ). This risk of SHOs increased with age, with cases between 6 and 19 years (adjusted odds ratio $\{A O R\}$ : 3.41, 95\% CI: 2.93-4.09) demonstrating significantly increased odds. Cases of suspected suicide (AOR: 1.19, 95\% CI: 1.07-1.34) and abuse (AOR: 1.49, 95\% CI: 1.31-1.69) significantly resulted in SHO.

Conclusion: Benzodiazepine exposures reported to poison centers decreased during the study period. This risk of benzodiazepine-associated SHOs increased overall during the study period.

\section{Constipation Remains One of the Earliest Clinical Signs of Infantile Botulism}

Stephanie L Widmer, Wells Brambl, Joshua Nogar

Northwell Health, Manhasset, NY, USA 
Background: Infantile botulism is the most common form of botulism in the United States. It tends to occur in infants less than six months of age and often presents with difficulty feeding and decreased muscle tone, with constipation being one of the earliest symptoms. We report a case of infantile botulism type-B in New York City.

Methods: A 5-month-old female re-presented to the Emergency Department (ED) for decreased muscle tone, poor suck, and difficulty feeding just one day after an admission for constipation and dehydration. On return visit, the patient was unable to hold her head up. The baby had not stooled for the past six days. The physical exam was notable for normal vitals, with significant hypotonia, weak cry, absent suck reflex and gag, and absent deep tendon reflexes. She was placed on continuous end-tidal $\mathrm{CO} 2$, made NPO, and treated with empiric baby botulinum immunoglobulin (BabyBIG). Stool studies were ultimately positive for Clostridium botulinum B, which is known to be the more common type found east of the Mississippi. The rest of the workup, including lumbar puncture, MRI, metabolic labs, and karyotype were unremarkable. The source of our patient's infection remains unclear, although her mother reports the use of a chamomile containing herbal remedy for colic, and a potential exposure to store-bought honey. Throughout hospital stay, patient demonstrated improvement with tone, suck, and DTRs from initial presentation. By day eight, patient was tolerating $\mathrm{PO}$ and was discharged home on day 11 .

Conclusion: Botulism is a potentially fatal illness for which patient survival depends on early diagnosis and antitoxin procurement. Constipation remains one of the earliest signs of infantile botulism and is a symptom that should provoke consideration of this diagnosis.

171. Comparison of the Prevalence of Pediatric Poisonings Before, During and After COVID-19 Shelter-in-Place

Thao-Phuong C Hallett ${ }^{1}$, Allen M Hallett ${ }^{2}$, Rebecca E Bruccoleri ${ }^{3}$ ${ }^{1}$ Monroe Carell Jr. Children's Hospital at Vanderbilt, Nashville, TN, USA. ${ }^{2}$ The University of Texas Health Science Center at Houston, School of Public Health, Austin, TX, USA. ${ }^{3}$ Tennessee Poison Center, Nashville, TN, USA

Background: Poison exposures most often occur at home but there may be changes in the prevalence of poisoning among children when all individuals were ordered to stay home due to the COVID-19 pandemic.

Research Question: Does the prevalence of reported pediatric poisoning change during the time period surrounding the COVID-19 shelter-inplace?

Methods: This is a retrospective serial cross-sectional study of Tennessee children with known age of 0-17 years. On March 30, 2020, an executive order was placed by the Governor of Tennessee for a safer-at-home order but shifted toward a shelter-in-place order on April 2, 2020, that lasted until April 30, 2020. We obtained counts and demographic information from calls to the Tennessee Poison Center about poison exposures in children from before (March 2020), during (April 2020), and after (May 2020). We subtracted 2020 Census estimates of the resident population aged $>18$ years from the total resident population in Tennessee to calculate the total number of children aged $<18$ years. Aggregate call count and population data were used to derive the prevalence estimates of poisoning for each month. The chi-square test was applied. Statistical significance was set at $p<0.05$.

Results: Tennessee prevalence estimates of poison exposures among children was $0.147 \%$ in March 2020, $0.139 \%$ in April 2020, and $0.151 \%$ in May 2020 . There was statistically significant evidence of association between reported poisoning exposure and month $\left(\chi^{2}=\right.$ $7.59 ; p=0.023$ ).

Conclusion: The prevalence of reported pediatric poisoning was associated with timing of the COVID-19 shelter-in-place order between March and May 2020. Additional evaluation of epidemiological trends, time points, confounders such as parental or caregiver presence, and sources of poisoning among children surrounding the COVID-19 shelter-in-place is warranted for timely prevention and management.

172. Did the COVID-19 'Lockdown' in Melbourne, Australia Influence Adolescent Deliberate Self-Poisoning (DSP) and MentalHealth Presentations to a Metropolitan Hospital Network?

Nicola Rodd ${ }^{1}$, Andis Graudins ${ }^{1,2}$

${ }^{1}$ Monash Health, Melbourne, Victoria, Australia. ${ }^{2}$ Monash University, Clayton, Victoria, Australia

Background: The COVID-19 pandemic in Melbourne, Victoria, Australia, led to a several-month lockdown and consequent widespread social isolation.

Hypothesis: The COVID-19 pandemic and associated lockdown led to an increase in both adolescent deliberate self-poisoning (DSP) and mental health presentations to a metropolitan health network.

Methods: Retrospective comparison of adolescents (age 10-18 years) presenting to a metropolitan health network with DSP or mental health problems during matched periods of March to September 2019 and 2020. Presentations were identified by searching the hospital electronic medical record and cross-referenced with the toxicology referral database. Data extracted: demographics, triage reason (DSP or mental health), substances ingested, ED disposition, hospital length of stay (LOS).

Results: Total adolescent ED presentations in the seven-month periods were 10,055 in 2020 and 14,023 in 2019. There were 199 DSP presentations in each period. This constituted a greater proportion of ED presentations in 2020 ( $2 \%$ vs. $1.4 \%$, OR: $1.4,95 \%$ CI: $1.1-1.7, p=0.0009$ ). Median age was 16 years (IQR 15-17) for both time periods. Mental health presentations were also greater in 2020 (16\% vs. 10\%, OR: 1.6 95\% CI: $1.2-3.5, p<0.0001)$. There was a significant increase in DSP requiring ICU and medical-ward admission in 2020: medical-ward (23\% vs. $8.0 \%$, OR:3.5, 95\% CI: $1.9-6.3, p=<0.0001)$, ICU (9.5\% vs. $1.0 \%$, OR: 10.4, 95\% CI: 2.65-45.53, $p=0.0002)$, and an increase in DSP with sedative drugs (benzodiazepines and other sedatives) $(16.2 \%$ vs. $5.2 \%$, OR: $3.5,95 \%$ CI: $1.9-6.4, p=<0.0001)$, and concomitant decrease in DSP with antidepressants ( $12.4 \%$ vs. $23 \%$, OR: $0.48,95 \%$ CI: $0.30-0.75$, $p=<0.0015)$. Acetaminophen was the most commonly reported toxicant for DSP (30\%) in both time periods. There was no difference in gender (2019: $83 \%$ vs. 2020 : $76 \%$ female), ED-LOS, frequency of psychiatric admission.

Conclusion: An increase in adolescent-DSP and mental health presentations compared to total ED presentations was observed in 2020, as well as more ICU and medical-ward admissions, suggesting an increased rate of more significant intoxications in 2020.

173. Did the COVID-19 'Lockdown' in Melbourne, Australia Influence Deliberate Self-Poisoning (DSP) and Mental Health Presentations in Older Adults (> 60)?

Joel Mun Hoe Kuan ${ }^{1}$, Andis Graudins ${ }^{1,2}$

${ }^{1}$ Monash Health, Melbourne, Victoria, Australia. ${ }^{2}$ Monash University, Clayton, Victoria, Australia

Background: The COVID-19 pandemic in Melbourne, Australia, led to a lockdown for several months and consequent widespread social isolation.

Hypothesis: That the COVID-19 pandemic and associated lockdown led to a change in both older adult DSP and mental health presentations to a major metropolitan health network.

Methods: Retrospective comparison of older adults ( $>60$ years) presenting to our health service with DSP or mental health problems during matched periods of March-September 2019 and 2020. Presentations were identified by searching the hospital electronic medical record and 
included if final diagnosis was "deliberate self-poisoning", "acute mentalhealth illness", or "acute exacerbation of chronic mental-health illness". Data extracted: demographics, substances ingested, ED-disposition, hospital length of stay (LOS).

Results: Total ED presentations for older adults $\geq 60$ years in the sevenmonth periods: 2019: 30,189 and 2020: 26,450. Median age was 65 years. Older-DSP presentations: 2020: $n=37$ and 2019: $n=81$. This represented a significantly smaller proportion of $\geq 60$-year-old ED presentations in the 2020 period ( $0.14 \%$ vs. $0.3 \%$, OR: 0.5 , $95 \%$ CI: $0.3-0.8$, $p<0.001)$. There were more male gender total DSP and/or mental health presentations in 2020 than 2019 (49\% vs. $41 \% \%$, OR: 1.4, 95\% CI: $1-$ $1.9, p=0.05$ ). Similar proportions of DSP patients in both periods were treated solely in ED or had medical ward disposition (50\%). The proportion of presentations for isolated mental health-related problems was unchanged ( $n=263(0.9 \%), 2019$ and $n=236(0.9 \%), 2020)$. DSP-patient median ED and hospital-LOS were similar (2019: 12 hours (ED), sevendays (medical-ward) vs. 2020: 17 hours (ED), five-days (medical-ward)). Mental health admission median LOS was also similar (2019: 22 days vs. 2020: 24 days). The most common toxicants were sedative-hypnotics (2019: $32 \%$ vs. $2020: 29 \%$ ) and antidepressants (2019: $16 \%$ vs. 2020 : $18 \%)$.

Conclusion: This analysis demonstrated a significant decrease in older adults presenting to ED with DSP in the lockdown period of 2020 in Melbourne, Australia, and a notable increase in the proportion of males presenting with DSP and/or a mental health complaint.

\section{Abstract Withdrawn}

175. Expansion of Medical Toxicology Practice Through Telemedicine: an Example of Collaborative Antidote Administration

C E Hantsch ${ }^{1}$, A F Syed ${ }^{2,3}$

${ }^{1}$ Chicago, IL, USA. ${ }^{2}$ Loyola University Medical Center, Maywood, IL, USA. ${ }^{3}$ Loyola University Chicago Stritch School of Medicine, Maywood, IL, USA

Background: Medical toxicologists have expertise in management of poisoning. The American College of Medical Toxicology Directory lists only 56 current US inpatient practices. In 2020, the COVID-19 pandemic led to rapid expansion of telemedicine. Hypothesis: Telemedicine can expand inpatient medical toxicology practice through collaboration with in-person physicians.

Methods: This is a single-patient encounter case report. A 15-yearold female $(53.1 \mathrm{~kg})$ was admitted to the pediatric intensive care unit for management of diphenhydramine (1250-2500 mg) poisoning. Clinical findings included disorientation, hallucinations, agitation, hypertension $(151 / 87 \mathrm{mmHg})$, tachycardia (159 bpm), mydriasis, and dry mucous membranes. Standard drug screening detected benzodiazepines (pre-collection therapeutic use) but was otherwise negative. EKGs demonstrated transient QTc prolongation but no sodium channel blockade. The patient became increasingly agitated and combative despite a calm environment and lorazepam $(7 \mathrm{mg} / 12 \mathrm{~h})$. Existing telemedicine practice made available a HIPAA-compliant Microsoft Teams program for audio/visual connection using an iPad held by the patient's mother/staff. The remote toxicologist examined the patient and viewed the room. Through collaborative management, physostigmine rather than advanced sedation was utilized. The toxicologist, via telemedicine, provided dosing guidance and monitoring; the in-person intensivist monitored for adverse effects and was available for intervention.

Results: During antidote administration (two interval $0.5 \mathrm{mg}$ doses), the patient's agitation and disorientation resolved. Acute onset of sleepiness was attributed to benzodiazepine effect no longer countered by central anticholinergic toxicity. There were no adverse cardiovascular effects. The patient slept for several hours and had no further anticholinergic manifestations.
Conclusion: Telemedicine allowed for effective, real-time, collaborative patient care between a remote toxicologist and an in-person intensivist for an uncommon antidote administration. Both physicians were able to evaluate and monitor the patient from the perspective of their specialized expertise. Telemedicine for expansion of inpatient medical toxicology practice should be further explored.

\section{Residency Background of Medical Toxicology Fellows Within the Past 5 Years}

Louise W Kao

Indiana University, Indianapolis, IN, USA

Background: Non-Emergency Medicine (EM) applicants to Medical Toxicology (MT) fellowships have historically reported difficulty determining which programs would accept applications. In response, MT fellowship programs updated a document detailing applicant eligibility and the presence of a clinical requirement. To enhance the current document, we sought to add additional data regarding residency background of recent and current MT fellows.

Methods: All 27 program directors of ACGME accredited US MT fellowship programs were surveyed in March 2020 regarding the residency background of fellows within the past five years. Additionally, the ACMT fellowship database was queried regarding residency specialty of US MT fellows with graduation year 2015-2021.

Results: All MT fellowship program directors responded to the survey. Of 27 programs, $20(74 \%)$ accept applications outside of EM and 21 $(77.8 \%)$ have a clinical requirement. In the past five years, all $(100 \%)$ had fellows from categorical EM, nine (33.3\%) had Pediatrics, seven (25.9\%) had Internal Medicine, two (7.4\%) had Occupational/ Preventive Medicine, and three $(11.1 \%)$ had another specialty-Family Medicine and Forensic Medicine. Overall, 12 (37\%) had trained a fellow outside of categorical EM within the past five years, including seven (25.9\%) with a clinical requirement. For graduation year 2015-2021, there were 225 categorical EM fellows, five fellows with a combined EM background, and 21 non-EM fellows (8.4\%). The ACMT database does not verify completion of training.

Conclusion: MT fellowship training is available to applicants from all specialties, although currently most MT fellows are from EM. While there are likely several barriers for non-EM applicants, such as funding streams, we seek to inform incoming applicants regarding applicant eligibility at individual programs. It is encouraging that 12 of 27 programs, even those with a clinical requirement, have trained fellows outside of categorical EM in the past five years.

177. Consensus Development of a Core Content for a Standardized Medical Toxicology Curriculum for Medical Students

Charlotte E. Goldfine ${ }^{1}$, Derrick Lung ${ }^{2}$, Gillian Beauchamp ${ }^{3,4}$, Ayrn $\mathrm{O}^{\prime} \mathrm{Connor}^{5}$, Andrew Stolbach ${ }^{6}$, Louise Kao ${ }^{7}$, Bryan Judge ${ }^{8}$, Paul Wax ${ }^{9}$, Rahul Patwari ${ }^{10}$, Ziad Kazzi ${ }^{11}$

${ }^{1}$ Brigham and Women's Hospital, Boston, MA, USA. ${ }^{2}$ San Francisco General Hospital, San Francisco, CA, USA. ${ }^{3}$ Lehigh Valley Health Network, Allentown, PA, USA. ${ }^{4}$ University of South Florida Morsani College of Medicine, Allentown, PA, USA. ${ }^{5}$ Banner - University Medical Center Phoenix, Phoenix, AZ, USA. ${ }^{6}$ The Johns Hopkins Hospital, Baltimore, MD, USA. ${ }^{7}$ Indiana University School of Medicine, Indianapolis, IN, USA. ${ }^{8}$ Michigan State University College of Human Medicine, Grand Rapids, MI, USA. ${ }^{9}$ UT-Southwestern, Dallas, TX, USA. ${ }^{10}$ Rush Medical College, Chicago, IL, USA. ${ }^{11}$ Emory University Hospital, Atlanta, GA, USA

Background: Currently, no standardized core content in medical toxicology exists for medical students. Although there are several medical 
toxicology clerkships, little is known about their format and curricula. Therefore, the goals of this study were to (1) perform a survey of medical toxicology clerkship directors to assess the current state and needs of their clerkship and (2) develop a consensus-derived list of core topics that should be covered during a medical toxicology clerkship.

Methods: A cross-sectional survey was sent to all medical student clerkship directors in medical toxicology. A modified Delphi process was used by a task force established by the American College of Medical Toxicology (ACMT) to develop a set of core topics that should be covered during a medical student clerkship.

Results: The survey was completed by 19 out of 42 (45\%) clerkship directors. The majority of clerkships were four weeks in duration with a range of 1-40 students per year (average of 15 students/year). The three most common teaching methods used were bedside teaching $(94.44 \%, n$ $=17)$; classroom teaching $(94.44 \%, n=17)$; and journal club $(77.78 \%, n$ $=14)$. All of the clerkship directors $(100 \%, n=19)$ reported they would use a standardized curriculum and educational content developed by ACMT. Consensus was reached by the task force for a set of core topics that was differentiated into two tiers. Tier 1 topics (e.g., Pharmacology; Drugs; Drugs of Abuse; Natural Products; Pharmacological Basis of Antidote Use; Toxicologic Syndromes) were deemed essential for medical students, whereas tier 2 topics (e.g., Poison Centers; Laboratory and Other Diagnostic Assessments; Radiation Syndromes) were optional but strongly recommended.

Conclusions: This core content will serve as a framework for clerkship preceptors to provide medical students with a uniform exposure to medical toxicology. Additional work is needed to further develop a standardized curriculum and educational content for medical toxicology clerkships.

178. Interdisciplinary In-Situ Simulation of Drug Induced Hyperthermia in the Emergency Department Enhances Procedural Skills and Familiarity with Critical Equipment

Adam Blumenberg

Columbia University Medical Center, New York City, NY, USA

Background: Hyperthermic syndromes such as sympathomimetic toxicity may be rapidly lethal without immediate cooling. Intensive cooling strategies are rarely performed, and Emergency Department teams may be un-practiced in these techniques. Emergency department teams require skills training to achieve timely temperature management in an unstable hyperthermic patient.

Hypothesis: Interdisciplinary simulation in the clinical environment provides practice for rapid identification and cooling of hyperthermic patients.

Methods: This study analyzes an intra-department simulation curriculum and anonymized survey. A simulated case of agitated delirium with druginduced hyperthermia was presented in the resuscitation area of a large urban academic emergency department. A high-fidelity manikin as well as all of the material resources of the department were available. The case was administered by a medical toxicologist. The learners included five residents, five nurses, one pharmacist, one technician, three attendings, and two medical students. The case required learners to perform a primary survey, measure core temperature, locate rarely used equipment, and respond with advanced cooling techniques. A debrief was performed with discussion of the case and challenges of rapid cooling. An anonymous online survey was sent to all participants.

Results: During the case learners practiced with equipment such as ice, chilled fluid infusers, sedation and paralysis, cold water immersion, lavage equipment, and evaporative cooling devices. During the debrief, many learners verbally indicated practicing cooling techniques in the clinical setting improved their procedural skills and ability to locate and use critical equipment. Post hoc online survey universally indicated that learners valued the hands-on training. Likert scales indicated $100 \%$ agreement with the statement, "this experience will improve my performance in actual clinic setting." Multiple learners commented that practicing rapid cooling techniques enhanced their clinical skills.

Conclusion: High fidelity interdisciplinary in situ simulation of care for drug-induced hyperthermia provided practice locating and using equipment critical to rapidly cooling a patient.

\section{ACMT Annual Scientific Meeting Abstracts—Virtual}

\begin{tabular}{|c|c|}
\hline Author Name & Abstract No. \\
\hline Aaron, Cynthia & 146 \\
\hline Abesamis, Michael & $074,098,115$ \\
\hline Abia, Abia & 070 \\
\hline Acciani, Jennifer & 063 \\
\hline Ahmed, Zubair & 111 \\
\hline Al Falasi, Reem & 017 \\
\hline AL-Balushi, Hassan & 102 \\
\hline Al-Garadi, Mohammed & 011 \\
\hline Alaufi, Khalid & 150 \\
\hline Aldy, Kim & $016,052,160,161$ \\
\hline Allen, Lisa & 025,027 \\
\hline Aloumi, Abdullatif & 106,135 \\
\hline Alsarraf, Abdulaziz & 106 \\
\hline Altamimi, Abbas & 106 \\
\hline Alunnifegatelli, Danilo & 017 \\
\hline Amaducci, Alexandra & 038,047 \\
\hline Ames, Sarah & 169 \\
\hline Amine, Leena & 135 \\
\hline Anderson, Bruce & 020 \\
\hline Antoine, Jason & 109 \\
\hline Arens, Ann & 029,096 \\
\hline Aung, Arkar & 107 \\
\hline Aung, Ye & 107 \\
\hline Avera, Robert & 063 \\
\hline Babu, Kavita & 010 \\
\hline Baccanale, Cecile & 109 \\
\hline Barbuto, Alexander & 083,134 \\
\hline Barthol, Colleen & 060 \\
\hline Basse, Jack & 021 \\
\hline Bassett, Robert & 015 \\
\hline Bates, Breanna & 060 \\
\hline Baumgartner, Kevin & 021,064 \\
\hline Beam, Daren & 142 \\
\hline Beauchamp, Gillian & 038,177 \\
\hline Becker, Sarah & 066 \\
\hline Belcher, Rachel & 044 \\
\hline Benjamin, Denis & 137 \\
\hline Berg, Sarah & 008 \\
\hline Berk, Justin & 040 \\
\hline Berkowitz, Laureen & 040 \\
\hline Bhatia, Deven & 112 \\
\hline Blumenberg, Adam & 178 \\
\hline Borek, Heather & $028,095,169$ \\
\hline
\end{tabular}


Bourgeois, Florence

Brady, Mark

Brambl, Wells

Brandt, Rachel

Brant, Emily

Brenner, Marielle

Brent, Jeffrey

Bruccoleri, Rebecca

Buckley, Nicholas

Burns, Michele

Bush, Brian

Bux, Michael

Calleo, Vincent

Camarena-Michel, Alexa

Campleman, Sharan

Cannon, Robert

Cao, Dazhe James

Carpenter, Joseph

Carreiro, Stephanie

Castaneda, Jorge

Castaneda-Guarderas, Ana

Cavanaugh, Jared

Cavazzoni, Elena

Chandler, Beth

Chandran, Kira

Chapman, Brittany

Chary, Michael

Chen, Richard

Chesser, Taylor

Chhabra, Neeraj

Clark, Richard

Clark, Seth

Codding, Joshua

Cohen, Neta

Cole, Jon

Cook, Clayton

Cook, Madison

Cook, Matthew

Corbett, Bryan

Correia, Matthew

Cowan, Ethan

Crane, Christopher

Curry, Steven

D'Alessandro, Angelo

Danagoulian, Shooshan

Darracq, Michael

Dart, Richard

Davidson, Zachary

Davis, Adrienne

Davis-Martin, Rachael

Dawley, Diane

Dawson, Andrew
031

149

$006,114,145,155,170$

141

116

$063,069,073$

016, 021, 025

149,171

140

083, 084

144

101

068, 120, 139

056

021,159

038,047

$061,137,157$

130, 163

010, 080

111

149

055

165

152

012, 013, 039

010,080

084

105

149

091

053

040

058

082

029, 096, 113, 141

036

033

038

067, 072, 168

035, 054, 079

093

126

111

001

090

022, 023, 077

001, 122

094

082

010

036

140 de Castro Brás, Lisandra

de Olano, Jonathan

109

De Vries, Paige

DeBruyn, Brett

Demarco, Michael

Desale, Sameer

Di Nardo, Matteo

Dietzen, Dennis

Dolcourt, Bram

Dorey, Alyrene

Driver, Brian

Dugan, Christopher

Duns, Natalie

Eccleston, Lex

Ehlers, Paul

Eisenberg, Jonathan

Eisenstat, Matthew

Ekaney, Michael

Elbashir, Mohammed

Eldos, Yazeed

Ems, Raleigh

Epperson, Lindsey

Evankovich, John

Fikse, Derek

Fil, Laura

Filip, Ari

Filkins, Patrick

Finkelstein, Yaron

Fleeger, Tiffany

Fletcher, John

Foster, Sierra

Fox, Carolyn

Frey, Aaron

Froberg, Blake

Fullerton, Lynne

Galvis Blanco, Laura

Ganetsky, Michael

Gao, Han

Garlich, Fiona

Geib, Ann-Jeannette

Gerkin, Richard

Gerona, Roy

Girardi, Faye

Gittinger, Melissa

Goh, Chong Tien

Goldfine, Charlotte

Graudins, Andis

Greenblatt, Lawrence

Greene, Spencer

Gunn, Clare

Gutteridge, Daniel

Guzman-Soto, Andrés
102

029

118

166

012, 013, 039

017

034

086, 090

044, 103, 119, 127

029

140

165

109

009

066

055, 092, 130, 150

002

086

125,135

050

016

071

038, 047

058

008

118,163

082

081

060

052

002

095

142

128

082

156

060, 126

097, 148

005, 123

003

024

075

092, 118

165

156, 177

042, 046, 062, 131, 172, 173

036

021

005

044

102, 130, 163 


\begin{tabular}{|c|c|c|c|}
\hline Hack, Jason & 089 & Kim, Theresa & 018 \\
\hline Hack, Kaytlin & 159 & King, Andrew & $086,090,146$ \\
\hline Hafeez, Shaheyar & 060 & King, Joshua & 020 \\
\hline Hallett, Allen & 171 & Kirschner, Ron & 059 \\
\hline Hallett, Thao-Phuong & 171 & Klein-Schwartz, Wendy & 158 \\
\hline Halmo, Laurie & 032 & Kohman, Kelsey & 061 \\
\hline Hanson III, Hugo & 058 & Koons, Andrew & 038 \\
\hline Hantsch, C & 175 & Kopec, Kathryn & 005 \\
\hline Heard, Kennon & 001 & Korenoski, Amanda & 147 \\
\hline Heard, Susan & 001 & Kramer, Ryan & 036 \\
\hline Hendrickson, Robert & $075,079,132,153,154$ & Kreshak, Allyson & 164 \\
\hline Hernandez, Reynaldo & 015 & Krotulski, Alex & $025,027,092$ \\
\hline Herr, Daniel & 017 & Krueger, James & 105 \\
\hline Hiller, $\mathrm{H}$ & 134 & Kuan, Joel & 173 \\
\hline Hintze, Trager & 060 & LaBarge, Kara & 080 \\
\hline Hlaing, Tin & 107 & Lachman, Chaya & 020 \\
\hline Hodge, Kathie & 139 & Lasoff, Daniel & 085,100 \\
\hline Hodgman, Michael & 120 & Le, Nhunguyen & 097 \\
\hline Hoffman, Robert & $106,125,135$ & Leang, Yit Hung & 062 \\
\hline Hollingsworth, Allen & 091 & Leatherman-Arkus, Nohea & 157 \\
\hline Holstege, Christopher & $028,095,169$ & Lebin, Jacob & $009,030,037$ \\
\hline Horowitz, B Zane & $035,054,079,133,136,152,153$ & Lee, Hwee Min & 062 \\
\hline Hou, Helen & 119 & Lee, Stephen & 136 \\
\hline Hoyte, Christopher & 122 & Leikin, Jerrold & 018 \\
\hline Htun, Kyaw & 107,143 & Leonard, James & 020,158 \\
\hline Hughes, Adrienne & 132,153 & Lewis, Brian & 081,162 \\
\hline Hund, Jonathan & 120 & Liao, Lawrence & 057 \\
\hline Hurd, Yasmin & 027 & Liao, Lillian & 126 \\
\hline Iwanicki, Janetta & 032,110 & Lieu, Nathan & 165 \\
\hline Jacobs, Elizabeth & 086,146 & Lin, Amber & 075 \\
\hline Jason, Max & 138 & Liss, David & 034 \\
\hline Jona, Madeleine & 046 & Livshits, Zhanna & 078 \\
\hline Jones, Jeffrey & 057,081 & Lock, Ashley & 126 \\
\hline Jordison Keeler, Lee Ann & 004,040 & Lockman, Justin & 166 \\
\hline Judge, Bryan & $057,081,162,177$ & Logan, Barry & 025,027 \\
\hline Jungsuwadee, Paiboon & 076 & Loo, George & 093 \\
\hline Kaczor, Eric & 080 & Lopez, Annette & 079,136 \\
\hline Kagzi, Asim & 086 & Love, Jennifer & $011,160,161$ \\
\hline Kahane, Alyssa & 082 & Lung, Derrick & 177 \\
\hline Kaiser, Sasha & 110,122 & Lynch, Michael & $101,108,121,147$ \\
\hline Kao, Louise & 176,177 & Mainhart, Allison & 036 \\
\hline Karshenas, Dana & 159 & Makar, Gregory & 048 \\
\hline Kastner, Mark & 005 & Malley, Carin & $043,071,074,098,101,115,116$ \\
\hline Katz, Kenneth & 038,047 & Mangan, Kyle & 090 \\
\hline Kaur, Jasdip & 038 & Manini, Alex & 025,027 \\
\hline Kazzi, Ziad & $026,102,150,177$ & Marano, Marco & 017 \\
\hline Kelner, Rowan & 103 & Marino, Ryan & $007,050,104,108$ \\
\hline Kennedy, Joseph & 151 & Marraffa, Jeanna & 068,139 \\
\hline Kerns, William & 005 & Martel, Marc & 029 \\
\hline Kestenbom, Inbal & 082 & Mascarenas, Danielle & 128 \\
\hline Kiernan, Emily & 130,150 & Mathew, Mathew & 082 \\
\hline Kim, Hong & 017 & Mathews, Bonnie & 080 \\
\hline
\end{tabular}


Matsler, Nik

Mazer-Amirshahi, Maryann

McCracken, Dimity

McFalls, Joshua

McFarland, Ariana

McKenzie, Michelle

McKillop, Iain

McNulty, Richard

Meggs, William

Mete, Mihriye

Miano, Marlea

Micciche, Andrew

Michienzi, Avery

Miner, James

Minhaj, Faisal

Minns, Alicia

Misra, Satish

Monte, Andrew

Morgan, Brent

Moss, Michael

Mudan, Anita

Muir, Mark

Mullins, Michael

Mullins, Peter

Murphy IV, Charles

Murphy, Christine

Murphy, Lauren

Mycyk, Mark

Myint, Kyee

Naing, Myint

Nelson, Lewis

Nemanich, Antonia

Nguyen, Harrison

Nguyen, Michelle

Nogar, Joshua

Nogee, Daniel

Nolan, Joan

Nystrom, Paul

O'Connor, Ayrn

O'Rourke, Dorcas

Oakland, Carrie

Obilom, Cherie

Olives, Travis

Olmedo, Rubén

Olson, Karen

Ontiveros, Sam

Osterhoudt, Kevin

Overbeek, Daniel

Padilla, Gabriel

Palungwachira, Pakhawadee

Papsun, Donna

Parker Cote, Jennifer
056

012, 013, 019, 039, 159

140

016, 045, 061, 112

010

004

002

140,165

109

012, 013, 039

041

043, 071, 074, 098, 101, 115, 116

028

029

020,158

049, 053, 067, 100, 164

005

001

055

066

009, 030, 037

126

008, 021, 034, 064

019

037

002, 005

136

091,151

143

143

013, 014, 019, 039

018

092

096

$006,114,145,155,170$

150,163

166

096

177

109

113,141

016, 157

029, 094, 096, 141, 167

070

071

049, 053, 072, 100, 168

015, 166

084

003

026

092

036
Pasternak, Yehonatan

082

Paterson, Emily

158

Patwari, Rahul

177

Pavuluri, Suresh

051

Pepin, Lesley

Perrone, Jeanmarie

056

011,012

Phillips, Todd

045

Pilkerton, Rebecca

103

Pittman, Peggy

Pizon, Anthony

Porter, Lauren

Przybysz, Thomas

Ramdin, Christine

Rapier, Marie

Rapp Olsson, Malin

Rasmussen, Marcia

Reddy, Neha

Rege, Saumitra

Repplinger, Daniel

Reynolds, Kate

Richardson Jr, Scott

Richardson, Lynne

Rigney, Elaine

Riley, Brad

Rippee, Lindsey

Rittilert, Panee

Rockacy, Douglas

Rockhill, Karilynn

Rodd, Nicola

Rodriguez, Mayra

Roper, Stephen

Ross, Bryan

Ross, Jennifer

Routsolias, Joanne

Ruha, Anne-Michelle

Rumack, Barry

Rumantir, Maggie

Runyon, Michael

Rushton, William

Ryan, Erin

Sam, Khin

Samame, Valeria

Samuels, Elizabeth

Sarker, Abeed

Scanlon, Matthew

Schimmel, Jonathan

Schimpf, M

Schult, Rachel

Schwarz, Evan

Seifert, Steve

Seifi, Ali
109

116,159

041

123

014

044

032

059

004

028, 095, 169

009

001

036

027

088

057, 081

126

137

101

032

172

087, 099

034

142

169

091,151

003, 021

001

082

002

026,124

124

042

025

004, 040

011

007, 043, 071, 074, 098, 101, 104, 115,116

027,110

134

138

008, 021, 064

128

060 
Shah, Kartik

Shaker, Kerollos

Shankar, Nikash

Sharma, Kapil

Shastry, Siri

Shaw, Leslie

Shegog, Evan

Shively, Rachel

Shulman, Joshua

Sidlak, Alexander

Silver, Elizabeth

Singh, Brian

Sloan, Colgan

Sloan, Trey

Smith, Karen

Smolinske, Susan

Smollin, Craig

Soe, Kyaw

Sonn, Brandon

Soto, Pelayia

Spyres, Meghan

Srihari, Priya

Srisuma, Saphaphume

Stang, Jamie

Stoffel, Jaclyn

Stolbach, Andrew

Stork, Christine

Suen, Kyle

Sulaeman, Siba

Surmaitis, Ryan

Sweat, Christopher

Syed, A

Tarchick, John

Taylor, Todd

Tedford, Natalie

Temple, Courtney

Test, Gidon

Thaker, Ameet

Therriault, Colin

Thompson, John

Thompson, Michelle

Thompson, Trevonne

Thornton, Stephen

Toce, Michael

ToxIC Investigators Consortium (ToxIC)

Trautman, William

Trecki, Jordan

Tun, Zaw
123

$016,045,117$

012, 013, 039

112,117

093

166

093

051

007, 071, 074, 098, 115, 116

$007,104,108,121,147$

077

166

119

109

059

128

030, 037

143

001

019

003, 048, 111, 148

053,067

137

029

061

177

068

$102,150,163$

131

038

$113,141,167$

175

090

102

066

$079,132,133,152$

082

157

055, 092

$079,136,153,154$

048

144

022, 023, 077

031

003, 008, 016, 021 159, 160, 161, 162

098, 108

024

107,143
Uber, Julia

004

Ulici, Alexandru

113,167

Ulmer, T

134

Ushakumari, Deepu

123

Varelas, Lee

071

Varney, Shawn

060, 126

Verzwyvelt, Steven

109

Vidal, Kavey

025,027

Vidri, Ron

071

Vohra, Varun

086, 146

Wagner, Samuel

078

Wagstaff, Holden

127

Wahby, Krista

086

Walsh, Connor

163

Walter, Nicholas

017

Wang, George

032

Wang, Ralph

037

Wang, Tristan

139

Warpinski, George

065

Warrick, Brandon

128

Wax, Paul

016, 021, 025, 177

Weaver, Nicholas

066

Wei, Yudan

033

Wermuth, Mary

069, 073

Westover, Rachael

043, 071, 074, 098, 101, 115, 116

Whitworth, Brian

026

Widmer, Stephanie

$006,114,145,155,170$

Wiegand, Timothy

087, 088, 099, 138

004, 040

Wightman, Rachel

133

107,143

Win, Ko

$085,100,164$

Winkler, Garret

Wunsch, Caroline

040

Yang, Ouwen

101

Yang, Yuan-Chi

011

Yanta, Joseph

043

Yazdanyar, Ali

038

Yeh, Michael

026, 055, 092

Yourish, Harmony

071

Zaw, Thein

107,143

Zhang, Jian

033

Ziegler, Amy

121

Zuckerman, Matthew

056

Publisher's Note Springer Nature remains neutral with regard to jurisdictional claims in published maps and institutional affiliations. 